\title{
Developing Generic Prior Distributions for Common Cause Failure Alpha Factors and Causal Alpha Factors
}

Zhegang Ma

Corwin L. Atwood

John A. Schroeder

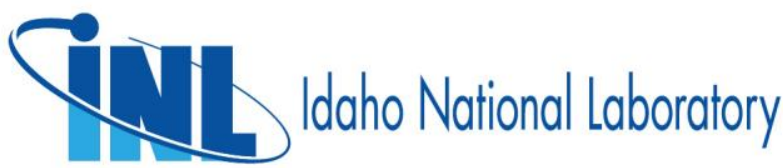




\section{DISCLAIMER}

This information was prepared as an account of work sponsored by an agency of the U.S. Government. Neither the U.S. Government nor any agency thereof, nor any of their employees, makes any warranty, expressed or implied, or assumes any legal liability or responsibility for the accuracy, completeness, or usefulness, of any information, apparatus, product, or process disclosed, or represents that its use would not infringe privately owned rights. References herein to any specific commercial product, process, or service by trade name, trade mark, manufacturer, or otherwise, does not necessarily constitute or imply its endorsement, recommendation, or favoring by the U.S. Government or any agency thereof. The views and opinions of authors expressed herein do not necessarily state or reflect those of the U.S. Government or any agency thereof. 
INL/EXT-21-43723

Revision 1 of INL/LTD-17-43723

\title{
Developing Generic Prior Distributions for Common Cause Failure Alpha Factors and Causal Alpha Factors
}

\author{
Zhegang $\mathrm{Ma}^{1}$ \\ Corwin L. Atwood ${ }^{2}$ \\ John A. Schroeder (Retired) ${ }^{1}$
}

August 2021

${ }^{1}$ Idaho National Laboratory Idaho Falls, Idaho 83415

2Statwood Consulting Silver Spring, MD 20910

Prepared for the

Division of Risk Analysis

Office of Nuclear Regulatory Research

U.S. Nuclear Regulatory Commission

NRC Agreement Number 31310019N0006

Task Order Number 31310019F0022 
Page intentionally left blank 


\begin{abstract}
This report is a revision of the original report, INL/LTD-17-43723.

Distribution of the original report was to the Nuclear Regulatory Commission (NRC) only, and the report was not made available to the public. The original report was revised as this report for public distribution.

This report presents the latest update of generic prior distributions for common cause failure (CCF) alpha factors, as well as the development of new generic prior distributions for CCF causal alpha factors. The history of CCF treatment and parameter estimations is reviewed. The existing process for developing generic prior distributions is reviewed and used to develop new priors for CCF alpha factors and causal alpha factors. For causal alpha factors, different priors are developed for the five different CCF cause groups: Component (GC), Design (GD), Environment (GE), Human (GH), and Other (GO). These generic prior distributions could be used in the Standardized Plant Analysis Risk (SPAR) models for CCF parameter estimation. The issues and preliminary thoughts regarding prior distribution development are documented. Potential future work is then proposed for improving the process of developing priors.
\end{abstract}


Page intentionally left blank 


\section{CONTENTS}

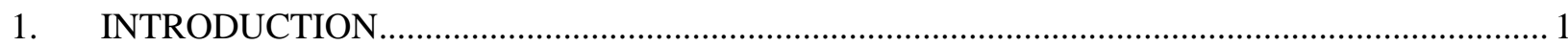

1.1 History of CCF Treatment and Parameter Estimations......................................................... 1

1.2 Prior Distributions in CCF Parameter Estimations .......................................................... 3

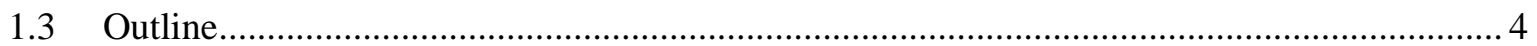

2. EXISTING PROCESS TO DEVELOP GENERIC PRIOR DISTRIBUTIONS ............................ 6

3. UPDATING GENERIC PRIOR DISTRIBUTIONS FOR ALPHA FACTORS ............................. 11

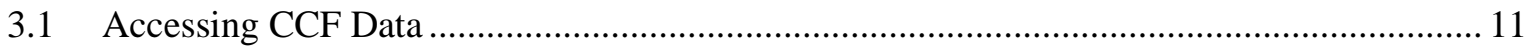

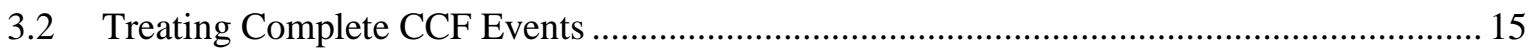

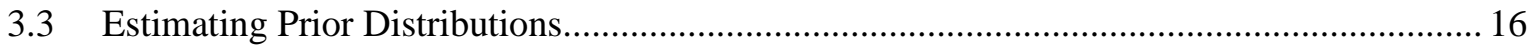

4. DEVELOPING GENERIC PRIOR DISTRIBUTIONS FOR CAUSAL ALPHA FACTORS ........ 21

4.1 Prior Distributions for the "Component" Cause Group (GC) ............................................. 21

4.2 Prior Distributions for the "Design" Cause Group (GD) ................................................ 27

4.3 Prior Distributions for the "Environment" Cause Group (GE) …........................................ 31

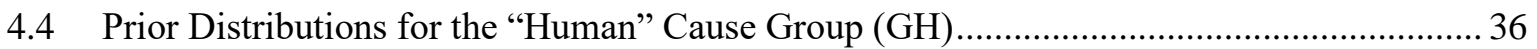

4.5 Prior Distributions for the "Other" Cause Group (GO) ....................................................... 40

5. VARIOUS ITEMS ON PRIOR DISTRIBUTION DEVELOPMENT AND CCF

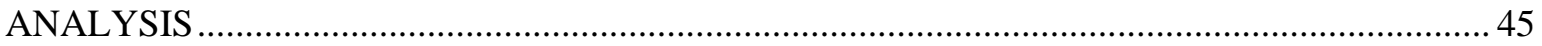

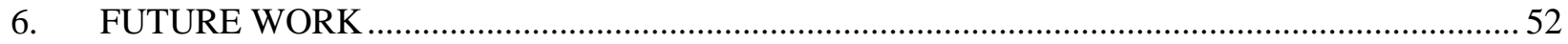

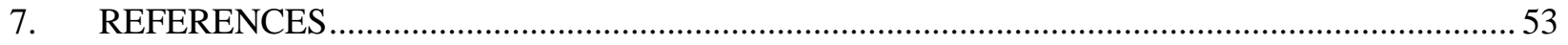

APPENDIX A PRIOR DISTRIBUTIONS IN PREVIOUS CCF PARAMETER ESTIMATION

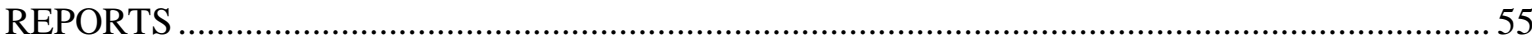

APPENDIX B EXAMPLE OF HOW TO PERFORM A BAYESIAN UPDATE ON CCF

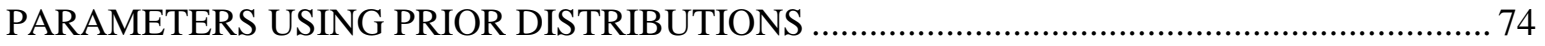

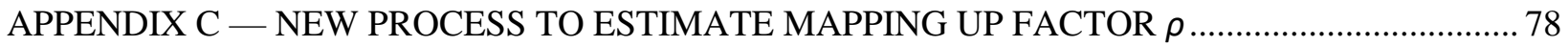

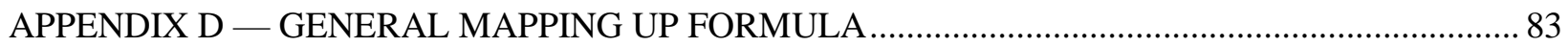




\section{FIGURES}

Figure 3-1. Selecting CCF event types and date ranges in the CCF Database. ....................................... 12

Figure 3-2. Defining CCF event characteristics in the CCF Database ....................................................... 13

Figure 3-3. CCF data, including CCF impact vectors that satisfy the selection criteria............................ 13

Figure 3-4. Homepage of the CalcPrior code for estimating alpha factor prior distributions..................... 18

Figure 3-5. Input to the CalcPrior code for estimating alpha factor prior distributions............................ 19

Figure 3-6. Prior distribution parameters calculated by the CalcPrior code........................................... 19

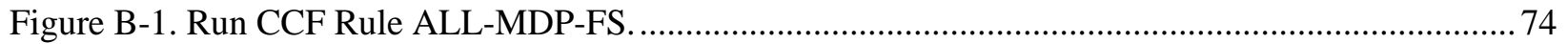

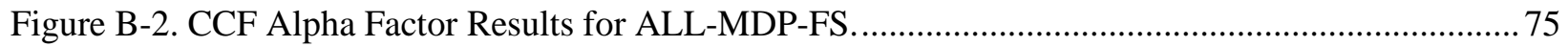

Figure B-3. ALL-MDP-FS CCF Alpha Factor Results for CCCG of 2 and 3........................................ 77

\section{TABLES}

Table 1. Date ranges of the data and some of mean alpha values for existing prior distributions............... 4

Table 2. CCF data (1995-2005) used in the 2010 white paper [18] .................................................... 7

Table 3. $n k$ values for the partial CCF events for years 1995-2005, as per the 2010 white paper

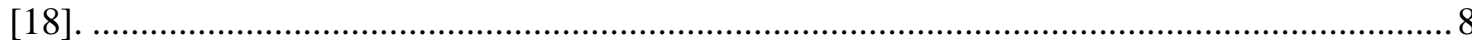

Table 4. Adjusted $n_{k}$ values for the prior distribution calculation, as per the 2010 white paper

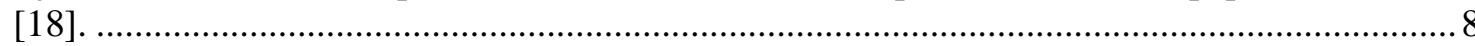

Table 5. Calculated alpha factor mean values in the 2010 white paper [18] ...................................... 9

Table 6. Estimated CCF industry-wide prior distributions in the 2010 white paper [18]........................ 10

Table 7. CCF events with unmapped impact vectors obtained from the CCF Database website............... 11

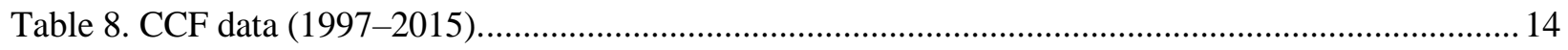

Table 9. $\mathrm{n}_{\mathrm{k}}$ values for the partial CCF events from 1997 through 2015. ............................................... 14

Table 10. CCF data (1997-2015) with curve-fitted complete CCF events............................................... 15

Table 11. Adjusted $n_{k}$ values for CCF events from 1997 through 2015 ............................................... 16

Table 12. Calculated alpha factor mean values for CCF events from 1997 through 2015 ....................... 17

Table 13. Estimated CCF industry-wide prior distributions with CCF events from 1997 through

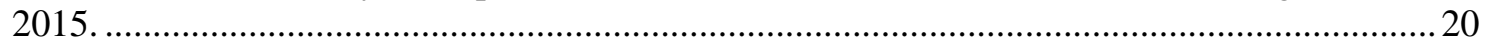

Table 14. CCF cause groups recommended in the alternative CCF model feasibility study....................22

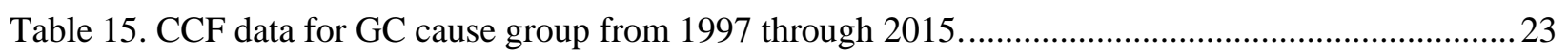

Table 16. nk values for the partial CCF events in the GC cause group from 1997 through 2015.............24

Table 17. Adjusted nk values for CCF events in the GC cause group from 1997 through 2015...............24

Table 18. Calculated alpha factor mean values for CCF events in the GC cause group from 1997 through 2015. 
Table 19. Estimated CCF industry-wide prior distributions with CCF events in the GC cause group from 1997 through 2015.

Table 20. CCF data for the GD cause group from 1997 through 2015 _.................................................2 27

Table 21. nk values for the partial CCF events in the GD cause group from 1997 through 2015.............28

Table 22. Adjusted nk values for CCF events in the GD cause group from 1997 through 2015...............28

Table 23. Calculated alpha factor mean values for CCF events in the GD cause group from 1997 through 2015

Table 24. Estimated CCF industry-wide prior distributions with CCF events in the GD cause

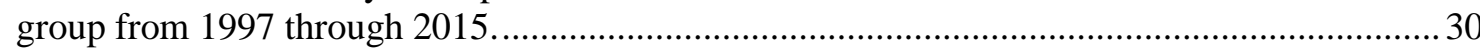

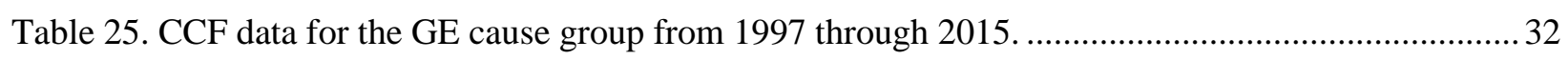

Table 26. nk values for the partial CCF events in the GE cause group from 1997 through 2015............. 32

Table 27. Adjusted nk values for CCF events in the GE cause group from 1997 through 2015............... 33

Table 28. Calculated alpha factor mean values for CCF events in the GE cause group from 1997 through 2015.

Table 29. Estimated CCF industry-wide prior distributions with CCF events in the GE cause

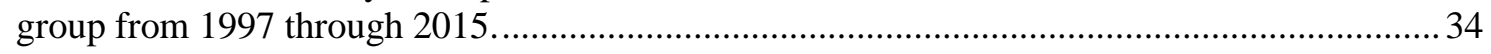

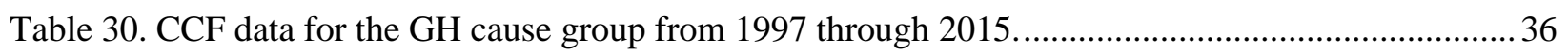

Table 31. nk values for the partial CCF events in the GH cause group from 1997 through 2015.............. 37

Table 32. Adjusted nk values for CCF events in the GH cause group from 1997 through 2015................37

Table 33. Calculated alpha factor mean values for CCF events in the GH cause group from 1997

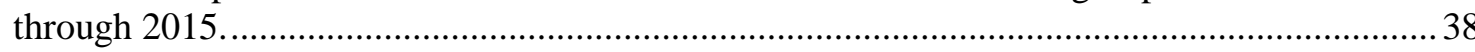

Table 34. Estimated CCF industry-wide prior distributions with CCF events in the GH cause

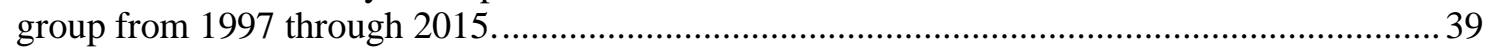

Table 35. CCF data for the GO cause group from 1997 through 2015 ............................................... 40

Table 36. nk values for the partial CCF events in the GO cause group from 1997 through 2015.............41

Table 37. Adjusted nk values for CCF events in the GO cause group from 1997 through 2015...............41

Table 38. Calculated alpha factor mean values for CCF events in the GO cause group from 1997 through 2015.

Table 39. Estimated CCF industry-wide prior distributions with CCF events in the GO cause group from 1997 through 2015

Table 40. Impact of mapping up factor. 


\section{ACRONYMS}

AFM alpha factor model

BFR binomial failure rate

BPM basic parameter model

CAFM causal alpha factor model

CCBE common cause basic event

CCCG common cause component group

$\mathrm{CC} \quad$ common cause

CCF common cause failure

ECA event and condition assessment

EDG emergency diesel generator

EPIX equipment performance and information exchange

FTR fail to run

FTS fail to start

INL Idaho National Laboratory

LER licensee event report

MLE maximum likelihood estimate

MM method of moments

NPRDS nuclear plant reliability data system

NRC Nuclear Regulatory Commission

NUREG Nuclear Regulatory Commission Regulation

PAFM partial alpha factor model

PRA probabilistic risk assessment

PWROG pressurized water reactor owner group

SAPHIRE Systems Analysis Programs for Hands on Integrated Reliability Evaluations

SCSS sequence coding and search system

SPAR Standardized Plant Analysis Risk

SQL structured query language

U.S. United States

WUG Westinghouse user group 


\section{Developing Generic Prior Distributions for Common Cause Failure Alpha Factors and Causal Alpha Factors}

\section{INTRODUCTION}

Common cause failures (CCFs) have been recognized as significant risk contributors, ever since the early launching of probabilistic risk assessments (PRAs) for commercial nuclear power plants (NPPs). Since the 1980s, a series of reports (e.g., those pertaining to U.S. Nuclear Regulatory Commission [NRC] regulations [NUREGs]) have been published to provide guidelines for performing CCF modeling using PRA and performing CCF event data analysis. A CCF database system was developed and is maintained by the NRC and Idaho National Laboratory (INL) for the U.S. commercial nuclear power industry. The CCF database system includes a CCF database that stores coded CCF events, and a CCF software that uses an impact vector and mapping method to estimate CCF parameters for the events stored in the CCF database. Generic prior distributions (or simply "prior distributions" or "priors") were developed and included in the CCF software for CCF Alpha Factor Model (AFM) parameter estimations. However, while the CCF database has been maintained ever since its development in late 1990s, and the CCF parameter estimations have been updated and published on a yearly basis, the process of developing prior distributions has not been published, and the prior distributions themselves have not been updated since the early 2000s. This report intends to uncover and review the existing process of developing prior distributions for CCF parameters, use recent data to update the prior distributions for CCF alpha factors, develop the prior distributions for causal alpha factors for use in the Causal Alpha Factor Model (CAFM), and document any issues and thoughts that arise regarding the CCF priors during this study.

This report is a revision of the original report, INL/LTD-17-43723. Distribution of the original report was to the NRC only, and the report was not made available to the public. The original report was revised as this report for public distribution.

\subsection{History of CCF Treatment and Parameter Estimations}

First, let us review the history of CCF treatment and parameter estimations in PRA. The following is a summary of the key reports on the development of CCF modeling guidelines and the NRC CCF database system.

NUREG/CR-4780 (also EPRI NP-5613), Procedures for Treating Common Cause Failures in Safety and Reliability Studies, Volumes 1 and 2 [1,2], was published in January 1988 to present the framework for including CCFs in risk and reliability evaluations. It provides procedures for performing and documenting CCF analysis via a practical, systematic approach. The framework includes the following four major stages: (1) system logic model development, (2) identification of common cause component groups, (3) common cause modeling and data analysis, and (4) system quantification and the interpretation of results. While it is not the purpose of the report "to advance or promote a particular method or technique," it does introduce the concept of impact vector for CCF event classification and representation, along with the mapping method that adjusts the original impact vectors to account for common cause group size differences in common cause parameter estimation. Appendix D of the report provides a detailed discussion on the background and justification of using the mapping method for parameter estimation. Although some doubts existed as regards the mapping method, especially the mapping up technique (when the component group size in the original system is smaller than in the system being analyzed) [3], use of the impact vector and mapping method was adopted in subsequent NRC CCF studies, becoming the state-of-the-practice in CCF parameter estimation.

NUREG/CR-6268, Common-Cause Failure Database and Analysis System (Volumes 1-4) [4,5,6,7], published in June 1998, extended previous CCF studies by introducing a method of collecting industry failure data, identifying and characterizing CCF events, and estimating CCF parameters and uncertainties using a computer software. The report relied on two data sources for CCF event identification: the 
Nuclear Plant Reliability Data System (NPRDS), which contains component failure information, and the Sequence Coding and Search System (SCSS), which contains Licensee Event Reports (LERs). Data from the years 1980-1995 were analyzed. The report describes a process by which analysts can consistently code CCF events. A CCF database system was developed, with a searchable CCF database for retrieving the CCF events of interest, and a CCF software for estimating CCF parameters. The CCF software stores CCF events and independent failure counts, and it estimates CCF parameters for the Alpha Factor and Multiple Greek Letter Models, based on the CCF event impact vector and mapping method.

NUREG/CR-5497, Common-Cause Failure Parameter Estimations [8], published in October 1998, documented the quantitative results of the CCF data collection effort described in Volumes 1-4 of NUREG/CR-6268 [4,5,6,7], as well as the insights from the CCF data analysis. It contains the CCF parameter estimates for most of the risk-important safety systems and components in commercial NPPs.

NUREG/CR-5485, Guidelines on Modeling Common-Cause Failures in Probabilistic Risk Assessment [9], published in November 1998, provided a set of guidelines to help PRA analysts model CCF events in commercial NPPs. The report combines the key aspects of the procedural guidelines presented in previous NRC CCF reports, provides additional insights from the CCF applications, and describes the CCF software capabilities and how to apply the CCF database information to PRA studies.

NUREG/CR-6268, Revision 1, Common-Cause Failure Database and Analysis System, Event Data Collection, Classification, and Coding [10], published in September 2007, updated the previous version's guidance on collecting, classifying, and coding CCF events. Three data sources are used for selecting equipment failure reports to be reviewed for CCF event identification: (1) the NPRDS, which contains component failure information from the years 1980-1996; (2) the Equipment Performance and Information Exchange (EPIX), which contains component failure information from the years since 1997; and (3) LER Search, which contains LERs. The updated CCF data analysis includes the following steps: collection of source data, identification of CCF events, coding of CCF events, database quality assurance, data analysis, and parameter estimation. The CCF event information and the independent event count are entered into the CCF database along with the quality assurance verification. The CCF software system uses the impact vector and mapping method to estimate CCF parameters. The impact vector method used in the process is based on the event's physical characteristics, including component degradation factor, timing factor, and shared cause factor. The software enables analysts to modify generic event impact factors for plant-specific applications, including using the mapping method to account for differences in common cause component group (CCCG) size.

A Series of NRC CCF Parameter Estimation Update Reports, published on the NRC website (http://nrcoe.inl.gov/ParamEstSpar/) starting in 2003, updated the CCF parameter estimations in NUREG/CR-5497 [8] on a yearly basis. Below is a list of these update reports, including the date range of the data used for the update.

CCF Parameter Estimation 2003 Update [11] reflects the version of the CCF database that contains data from 1980 to 2003. However, it uses a starting date of 1/1/1985 so as to avoid the large number of CCF events in the 1980-1984 period, as the trend decreases significantly between 1980 and 1985 . The analysis also found that the previously recommended maximum value of 0.85 for the mapping up factor, rho, was very conservative. A recommended maximum value of 0.50 for rho was used in the 2003 Update.

CCF Parameter Estimation 2005 Update [12] reflects the version of the CCF database that contains data from 1980 to 2005. It uses a starting date of 1/1/1991 so as to avoid the large number of CCF events in the 1980-1990 period, as the trend decreases significantly from 1980 to 1991.

CCF Parameter Estimation 2007 Update [13] reflects the version of the CCF database that contains data from 1980 to 2007. It uses a starting date of 1/1/1991 so as to avoid the large number of CCF events in the 1980-1990 period, as the trend decreases significantly from 1980 to 1991. 
CCF Parameter Estimation 2009 Update [14] reflects the version of the CCF database that contains data from 1997 to 2009. The starting date is 1/1/1997. The large number of CCF events in the 1980-1996 period are excluded from the analysis (and subsequent analyses), as the trend decreases significantly from 1980 to 1997.

CCF Parameter Estimation 2010 Update [15] reflects the version of the CCF database that contains data from 1997 to 2010. The starting date is 1/1/1997.

CCF Parameter Estimation 2012 Update [16] reflects the version of the CCF database that contains data from 1997 to 2012 . The starting date is $1 / 1 / 1997$.

CCF Parameter Estimation 2015 Update [17] reflects the CCF data contained within the CCF database by having executed the query rules in the folder SPAR Rules $2015 A$ on October 26,2016 . It contains data from 1/1/1997 to 12/31/2015.

It is worthwhile to note that, during the development and maintenance of the CCF database system, the whole process of data classification, loading, and parameter estimation underwent several levels of quality control. For example, all events are reviewed by two data analysts to ensure they are classified as CCF events and coded correctly. Then, a PRA analyst reviews the CCF events and results for consistency and compares them with PRA experience. A final review is performed by independent CCF experts (external to INL) who maintain the CCF database system for NRC. The independent review is usually conducted by CCF experts from industry organizations such as the Pressurized Water Reactor Owner Group (PWROG), formerly the Westinghouse User Group (WUG).

Nonetheless, CCF event identification and characterization remain subject to engineering judgement, as analysts could interpret the events in different ways and make various assumptions about the mission information, based on both the event reports and the physical and operational descriptions of the NPPs involved. The uncertainty caused by the data, as well as other uncertainties such as statistical uncertainty and modeling uncertainty, should be identified and properly addressed in CCF studies and applications.

\subsection{Prior Distributions in CCF Parameter Estimations}

NUREG/CR-5485 [9] discusses the data uncertainty inherent in developing a statistical database using CCF event reports. To develop an uncertainty distribution of CCF parameters, if one employs the Bayesian estimation procedure, the choice of prior distribution becomes critical. The prior distribution could reflect the analyst's subjective judgement or be based on observed ranges of variation in the parameters. Several different approaches are mentioned in NUREG/CR-5485:

1. Using the hierarchical Bayes method to develop a plant-to-plant variability distribution of various alpha factors (or other CCF model parameters) across all components and failure modes

2. Obtaining the maximum likelihood estimate (MLE) for a given alpha factor, then using a constrained noninformative prior as its uncertainty distribution in order to maximize the uncertainty given a constraint on the mean value; this distribution is usually broader than the corresponding hierarchical Bayes distribution

3. Using information from the constrained noninformative prior distributions to estimate the parameters of Dirichlet distributions for the CCCG. These estimates can be combined to obtain an effective estimate for the Dirichlet distribution parameter.

4. Using the mapping method to develop prior distributions for alpha factors pertaining to each CCCG size so as to utilize all CCF events in the CCF database. In this approach, all CCF events are mapped to a given CCCG size. The MLE for each alpha factor is obtained and fit using a constrained noninformative distribution. The estimates of the Dirichlet distribution parameters are calculated and combined to obtain an effective estimate. 
Using this final approach, NUREG/CR-5485 developed the prior distributions used for CCF parameter estimations in NUREG/CR-5497 [8]. However, the details of the process were not documented in the NUREG report or otherwise published. Instead, a white paper entitled "Estimation of IndustryWide Common-Cause Failure Prior Distributions" [18], dated January 2010, may be the best documentation so far that describes the process of estimating CCF generic prior distributions. This 2010 paper used the CCF data from the years 1995-2005 to develop the prior distributions and create step-bystep instructions.

Apart from the prior distribution results found in the 2010 paper [18] and in NUREG/CR-5485 [9], three other formal prior distributions are documented in the NRC CCF Parameter Estimation Update Reports: 2003 version, as per the 2003 update [11]; 2005 version, as per the 2005 update [12]; and 2007 version, as per the 2007 and subsequent updates [13-17]. Table 1 shows the date range of the data, along with the mean $\alpha$ values in each of these prior distributions. A copy of the prior distributions in NUREG/CR-5485 and the annual update reports is provided in Appendix A, while the prior distributions calculated in the 2010 paper is included in Section 2.

Table 1. Date ranges of the data and some of mean alpha values for existing prior distributions.

\begin{tabular}{|c|c|c|c|c|c|c|}
\hline Parameter & $\begin{array}{l}\text { NUREG/CR-5485 } \\
\text { NUREG/CR-5497 }\end{array}$ & $\begin{array}{l}2003 \text { CCF } \\
\text { Update }\end{array}$ & $\begin{array}{l}2005 \text { CCF } \\
\text { Update }\end{array}$ & $\begin{array}{l}2007 \text { CCF } \\
\text { Update }\end{array}$ & $\begin{array}{l}2010 \\
\text { Paper }\end{array}$ & $\begin{array}{c}\text { 2009/2010/2012/2015 } \\
\text { CCF Update }\end{array}$ \\
\hline $\begin{array}{l}\text { Date Range of } \\
\text { Failure Data }\end{array}$ & 1980-1995 & $\begin{array}{l}1985- \\
2003\end{array}$ & $\begin{array}{l}1991- \\
2005\end{array}$ & $\begin{array}{c}1991- \\
2007\end{array}$ & $\begin{array}{l}1995- \\
2005\end{array}$ & $\begin{array}{c}1997-2009 \\
/ 2010 / 2012 / 2015\end{array}$ \\
\hline $\begin{array}{c}\text { Version of } \\
\text { Priors in the } \\
\text { Report }\end{array}$ & $\begin{array}{c}\text { NUREG/CR-5485 } \\
\text { Version }\end{array}$ & $\begin{array}{c}2003 \\
\text { Version }\end{array}$ & $\begin{array}{c}2005 \\
\text { Version }\end{array}$ & $\begin{array}{c}2007 \\
\text { Version }\end{array}$ & $\begin{array}{c}2010 \\
\text { Version }\end{array}$ & 2007 Version \\
\hline$\alpha 2(\mathrm{CCCG}=2)$ & 4.70E-02 & 3.09E-02 & $4.06 \mathrm{E}-02$ & $2.57 \mathrm{E}-02$ & $1.75 \mathrm{E}-02$ & $2.57 \mathrm{E}-02$ \\
\hline$\alpha 3(\mathrm{CCCG}=3)$ & $2.58 \mathrm{E}-02$ & 7.17E-03 & 8.71E-03 & 5.79E-03 & $5.94 \mathrm{E}-03$ & 5.79E-03 \\
\hline$\alpha 4(\mathrm{CCCG}=4)$ & $1.86 \mathrm{E}-02$ & $3.72 \mathrm{E}-03$ & 4.64E-03 & $2.98 \mathrm{E}-03$ & $3.81 \mathrm{E}-03$ & $2.98 \mathrm{E}-03$ \\
\hline$\alpha 5(\mathrm{CCCG}=5)$ & $1.46 \mathrm{E}-02$ & $6.26 \mathrm{E}-04$ & 7.25E-04 & 5.33E-04 & $9.32 \mathrm{E}-04$ & $5.33 \mathrm{E}-04$ \\
\hline$\alpha 6(\mathrm{CCCG}=6)$ & $1.23 \mathrm{E}-02$ & $6.15 \mathrm{E}-04$ & $6.86 \mathrm{E}-04$ & 4.07E-04 & $5.06 \mathrm{E}-04$ & 4.07E-04 \\
\hline$\alpha 7(\mathrm{CCCG}=7)$ & $1.03 \mathrm{E}-02$ & $1.29 \mathrm{E}-04$ & $1.52 \mathrm{E}-04$ & $1.17 \mathrm{E}-04$ & 2.22E-04 & $1.17 \mathrm{E}-04$ \\
\hline$\alpha 8(\mathrm{CCCG}=8)$ & $9.06 \mathrm{E}-03$ & $1.38 \mathrm{E}-04$ & $1.46 \mathrm{E}-04$ & $1.25 \mathrm{E}-04$ & $1.88 \mathrm{E}-04$ & $1.25 \mathrm{E}-04$ \\
\hline $\begin{array}{l}\text { Version of } \\
\text { Priors Used in } \\
\text { Parameter } \\
\text { Estimate }\end{array}$ & Unclear & $\begin{array}{c}2003 \\
\text { Version }\end{array}$ & $\begin{array}{c}2005 \\
\text { Version }\end{array}$ & $\begin{array}{c}2005 \\
\text { Version }\end{array}$ & $\begin{array}{c}2010 \\
\text { Version }\end{array}$ & 2005 Version \\
\hline
\end{tabular}

On the other hand, the prior distributions used for CCF parameter estimates are embedded in the CCF software as a hardcopy table. They were compared with the 2003, 2005, and 2007 versions of the prior distributions, revealing that the software/database uses the 2005 version of prior distributions instead of the 2007 version. This means that although the 2007 version of prior distributions was published in the 2007 and subsequent updates [13-17], the 2005 version of prior distributions was actually used in those CCF parameter estimate updates.

\subsection{Outline}

The remainder of the report is organized as follows. Section 2 reviews the existing process of developing prior distributions, as described in the 2010 paper. Section 3 updates the prior distributions for alpha factors with data from the years 1997-2015. Section 4 develops prior distributions for causal alpha factors via a similar process and using the failure data from the years 1997-2015. Section 5 presents the 
issues encountered during the prior distribution development, as well as preliminary thoughts on these issues. Section 6 suggests potential future work for improving the CCF prior development process. Appendix A lists the CCF prior distributions as published in NUREG/CR-5485 and previous CCF parameter updates. Appendix B provides an example of how to perform a Bayesian update on CCF parameters using the prior distributions. Appendix $\mathrm{C}$ presents a new process that could be used to estimate the mapping up factor $\rho$. Appendix D provides explicit justification and an explicit general formula for the mapping up method in NUREG/CR-5485 [9]. 


\section{EXISTING PROCESS TO DEVELOP GENERIC PRIOR DISTRIBUTIONS}

To develop an uncertainty distribution of CCF parameters, one can employ the Bayesian estimation procedure, which makes the choice of prior distribution critical. The prior distribution could reflect the analyst's subjective judgement or be based on observed ranges of variation in the parameters. As discussed in Section 1.2, NUREG/CR-5485 [9] presents several methods to develop CCF prior distributions. The mapping method was used to develop prior distributions for alpha factors pertaining to each CCCG size, which utilizes all CCF events in the CCF database. In this method, all CCF events are mapped to a given CCCG size. The MLE for each alpha factor is obtained, then fit via a constrained noninformative distribution. The estimates of the Dirichlet distribution parameters for the CCCG are calculated and combined to obtain an effective estimate. However, the details of the process were not documented in NUREG/CR-5485 or other documents. Instead, a white paper titled "Estimation of Industry-Wide Common-Cause Failure Prior Distributions" [18], dated January 2010, may be the best documentation so far that describes the existing process of estimating CCF generic prior distributions. The 2010 white paper provides step-by-step instructions for developing a CCF prior distribution using an industry-wide dataset:

Step 1. For each CCCG size, tabulate the number of CCF events and complete CCF events. A complete $\mathrm{CCF}$ is defined as a CCF in which all redundant components are failed simultaneously as a direct result of a shared cause (i.e., the component degradation value equals 1.0 for all components and both the timing factor and the shared cause factor are equal to 1.0 .

Step 2. Calculate the $n_{k}$ 's for each group size (2-16), using all partial (i.e., incomplete) CCF events. This involves mapping up and mapping down. A partial CCF is a CCF with at least one of the CCF character parameters (component degradation value, timing factor, and shared cause factor) not being equal to 1.0).

Step 3. Using the information obtained in Step 1, perform a binomial regression to obtain the probability of CCF events in a given group size.

Step 4. Using the results from Step 3, obtain the estimated number of complete CCF events. Add this number to the final $n_{k}$ for each group size. For example, for group size 2 , add the number to $n_{2}$; for group size 4 , add it to $\mathrm{n}_{4}$.

Step 5. Using the final $n_{k}$ values, estimate the mean value alpha factors for each group size.

Step 6. Using these final $n_{i}$ values, estimate the beta prior distributions for each group size. The parameters of the beta distribution are $\alpha$ and $\beta$. The beta distribution is denoted by $\operatorname{Beta}(\alpha, \beta)$. A computer code, CalcPrior, was developed by INL to estimate the distributions via a procedure to calculate Dirichlet distribution parameters with noninformative prior distributions.

Step 7. As a check, calculate the mean of each prior distribution and compare them with the values obtained in Step 5. The mean value is obtained through the formula $\mu=\alpha /(\alpha+\beta)$.

The main difference between this process and the short descriptions in NUREG/CR-5485 seems to be that the process in the 2010 white paper separates the complete CCF events from the partial ones. While the impact vector and the mapping methods are used for partial CCF events, the binomial regression method is used to curve fit the complete CCF events. This is probably due to the concern that the mapping method might be adding too many pseudo-complete CCF events to other group sizes from the observed complete CCF events with the mapping method.

To explain the process, the 2010 white paper uses the CCF data from the years 1995-2005 as an example. This range was chosen because it was the most recent and reflected more current plant 
conditions and practices. For 1995-2005, there are 289 partial and 32 complete CCF events, with an average group size of 6.41. Table 2 shows the CCF data used in the white paper for Step 1.

Table 2. CCF data (1995-2005) used in the 2010 white paper [18].

\begin{tabular}{|c|c|c|c|c|c|}
\hline $\begin{array}{c}\text { Group } \\
\text { Size }\end{array}$ & $\begin{array}{c}\text { No. Partial } \\
\text { CCF Events }\end{array}$ & $\begin{array}{c}\text { No. Complete } \\
\text { CCF Events }\end{array}$ & $\begin{array}{c}\text { Total No. } \\
\text { CCF Events }\end{array}$ & $\begin{array}{c}\text { Probability of } \\
\text { Complete } \\
\text { CCF Event }\end{array}$ & $\begin{array}{c}\text { Estimated No. } \\
\text { Complete CCF } \\
\text { Events }\end{array}$ \\
\hline 2 & 55 & 25 & 80 & 0.22631 & 18.1048 \\
\hline 3 & 37 & 3 & 40 & 0.15199 & 6.0796 \\
\hline 4 & 57 & 2 & 59 & 0.09896 & 5.83864 \\
\hline 5 & 9 & 0 & 9 & 0.06305 & 0.56745 \\
\hline 6 & 15 & 0 & 15 & 0.0396 & 0.594 \\
\hline 7 & 3 & 0 & 3 & 0.02464 & 0.07392 \\
\hline 8 & 41 & 1 & 42 & 0.01525 & 0.6405 \\
\hline 9 & 3 & 0 & 3 & 0.0094 & 0.0282 \\
\hline 10 & 1 & 0 & 1 & 0.00578 & 0.00578 \\
\hline 11 & 9 & 0 & 9 & 0.00355 & 0.03195 \\
\hline 12 & 5 & 0 & 5 & 0.00218 & 0.0109 \\
\hline 13 & 4 & 0 & 4 & 0.00134 & 0.00536 \\
\hline 14 & 6 & 0 & 6 & 0.00082 & 0.00492 \\
\hline 15 & 1 & 0 & 1 & 0.0005 & 0.0005 \\
\hline 16 & 43 & 1 & 44 & 0.00031 & 0.01364 \\
\hline Total & 289 & 32 & 321 & & 32.00016 \\
\hline
\end{tabular}

In Step 2, the impact factors of 289 partial CCF events are mapped up or down to obtain the values of $n_{k}$ (the number of events involving failure of $\mathrm{k}$ similar components) for each group sized 2-16 (refer to [9] and [10] for the CCF event impact vector and mapping method). The number of independent events, $n_{I}$, for a given group size $\mathrm{m}$ is estimated via the following equation:

$$
n_{I}=\frac{N * m}{A V G}
$$

where $n_{I}=$ adjusted number of independent events for group size $\mathrm{m}$

$$
\begin{aligned}
& N=\text { total number of independent events } \\
& m=\text { group size }
\end{aligned}
$$$$
A V G=\text { average group size }
$$

Table 3 shows the $n_{k}$ values for the 289 partial CCF events obtained in the white paper.

In Step 3, the binomial regression method (see Ref. [19] and NUREG/CR-6823 [20]) rather than the mapping method is used to curve fit the fraction of complete CCF events over the total number of CCF events. Assuming the fraction of complete CCF events over the total number of CCF events to be $P$, the values in Table 2 (i.e., the columns for Group Size, No. Complete CCF Events, and Total No. CCF Events) are curve fitted via binomial regression. The results (i.e., Probability of Complete CCF Event and Estimated No. Complete CCF Events for each group size) are listed in the last two columns of Table 2.

In Step 4, Estimated No. Complete CCF Events in Table 2 is added to the final $n_{k}$ in Table 3 for each group size (e.g., $n_{2}$ for group size 2 and $n_{3}$ for group size 3 ). For example, in Table 2, the estimated number of complete CCF events for group size 2 is 18.1048 . This number is added to $n_{2}$ for group size 2 
in Table 2 (i.e., 19.7694) in order to obtain the adjusted $n_{2}$ value for group size 2 (i.e., 37.8742). The total number of failures, $n_{t}$, for each group size $m$ is also calculated via the following equation:

$$
n_{t}=n_{I}+\sum_{k=1}^{m} n_{k}
$$

Table 3. $n_{k}$ values for the partial CCF events for years 1995-2005, as per the 2010 white paper [18].

\begin{tabular}{|c|c|c|c|c|c|c|c|c|c|c|c|c|c|c|c|c|c|}
\hline $\begin{array}{c}\text { Group } \\
\text { Size } \\
\end{array}$ & $n_{I}$ & $\mathrm{n}_{1}$ & $\mathrm{n}_{2}$ & $\mathrm{n}_{3}$ & $\mathrm{n}_{4}$ & $\mathrm{n}_{5}$ & $\mathrm{n}_{6}$ & $\mathrm{n}_{7}$ & $\mathrm{n}_{8}$ & $\mathrm{n}_{9}$ & $\mathrm{n}_{10}$ & $\mathrm{n}_{11}$ & $\mathrm{n}_{12}$ & $\mathrm{n}_{13}$ & $\mathrm{n}_{14}$ & $\mathrm{n}_{15}$ & $\mathrm{n}_{16}$ \\
\hline 2 & 2023.0900 & 106.3113 & 19.7694 & & & & & & & & & & & & & & \\
\hline 3 & \begin{tabular}{|l}
1077.0168 \\
\end{tabular} & 116.5278 & 42.9392 & 13.0124 & & & & & & & & & & & & & \\
\hline 4 & 1436.0224 & 121.2871 & 55.2676 & 16.2645 & 10.3760 & & & & & & & & & & & & \\
\hline 5 & 1795.0280 & 131.1944 & 54.4966 & 25.7099 & 8.8633 & 4.3541 & & & & & & & & & & & \\
\hline 6 & 2154.0336 & 139.4990 & 54.8839 & 29.7565 & 14.9416 & 5.0185 & 2.6030 & & & & & & & & & & \\
\hline 7 & 2513.0391 & 145.8261 & 57.1983 & 30.9931 & 18.9744 & 9.3164 & 2.9235 & 1.5594 & & & & & & & & & \\
\hline 8 & 2872.0447 & 150.8235 & 60.1519 & 31.7431 & 20.8969 & 12.9359 & 5.9814 & 1.7075 & 0.9370 & & & & & & & & \\
\hline 9 & 3231.0503 & 156.5314 & 61.8792 & 32.0043 & 22.1217 & 15.2958 & 9.0293 & 3.8507 & 1.0019 & 0.5645 & & & & & & & \\
\hline 10 & 3590.0559 & 161.3800 & 64.1529 & 32.3025 & 22.6214 & 16.5976 & 11.4885 & 6.2910 & 2.4705 & 0.5913 & 0.3410 & & & & & & \\
\hline 11 & 3949.0615 & 165.4662 & 66.6130 & 33.3967 & 22.1117 & 17.3671 & 13.1500 & 8.5764 & 4.3481 & 1.5805 & 0.3519 & 0.2068 & & & & & \\
\hline 12 & 4308.0671 & 169.3230 & 68.5994 & 34.9270 & 21.7793 & 17.3869 & 14.1415 & 10.3999 & 6.3261 & 2.9791 & 1.0108 & 0.2117 & 0.1258 & & & & \\
\hline 13 & 4667.0727 & 172.8500 & 70.2772 & 36.7663 & 21.7364 & 17.0610 & 14.5053 & 11.6616 & 8.1210 & 4.6048 & 2.0271 & 0.6482 & 0.1289 & 0.0768 & & & \\
\hline 14 & 5026.0783 & 175.9892 & 71.8040 & 38.7455 & 22.0010 & 16.6733 & 14.4171 & 12.3592 & 9.5469 & 6.2428 & 3.3119 & 1.3742 & 0.4181 & 0.0796 & 0.0469 & & \\
\hline 15 & 5385.0839 & 178.8299 & 73.1061 & 40.8249 & 22.5243 & 16.3906 & 14.0766 & 12.5782 & 10.5183 & 7.7011 & 4.7246 & 2.3597 & 0.9311 & 0.2718 & 0.0498 & 0.0286 & \\
\hline 16 & 5744.0895 & 181.4401 & 74.1467 & 42.9721 & 23.2403 & 16.2910 & 13.6529 & 12.4509 & 11.0399 & 8.8463 & 6.1077 & 3.5267 & 1.6712 & 0.6326 & 0.1783 & 0.0316 & 0.0177 \\
\hline
\end{tabular}

Table 4 shows the adjusted $n_{k}$ values (including $n_{t}$ ) for group sizes $2-16$, and these can be used to calculate the parameter of the AFM or the industry-wide prior distribution mean values via the following MLE (Step 5):

$$
\begin{gathered}
\alpha_{1}=\frac{n_{I}+n_{1}}{n_{t}} \\
\alpha_{i}=\frac{n_{i}}{n_{t}}, \text { for } \mathrm{i}=2, \ldots, \mathrm{m}
\end{gathered}
$$

The values in Table 4 are used as input to CalcPrior, an INL-developed computer code, to estimate the industry-wide prior distributions with parameters $\alpha$ and $\beta$ (Step 6).

Table 5 and Table 6 show the results of the calculated industry-wide alpha factor mean values and prior distributions, respectively.

The 2010 white paper ends with the following caution regarding the use of industry-wide prior distributions: "A sufficient number of CCF and independent events is needed to obtain meaningful results when using the prior distributions. If sufficient events do not exist, then the data should not be binned so

\begin{tabular}{|c|c|c|c|c|c|c|c|c|c|c|c|c|c|c|c|c|c|c|}
\hline $\begin{array}{c}\text { Group } \\
\text { Size }\end{array}$ & $\mathrm{n}_{\mathrm{t}}$ & $\mathrm{n}_{\mathrm{I}}$ & $\mathrm{n}_{1}$ & $\mathrm{n}_{2}$ & $\mathrm{n}_{3}$ & $\mathrm{n}_{4}$ & $\mathrm{n}_{5}$ & $\mathrm{n}_{6}$ & $\mathrm{n}_{7}$ & $\mathrm{n}_{8}$ & $n_{9}$ & $\mathrm{n}_{10}$ & $\mathrm{n}_{11}$ & $\mathrm{n}_{12}$ & $\mathrm{n}_{13}$ & $\mathrm{n}_{14}$ & $\mathrm{n}_{15}$ & $\mathrm{n}_{16}$ \\
\hline 2 & 2167.2755 & 2023.09 & 106.3113 & 37.8742 & & & & & & & & & & & & & & \\
\hline 3 & 3213.1890 & 3034.63 & 116.5278 & 42.9392 & 19.0920 & & & & & & & & & & & & & \\
\hline 4 & 4255.2138 & 4046.18 & 121.2871 & 55.2676 & 16.2645 & 16.2146 & & & & & & & & & & & & \\
\hline 5 & 5282.9058 & 5057.72 & 131.1944 & 54.4966 & 25.7099 & 8.8633 & 4.9216 & & & & & & & & & & & \\
\hline 6 & 6316.5665 & 6069.27 & 139.4990 & 54.8839 & 29.7565 & 14.9416 & 5.0185 & 3.1970 & & & & & & & & & & \\
\hline 7 & 7347.6751 & 7080.81 & 145.8261 & 57.1983 & 30.9931 & 18.9744 & 9.3164 & 2.9235 & 1.6333 & & & & & & & & & \\
\hline 8 & 8378.1777 & 8092.36 & 150.8235 & 60.1519 & 31.7431 & 20.8969 & 12.9359 & 5.9814 & 1.7075 & 1.5775 & & & & & & & & \\
\hline 9 & 9406.2070 & 9103.9 & 156.5314 & 61.8792 & 32.0043 & 22.1217 & 15.2958 & 9.0293 & 3.8507 & 1.0019 & 0.5927 & & & & & & & \\
\hline 10 & 10433.6767 & 10115.44 & 161.3800 & 64.1529 & 32.3025 & 22.6214 & 16.5976 & 11.4885 & 6.2910 & 2.4705 & 0.5913 & 0.3410 & & & & & & \\
\hline 11 & 11460.1904 & 11126.99 & 165.4662 & 66.6130 & 33.3967 & 22.1117 & 17.3671 & 13.1500 & 8.5764 & 4.3481 & 1.5805 & 0.3519 & 0.2388 & & & & & \\
\hline 12 & 12485.7514 & 12138.53 & 169.3230 & 68.5994 & 34.9270 & 21.7793 & 17.3869 & 14.1415 & 10.3999 & 6.3261 & 2.9791 & 1.0108 & 0.2117 & 0.1367 & & & & \\
\hline
\end{tabular}
finely. Similar bins should be grouped based on engineering and environmental considerations."

Table 4. Adjusted $n_{k}$ values for the prior distribution calculation, as per the 2010 white paper [18]. 


\begin{tabular}{|c|c|c|c|c|c|c|c|c|c|c|c|c|c|c|c|c|c|c|}
\hline $\begin{array}{c}\text { Group } \\
\text { Size }\end{array}$ & $\mathrm{n}_{\mathrm{t}}$ & $\mathrm{n}_{\mathrm{I}}$ & $\mathrm{n}_{1}$ & $\mathrm{n}_{2}$ & $\mathrm{n}_{3}$ & $\mathrm{n}_{4}$ & $\mathrm{n}_{5}$ & $\mathrm{n}_{6}$ & $\mathrm{n}_{7}$ & $\mathrm{n}_{8}$ & $\mathrm{n}_{9}$ & $\mathrm{n}_{10}$ & $\mathrm{n}_{11}$ & $\mathrm{n}_{12}$ & $\mathrm{n}_{13}$ & $\mathrm{n}_{14}$ & $\mathrm{n}_{15}$ & $\mathrm{n}_{16}$ \\
\hline 13 & 13510.5500 & 13150.08 & 172.8500 & 70.2772 & 36.7663 & 21.7364 & 17.0610 & 14.5053 & 11.6616 & 8.1210 & 4.6048 & 2.0271 & 0.6482 & 0.1289 & 0.0822 & & & \\
\hline 14 & 14534.6346 & 14161.62 & 175.9892 & 71.8040 & 38.7455 & 22.0010 & 16.6733 & 14.4171 & 12.3592 & 9.5469 & 6.2428 & 3.3119 & 1.3742 & 0.4181 & 0.0796 & 0.0518 & & \\
\hline 15 & 15558.0861 & 15173.17 & 178.8299 & 73.1061 & 40.8249 & 22.5243 & 16.3906 & 14.0766 & 12.5782 & 10.5183 & 7.7011 & 4.7246 & 2.3597 & 0.9311 & 0.2718 & 0.0498 & 0.0291 & \\
\hline 16 & 16580.9696 & 16184.71 & 181.4401 & 74.1467 & 42.9721 & 23.2403 & 16.2910 & 13.6529 & 12.4509 & 11.0399 & 8.8463 & 6.1077 & 3.5267 & 1.6712 & 0.6326 & 0.1783 & 0.0316 & 0.0313 \\
\hline
\end{tabular}

Table 5. Calculated alpha factor mean values in the 2010 white paper [18].

\begin{tabular}{|c|c|c|c|c|c|c|c|c|}
\hline $\begin{array}{c}\text { Group } \\
\text { Size }\end{array}$ & $\alpha_{1}$ & $\alpha_{2}$ & $\alpha_{3}$ & $\alpha_{4}$ & $\alpha_{5}$ & $\alpha_{6}$ & $\alpha_{7}$ & $\alpha_{8}$ \\
\hline 2 & 0.982525 & $1.7475 \mathrm{E}-02$ & & & & & & \\
\hline 3 & 0.980694 & $1.3363 \mathrm{E}-02$ & $5.9417 \mathrm{E}-03$ & & & & & \\
\hline 4 & 0.979379 & $1.2988 \mathrm{E}-02$ & $3.8222 \mathrm{E}-03$ & $3.8106 \mathrm{E}-03$ & & & & \\
\hline 5 & 0.982209 & $1.0316 \mathrm{E}-02$ & $4.8666 \mathrm{E}-03$ & $1.6777 \mathrm{E}-03$ & $9.3161 \mathrm{E}-04$ & & & \\
\hline 6 & 0.982934 & $8.6891 \mathrm{E}-03$ & $4.7108 \mathrm{E}-03$ & $2.3656 \mathrm{E}-03$ & $7.9450 \mathrm{E}-04$ & $5.0614 \mathrm{E}-04$ & & \\
\hline 7 & 0.983527 & $7.7846 \mathrm{E}-03$ & $4.2179 \mathrm{E}-03$ & $2.5823 \mathrm{E}-03$ & $1.2679 \mathrm{E}-03$ & $3.9787 \mathrm{E}-04$ & $2.2229 \mathrm{E}-04$ & \\
\hline 8 & 0.983887 & $7.1796 \mathrm{E}-03$ & $3.7888 \mathrm{E}-03$ & $2.4943 \mathrm{E}-03$ & $1.5440 \mathrm{E}-03$ & $7.1394 \mathrm{E}-04$ & $2.0380 \mathrm{E}-04$ & $1.8829 \mathrm{E}-04$ \\
\hline 9 & 0.984502 & $6.5785 \mathrm{E}-03$ & $3.4025 \mathrm{E}-03$ & $2.3519 \mathrm{E}-03$ & $1.6262 \mathrm{E}-03$ & $9.5992 \mathrm{E}-04$ & $4.0938 \mathrm{E}-04$ & $1.0652 \mathrm{E}-04$ \\
\hline 10 & 0.984966 & $6.1487 \mathrm{E}-03$ & $3.0960 \mathrm{E}-03$ & $2.1681 \mathrm{E}-03$ & $1.5908 \mathrm{E}-03$ & 1.1011E-03 & $6.0296 \mathrm{E}-04$ & $2.3678 \mathrm{E}-04$ \\
\hline 11 & 0.985364 & $5.8125 \mathrm{E}-03$ & $2.9141 \mathrm{E}-03$ & $1.9295 \mathrm{E}-03$ & $1.5154 \mathrm{E}-03$ & 1.1474E-03 & $7.4836 \mathrm{E}-04$ & $3.7941 \mathrm{E}-04$ \\
\hline 12 & 0.985753 & $5.4944 \mathrm{E}-03$ & $2.7973 \mathrm{E}-03$ & $1.7444 \mathrm{E}-03$ & $1.3925 \mathrm{E}-03$ & $1.1326 \mathrm{E}-03$ & $8.3297 \mathrm{E}-04$ & $5.0667 \mathrm{E}-04$ \\
\hline 13 & 0.986113 & $5.2017 \mathrm{E}-03$ & $2.7212 \mathrm{E}-03$ & $1.6088 \mathrm{E}-03$ & $1.2628 \mathrm{E}-03$ & $1.0736 \mathrm{E}-03$ & $8.6317 \mathrm{E}-04$ & $6.0108 \mathrm{E}-04$ \\
\hline 14 & 0.986444 & $4.9401 \mathrm{E}-03$ & $2.6658 \mathrm{E}-03$ & $1.5136 \mathrm{E}-03$ & $1.1471 \mathrm{E}-03$ & $9.9192 \mathrm{E}-04$ & $8.5031 \mathrm{E}-04$ & $6.5686 \mathrm{E}-04$ \\
\hline 15 & 0.986754 & $4.6989 \mathrm{E}-03$ & $2.6241 \mathrm{E}-03$ & $1.4477 \mathrm{E}-03$ & $1.0535 \mathrm{E}-03$ & $9.0482 \mathrm{E}-04$ & $8.0849 \mathrm{E}-04$ & $6.7606 \mathrm{E}-04$ \\
\hline 16 & 0.987044 & $4.4720 \mathrm{E}-03$ & $2.5917 \mathrm{E}-03$ & $1.4016 \mathrm{E}-03$ & $9.8249 \mathrm{E}-04$ & $8.2340 \mathrm{E}-04$ & 7.5091E-04 & $6.6582 \mathrm{E}-04$ \\
\hline $\begin{array}{c}\text { Group } \\
\text { Size }\end{array}$ & $\alpha_{9}$ & $\alpha_{10}$ & $\alpha_{11}$ & $\alpha_{12}$ & $\alpha_{13}$ & $\alpha_{14}$ & $\alpha_{15}$ & $\alpha_{16}$ \\
\hline 9 & $6.3011 \mathrm{E}-05$ & & & & & & & \\
\hline 10 & $5.6672 \mathrm{E}-05$ & $3.2682 \mathrm{E}-05$ & & & & & & \\
\hline 11 & $1.3791 \mathrm{E}-04$ & $3.0706 \mathrm{E}-05$ & $2.0838 \mathrm{E}-05$ & & & & & \\
\hline 12 & $2.3861 \mathrm{E}-04$ & $8.0958 \mathrm{E}-05$ & $1.6955 \mathrm{E}-05$ & $1.0949 \mathrm{E}-05$ & & & & \\
\hline 13 & $3.4082 \mathrm{E}-04$ & $1.5003 \mathrm{E}-04$ & $4.7978 \mathrm{E}-05$ & $9.5404 \mathrm{E}-06$ & $6.0840 \mathrm{E}-06$ & & & \\
\hline 14 & $4.2951 \mathrm{E}-04$ & $2.2787 \mathrm{E}-04$ & $9.4548 \mathrm{E}-05$ & $2.8766 \mathrm{E}-05$ & $5.4767 \mathrm{E}-06$ & $3.5640 \mathrm{E}-06$ & & \\
\hline 15 & $4.9500 \mathrm{E}-04$ & $3.0368 \mathrm{E}-04$ & $1.5167 \mathrm{E}-04$ & $5.9844 \mathrm{E}-05$ & $1.7470 \mathrm{E}-05$ & $3.2009 \mathrm{E}-06$ & $1.8704 \mathrm{E}-06$ & \\
\hline 16 & $5.3351 \mathrm{E}-04$ & $3.6836 \mathrm{E}-04$ & $2.1269 \mathrm{E}-04$ & 1.0079E-04 & $3.8152 \mathrm{E}-05$ & $1.0753 \mathrm{E}-05$ & $1.9058 \mathrm{E}-06$ & $1.8877 \mathrm{E}-06$ \\
\hline
\end{tabular}


Table 6. Estimated CCF industry-wide prior distributions in the 2010 white paper [18].

\begin{tabular}{|c|c|c|c|c|c|c|c|c|c|c|c|c|c|c|c|c|}
\hline $\begin{array}{c}\text { Group } \\
\text { Size }\end{array}$ & $\alpha_{1}$ & $\mathrm{~b}_{1}$ & $\alpha_{2}$ & $b_{2}$ & $\alpha_{3}$ & $b_{3}$ & $\alpha_{4}$ & $b_{4}$ & $\alpha_{5}$ & $b_{5}$ & $\alpha_{6}$ & $\mathrm{~b}_{6}$ & $\alpha_{7}$ & $b_{7}$ & $\alpha_{8}$ & $\mathrm{~b}_{8}$ \\
\hline 2 & $2.661 \mathrm{E}+1$ & $4.732 \mathrm{E}-1$ & $4.732 \mathrm{E}-1$ & 2.661E+1 & & & & & & & & & & & & \\
\hline 3 & $5.341 \mathrm{E}+1$ & $1.051 \mathrm{E}+0$ & 7.278E-1 & $5.373 \mathrm{E}+1$ & 3.236E-1 & $5.414 \mathrm{E}+1$ & & & & & & & & & & \\
\hline 4 & $8.352 \mathrm{E}+1$ & $1.759 \mathrm{E}+0$ & $1.108 \mathrm{E}+0$ & $8.417 \mathrm{E}+1$ & 3.259E-1 & $8.495 \mathrm{E}+1$ & 3.250E-1 & $8.495 \mathrm{E}+1$ & & & & & & & & \\
\hline 5 & $1.628 \mathrm{E}+2$ & $2.949 \mathrm{E}+0$ & $1.710 \mathrm{E}+0$ & $1.640 \mathrm{E}+2$ & 8.065E-1 & $1.649 \mathrm{E}+2$ & $2.780 \mathrm{E}-1$ & $1.655 \mathrm{E}+2$ & $1.544 \mathrm{E}-1$ & $1.656 \mathrm{E}+2$ & & & & & & \\
\hline 6 & $2.338 \mathrm{E}+2$ & $4.060 \mathrm{E}+0$ & $2.067 \mathrm{E}+0$ & $2.358 \mathrm{E}+2$ & $1.121 \mathrm{E}+0$ & $2.368 \mathrm{E}+2$ & $5.628 \mathrm{E}-1$ & $2.373 \mathrm{E}+2$ & $1.890 \mathrm{E}-1$ & $2.377 \mathrm{E}+2$ & $1.204 \mathrm{E}-1$ & $2.378 \mathrm{E}+2$ & & & & \\
\hline 7 & $3.351 \mathrm{E}+2$ & $5.612 \mathrm{E}+0$ & $2.652 \mathrm{E}+0$ & $3.380 \mathrm{E}+2$ & $1.437 \mathrm{E}+0$ & $3.392 \mathrm{E}+2$ & \begin{tabular}{|l}
$8.797 \mathrm{E}-1$ \\
\end{tabular} & $3.398 \mathrm{E}+2$ & 4.319E-1 & 3.402E+2 & $1.355 \mathrm{E}-1$ & $3.405 \mathrm{E}+2$ & 7.573E-2 & $3.406 \mathrm{E}+2$ & & \\
\hline 8 & $4.202 \mathrm{E}+2$ & $6.882 \mathrm{E}+0$ & $3.066 \mathrm{E}+0$ & $4.240 \mathrm{E}+2$ & $1.618 \mathrm{E}+0$ & $4.255 \mathrm{E}+2$ & $1.065 \mathrm{E}+0$ & $4.260 \mathrm{E}+2$ & 6.595E-1 & $4.264 \mathrm{E}+2$ & 3.049E-1 & $4.268 \mathrm{E}+2$ & $8.704 \mathrm{E}-2$ & $4.270 \mathrm{E}+2$ & $8.042 \mathrm{E}-2$ & $4.270 \mathrm{E}+2$ \\
\hline 9 & $5.894 \mathrm{E}+2$ & $9.279 \mathrm{E}+0$ & $3.939 \mathrm{E}+0$ & $5.948 \mathrm{E}+2$ & $2.037 \mathrm{E}+0$ & $5.967 \mathrm{E}+2$ & $1.408 \mathrm{E}+0$ & $5.973 \mathrm{E}+2$ & $9.736 \mathrm{E}-1$ & $5.977 \mathrm{E}+2$ & 5.747E-1 & $5.981 \mathrm{E}+2$ & 2.451E-1 & $5.985 \mathrm{E}+2$ & $6.377 \mathrm{E}-2$ & $5.986 \mathrm{E}+2$ \\
\hline 10 & $7.606 \mathrm{E}+2$ & 1.161E+1 & $4.748 \mathrm{E}+0$ & 7.675E+2 & $2.391 \mathrm{E}+0$ & $7.698 \mathrm{E}+2$ & $1.674 \mathrm{E}+0$ & $7.705 \mathrm{E}+2$ & $1.228 \mathrm{E}+0$ & $7.710 \mathrm{E}+2$ & 8.503E-1 & $7.714 \mathrm{E}+2$ & 4.656E-1 & $7.717 \mathrm{E}+2$ & $1.828 \mathrm{E}-1$ & $7.720 \mathrm{E}+2$ \\
\hline 11 & 9.447E+2 & $1.403 \mathrm{E}+1$ & $5.573 \mathrm{E}+0$ & $9.532 \mathrm{E}+2$ & $2.794 \mathrm{E}+0$ & $9.560 \mathrm{E}+2$ & $1.850 \mathrm{E}+0$ & $9.569 \mathrm{E}+2$ & $1.453 \mathrm{E}+0$ & $9.573 \mathrm{E}+2$ & $1.100 \mathrm{E}+0$ & $9.576 \mathrm{E}+2$ & 7.175E-1 & $9.580 \mathrm{E}+2$ & $3.638 \mathrm{E}-1$ & $9.584 \mathrm{E}+2$ \\
\hline 12 & 1.181E+3 & $1.707 \mathrm{E}+1$ & $6.583 \mathrm{E}+0$ & $1.192 \mathrm{E}+3$ & $3.352 \mathrm{E}+0$ & $1.195 \mathrm{E}+3$ & $2.090 \mathrm{E}+0$ & $1.196 \mathrm{E}+3$ & $1.669 \mathrm{E}+0$ & $1.197 \mathrm{E}+3$ & $1.357 \mathrm{E}+0$ & $1.197 \mathrm{E}+3$ & 9.981E-1 & $1.197 \mathrm{E}+3$ & $6.071 \mathrm{E}-1$ & $1.198 \mathrm{E}+3$ \\
\hline 13 & $1.451 \mathrm{E}+3$ & $2.043 \mathrm{E}+1$ & $7.652 \mathrm{E}+0$ & 1.463E+3 & $4.003 \mathrm{E}+0$ & $1.467 \mathrm{E}+3$ & $2.367 \mathrm{E}+0$ & $1.469 \mathrm{E}+3$ & $1.858 \mathrm{E}+0$ & $1.469 \mathrm{E}+3$ & $1.579 \mathrm{E}+0$ & $1.469 \mathrm{E}+3$ & $1.270 \mathrm{E}+0$ & $1.470 \mathrm{E}+3$ & $8.842 \mathrm{E}-1$ & $1.470 \mathrm{E}+3$ \\
\hline 14 & $1.754 \mathrm{E}+3$ & $2.411 \mathrm{E}+1$ & $8.786 \mathrm{E}+0$ & $1.770 \mathrm{E}+3$ & $4.741 \mathrm{E}+0$ & $1.774 \mathrm{E}+3$ & 2.692E +0 & $1.776 \mathrm{E}+3$ & $2.040 \mathrm{E}+0$ & $1.776 \mathrm{E}+3$ & $1.764 \mathrm{E}+0$ & $1.777 \mathrm{E}+3$ & $1.512 \mathrm{E}+0$ & $1.777 \mathrm{E}+3$ & $1.168 \mathrm{E}+0$ & $1.777 \mathrm{E}+3$ \\
\hline 15 & $2.118 \mathrm{E}+3$ & $2.844 \mathrm{E}+1$ & $1.009 \mathrm{E}+1$ & 2.137E+3 & $5.634 \mathrm{E}+0$ & $2.141 \mathrm{E}+3$ & $3.108 \mathrm{E}+0$ & $2.144 \mathrm{E}+3$ & $2.262 \mathrm{E}+0$ & $2.145 \mathrm{E}+3$ & $1.943 \mathrm{E}+0$ & $2.145 \mathrm{E}+3$ & $1.736 \mathrm{E}+0$ & $2.145 \mathrm{E}+3$ & $1.451 \mathrm{E}+0$ & $2.145 \mathrm{E}+3$ \\
\hline 16 & $2.425 \mathrm{E}+3$ & 3.183E+1 & $1.099 \mathrm{E}+1$ & $2.446 \mathrm{E}+3$ & $6.368 \mathrm{E}+0$ & $2.451 \mathrm{E}+3$ & $3.444 \mathrm{E}+0$ & $2.454 \mathrm{E}+3$ & $2.414 \mathrm{E}+0$ & $2.455 \mathrm{E}+3$ & $2.023 \mathrm{E}+0$ & $2.455 \mathrm{E}+3$ & $1.845 \mathrm{E}+0$ & $2.455 \mathrm{E}+3$ & $1.636 \mathrm{E}+0$ & $2.456 \mathrm{E}+3$ \\
\hline $\begin{array}{c}\text { Group } \\
\text { Size }\end{array}$ & $\alpha_{9}$ & $\mathrm{~b}_{9}$ & $\alpha_{10}$ & $b_{10}$ & $\alpha_{11}$ & $b_{11}$ & $\alpha_{12}$ & $b_{12}$ & $\alpha_{13}$ & $b_{13}$ & $\alpha_{14}$ & $b_{14}$ & $\alpha_{15}$ & $b_{15}$ & $\alpha_{16}$ & $b_{16}$ \\
\hline 9 & $3.773 \mathrm{E}-2$ & $5.987 \mathrm{E}+2$ & & & & & & & & & & & & & & \\
\hline 10 & 4.376E-2 & $7.722 \mathrm{E}+2$ & $2.524 \mathrm{E}-2$ & $7.722 \mathrm{E}+2$ & & & & & & & & & & & & \\
\hline 11 & 1.322E-1 & $9.586 \mathrm{E}+2$ & 2.944E-2 & $9.587 \mathrm{E}+2$ & $1.998 \mathrm{E}-2$ & $9.587 \mathrm{E}+2$ & & & & & & & & & & \\
\hline 12 & $2.859 \mathrm{E}-1$ & $1.198 \mathrm{E}+3$ & $9.700 \mathrm{E}-2$ & $1.198 \mathrm{E}+3$ & 2.032E-2 & $1.198 \mathrm{E}+3$ & 1.312E-2 & $1.20 \mathrm{E}+3$ & & & & & & & & \\
\hline 13 & 5.014E-1 & $1.471 \mathrm{E}+3$ & $2.207 \mathrm{E}-1$ & 1.471E+3 & 7.057E-2 & $1.471 \mathrm{E}+3$ & 1.403E-2 & $1.47 \mathrm{E}+3$ & $8.95 \mathrm{E}-3$ & $1.47 \mathrm{E}+3$ & & & & & & \\
\hline 14 & 7.639E-1 & $1.778 \mathrm{E}+3$ & 4.052E-1 & $1.778 \mathrm{E}+3$ & $1.682 \mathrm{E}-1$ & $1.778 \mathrm{E}+3$ & 5.116E-2 & $1.78 \mathrm{E}+3$ & $9.74 \mathrm{E}-3$ & $1.78 \mathrm{E}+3$ & $6.34 \mathrm{E}-3$ & $1.78 \mathrm{E}+3$ & & & & \\
\hline 15 & $1.063 \mathrm{E}+0$ & $2.146 \mathrm{E}+3$ & $6.520 \mathrm{E}-1$ & $2.146 \mathrm{E}+3$ & $3.256 \mathrm{E}-1$ & $2.147 \mathrm{E}+3$ & 1.285E-1 & $2.15 \mathrm{E}+3$ & $3.75 \mathrm{E}-2$ & $2.15 \mathrm{E}+3$ & $6.87 \mathrm{E}-3$ & $2.15 \mathrm{E}+3$ & $4.02 \mathrm{E}-3$ & $2.15 \mathrm{E}+3$ & & \\
\hline 16 & $1.311 \mathrm{E}+0$ & $2.456 \mathrm{E}+3$ & 9.051E-1 & $2.456 \mathrm{E}+3$ & $5.226 \mathrm{E}-1$ & $2.457 \mathrm{E}+3 \mid$ & 2.477E-1 & $2.46 \mathrm{E}+3$ & $9.37 \mathrm{E}-2$ & $2.46 \mathrm{E}+3$ & $2.64 \mathrm{E}-2$ & $2.46 \mathrm{E}+3$ & $4.68 \mathrm{E}-3$ & $2.46 \mathrm{E}+3$ & $4.64 \mathrm{E}-3$ & $2.46 \mathrm{E}+3$ \\
\hline
\end{tabular}




\section{UPDATING GENERIC PRIOR DISTRIBUTIONS FOR ALPHA FACTORS}

This section updates the generic prior distributions for alpha factors, using CCF data for the years 1997-2015 in addition to the existing process described in Section 2, with changes applied as deemed necessary. The 1997-2015 period was the most recent date range of the CCF data available when the analysis was performed for the original report INL/LTD-17-43723, reflecting more current plant conditions and practices at the time. Furthermore, 1997 is the earliest year selectable on the CCF Database website.

\subsection{Accessing CCF Data}

CCF data stored in the NRC CCF Database system (https://rads.inl.gov/Pages/CCF.aspx) can be accessed and used for CCF analysis. ${ }^{a}$ The CCF Database website includes various CCF rules for selecting the CCF date range and other CCF event characteristics, such as component types of interest, failure modes, and failure causes. Through the CCF Database website, CCF events of interest can be obtained by selecting the proper CCF event characteristics. It can generate both the number of CCF events and the effective independent event count that satisfy the selection criteria. In addition, the CCF Database website can provide the original (or unmapped) impact vector for each selected CCF event, the mapped impact vector, and adjusted independent counts for different group sizes. The impact vector results can be output for further analysis. Table 7 shows examples of CCF events whose unmapped impact vectors were obtained from the CCF Database website.

Table 7. CCF events with unmapped impact vectors obtained from the CCF Database website.

\begin{tabular}{|c|c|c|c|c|c|c|c|c|c|c|c|c|c|c|c|c|}
\hline CCF Event & 1 & 2 & 3 & 4 & 5 & 6 & 7 & 8 & 9 & 10 & 11 & 12 & 13 & 14 & 15 & 16 \\
\hline $219-1997-0278$ & 0 & 1 & 0 & 0 & -- & -- & -- & -- & -- & -- & - & -- & -- & -- & -- & -- \\
\hline $219-1998-0207$ & 1.8 & 0.1 & -- & -- & -- & -- & -- & -- & -- & -- & -- & -- & -- & -- & -- & -- \\
\hline $219-1999-0248$ & 0 & 1 & 0 & 0 & -- & -- & -- & -- & -- & -- & -- & -- & -- & -- & -- & -- \\
\hline $219-2000-0051$ & 0 & 1 & 0 & 0 & 0 & 0 & 0 & 0 & -- & -- & -- & -- & -- & -- & -- & -- \\
\hline $219-2003-0369$ & 0 & 0 & 1 & 0 & 0 & 0 & -- & -- & -- & -- & -- & -- & -- & -- & -- & -- \\
\hline $219-2005-0341$ & 0 & 0 & 1 & 0 & 0 & 0 & -- & -- & -- & -- & -- & -- & -- & -- & -- & -- \\
\hline $219-2014-0488$ & 0 & 1 & 0 & 0 & 0 & -- & -- & -- & -- & -- & -- & -- & -- & -- & -- & -- \\
\hline $220-2001-0398$ & 0 & 0 & 0 & 1 & -- & -- & -- & -- & -- & -- & -- & -- & -- & -- & -- & -- \\
\hline $220-2007-0144$ & 0 & 0 & 0 & 1 & 0 & 0 & 0 & 0 & -- & -- & -- & -- & -- & -- & -- & -- \\
\hline $220-2010-0412$ & 0 & 1 & 0 & 0 & -- & -- & -- & -- & -- & -- & -- & -- & -- & -- & -- & -- \\
\hline $237-1998-0219$ & 1.45 & 0.025 & -- & -- & -- & -- & -- & -- & -- & -- & -- & -- & -- & -- & -- & -- \\
\hline $237-2004-0336$ & 0 & 1 & 0 & 0 & 0 & 0 & -- & -- & -- & -- & -- & -- & -- & -- & -- & -- \\
\hline
\end{tabular}

CCF data from 1997-2015 were chosen for this study, as this period represents more recent plant conditions and practices that were in place when the analysis was performed. On the CCF Database website, the following selection criteria are defined:

- Type of CCF Event Level: All Level CCF Events

- CCF Event Type: CCF Events Only

- Date Range: 1997-2015

a The NRC CCF Database system includes proprietary information and is not available to the public. 
- Filter Independent Events by Selected Cause(s): True

- Shock Criteria: All Events

- Redundancy Range: Minimum = 2, Maximum = 16

- Bayesian Update Method: Mean Method

- Failure Modes: select all failure modes except Setpoint

- Plants/Systems/Components/CCF Categories: No Selection on These CCF Event Characteristics

A total of $268 \mathrm{CCF}$ events and 7,492.8 effective independent failure events correspond to the above selection criteria. Figure 3-1, Figure 3-2, and Figure 3-3 show screenshots of the CCF Database website to illustrate the CCF selection criteria and results.

Additional criterion on CCF Categories $\rightarrow$ Degree $\rightarrow$ Almost/Partial or Complete is used to obtain the partial CCF events and complete CCF events, as required in the existing process. The unmapped/mapped impact vectors are also acquired from the CCF Database website. The mapped impact vectors for partial CCF events for each group size, as obtained from the website, are used directly in this study.

Table 8 shows the number of partial CCF events, the number of complete CCF events, and the total number of $\mathrm{CCF}$ events. Table 9 shows the mapped impact vectors for partial CCF events pertaining to each group size (2-16), as obtained from the CCF Database website.

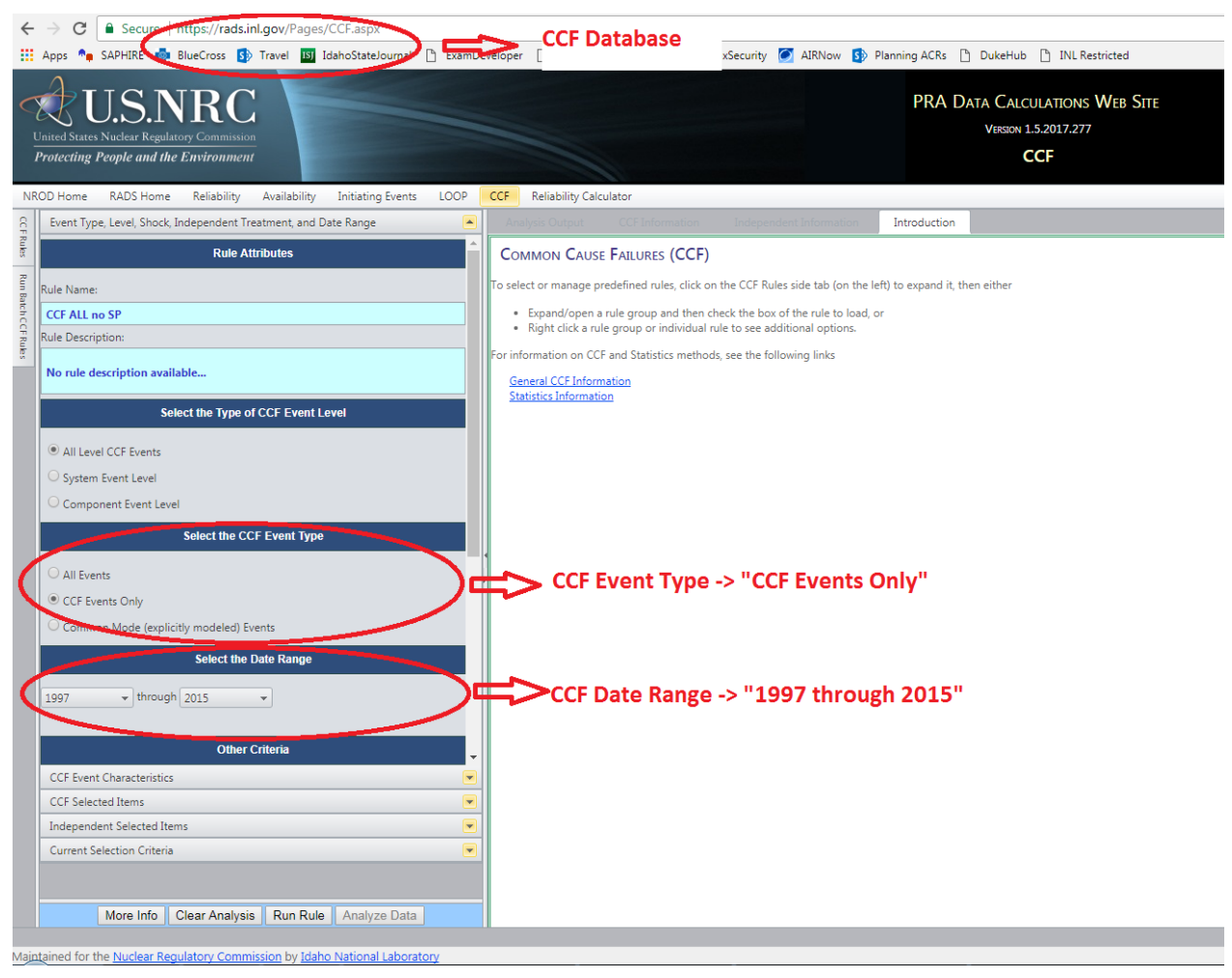

Figure 3-1. Selecting CCF event types and date ranges in the CCF Database. 


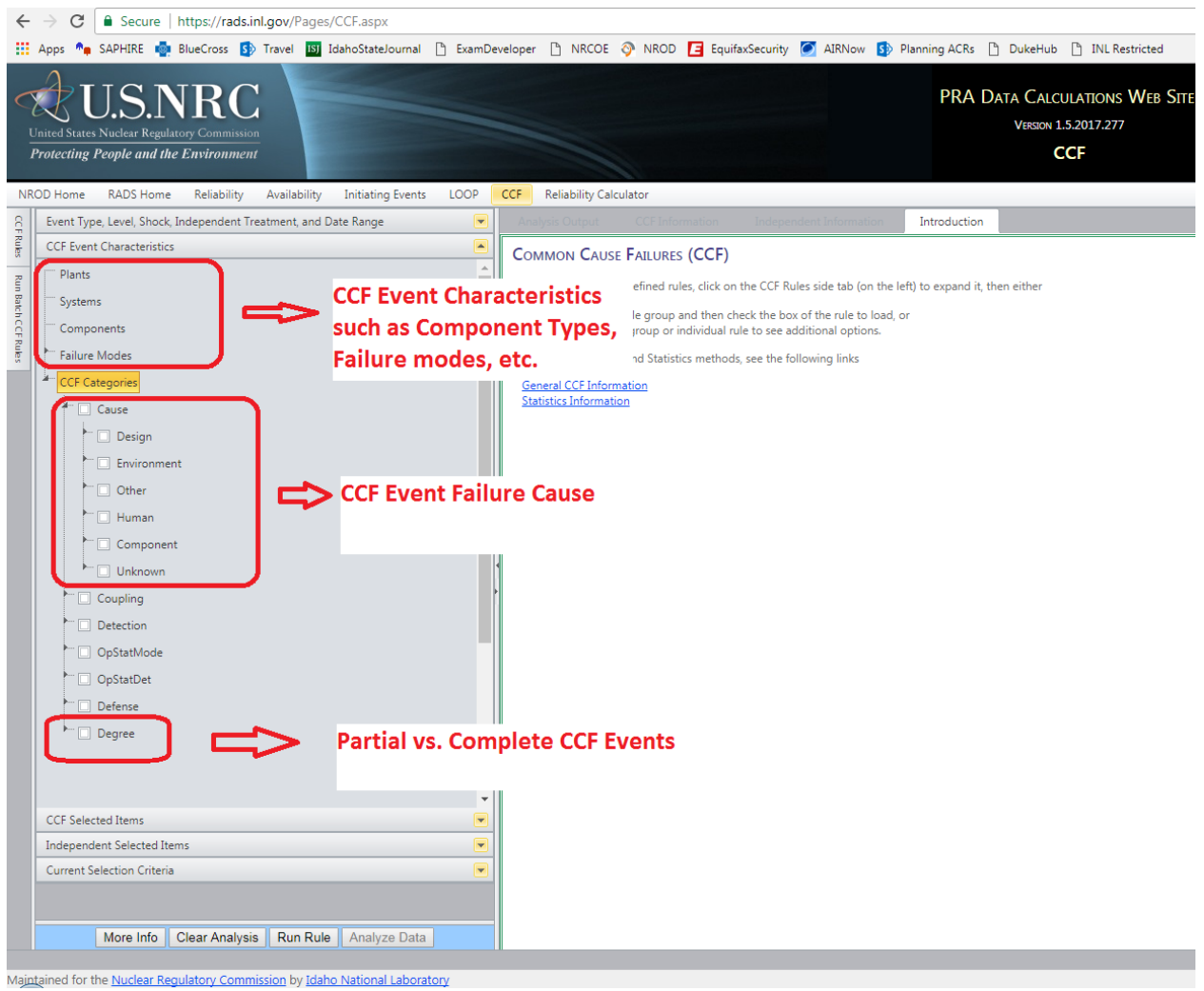

Figure 3-2. Defining CCF event characteristics in the CCF Database.

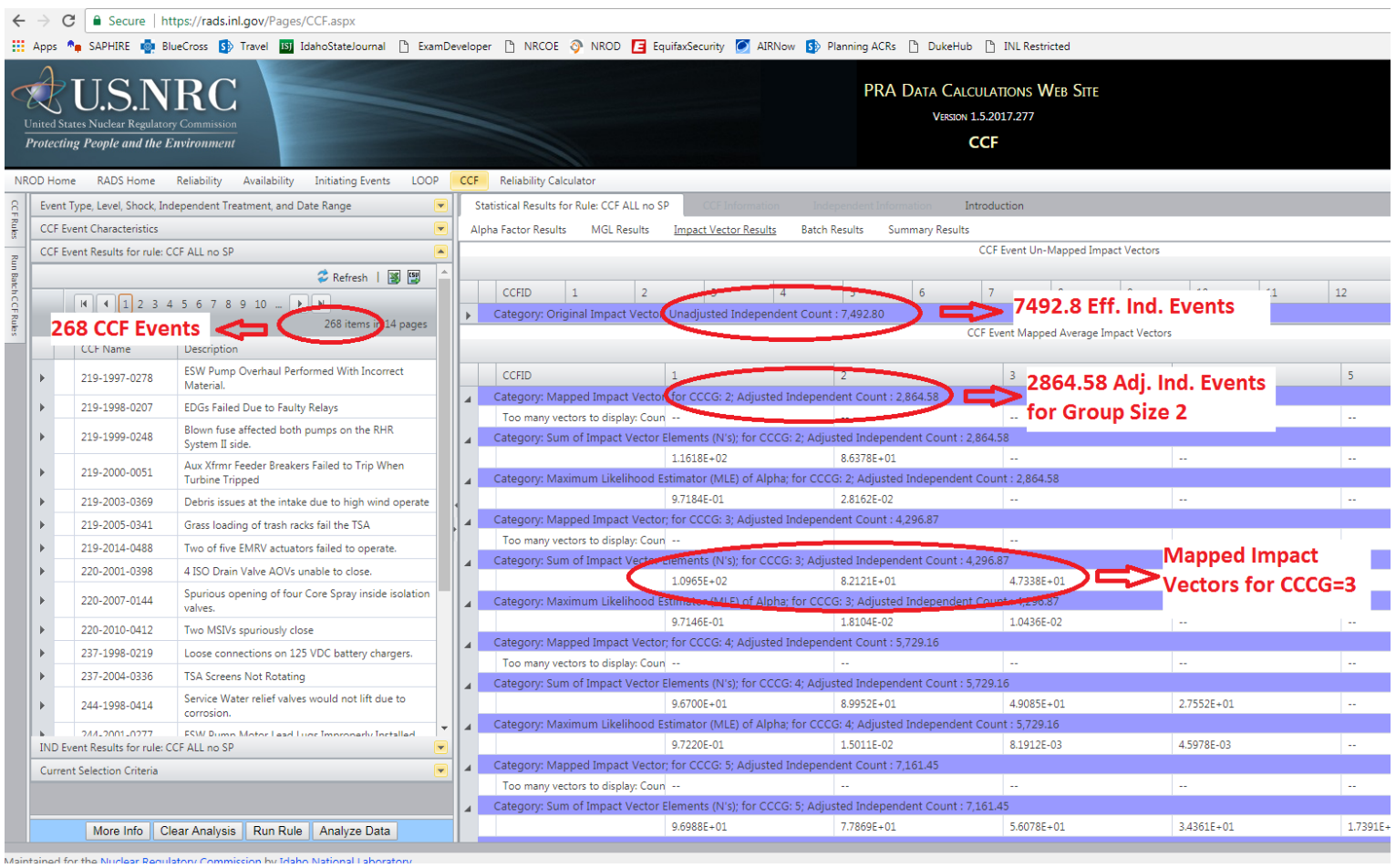

Figure 3-3. CCF data, including CCF impact vectors that satisfy the selection criteria. 
Table 8. CCF data (1997-2015).

\begin{tabular}{|c|c|c|c|}
\hline Group Size & $\begin{array}{c}\text { No. Partial } \\
\text { CCF Events }\end{array}$ & $\begin{array}{c}\text { No. Complete } \\
\text { CCF Events }\end{array}$ & $\begin{array}{c}\text { Total No. } \\
\text { CCF Events }\end{array}$ \\
\hline 2 & 27 & 34 & 61 \\
\hline 3 & 27 & 12 & 39 \\
\hline 4 & 61 & 2 & 63 \\
\hline 5 & 7 & 0 & 7 \\
\hline 6 & 30 & 5 & 35 \\
\hline 7 & 3 & 0 & 3 \\
\hline 8 & 30 & 2 & 32 \\
\hline 9 & 0 & 0 & 0 \\
\hline 10 & 0 & 0 & 0 \\
\hline 11 & 5 & 0 & 5 \\
\hline 12 & 7 & 1 & 8 \\
\hline 13 & 0 & 0 & 0 \\
\hline 14 & 1 & 0 & 1 \\
\hline 15 & 0 & 0 & 0 \\
\hline 16 & 14 & 0 & 14 \\
\hline Total & 212 & 56 & 268 \\
\hline
\end{tabular}

Table 9. $n_{k}$ values for the partial CCF events from 1997 through 2015.

\begin{tabular}{|c|c|c|c|c|c|c|c|c|c|c|c|c|c|c|c|c|}
\hline $\begin{array}{c}\text { Group } \\
\text { Size }\end{array}$ & $\mathrm{n}_{1}$ & $\mathrm{n}_{2}$ & $\mathrm{n}_{3}$ & $\mathrm{n}_{4}$ & n5 & n6 & $\mathrm{n} 7$ & $\mathrm{n} 8$ & n9 & $\mathrm{n}_{10}$ & $\mathrm{n}_{11}$ & $\mathrm{n}_{12}$ & $\mathrm{n}_{13}$ & $\mathrm{n}_{14}$ & $\mathrm{n}_{15}$ & $\mathrm{n}_{16}$ \\
\hline 2 & 115.61 & 31.164 & & & & & & & & & & & & & & \\
\hline 3 & 109.23 & 64.693 & 9.267 & & & & & & & & & & & & & \\
\hline 4 & 96.47 & 80.938 & 25.857 & 4.038 & & & & & & & & & & & & \\
\hline 5 & 96.92 & 73.190 & 39.900 & 14.539 & 2.141 & & & & & & & & & & & \\
\hline 6 & 98.05 & 65.654 & 46.584 & 23.001 & 8.533 & 1.209 & & & & & & & & & & \\
\hline 7 & 101.15 & 61.106 & 44.388 & 29.499 & 15.401 & 5.219 & 0.716 & & & & & & & & & \\
\hline 8 & 102.97 & 59.825 & 41.221 & 31.486 & 20.828 & 10.368 & 3.197 & 0.453 & & & & & & & & \\
\hline 9 & 104.47 & 59.373 & 38.811 & 31.849 & 23.262 & 14.602 & 7.206 & 2.091 & 0.298 & & & & & & & \\
\hline 10 & 105.31 & 59.671 & 37.235 & 31.000 & 24.661 & 17.351 & 10.623 & 5.043 & 1.393 & 0.2028 & & & & & & \\
\hline 11 & 105.59 & 60.457 & 36.289 & 29.832 & 25.016 & 19.214 & 13.174 & 7.876 & 3.546 & 0.9485 & 0.1428 & & & & & \\
\hline 12 & 105.79 & 61.357 & 35.385 & 29.051 & 24.559 & 20.390 & 15.020 & 10.190 & 5.885 & 2.5054 & 0.6629 & 0.1033 & & & & \\
\hline 13 & 106.59 & 61.096 & 35.601 & 27.950 & 23.941 & 20.846 & 16.489 & 11.834 & 8.027 & 4.3833 & 1.7881 & 0.4762 & 0.0763 & & & \\
\hline 14 & 107.17 & 61.004 & 35.911 & 27.231 & 23.266 & 20.412 & 17.884 & 12.950 & 9.655 & 6.3242 & 3.2457 & 1.2993 & 0.3508 & 0.0573 & & \\
\hline 15 & 107.54 & 61.048 & 36.226 & 26.865 & 22.526 & 20.158 & 17.903 & 14.461 & 10.562 & 8.0560 & 4.8995 & 2.4070 & 0.9672 & 0.2632 & 0.0435 & \\
\hline 16 & 107.74 & 61.214 & 36.481 & 26.799 & 21.847 & 19.709 & 17.795 & 15.214 & 11.863 & 8.6598 & 6.9238 & 3.6040 & 1.8474 & 0.7303 & 0.2008 & 0.0334 \\
\hline
\end{tabular}




\subsection{Treating Complete CCF Events}

As described in Section 2, the existing process uses the binomial regression method rather than the mapping technique to curve fit the fraction of complete CCF events over the total number of CCF events. This is probably due to concerns that the mapping technique might be adding too many pseudo-complete $\mathrm{CCF}$ events to other group sizes when mapping the observed complete CCF events from one group size to other group sizes. For example, Table 8 shows 34 complete CCF events for group size 2, 12 for group size 3 , and 56 for all group sizes combined. Using the mapping technique, all complete CCF events in group sizes 3-16 (i.e., $56-34=22$ ) would be mapped down with 22 pseudo-complete CCF events added to group size 2. For group size 3, all complete CCF events in group sizes 4-16 (i.e., $56-34-12=10$ ) would be mapped down, and the complete CCF events in group size 2 (which is 34 ) would be mapped up and added to group size 3 . Assuming 0.5 to be the conditional probability of failure for each component, given a nonlethal shock, $\rho, 10+34 * 0.5=27$ pseudo-complete CCF events would be added to group size 3.

The binomial regression used in Section 2 defines $P(m)$ as the probability of a CCF event being a complete failure in a particular group size, $\mathrm{m}$. It then uses the observed fractions of complete CCF failures in all group sizes and fits the data using a pre-defined function. In this study, MATLAB [21] was used for curve fitting. However, the curve fitting results for the data in Table 2 could not be reproduced using MATLAB in conjunction with the general logit function $\ln \left(\frac{P(m)}{1-P(m)}\right)=a+b m$, as per [20], or with any other pre-defined functions in MATLAB. Instead, the following function (suggested by Cory Atwood, the primary author of NUREG/CR-6823) was used to fit the curve:

$$
\ln \left(\frac{P(m)}{1-P(m)}\right)=a+b\left(1-e^{-m}\right)
$$

The results (i.e., the probability of a complete CCF event and the estimated number of complete CCF events for each group size) are listed in the last two columns of Table 10. Note that the binomial regression treatment of complete CCF events in Table 10 (and in Section 2) does not distinguish lethal shock events from nonlethal shock but complete CCF events. For lethal shocks, the impact vector is supposed to map directly (i.e., the probability of all $\mathrm{x}$ components in a system of $\mathrm{x}$ components having failed due to lethal shock is mapped directly and equals the probability of failing all y components in a system of y components). The correct process should treat lethal shock events differently from nonlethal shock but complete CCF events, i.e., the lethal shock events should be removed from the curve fitting process (do not include the lethal shock events in the No. Complete CCF Events column in Table 10), instead, the total number of lethal shock events, disregarding their group sizes, should be added to the final $n_{k}$ value in Section 3.3 and Table 11.

A review of the CCF data used in this study (1997-2015) found only three CCF events coded as lethal shock: 244-2005-0142, 263-1999-0046, and 423-2012-0501—all corresponding to a group size of 2. While the results in Table 10 are used in the following sections to estimate prior distributions, sensitivity analysis could be conducted to estimate the prior distributions via different treatment of complete CCF events (i.e., using the mapping or binomial regression methods but distinguishing lethal shocks from nonlethal ones).

Table 10. CCF data (1997-2015) with curve-fitted complete CCF events.

\begin{tabular}{|c|c|c|c|c|c|c|}
\hline $\begin{array}{c}\text { Group } \\
\text { Size }\end{array}$ & $\begin{array}{c}\text { No. Partial } \\
\text { CCF } \\
\text { Events }\end{array}$ & $\begin{array}{c}\text { No. } \\
\text { Complete } \\
\text { CCF } \\
\text { Events }\end{array}$ & $\begin{array}{c}\text { Total No. } \\
\text { CCF } \\
\text { Events }\end{array}$ & $\begin{array}{c}\text { Prob. of } \\
\text { Complete } \\
\text { CCF Event - } \\
\text { Data }\end{array}$ & $\begin{array}{c}\text { Prob. of } \\
\text { Complete CCF } \\
\text { Event - Curve } \\
\text { Fitting }\end{array}$ & $\begin{array}{c}\text { Estimated } \\
\text { No. } \\
\text { Complete } \\
\text { CCF Events }\end{array}$ \\
\hline 2 & 27 & 34 & 61 & 0.55738 & 0.51050 & 31.14031 \\
\hline 3 & 27 & 12 & 39 & 0.30769 & 0.30184 & 11.77164 \\
\hline
\end{tabular}




\begin{tabular}{|c|c|c|c|c|c|c|}
\hline $\begin{array}{c}\text { Group } \\
\text { Size }\end{array}$ & $\begin{array}{c}\text { No. Partial } \\
\text { CCF } \\
\text { Events }\end{array}$ & $\begin{array}{c}\text { No. } \\
\text { Complete } \\
\text { CCF } \\
\text { Events }\end{array}$ & $\begin{array}{c}\text { Total No. } \\
\text { CCF } \\
\text { Events }\end{array}$ & $\begin{array}{c}\text { Prob. of } \\
\text { Complete } \\
\text { CCF Event - } \\
\text { Data }\end{array}$ & $\begin{array}{c}\text { Prob. of } \\
\text { Complete CCF } \\
\text { Event - Curve } \\
\text { Fitting }\end{array}$ & $\begin{array}{c}\text { Estimated } \\
\text { No. } \\
\text { Complete } \\
\text { CCF Events }\end{array}$ \\
\hline 4 & 61 & 2 & 63 & 0.03175 & 0.17199 & 10.83554 \\
\hline 5 & 7 & 0 & 7 & 0.00000 & 0.11118 & 0.77827 \\
\hline 6 & 30 & 5 & 35 & 0.14286 & 0.08650 & 3.02750 \\
\hline 7 & 3 & 0 & 3 & 0.00000 & 0.07707 & 0.23122 \\
\hline 8 & 30 & 2 & 32 & 0.06250 & 0.07355 & 2.35374 \\
\hline 9 & 0 & 0 & 0 & NA & 0.07225 & 0.00000 \\
\hline 10 & 0 & 0 & 0 & NA & 0.07177 & 0.00000 \\
\hline 11 & 5 & 0 & 5 & 0.00000 & 0.07160 & 0.35799 \\
\hline 12 & 7 & 1 & 8 & 0.12500 & 0.07153 & 0.57226 \\
\hline 13 & 0 & 0 & 0 & NA & 0.07151 & 0.00000 \\
\hline 14 & 1 & 0 & 1 & 0.00000 & 0.07150 & 0.07150 \\
\hline 15 & 0 & 0 & 0 & NA & 0.07150 & 0.00000 \\
\hline 16 & 14 & 0 & 14 & 0.00000 & 0.07150 & 1.00094 \\
\hline Total & 212 & 56 & 268 & & & 62.14091 \\
\hline
\end{tabular}

\subsection{Estimating Prior Distributions}

\section{Adjusted $n_{k}$ Values}

Adjusted $n_{k}$ values for CCF events from 1997 through 2015 are obtained for each group size by adding the estimated number of complete CCF events in Table 10 to the final $n_{k}$ value for the partial CCF events in Table 9. For example, the estimated number of complete CCF events for a group size of 2 is 31.140 in Table 10; the $n_{2}$ value for partial CCF events for a group size of 2 in Table 9 is 31.164; the adjusted $n_{2}$ value for a group size of 2 will be $31.164+31.140=62.304$. Table 11 shows the adjusted $n_{k}$ results for group sizes 2-16, with CCF data for 1997-2015. The number of effective independent failure events $\left(n_{I}\right)$, as obtained from the CCF database website query results (see Figure 3-3), and the total number of failures $\left(n_{t}\right)$ (i.e., $n_{I}$ and $n_{k}$ ) for each group size are also presented in the table.

Table 11. Adjusted $n_{k}$ values for CCF events from 1997 through 2015.

\begin{tabular}{|c|c|c|c|c|c|c|c|c|c|c|c|c|c|c|c|c|c|c|}
\hline $\begin{array}{c}\text { Group } \\
\text { Size }\end{array}$ & $\mathrm{n}_{\mathrm{t}}$ & $\mathrm{n}_{\mathrm{I}}$ & $\mathrm{n}_{1}$ & $\mathrm{n}_{2}$ & $\mathrm{n}_{3}$ & $\mathrm{n}_{4}$ & $\mathrm{n}_{5}$ & $\mathrm{n}_{6}$ & $\mathrm{n}_{7}$ & $\mathrm{n}_{8}$ & $\mathrm{n}_{9}$ & $\mathrm{n}_{10}$ & $\mathrm{n}_{11}$ & $\mathrm{n}_{12}$ & $\mathrm{n}_{13}$ & $\mathrm{n}_{14}$ & $\mathrm{n}_{15}$ & $\mathrm{n}_{16}$ \\
\hline 2 & 3042.49 & 2864.58 & 115.61 & 62.304 & & & & & & & & & & & & & & \\
\hline 3 & 4491.83 & 4296.87 & 109.23 & 64.693 & 21.038 & & & & & & & & & & & & & \\
\hline 4 & 5947.30 & 5729.16 & 96.47 & 80.938 & 25.857 & 14.873 & & & & & & & & & & & & \\
\hline 5 & 7388.92 & 7161.45 & 96.92 & 73.190 & 39.900 & 14.539 & 2.919 & & & & & & & & & & & \\
\hline 6 & 8839.80 & 8593.74 & 98.05 & 65.654 & 46.584 & 23.001 & 8.533 & 4.237 & & & & & & & & & & \\
\hline 7 & 10283.74 & 10026.03 & 101.15 & 61.106 & 44.388 & 29.499 & 15.401 & 5.219 & 0.948 & & & & & & & & & \\
\hline 8 & 11731.02 & 11458.32 & 102.97 & 59.825 & 41.221 & 31.486 & 20.828 & 10.368 & 3.197 & 2.807 & & & & & & & & \\
\hline 9 & 13172.57 & 12890.61 & 104.47 & 59.373 & 38.811 & 31.849 & 23.262 & 14.602 & 7.206 & 2.091 & 0.298 & & & & & & & \\
\hline 10 & 14615.39 & 14322.90 & 105.31 & 59.671 & 37.235 & 31.000 & 24.661 & 17.351 & 10.623 & 5.043 & 1.393 & 0.2028 & & & & & & \\
\hline 11 & 16057.63 & 15755.19 & 105.59 & 60.457 & 36.289 & 29.832 & 25.016 & 19.214 & 13.174 & 7.876 & 3.546 & 0.9485 & 0.5007 & & & & & \\
\hline 12 & 17498.95 & 17187.48 & 105.79 & 61.357 & 35.385 & 29.051 & 24.559 & 20.390 & 15.020 & 10.190 & 5.885 & 2.5054 & 0.6629 & 0.6755 & & & & \\
\hline
\end{tabular}




\begin{tabular}{|c|c|c|c|c|c|c|c|c|c|c|c|c|c|c|c|c|c|c|}
\hline $\begin{array}{c}\text { Group } \\
\text { Size } \\
\end{array}$ & $\mathrm{n}_{\mathrm{t}}$ & $\mathrm{n}_{\mathrm{I}}$ & $\mathrm{n}_{1}$ & $\mathrm{n}_{2}$ & $\mathrm{n}_{3}$ & $\mathrm{n}_{4}$ & $\mathrm{n}_{5}$ & $\mathrm{n}_{6}$ & $\mathrm{n}_{7}$ & $\mathrm{n}_{8}$ & $\mathrm{n}_{9}$ & $\mathrm{n}_{10}$ & $\mathrm{n}_{11}$ & $\mathrm{n}_{12}$ & $\mathrm{n}_{13}$ & $\mathrm{n}_{14}$ & $\mathrm{n}_{15}$ & $\mathrm{n}_{16}$ \\
\hline 13 & 18938.87 & 18619.77 & 106.59 & 61.096 & 35.601 & 27.950 & 23.941 & 20.846 & 16.489 & 11.834 & 8.027 & 4.3833 & 1.7881 & 0.4762 & 0.0763 & & & \\
\hline 14 & 20378.89 & 20052.06 & 107.17 & 61.004 & 35.911 & 27.231 & 23.266 & 20.412 & 17.884 & 12.950 & 9.655 & 6.3242 & 3.2457 & 1.2993 & 0.3508 & 0.1288 & & \\
\hline 15 & 21818.28 & 21484.35 & 107.54 & 61.048 & 36.226 & 26.865 & 22.526 & 20.158 & 17.903 & 14.461 & 10.562 & 8.0560 & 4.8995 & 2.4070 & 0.9672 & 0.2632 & 0.0435 & \\
\hline 16 & 23258.30 & 22916.64 & 107.74 & 61.214 & 36.481 & 26.799 & 21.847 & 19.709 & 17.795 & 15.214 & 11.863 & 8.6598 & 6.9238 & 3.6040 & 1.8474 & 0.7303 & 0.2008 & 1.0343 \\
\hline
\end{tabular}

\section{Alpha Factor Mean Values}

The MLEs or mean values of alpha factors for each group size can then be calculated using Eqs. 3 and 4 . Table 12 presents the results.

Table 12. Calculated alpha factor mean values for CCF events from 1997 through 2015.

\begin{tabular}{|c|c|c|c|c|c|c|c|c|c|c|c|c|c|c|c|c|}
\hline $\begin{array}{c}\text { Group } \\
\text { Size }\end{array}$ & $\alpha_{1}$ & $\alpha_{2}$ & $\alpha_{3}$ & $\alpha_{4}$ & $\alpha_{5}$ & $\alpha_{6}$ & $\alpha_{7}$ & $\alpha_{8}$ & $\alpha_{9}$ & $\alpha_{10}$ & $\alpha_{11}$ & $\alpha_{12}$ & $\alpha_{13}$ & $\alpha_{14}$ & $\alpha_{15}$ & $\alpha_{16}$ \\
\hline 2 & 0.9795 & $2.048 \mathrm{E}-02$ & & & & & & & & & & & & & & \\
\hline 3 & 0.9809 & $1.440 \mathrm{E}-02$ & $4.684 \mathrm{E}-03$ & & & & & & & & & & & & & \\
\hline 4 & 0.9795 & $1.361 \mathrm{E}-02$ & $4.348 \mathrm{E}-03$ & $2.501 \mathrm{E}-03$ & & & & & & & & & & & & \\
\hline 5 & 0.9823 & $9.905 \mathrm{E}-03$ & $5.400 \mathrm{E}-03$ & $1.968 \mathrm{E}-03$ & $3.951 \mathrm{E}-04$ & & & & & & & & & & & \\
\hline 6 & 0.9833 & 7.427E-03 & $5.270 \mathrm{E}-03$ & $2.602 \mathrm{E}-03$ & $9.653 \mathrm{E}-04$ & $4.793 \mathrm{E}-04$ & & & & & & & & & & \\
\hline 7 & 0.9848 & 5.942E-03 & $4.316 \mathrm{E}-03$ & $2.869 \mathrm{E}-03$ & $1.498 \mathrm{E}-03$ & $5.075 \mathrm{E}-04$ & $9.214 \mathrm{E}-05$ & & & & & & & & & \\
\hline 8 & 0.9855 & $5.100 \mathrm{E}-03$ & $3.514 \mathrm{E}-03$ & $2.684 \mathrm{E}-03$ & $1.775 \mathrm{E}-03$ & $8.838 \mathrm{E}-04$ & $2.725 \mathrm{E}-04$ & $2.393 \mathrm{E}-04$ & & & & & & & & \\
\hline 9 & 0.9865 & $4.507 \mathrm{E}-03$ & $2.946 \mathrm{E}-03$ & $2.418 \mathrm{E}-03$ & $1.766 \mathrm{E}-03$ & $1.109 \mathrm{E}-03$ & $5.470 \mathrm{E}-04$ & $1.587 \mathrm{E}-04$ & $2.261 \mathrm{E}-05$ & & & & & & & \\
\hline 10 & 0.9872 & $4.083 \mathrm{E}-03$ & $2.548 \mathrm{E}-03$ & $2.121 \mathrm{E}-03$ & $1.687 \mathrm{E}-03$ & $1.187 \mathrm{E}-03$ & 7.268E-04 & $3.450 \mathrm{E}-04$ & $9.530 \mathrm{E}-05$ & $1.388 \mathrm{E}-05$ & & & & & & \\
\hline 11 & 0.9877 & $3.765 \mathrm{E}-03$ & $2.260 \mathrm{E}-03$ & $1.858 \mathrm{E}-03$ & $1.558 \mathrm{E}-03$ & $1.197 \mathrm{E}-03$ & 8.204E-04 & $4.905 \mathrm{E}-04$ & $2.208 \mathrm{E}-04$ & 5.907E-05 & $3.118 \mathrm{E}-05$ & & & & & \\
\hline 12 & 0.9882 & 3.506E-03 & $2.022 \mathrm{E}-03$ & $1.660 \mathrm{E}-03$ & $1.403 \mathrm{E}-03$ & $1.165 \mathrm{E}-03$ & 8.583E-04 & $5.823 \mathrm{E}-04$ & $3.363 \mathrm{E}-04$ & $1.432 \mathrm{E}-04$ & $3.788 \mathrm{E}-05$ & $3.860 \mathrm{E}-05$ & & & & \\
\hline 13 & 0.9888 & $3.226 \mathrm{E}-03$ & $1.880 \mathrm{E}-03$ & $1.476 \mathrm{E}-03$ & $1.264 \mathrm{E}-03$ & $1.101 \mathrm{E}-03$ & 8.706E-04 & $6.249 \mathrm{E}-04$ & $4.238 \mathrm{E}-04$ & $2.314 \mathrm{E}-04$ & $9.441 \mathrm{E}-05$ & $2.515 \mathrm{E}-05$ & $4.030 \mathrm{E}-06$ & & & \\
\hline 14 & 0.9892 & 2.993E-03 & $1.762 \mathrm{E}-03$ & $1.336 \mathrm{E}-03$ & $1.142 \mathrm{E}-03$ & $1.002 \mathrm{E}-03$ & $8.776 \mathrm{E}-04$ & $6.355 \mathrm{E}-04$ & $4.738 \mathrm{E}-04$ & $3.103 \mathrm{E}-04$ & $1.593 \mathrm{E}-04$ & $6.376 \mathrm{E}-05$ & $1.721 \mathrm{E}-05$ & $56.319 \mathrm{E}-06$ & & \\
\hline 15 & 0.9896 & 2.798E-03 & $1.660 \mathrm{E}-03$ & $1.231 \mathrm{E}-03$ & $1.032 \mathrm{E}-03$ & $9.239 \mathrm{E}-04$ & 8.206E-04 & $6.628 \mathrm{E}-04$ & $4.841 \mathrm{E}-04$ & $3.692 \mathrm{E}-04$ & $2.246 \mathrm{E}-04$ & $1.103 \mathrm{E}-04$ & $4.433 \mathrm{E}-05$ & $51.207 \mathrm{E}-05$ & $1.995 \mathrm{E}-06$ & \\
\hline 16 & 0.9899 & 2.632E-03 & $1.569 \mathrm{E}-03$ & $1.152 \mathrm{E}-03$ & 9.393E-04 & $8.474 \mathrm{E}-04$ & $7.651 \mathrm{E}-04$ & $6.541 \mathrm{E}-04$ & $5.101 \mathrm{E}-04$ & $3.723 \mathrm{E}-04$ & $2.977 \mathrm{E}-04$ & $1.550 \mathrm{E}-04$ & $7.943 \mathrm{E}-05$ & $53.140 \mathrm{E}-05$ & 8.633E-06 & $4.447 \mathrm{E}-05$ \\
\hline
\end{tabular}

\section{CalcPrior Code and Prior Distributions}

Adjusted $n_{k}$ values, including the number of effective independent failure events $\left(n_{I}\right)$ in Table 11, are used as input to the computer code CalcPrior to estimate the industry-wide prior distributions with parameters $\alpha$ and $\beta$. The CalcPrior code was first developed in early 2000, then re-coded with modern computer language for this study. Figure 3-4 shows the CalcPrior code homepage. Figure 3-5 shows the needed input to the code to calculate the prior distributions. Such input includes the prior name, independent event count, average CCCG size, description of the prior, and $n_{k}$ values for each group size.

The total independent event count (called the effective independent event count or unadjusted independent count in the CCF Database website), adjusted independent event count, and unadjusted $n_{k}$ values for each group size are included in the CCF Database website querying results. The average CCCG size can be calculated from Eq. 1 . The $n_{k}$ values for each group size can be adjusted after the proper treatment of complete CCF events. These values can be input to the CalcPrior code via the following .csv file format:

PriorName, PriorDescription,,,,,,,,,,,,,

TotalIndependentEventCount, AverageGroupSize,,,,,,,,,,,, 
$n_{1,} n_{2},,,,,,,,,,$,

$n_{1,} n_{2,} n_{3,,,,,,,,,,,,,}$

$n_{1,} n_{2}, n_{3,} n_{4,,,,,,,,,,,}$

The $n_{I}$ values and $n_{t}$ values for each group size will be automatically calculated by the code in accordance with the input values. The code can then estimate prior distributions, based on the constrained noninformative and Dirichlet methodology (refer to [20]). Figure 3-6 shows the CalcPrior code results for the prior distribution parameters, which can be output to Table 13.

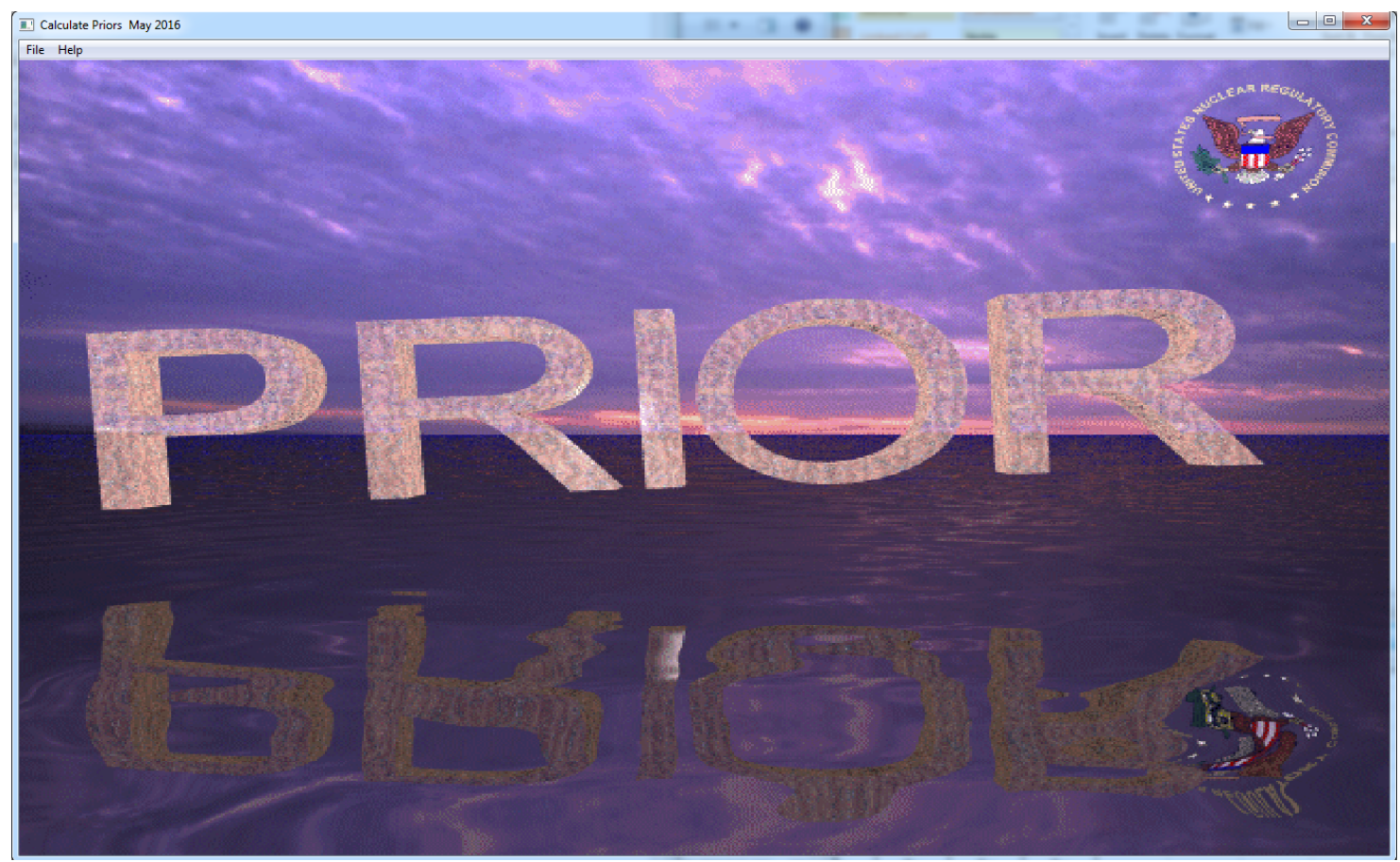

Figure 3-4. Homepage of the CalcPrior code for estimating alpha factor prior distributions. 


\begin{tabular}{|c|c|c|c|c|c|c|c|c|c|c|}
\hline \multicolumn{2}{|c|}{ Define/View Prior } & $\theta$ & & & & & & & \multicolumn{2}{|c|}{$x$} \\
\hline Name & \multicolumn{2}{|l|}{ CCF2015-ALL } & \multicolumn{2}{|c|}{ Independent Event Count } & \multicolumn{2}{|c|}{7492.800} & \multicolumn{2}{|c|}{ Average CCCG Size } & 5.231 & \\
\hline \multicolumn{11}{|c|}{ Description Priors for 1997.2015} \\
\hline CCCG Size & Sum of $N$ & Adj. Ind. Events & N1 & N2 & N3 & N4 & N5 & N6 & N7 & A \\
\hline 2 & 3042.6817 & 2864.77 & 115.6100 & 62.3040 & & & & & & \\
\hline 3 & 4492.1126 & 4297.15 & 109.2300 & 64.6930 & 21.0380 & & & & & \\
\hline 4 & 5947.6735 & 5729.54 & 96.4700 & 80.9380 & 25.8570 & 14.8730 & & & & \\
\hline 5 & 7389.3873 & 7161.92 & 96.9200 & 73.1900 & 39.9000 & 14.5390 & 2.9190 & & & \\
\hline 6 & 8840.3622 & 8594.30 & 98.0500 & 65.6540 & 46.5840 & 23.0010 & 8.5330 & 4.2370 & & \\
\hline 7 & 10284.3981 & 10026.69 & 101.1500 & 61.1060 & 44.3880 & 29.4990 & 15.4010 & 5.2190 & 0.948 & $=$ \\
\hline 8 & 11731.7729 & 11459.07 & 102.9700 & 59.8250 & 41.2210 & 31.4860 & 20.8280 & 10.3680 & 3.19 & \\
\hline 9 & 13173.4168 & 12891.45 & 104.4700 & 59.3730 & 38.8110 & 31.8490 & 23.2620 & 14.6020 & 7.200 & \\
\hline 10 & 14616.3285 & 14323.84 & 105.3100 & 59.6710 & 37.2350 & 31.0000 & 24.6610 & 17.3510 & 10.60 & \\
\hline 11 & 16058.6657 & 15756.22 & 105.5900 & 60.4570 & 36.2890 & 29.8320 & 25.0160 & 19.2140 & 13.17 & \\
\hline 12 & 17500.0772 & 17188.61 & 105.7900 & 61.3570 & 35.3850 & 29.0510 & 24.5590 & 20.3900 & 15.02 & \\
\hline 13 & 18940.0882 & 18620.99 & 106.5900 & 61.0960 & 35.6010 & 27.9500 & 23.9410 & 20.8460 & 16.48 & 1 \\
\hline 14 & 20380.2059 & 20053.37 & 107.1700 & 61.0040 & 35.9110 & 27.2310 & 23.2660 & 20.4120 & 17.88 & \\
\hline \multicolumn{9}{|c|}{$1{ }^{\prime \prime \prime I}$} & 1 & - \\
\hline Calculate & & & Sa: & Car & icel & & & & & \\
\hline
\end{tabular}

Figure 3-5. Input to the CalcPrior code for estimating alpha factor prior distributions.

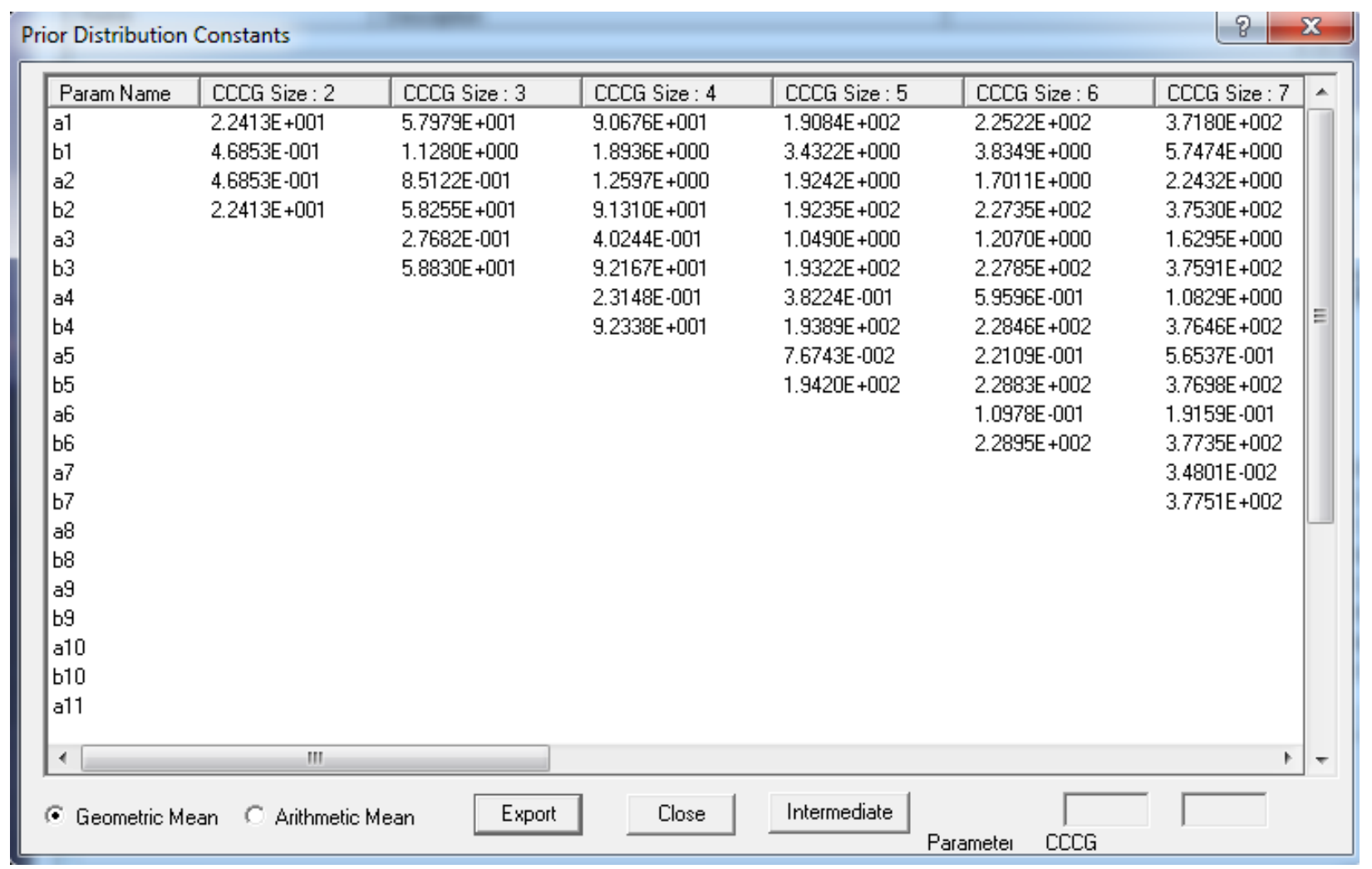

Figure 3-6. Prior distribution parameters calculated by the CalcPrior code. 
Table 13. Estimated CCF industry-wide prior distributions with CCF events from 1997 through 2015.

\begin{tabular}{|c|c|c|c|c|c|c|c|c|c|c|c|c|c|c|c|c|}
\hline $\begin{array}{c}\text { Group } \\
\text { Size }\end{array}$ & $\alpha_{1}$ & $\mathrm{~b}_{1}$ & $\alpha_{2}$ & $\mathrm{~b}_{2}$ & $\alpha_{3}$ & $b_{3}$ & $\alpha_{4}$ & $\mathrm{~b}_{4}$ & $\alpha_{5}$ & $b_{5}$ & $\alpha_{6}$ & $\mathrm{~b}_{6}$ & $\alpha_{7}$ & $b_{7}$ & $\alpha_{8}$ & $\mathrm{~b}_{8}$ \\
\hline 2 & $2.2413 \mathrm{E}+01$ & 4.6853E-01 & 4.6853E-01 & $2.2413 \mathrm{E}+01$ & & & & & & & & & & & & \\
\hline 3 & 5.7979E+01 & $1.1280 \mathrm{E}+00$ & $8.5122 \mathrm{E}-01$ & $5.8255 \mathrm{E}+01$ & 2.7682E-01 & $5.8830 \mathrm{E}+01$ & & & & & & & & & & \\
\hline 4 & $9.0676 \mathrm{E}+01$ & $1.8936 \mathrm{E}+00$ & $1.2597 \mathrm{E}+00$ & $9.1310 \mathrm{E}+01$ & 4.0244E-01 & $9.2167 \mathrm{E}+01$ & 2.3148E-01 & $9.2338 \mathrm{E}+01$ & & & & & & & & \\
\hline 5 & $1.9084 \mathrm{E}+02$ & 3.4322E+00 & $1.9242 \mathrm{E}+00$ & $1.9235 \mathrm{E}+02$ & $1.0490 \mathrm{E}+00$ & $1.9322 \mathrm{E}+02$ & 3.8224E-01 & $1.9389 \mathrm{E}+02$ & 7.6743E-02 & $1.9420 \mathrm{E}+02$ & & & & & & \\
\hline 6 & $2.2522 \mathrm{E}+02$ & $3.8349 \mathrm{E}+00$ & $1.7011 \mathrm{E}+00$ & $2.2735 \mathrm{E}+02$ & $1.2070 \mathrm{E}+00$ & $2.2785 \mathrm{E}+02$ & 5.9596E-01 & $2.2846 \mathrm{E}+02$ & $2.2109 \mathrm{E}-01$ & $2.2883 \mathrm{E}+02$ & $1.0978 \mathrm{E}-01$ & $2.2895 \mathrm{E}+02$ & & & & \\
\hline 7 & $3.7180 \mathrm{E}+02$ & $5.7474 \mathrm{E}+00$ & $2.2432 \mathrm{E}+00$ & $3.7530 \mathrm{E}+02$ & $1.6295 \mathrm{E}+00$ & $3.7591 \mathrm{E}+02$ & $1.0829 \mathrm{E}+00$ & $3.7646 \mathrm{E}+02$ & 5.6537E-01 & $3.7698 \mathrm{E}+02$ & $1.9159 \mathrm{E}-01$ & $3.7735 \mathrm{E}+02$ & $3.4801 \mathrm{E}-02$ & $3.7751 \mathrm{E}+02$ & & \\
\hline 8 & 3.9002E+02 & $5.7256 \mathrm{E}+00$ & 2.0181E+00 & $3.9373 \mathrm{E}+02$ & $1.3905 \mathrm{E}+00$ & $3.9436 \mathrm{E}+02$ & $1.0621 \mathrm{E}+00$ & $3.9469 \mathrm{E}+02$ & 7.0259E-01 & $3.9505 \mathrm{E}+02$ & 3.4974E-01 & 3.9540E+02 & $1.0784 \mathrm{E}-01$ & $3.9564 \mathrm{E}+02$ & 9.4689E-02 & $3.9565 \mathrm{E}+02$ \\
\hline 9 & $6.3746 \mathrm{E}+02$ & $8.7061 \mathrm{E}+00$ & $2.9123 \mathrm{E}+00$ & $6.4325 \mathrm{E}+02$ & $1.9037 \mathrm{E}+00$ & $6.4426 \mathrm{E}+02$ & $1.5622 \mathrm{E}+00$ & $6.4460 \mathrm{E}+02$ & $1.1410 \mathrm{E}+00$ & $6.4502 \mathrm{E}+02$ & 7.1624E-01 & $6.4545 \mathrm{E}+02$ & $3.5346 \mathrm{E}-01$ & $6.4581 \mathrm{E}+02$ & $1.0257 \mathrm{E}-01$ & $6.4606 \mathrm{E}+02$ \\
\hline 10 & 7.8579E+02 & $1.0194 \mathrm{E}+01$ & $3.2496 \mathrm{E}+00$ & $7.9274 \mathrm{E}+02$ & $2.0278 \mathrm{E}+00$ & $7.9396 \mathrm{E}+02$ & $1.6882 \mathrm{E}+00$ & $7.9430 \mathrm{E}+02$ & $1.3430 \mathrm{E}+00$ & $7.9465 \mathrm{E}+02$ & 9.4492E-01 & $7.9504 \mathrm{E}+02$ & $5.7852 \mathrm{E}-01$ & $7.9541 \mathrm{E}+02$ & $2.7464 \mathrm{E}-01$ & $7.9571 \mathrm{E}+02$ \\
\hline 11 & $8.3890 \mathrm{E}+02$ & $1.0411 \mathrm{E}+01$ & $3.1974 \mathrm{E}+00$ & $8.4611 \mathrm{E}+02$ & $1.9192 \mathrm{E}+00$ & $8.4739 \mathrm{E}+02$ & $1.5777 \mathrm{E}+00$ & $8.4773 \mathrm{E}+02$ & $1.3230 \mathrm{E}+00$ & $8.4799 \mathrm{E}+02$ & $1.0162 \mathrm{E}+00$ & $8.4829 \mathrm{E}+02$ & 6.9674E-01 & $8.4861 \mathrm{E}+02$ & 4.1654E-01 & 8.4889E+02 \\
\hline 12 & $9.5624 \mathrm{E}+02$ & $1.1373 \mathrm{E}+01$ & $3.3926 \mathrm{E}+00$ & $9.6422 \mathrm{E}+02$ & $1.9565 \mathrm{E}+00$ & $9.6566 \mathrm{E}+02$ & $1.6063 \mathrm{E}+00$ & $9.6601 \mathrm{E}+02$ & $1.3579 \mathrm{E}+00$ & $9.6626 \mathrm{E}+02$ & $1.1274 \mathrm{E}+00$ & $9.6649 \mathrm{E}+02$ & 8.3049E-01 & $9.6679 \mathrm{E}+02$ & 5.6343E-01 & $9.6705 \mathrm{E}+02$ \\
\hline 13 & $1.3364 \mathrm{E}+03$ & $1.5165 \mathrm{E}+01$ & $4.3599 \mathrm{E}+00$ & $1.3472 \mathrm{E}+03$ & $2.5405 \mathrm{E}+00$ & $1.3491 \mathrm{E}+03$ & $1.9946 \mathrm{E}+00$ & $1.3496 \mathrm{E}+03$ & $1.7085 \mathrm{E}+00$ & $1.3499 \mathrm{E}+03$ & $1.4876 \mathrm{E}+00$ & $1.3501 \mathrm{E}+03$ & $1.1767 \mathrm{E}+00$ & $1.3504 \mathrm{E}+03$ & 8.4449E-01 & $1.3508 \mathrm{E}+03$ \\
\hline 14 & $1.4630 \mathrm{E}+03$ & $1.5941 \mathrm{E}+01$ & $4.4270 \mathrm{E}+00$ & $1.4746 \mathrm{E}+03$ & $2.6060 \mathrm{E}+00$ & $1.4764 \mathrm{E}+03$ & $1.9761 \mathrm{E}+00$ & $1.4770 \mathrm{E}+03$ & $1.6884 \mathrm{E}+00$ & $1.4773 \mathrm{E}+03$ & $1.4813 \mathrm{E}+00$ & $1.4775 \mathrm{E}+03$ & $1.2978 \mathrm{E}+00$ & $1.4777 \mathrm{E}+03$ & 9.3977E-01 & $1.4780 \mathrm{E}+03$ \\
\hline 15 & $1.7967 \mathrm{E}+03$ & $1.8836 \mathrm{E}+01$ & $5.0795 \mathrm{E}+00$ & $1.8104 \mathrm{E}+03$ & $3.0142 \mathrm{E}+00$ & $1.8125 \mathrm{E}+03$ & 2.2353E+00 & $1.8133 \mathrm{E}+03$ & $1.8743 \mathrm{E}+00$ & $1.8136 \mathrm{E}+03$ & $1.6773 \mathrm{E}+00$ & $1.8138 \mathrm{E}+03$ & $1.4896 \mathrm{E}+00$ & $1.8140 \mathrm{E}+03$ & $1.2032 \mathrm{E}+00$ & $1.8143 \mathrm{E}+03$ \\
\hline 16 & $1.6355 \mathrm{E}+03$ & $1.6615 \mathrm{E}+01$ & $4.3479 \mathrm{E}+00$ & $1.6478 \mathrm{E}+03$ & $2.5912 \mathrm{E}+00$ & $1.6495 \mathrm{E}+03$ & $1.9035 E+00$ & $1.6502 \mathrm{E}+03$ & $1.5518 \mathrm{E}+00$ & $1.6506 \mathrm{E}+03$ & $1.3999 \mathrm{E}+00$ & $1.6507 \mathrm{E}+03$ & $1.2640 \mathrm{E}+00$ & $1.6508 \mathrm{E}+03$ & $1.0806 \mathrm{E}+00$ & $1.6510 \mathrm{E}+03$ \\
\hline
\end{tabular}

\begin{tabular}{|c|c|c|c|c|c|c|c|c|c|c|c|c|c|c|c|c|}
\hline $\begin{array}{l}\text { Group } \\
\text { Size }\end{array}$ & $\alpha_{9}$ & $\mathrm{~b}_{9}$ & $\alpha_{10}$ & $b_{10}$ & $\alpha_{11}$ & $b_{11}$ & $\alpha_{12}$ & $b_{12}$ & $\alpha_{13}$ & $b_{13}$ & $\alpha_{14}$ & $b_{14}$ & $\alpha_{15}$ & $b_{15}$ & $\alpha_{16}$ & $b_{16}$ \\
\hline 9 & $1.4617 \mathrm{E}-02$ & $6.4615 \mathrm{E}+02$ & & & & & & & & & & & & & & \\
\hline 10 & 7.5861E-02 & $7.9591 \mathrm{E}+02$ & $1.1044 \mathrm{E}-02$ & $7.9598 \mathrm{E}+02$ & & & & & & & & & & & & \\
\hline 11 & $1.8754 \mathrm{E}-01$ & $8.4912 \mathrm{E}+02$ & 5.0164E-02 & $8.4926 \mathrm{E}+02$ & 2.6481E-02 & $8.4928 \mathrm{E}+02$ & & & & & & & & & & \\
\hline 12 & 3.2539E-01 & $9.6729 \mathrm{E}+02$ & $1.3853 \mathrm{E}-01$ & $9.6748 \mathrm{E}+02$ & 3.6653E-02 & $9.6758 \mathrm{E}+02$ & 3.7350E-02 & $9.6758 \mathrm{E}+02$ & & & & & & & & \\
\hline 13 & 5.7282E-01 & $1.3510 \mathrm{E}+03$ & 3.1280E-01 & $1.3513 \mathrm{E}+03$ & $1.2760 \mathrm{E}-01$ & $1.3515 \mathrm{E}+03$ & 3.3982E-02 & $1.3516 \mathrm{E}+03$ & 5.4449E-03 & $1.3516 \mathrm{E}+03$ & & & & & & \\
\hline 14 & 7.0066E-01 & $1.4783 \mathrm{E}+03$ & 4.5894E-01 & $1.4785 \mathrm{E}+03$ & 2.3554E-01 & $1.4787 \mathrm{E}+03$ & 9.4289E-02 & $1.4789 \mathrm{E}+03$ & 2.5457E- 02 & $1.4790 \mathrm{E}+03$ & 9.3469E-03 & $1.4790 \mathrm{E}+03$ & & & & \\
\hline 15 & 8.7881E-01 & $1.8146 \mathrm{E}+03$ & 6.7030E-01 & $1.8148 \mathrm{E}+03$ & 4.0766E-01 & $1.8151 \mathrm{E}+03$ & 2.0028E-01 & $1.8153 \mathrm{E}+03$ & $8.0476 \mathrm{E}-02$ & $1.8154 \mathrm{E}+03$ & $2.1900 \mathrm{E}-02$ & $1.8155 \mathrm{E}+03$ & 3.6194E-03 & $1.8155 \mathrm{E}+03$ & & \\
\hline 16 & 8.4261E-01 & $1.6513 \mathrm{E}+03$ & 6.1509E-01 & $1.6515 \mathrm{E}+03$ & 4.9179E-01 & $1.6516 \mathrm{E}+03$ & 2.5599E-01 & $1.6519 \mathrm{E}+03$ & $1.3122 \mathrm{E}-01$ & $1.6520 \mathrm{E}+03$ & 5.1872E-02 & $1.6521 \mathrm{E}+03$ & $1.4263 \mathrm{E}-02$ & $1.6521 \mathrm{E}+03$ & 7.3465E-02 & $1.6520 \mathrm{E}+03$ \\
\hline
\end{tabular}




\section{DEVELOPING GENERIC PRIOR DISTRIBUTIONS FOR CAUSAL ALPHA FACTORS}

While the AFM is widely utilized in PRAs, including the Standardized Plant Analysis Risk (SPAR) models, industry has expressed some concerns over applying the AFM in event and condition assessments (ECAs). For example, the AFM is not causal-based, as the methodology does not explicitly incorporate causes of failure. So, when using the AFM in ECA, the conditional CCF probabilities in the AFM do not acknowledge other causes that may be included in the CCF probabilities but not affect the component that fails, and as such would bias the risk evaluation for the event or condition. To address this concern, NRC asked INL to conduct a feasibility study in 2014 to aid NRC in investigating alternative CCF models potentially usable for event assessment in the SPAR models. The feasibility study suggests that the CAFM — or Partial Alpha Factor Model (PAFM), as referred to in the study report [22] — could be used in the SPAR models to replace the current AFM for ECA. To implement the CAFM in the SPAR models, new generic causal prior distributions must be developed for the different $\mathrm{CCF}$ cause groups defined in the feasibility study. The feasibility study recommends using five CCF cause groups in the CAFM: Component (GC), Design (GD), Environment (GE), Human (GH), and Other (GO). Table 14 shows the five recommended CCF cause groups, along with the CCF failure causes (failure cause codes, descriptions, and meaning) for each group. These CCF cause groups and failure causes align with the current CCF database categorization. The same process described in Section 3 for alpha factor prior distribution updating is used to access CCF data based on a specific CCF cause, treat complete CCF events separately, and estimate prior distributions using adjusted $n_{k}$ values and the CalcPrior program.

\subsection{Prior Distributions for the "Component" Cause Group (GC)}

To estimate prior distributions for the GC (i.e., Component) CCF cause group, the following selection criteria are defined in the CCF Database website:

- Type of CCF Event Level: All Level CCF Events

- CCF Event Type: CCF Events Only

- Date Range: 1997-2015

- Filter Independent Events by Selected Cause(s): True

- Shock Criteria: All Events

- Redundancy Range: Minimum = 2, Maximum = 16

- Bayesian Update Method: Mean Method

- Failure Modes: select all failure modes except Setpoint

- CCF Categories: Cause $\rightarrow$ Component

A total of $61 \mathrm{CCF}$ events and 2,855.2 effective independent failure events related to the above selection criteria.

Additional criterion on CCF Categories $\rightarrow$ Degree $\rightarrow$ Almost/Partial or Complete is used to obtain the partial/complete $\mathrm{CCF}$ events, as required in the existing process. The unmapped/mapped impact vectors are also acquired from the CCF Database website. The mapped impact vectors for partial CCF events for each group size obtained from the website are used directly in the study. 
Table 14. CCF cause groups recommended in the alternative CCF model feasibility study.

\begin{tabular}{|c|c|c|c|}
\hline $\begin{array}{l}\text { CCF Cause } \\
\text { Group }\end{array}$ & $\begin{array}{l}\text { CCF } \\
\text { Cause } \\
\text { Code }\end{array}$ & Failure Cause Description & Failure Cause Meaning \\
\hline \multirow{3}{*}{$\begin{array}{l}\text { Component } \\
\text { (GC) }\end{array}$} & IC & $\begin{array}{l}\text { Internal to component, } \\
\text { piece-part }\end{array}$ & $\begin{array}{l}\text { Used when the cause of a failure is a non-specific result } \\
\text { of a failure internal to the component that failed, other } \\
\text { than aging or wear. }\end{array}$ \\
\hline & IQ & Setpoint drift & $\begin{array}{l}\text { Used when the cause of a failure is the result of setpoint } \\
\text { drift or adjustment. }\end{array}$ \\
\hline & IW & Age/wear & $\begin{array}{l}\text { Used when the cause of a failure is a non-specific aging } \\
\text { or wear issue. }\end{array}$ \\
\hline \multirow{3}{*}{ Design (GD) } & DC & $\begin{array}{l}\text { Construction installation error } \\
\text { or inadequacy }\end{array}$ & $\begin{array}{l}\text { Used when a construction or installation error is made } \\
\text { during the original or modification installation. This } \\
\text { includes specification of an incorrect component or } \\
\text { material. }\end{array}$ \\
\hline & $\mathrm{DE}$ & Design error or inadequacy & Used when a design error is made. \\
\hline & $\mathrm{DM}$ & $\begin{array}{l}\text { Manufacturing error or } \\
\text { inadequacy }\end{array}$ & $\begin{array}{l}\text { Used when a manufacturing error is made during } \\
\text { component manufacture. }\end{array}$ \\
\hline \multirow{3}{*}{$\begin{array}{l}\text { Environment } \\
\quad(\mathrm{GE})\end{array}$} & EA & Ambient environmental stress & $\begin{array}{l}\text { Used when the cause of a failure is the result of an } \\
\text { environmental condition from the location of the } \\
\text { component. }\end{array}$ \\
\hline & $\mathrm{EE}$ & Extreme environmental stress & $\begin{array}{l}\text { Used when the cause of a failure is the result of an } \\
\text { environmental condition that places a higher-than- } \\
\text { expected load on the equipment and is transitory in } \\
\text { nature. }\end{array}$ \\
\hline & IE & Internal environment & $\begin{array}{l}\text { Internal environment led to the failure. Debris/foreign } \\
\text { material as well as an operating medium chemistry } \\
\text { issue. }\end{array}$ \\
\hline \multirow{4}{*}{ Human (GH) } & HA & Accidental human action & $\begin{array}{l}\text { Used when a human error (during the performance of } \\
\text { an activity) results in an unintentional or undesired } \\
\text { action. }\end{array}$ \\
\hline & $\mathrm{HM}$ & Inadequate maintenance & $\begin{array}{l}\text { Used when a human error (during the performance of } \\
\text { maintenance) results in an unintentional or undesired } \\
\text { action. }\end{array}$ \\
\hline & HP & Human action procedure & $\begin{array}{l}\text { Used when the procedure is not followed or is } \\
\text { incorrect. For example, when a missed or incorrect step } \\
\text { in a surveillance procedure results in component } \\
\text { failure. }\end{array}$ \\
\hline & PA & Inadequate procedure & $\begin{array}{l}\text { Used when a failure results from an inadequate } \\
\text { operating or maintenance procedure. }\end{array}$ \\
\hline
\end{tabular}




\begin{tabular}{|c|c|l|l|}
\hline $\begin{array}{c}\text { CCF Cause } \\
\text { Group }\end{array}$ & $\begin{array}{c}\text { CCF } \\
\text { Cause } \\
\text { Code }\end{array}$ & Failure Cause Description & \multicolumn{1}{|c|}{ Failure Cause Meaning } \\
\hline \multirow{2}{*}{ Other (GO) } & EC & State of other component & $\begin{array}{l}\text { Used when a failure results from a component state not } \\
\text { associated with the component that failed. For example, } \\
\text { diesel failure due to no fuel in the fuel storage tanks. }\end{array}$ \\
\cline { 2 - 4 } & OT & Other & $\begin{array}{l}\text { Used when the cause of a failure is provided but does } \\
\text { not meet any of the descriptions. }\end{array}$ \\
\cline { 2 - 4 } & OK & Unknown & Used when the cause of the failure is unknown. \\
\hline
\end{tabular}

Table 15 shows the number of partial CCF events, the number of complete CCF events, and the total number of CCF events for the GC cause group. The same binomial regression treatment used for complete CCF events was conducted. The estimated number of complete CCF events for each group size is listed in Table 15, as well.

Table 15. CCF data for GC cause group from 1997 through 2015.

\begin{tabular}{|c|c|c|c|c|c|c|}
\hline $\begin{array}{c}\text { Group } \\
\text { Size }\end{array}$ & $\begin{array}{c}\text { No. Partial } \\
\text { CCF } \\
\text { Events }\end{array}$ & $\begin{array}{c}\text { No. } \\
\text { Complete } \\
\text { CCF } \\
\text { Events }\end{array}$ & $\begin{array}{c}\text { Total No. } \\
\text { CCF } \\
\text { Events }\end{array}$ & $\begin{array}{c}\text { Prob. of } \\
\text { Complete CCF } \\
\text { Event - Data }\end{array}$ & $\begin{array}{c}\text { Prob. of } \\
\text { Complete CCF } \\
\text { Event - Curve } \\
\text { Fitting }\end{array}$ & $\begin{array}{c}\text { Estimated } \\
\text { No. of } \\
\text { Complete } \\
\text { CCF Events }\end{array}$ \\
\hline 2 & 11 & 5 & 16 & 0.31250 & 0.39631 & 6.34089 \\
\hline 3 & 3 & 2 & 5 & 0.40000 & 0.21374 & 1.06870 \\
\hline 4 & 14 & 0 & 14 & 0.00000 & 0.11532 & 1.61442 \\
\hline 5 & 1 & 0 & 1 & 0.00000 & 0.07261 & 0.07261 \\
\hline 6 & 8 & 3 & 11 & 0.27273 & 0.05584 & 0.61426 \\
\hline 7 & 0 & 0 & 0 & NA & 0.04952 & 0.00000 \\
\hline 8 & 11 & 0 & 11 & 0.00000 & 0.04717 & 0.51890 \\
\hline 9 & 0 & 0 & 0 & NA & 0.04631 & 0.00000 \\
\hline 10 & 0 & 0 & 0 & NA & 0.04599 & 0.00000 \\
\hline 11 & 2 & 0 & 2 & 0.00000 & 0.04587 & 0.09174 \\
\hline 12 & 0 & 0 & 0 & NA & 0.04583 & 0.00000 \\
\hline 13 & 0 & 0 & 0 & NA & 0.04581 & 0.00000 \\
\hline 14 & 0 & 0 & 0 & NA & 0.04580 & 0.00000 \\
\hline 15 & 0 & 0 & 0 & NA & 0.04580 & 0.00000 \\
\hline 16 & 1 & 0 & 1 & 0.00000 & 0.04580 & 0.04580 \\
\hline Total & 51 & 10 & 61 & & & 10.36732 \\
\hline
\end{tabular}

Table 16 shows the mapped impact vectors for the partial CCF events in the GC cause groups sized $2-16$, as obtained from the CCF Database website. Table 17 shows the adjusted $n_{k}$ results for CCF events in GC cause groups sized 2-16, after adding the estimated number of complete CCF events. The MLEs or mean values of alpha factors for GC cause groups of each size are then calculated, while the CalcPrior code is used to estimate the prior distributions for causal alpha factors pertaining to the GC cause group. Table 18 and Table 19 show the mean values and the distributions results, respectively. 
Table 16. nk values for the partial CCF events in the GC cause group from 1997 through 2015.

\begin{tabular}{|c|c|c|c|c|c|c|c|c|c|c|c|c|c|c|c|c|}
\hline $\begin{array}{c}\text { Group } \\
\text { Size } \\
\end{array}$ & $\mathrm{n}_{1}$ & $\mathrm{n}_{2}$ & $\mathrm{n}_{3}$ & $\mathrm{n}_{4}$ & $\mathrm{n}_{5}$ & $\mathrm{n}_{6}$ & $\mathrm{n}_{7}$ & $\mathrm{n}_{8}$ & $\mathrm{n}_{9}$ & $\mathrm{n}_{10}$ & $\mathrm{n}_{11}$ & $\mathrm{n}_{12}$ & $\mathrm{n}_{13}$ & $\mathrm{n}_{14}$ & $\mathrm{n}_{15}$ & $\mathrm{n}_{16}$ \\
\hline 2 & 28.952 & 6.780 & & & & & & & & & & & & & & \\
\hline 3 & 30.077 & 13.350 & 2.330 & & & & & & & & & & & & & \\
\hline 4 & 27.723 & 18.633 & 5.316 & 1.017 & & & & & & & & & & & & \\
\hline 5 & 27.477 & 18.744 & 8.284 & 3.147 & 0.532 & & & & & & & & & & & \\
\hline 6 & 26.998 & 18.665 & 9.944 & 4.852 & 1.938 & 0.286 & & & & & & & & & & \\
\hline 7 & 28.048 & 17.059 & 10.491 & 6.515 & 3.230 & 1.189 & 0.160 & & & & & & & & & \\
\hline 8 & 28.880 & 16.218 & 10.375 & 7.248 & 4.628 & 2.111 & 0.750 & 0.091 & & & & & & & & \\
\hline 9 & 28.939 & 16.431 & 9.947 & 7.683 & 5.404 & 3.115 & 1.469 & 0.475 & 0.053 & & & & & & & \\
\hline 10 & 28.924 & 16.622 & 9.761 & 7.681 & 5.918 & 3.932 & 2.216 & 1.021 & 0.303 & 0.0312 & & & & & & \\
\hline 11 & 28.839 & 16.814 & 9.719 & 7.530 & 6.145 & 4.542 & 2.907 & 1.612 & 0.710 & 0.1946 & 0.0189 & & & & & \\
\hline 12 & 28.931 & 16.687 & 9.861 & 7.373 & 6.165 & 4.928 & 3.495 & 2.186 & 1.182 & 0.4938 & 0.1262 & 0.0116 & & & & \\
\hline 13 & 28.998 & 16.574 & 10.010 & 7.279 & 6.076 & 5.114 & 3.944 & 2.712 & 1.661 & 0.8692 & 0.3445 & 0.0826 & 0.0072 & & & \\
\hline 14 & 29.042 & 16.483 & 10.140 & 7.251 & 5.954 & 5.150 & 4.237 & 3.159 & 2.120 & 1.2684 & 0.6406 & 0.2412 & 0.0546 & 0.0046 & & \\
\hline 15 & 29.063 & 16.418 & 10.240 & 7.276 & 5.849 & 5.094 & 4.387 & 3.502 & 2.537 & 1.6642 & 0.9715 & 0.4730 & 0.1695 & 0.0363 & 0.0029 & \\
\hline 16 & 29.062 & 16.379 & 10.309 & 7.333 & 5.781 & 4.999 & 4.425 & 3.730 & 2.889 & 2.0406 & 1.3103 & 0.7457 & 0.3501 & 0.1196 & 0.0243 & 0.0019 \\
\hline
\end{tabular}

Table 17. Adjusted nk values for CCF events in the GC cause group from 1997 through 2015.

\begin{tabular}{|c|c|c|c|c|c|c|c|c|c|c|c|c|c|c|c|c|c|c|}
\hline $\begin{array}{c}\text { Group } \\
\text { Size }\end{array}$ & $\mathrm{n}_{\mathrm{t}}$ & $\mathrm{n}_{\mathrm{I}}$ & $\mathrm{n}_{1}$ & $\mathrm{n}_{2}$ & $\mathrm{n}_{3}$ & $\mathrm{n}_{4}$ & $\mathrm{n}_{5}$ & $\mathrm{n}_{6}$ & $\mathrm{n}_{7}$ & $\mathrm{n}_{8}$ & $\mathrm{n}_{9}$ & $\mathrm{n}_{10}$ & $\mathrm{n}_{11}$ & $\mathrm{n}_{12}$ & $\mathrm{n}_{13}$ & $\mathrm{n}_{14}$ & $\mathrm{n}_{15}$ & $\mathrm{n}_{16}$ \\
\hline 2 & 1203.19 & 1161.12 & 28.952 & 13.121 & & & & & & & & & & & & & & \\
\hline 3 & 1788.50 & 1741.67 & 30.077 & 13.350 & 3.399 & & & & & & & & & & & & & \\
\hline 4 & 2376.53 & 2322.23 & 27.723 & 18.633 & 5.316 & 2.631 & & & & & & & & & & & & \\
\hline 5 & 2961.05 & 2902.79 & 27.477 & 18.744 & 8.284 & 3.147 & 0.605 & & & & & & & & & & & \\
\hline 6 & 3546.64 & 3483.34 & 26.998 & 18.665 & 9.944 & 4.852 & 1.938 & 0.901 & & & & & & & & & & \\
\hline 7 & 4130.59 & 4063.90 & 28.048 & 17.059 & 10.491 & 6.515 & 3.230 & 1.189 & 0.160 & & & & & & & & & \\
\hline 8 & 4715.28 & 4644.46 & 28.880 & 16.218 & 10.375 & 7.248 & 4.628 & 2.111 & 0.750 & 0.610 & & & & & & & & \\
\hline 9 & 5298.54 & 5225.02 & 28.939 & 16.431 & 9.947 & 7.683 & 5.404 & 3.115 & 1.469 & 0.475 & 0.053 & & & & & & & \\
\hline 10 & 5881.98 & 5805.57 & 28.924 & 16.622 & 9.761 & 7.681 & 5.918 & 3.932 & 2.216 & 1.021 & 0.303 & 0.031 & & & & & & \\
\hline 11 & 6465.25 & 6386.13 & 28.839 & 16.814 & 9.719 & 7.530 & 6.145 & 4.542 & 2.907 & 1.612 & 0.710 & 0.195 & 0.111 & & & & & \\
\hline 12 & 7048.13 & 6966.69 & 28.931 & 16.687 & 9.861 & 7.373 & 6.165 & 4.928 & 3.495 & 2.186 & 1.182 & 0.494 & 0.126 & 0.012 & & & & \\
\hline 13 & 7630.92 & 7547.25 & 28.998 & 16.574 & 10.010 & 7.279 & 6.076 & 5.114 & 3.944 & 2.712 & 1.661 & 0.869 & 0.344 & 0.083 & 0.007 & & & \\
\hline 14 & 8213.55 & 8127.80 & 29.042 & 16.483 & 10.140 & 7.251 & 5.954 & 5.150 & 4.237 & 3.159 & 2.120 & 1.268 & 0.641 & 0.241 & 0.055 & 0.005 & & \\
\hline 15 & 8796.04 & 8708.36 & 29.063 & 16.418 & 10.240 & 7.276 & 5.849 & 5.094 & 4.387 & 3.502 & 2.537 & 1.664 & 0.971 & 0.473 & 0.170 & 0.036 & 0.003 & \\
\hline 16 & 9378.47 & 9288.92 & 29.062 & 16.379 & 10.309 & 7.333 & 5.781 & 4.999 & 4.425 & 3.730 & 2.889 & 2.041 & 1.310 & 0.746 & 0.350 & 0.120 & 0.024 & 0.048 \\
\hline
\end{tabular}


Table 18. Calculated alpha factor mean values for CCF events in the GC cause group from 1997 through 2015.

\begin{tabular}{|c|c|c|c|c|c|c|c|c|c|c|c|c|c|c|c|c|}
\hline $\begin{array}{c}\text { Group } \\
\text { Size }\end{array}$ & $\alpha_{1}$ & $\alpha_{2}$ & $\alpha_{3}$ & $\alpha_{4}$ & $\alpha_{5}$ & $\alpha_{6}$ & $\alpha_{7}$ & $\alpha_{8}$ & $\alpha_{9}$ & $\alpha_{10}$ & $\alpha_{11}$ & $\alpha_{12}$ & $\alpha_{13}$ & $\alpha_{14}$ & $\alpha_{15}$ & $\alpha_{16}$ \\
\hline 2 & 0.9891 & $1.091 \mathrm{E}-02$ & & & & & & & & & & & & & & \\
\hline 3 & 0.9906 & 7.464E-03 & $1.900 \mathrm{E}-03$ & & & & & & & & & & & & & \\
\hline 4 & 0.9888 & $7.840 \mathrm{E}-03$ & 2.237E-03 & $1.107 \mathrm{E}-03$ & & & & & & & & & & & & \\
\hline 5 & 0.9896 & $6.330 \mathrm{E}-03$ & 2.798E-03 & $1.063 \mathrm{E}-03$ & 2.043E-04 & & & & & & & & & & & \\
\hline 6 & 0.9898 & $5.263 \mathrm{E}-03$ & 2.804E-03 & $1.368 \mathrm{E}-03$ & 5.464E-04 & 2.539E-04 & & & & & & & & & & \\
\hline 7 & 0.9906 & $4.130 \mathrm{E}-03$ & $2.540 \mathrm{E}-03$ & $1.577 \mathrm{E}-03$ & 7.818E-04 & $2.878 \mathrm{E}-04$ & 3.864E-05 & & & & & & & & & \\
\hline 8 & 0.9911 & $3.439 \mathrm{E}-03$ & 2.200E-03 & $1.537 \mathrm{E}-03$ & 9.815E-04 & 4.477E-04 & $1.590 \mathrm{E}-04$ & $1.293 \mathrm{E}-04$ & & & & & & & & \\
\hline 9 & 0.9916 & $3.101 \mathrm{E}-03$ & $1.877 \mathrm{E}-03$ & $1.450 \mathrm{E}-03$ & $1.020 \mathrm{E}-03$ & $5.880 \mathrm{E}-04$ & 2.773E-04 & 8.963E-05 & $9.946 \mathrm{E}-06$ & & & & & & & \\
\hline 10 & 0.9919 & $2.826 \mathrm{E}-03$ & $1.659 \mathrm{E}-03$ & $1.306 \mathrm{E}-03$ & $1.006 \mathrm{E}-03$ & 6.685E-04 & 3.767E-04 & $1.736 \mathrm{E}-04$ & 5.146E-05 & 5.309E-06 & & & & & & \\
\hline 11 & 0.9922 & $2.601 \mathrm{E}-03$ & $1.503 \mathrm{E}-03$ & $1.165 \mathrm{E}-03$ & 9.505E-04 & 7.024E-04 & 4.496E-04 & 2.493E-04 & $1.098 \mathrm{E}-04$ & $3.010 \mathrm{E}-05$ & $1.711 \mathrm{E}-05$ & & & & & \\
\hline 12 & 0.9925 & $2.368 \mathrm{E}-03$ & $1.399 \mathrm{E}-03$ & $1.046 \mathrm{E}-03$ & 8.748E-04 & $6.992 \mathrm{E}-04$ & 4.959E-04 & $3.102 \mathrm{E}-04$ & $1.677 \mathrm{E}-04$ & 7.006E-05 & $1.791 \mathrm{E}-05$ & $1.646 \mathrm{E}-06$ & & & & \\
\hline 13 & 0.9928 & $2.172 \mathrm{E}-03$ & $1.312 \mathrm{E}-03$ & 9.538E-04 & 7.962E-04 & 6.702E-04 & $5.168 \mathrm{E}-04$ & 3.554E-04 & 2.177E-04 & $1.139 \mathrm{E}-04$ & $4.514 \mathrm{E}-05$ & $1.083 \mathrm{E}-05$ & 9.495E-07 & & & \\
\hline 14 & 0.9931 & 2.007E-03 & $1.235 \mathrm{E}-03$ & 8.829E-04 & 7.249E-04 & $6.270 \mathrm{E}-04$ & 5.159E-04 & 3.846E-04 & 2.581E-04 & $1.544 \mathrm{E}-04$ & 7.799E-05 & $2.936 \mathrm{E}-05$ & 6.644E-06 & 5.584E-07 & & \\
\hline 15 & 0.9933 & $1.867 \mathrm{E}-03$ & $1.164 \mathrm{E}-03$ & 8.272E-04 & 6.649E-04 & 5.791E-04 & $4.988 \mathrm{E}-04$ & 3.981E-04 & 2.884E-04 & $1.892 \mathrm{E}-04$ & $1.104 \mathrm{E}-04$ & $5.378 \mathrm{E}-05$ & 1.927E-05 & 4.129E-06 & 3.337E-07 & \\
\hline 16 & 0.9936 & $1.746 \mathrm{E}-03$ & $1.099 \mathrm{E}-03$ & 7.819E-04 & 6.164E-04 & 5.331E-04 & $4.718 \mathrm{E}-04$ & 3.977E-04 & $3.081 \mathrm{E}-04$ & $2.176 \mathrm{E}-04$ & $1.397 \mathrm{E}-04$ & $7.951 \mathrm{E}-05$ & 3.733E-05 & $1.275 \mathrm{E}-05$ & $2.595 \mathrm{E}-06$ & $5.086 \mathrm{E}-06$ \\
\hline
\end{tabular}

Table 19. Estimated CCF industry-wide prior distributions with CCF events in the GC cause group from 1997 through 2015.

\begin{tabular}{|c|c|c|c|c|c|c|c|c|c|c|c|c|c|c|c|c|}
\hline $\begin{array}{c}\text { Group } \\
\text { Size }\end{array}$ & $\alpha_{1}$ & $b_{1}$ & $\alpha_{2}$ & $b_{2}$ & $\alpha_{3}$ & $b_{3}$ & $\alpha_{4}$ & $\mathrm{~b}_{4}$ & $\alpha_{5}$ & $\mathrm{~b}_{5}$ & $\alpha_{6}$ & $b_{6}$ & $\alpha_{7}$ & $\mathrm{~b}_{7}$ & $\alpha_{8}$ & $b_{8}$ \\
\hline 2 & $4.384 \mathrm{E}+01$ & $4.834 \mathrm{E}-01$ & 4.834E-01 & $4.384 \mathrm{E}+01$ & & & & & & & & & & & & \\
\hline 3 & $1.297 \mathrm{E}+02$ & $1.226 \mathrm{E}+00$ & $9.769 \mathrm{E}-01$ & $1.299 \mathrm{E}+02$ & $2.487 \mathrm{E}-01$ & $1.306 \mathrm{E}+02$ & & & & & & & & & & \\
\hline 4 & $1.819 \mathrm{E}+02$ & $2.057 \mathrm{E}+00$ & $1.442 \mathrm{E}+00$ & $1.825 \mathrm{E}+02$ & $4.114 \mathrm{E}-01$ & $1.835 \mathrm{E}+02$ & $2.036 \mathrm{E}-01$ & $1.837 \mathrm{E}+02$ & & & & & & & & \\
\hline 5 & $3.506 \mathrm{E}+02$ & $3.683 E+00$ & $2.243 \mathrm{E}+00$ & $3.521 E+02$ & 9.912E-01 & $3.533 \mathrm{E}+02$ & $3.765 \mathrm{E}-01$ & $3.539 \mathrm{E}+02$ & 7.237E-02 & $3.542 \mathrm{E}+02$ & & & & & & \\
\hline 6 & $4.003 \mathrm{E}+02$ & $4.139 \mathrm{E}+00$ & $2.128 \mathrm{E}+00$ & $4.023 \mathrm{E}+02$ & $1.134 \mathrm{E}+00$ & $4.033 \mathrm{E}+02$ & $5.533 \mathrm{E}-01$ & $4.039 \mathrm{E}+02$ & $2.210 \mathrm{E}-01$ & $4.042 \mathrm{E}+02$ & $1.027 \mathrm{E}-01$ & $4.043 \mathrm{E}+02$ & & & & \\
\hline 7 & $6.811 \mathrm{E}+02$ & $6.432 \mathrm{E}+00$ & $2.840 \mathrm{E}+00$ & $6.847 \mathrm{E}+02$ & $1.746 \mathrm{E}+00$ & $6.858 \mathrm{E}+02$ & $1.084 \mathrm{E}+00$ & $6.865 \mathrm{E}+02$ & 5.376E-01 & $6.870 \mathrm{E}+02$ & $1.979 \mathrm{E}-01$ & $6.874 \mathrm{E}+02$ & 2.656E-02 & $6.875 \mathrm{E}+02$ & & \\
\hline 8 & $6.811 \mathrm{E}+02$ & $6.112 \mathrm{E}+00$ & $2.364 \mathrm{E}+00$ & $6.848 \mathrm{E}+02$ & $1.512 \mathrm{E}+00$ & $6.857 \mathrm{E}+02$ & $1.056 \mathrm{E}+00$ & $6.861 \mathrm{E}+02$ & 6.745E-01 & $6.865 \mathrm{E}+02$ & $3.076 \mathrm{E}-01$ & $6.869 \mathrm{E}+02$ & $1.093 \mathrm{E}-01$ & $6.871 \mathrm{E}+02$ & $8.885 \mathrm{E}-02$ & $6.871 \mathrm{E}+02$ \\
\hline 9 & $1.140 \mathrm{E}+03$ & $9.669 \mathrm{E}+00$ & $3.564 \mathrm{E}+00$ & $1.146 \mathrm{E}+03$ & $2.158 \mathrm{E}+00$ & $1.147 \mathrm{E}+03$ & $1.666 \mathrm{E}+00$ & $1.148 \mathrm{E}+03$ & $1.172 \mathrm{E}+00$ & $1.148 \mathrm{E}+03$ & $6.758 \mathrm{E}-01$ & $1.149 \mathrm{E}+03$ & $3.187 \mathrm{E}-01$ & $1.149 \mathrm{E}+03$ & $1.030 \mathrm{E}-01$ & $1.149 \mathrm{E}+03$ \\
\hline
\end{tabular}




\begin{tabular}{|c|c|c|c|c|c|c|c|c|c|c|c|c|c|c|c|c|}
\hline $\begin{array}{c}\text { Group } \\
\text { Size }\end{array}$ & $\alpha_{1}$ & $b_{1}$ & $\alpha_{2}$ & $\mathrm{~b}_{2}$ & $\alpha_{3}$ & $\mathrm{~b}_{3}$ & $\alpha_{4}$ & $\mathrm{~b}_{4}$ & $\alpha_{5}$ & $b_{5}$ & $\alpha_{6}$ & $\mathrm{~b}_{6}$ & $\alpha_{7}$ & $\mathrm{~b}_{7}$ & $\alpha_{8}$ & $\mathrm{~b}_{8}$ \\
\hline 10 & $1.424 \mathrm{E}+03$ & $1.159 \mathrm{E}+01$ & $4.057 \mathrm{E}+00$ & $1.432 \mathrm{E}+03$ & $2.382 \mathrm{E}+00$ & $1.433 \mathrm{E}+03$ & $1.875 \mathrm{E}+00$ & $1.434 \mathrm{E}+03$ & $1.444 \mathrm{E}+00$ & $1.434 \mathrm{E}+03$ & $9.597 \mathrm{E}-01$ & $1.435 \mathrm{E}+03$ & 5.408E-01 & $1.435 \mathrm{E}+03$ & $2.493 \mathrm{E}-01$ & $1.435 \mathrm{E}+03$ \\
\hline 11 & $1.466 \mathrm{E}+03$ & $1.149 \mathrm{E}+01$ & $3.842 \mathrm{E}+00$ & $1.474 \mathrm{E}+03$ & $2.221 \mathrm{E}+00$ & $1.475 \mathrm{E}+03$ & $1.721 \mathrm{E}+00$ & $1.476 \mathrm{E}+03$ & $1.404 \mathrm{E}+00$ & $1.476 \mathrm{E}+03$ & $1.038 \mathrm{E}+00$ & $1.476 \mathrm{E}+03$ & 6.643E-01 & $1.477 \mathrm{E}+03$ & $3.683 \mathrm{E}-01$ & $1.477 \mathrm{E}+03$ \\
\hline 12 & $2.120 \mathrm{E}+03$ & $1.592 \mathrm{E}+01$ & $5.058 \mathrm{E}+00$ & $2.131 \mathrm{E}+03$ & $2.989 \mathrm{E}+00$ & $2.133 \mathrm{E}+03$ & $2.235 \mathrm{E}+00$ & $2.134 \mathrm{E}+03$ & $1.869 \mathrm{E}+00$ & $2.134 \mathrm{E}+03$ & $1.494 \mathrm{E}+00$ & $2.135 E+03$ & $1.060 \mathrm{E}+00$ & $2.135 \mathrm{E}+03$ & $6.626 \mathrm{E}-01$ & $2.136 \mathrm{E}+03$ \\
\hline 13 & $2.538 \mathrm{E}+03$ & $1.832 \mathrm{E}+01$ & $5.553 \mathrm{E}+00$ & $2.551 \mathrm{E}+03$ & $3.354 \mathrm{E}+00$ & $2.553 \mathrm{E}+03$ & $2.439 \mathrm{E}+00$ & $2.554 \mathrm{E}+03$ & $2.036 \mathrm{E}+00$ & $2.555 \mathrm{E}+03$ & $1.713 \mathrm{E}+00$ & $2.555 \mathrm{E}+03$ & $1.321 \mathrm{E}+00$ & $2.555 \mathrm{E}+03$ & $9.086 \mathrm{E}-01$ & $2.556 \mathrm{E}+03$ \\
\hline 14 & $3.006 \mathrm{E}+03$ & $2.090 \mathrm{E}+01$ & $6.074 \mathrm{E}+00$ & $3.021 \mathrm{E}+03$ & $3.737 \mathrm{E}+00$ & $3.023 \mathrm{E}+03$ & $2.672 \mathrm{E}+00$ & $3.024 \mathrm{E}+03$ & $2.194 \mathrm{E}+00$ & $3.025 \mathrm{E}+03$ & $1.898 \mathrm{E}+00$ & $3.025 \mathrm{E}+03$ & $1.562 \mathrm{E}+00$ & $3.025 \mathrm{E}+03$ & $1.164 \mathrm{E}+00$ & $3.026 \mathrm{E}+03$ \\
\hline 15 & $3.527 \mathrm{E}+03$ & $2.366 \mathrm{E}+01$ & $6.627 \mathrm{E}+00$ & $3.544 \mathrm{E}+03$ & $4.133 \mathrm{E}+00$ & $3.546 \mathrm{E}+03$ & $2.937 \mathrm{E}+00$ & $3.548 \mathrm{E}+03$ & $2.361 \mathrm{E}+00$ & $3.548 \mathrm{E}+03$ & $2.056 \mathrm{E}+00$ & $3.548 \mathrm{E}+03$ & $1.771 \mathrm{E}+00$ & $3.549 \mathrm{E}+03$ & $1.413 \mathrm{E}+00$ & $3.549 \mathrm{E}+03$ \\
\hline 16 & $3.310 \mathrm{E}+03$ & $2.149 \mathrm{E}+01$ & $5.818 \mathrm{E}+00$ & $3.326 \mathrm{E}+03$ & $3.662 \mathrm{E}+00$ & $3.328 \mathrm{E}+03$ & $2.605 \mathrm{E}+00$ & $3.329 \mathrm{E}+03$ & $2.054 \mathrm{E}+00$ & $3.330 \mathrm{E}+03$ & $1.776 \mathrm{E}+00$ & $3.330 \mathrm{E}+03$ & $1.572 \mathrm{E}+00$ & $3.330 \mathrm{E}+03$ & $1.325 \mathrm{E}+00$ & $3.330 \mathrm{E}+03$ \\
\hline
\end{tabular}

\begin{tabular}{|c|c|c|c|c|c|c|c|c|c|c|c|c|c|c|c|c|}
\hline $\begin{array}{c}\text { Group } \\
\text { Size }\end{array}$ & $\alpha_{9}$ & $\mathrm{~b}_{9}$ & $\alpha_{10}$ & $b_{10}$ & $\alpha_{11}$ & $\mathrm{~b}_{11}$ & $\alpha_{12}$ & $b_{12}$ & $\alpha_{13}$ & $b_{13}$ & $\alpha_{14}$ & $b_{14}$ & $\alpha_{15}$ & $b_{15}$ & $\alpha_{16}$ & $\mathrm{~b}_{16}$ \\
\hline 9 & $1.143 \mathrm{E}-02$ & $1.149 \mathrm{E}+03$ & & & & & & & & & & & & & & \\
\hline 10 & 7.388E-02 & $1.436 \mathrm{E}+03$ & 7.622E-03 & $1.436 \mathrm{E}+03$ & & & & & & & & & & & & \\
\hline 11 & $1.622 \mathrm{E}-01$ & $1.477 \mathrm{E}+03$ & 4.446E-02 & $1.477 \mathrm{E}+03$ & $2.528 \mathrm{E}-02$ & $1.477 \mathrm{E}+03$ & & & & & & & & & & \\
\hline 12 & $3.582 \mathrm{E}-01$ & $2.136 \mathrm{E}+03$ & $1.497 \mathrm{E}-01$ & $2.136 \mathrm{E}+03$ & $3.826 \mathrm{E}-02$ & $2.136 \mathrm{E}+03$ & $3.516 \mathrm{E}-03$ & $2.136 \mathrm{E}+03$ & & & & & & & & \\
\hline 13 & $5.564 \mathrm{E}-01$ & $2.556 \mathrm{E}+03$ & 2.912E-01 & $2.556 \mathrm{E}+03$ & $1.154 \mathrm{E}-01$ & $2.557 \mathrm{E}+03$ & $2.769 \mathrm{E}-02$ & $2.557 \mathrm{E}+03$ & $2.427 \mathrm{E}-03$ & $2.557 \mathrm{E}+03$ & & & & & & \\
\hline 14 & 7.811E-01 & $3.026 \mathrm{E}+03$ & $4.674 \mathrm{E}-01$ & $3.026 \mathrm{E}+03$ & $2.361 \mathrm{E}-01$ & $3.027 \mathrm{E}+03$ & $8.888 \mathrm{E}-02$ & $3.027 \mathrm{E}+03$ & $2.011 \mathrm{E}-02$ & $3.027 \mathrm{E}+03$ & $1.690 \mathrm{E}-03$ & $3.027 \mathrm{E}+03$ & & & & \\
\hline 15 & $1.024 \mathrm{E}+00$ & $3.549 \mathrm{E}+03$ & 6.717E-01 & $3.550 \mathrm{E}+03$ & 3.921E-01 & $3.550 \mathrm{E}+03$ & $1.909 \mathrm{E}-01$ & $3.550 \mathrm{E}+03$ & $6.843 \mathrm{E}-02$ & $3.550 \mathrm{E}+03$ & $1.466 \mathrm{E}-02$ & $3.551 \mathrm{E}+03$ & 1.185E-03 & $3.551 \mathrm{E}+03$ & & \\
\hline 16 & $1.026 \mathrm{E}+00$ & $3.331 \mathrm{E}+03$ & 7.249E-01 & $3.331 \mathrm{E}+03$ & 4.655E-01 & $3.331 \mathrm{E}+03$ & $2.649 \mathrm{E}-01$ & $3.331 \mathrm{E}+03$ & $1.244 \mathrm{E}-01$ & $3.331 \mathrm{E}+03$ & $4.249 \mathrm{E}-02$ & $3.332 \mathrm{E}+03$ & 8.646E-03 & $3.332 \mathrm{E}+03$ & & \\
\hline
\end{tabular}




\subsection{Prior Distributions for the "Design" Cause Group (GD)}

To estimate prior distributions for GD (i.e., Design) CCF cause group, the following selection criteria are defined on the CCF Database website:

- Type of CCF Event Level: All Level CCF Events

- CCF Event Type: CCF Events Only

- Date Range: 1997-2015

- Filter Independent Events by Selected Cause(s): True

- Shock Criteria: All Events

- Redundancy Range: Minimum = 2, Maximum $=16$

- Bayesian Update Method: Mean Method

- Failure Modes: select all failure modes except Setpoint

- CCF Categories: Cause $\rightarrow$ Design

A total of $74 \mathrm{CCF}$ events and 1,128.0 effective independent failure events related to the above selection criteria.

Additional criterion on CCF Categories $\rightarrow$ Degree $\rightarrow$ Almost/Partial or Complete is used to obtain the partial CCF events and complete CCF events, as required in the existing process. The unmapped/mapped impact vectors are also acquired from the CCF Database website. The mapped impact vectors for partial CCF events for each group size obtained from the website are used directly in the study.

Table 20 shows the number of partial CCF events, the number of complete CCF events, and the total number of CCF events for the GD cause group. The same binomial regression treatment used for complete $\mathrm{CCF}$ events was conducted. The estimated number of complete $\mathrm{CCF}$ events for each group size is listed in Table 20.

Table 21 shows the mapped impact vectors for partial CCF events for the GD cause groups sized 216 , as obtained from the CCF Database website. Table 22 shows the adjusted $n_{k}$ results for CCF events in GD cause groups sized 2-16, after adding the estimated number of complete CCF events. The MLEs or mean values of alpha factors for the GD cause groups of each size are then calculated, while the CalcPrior code is used to estimate the prior distributions for causal alpha factors pertaining to the GD cause group. Table 23 and Table 24 show the mean values and the distributions results, respectively.

Table 20. CCF data for the GD cause group from 1997 through 2015.

\begin{tabular}{|c|c|c|c|c|c|c|}
\hline $\begin{array}{c}\text { Group } \\
\text { Size }\end{array}$ & $\begin{array}{c}\text { No. Partial } \\
\text { CCF Events }\end{array}$ & $\begin{array}{c}\text { No. } \\
\text { Complete } \\
\text { CCF } \\
\text { Events }\end{array}$ & $\begin{array}{c}\text { Total No. } \\
\text { CCF Events }\end{array}$ & $\begin{array}{c}\text { Prob. of } \\
\text { Complete } \\
\text { CCF Event } \\
\text { - Data }\end{array}$ & $\begin{array}{c}\text { Prob. of } \\
\text { Complete } \\
\text { CCF Event - } \\
\text { Curve Fitting }\end{array}$ & $\begin{array}{c}\text { Estimated No. } \\
\text { of Complete } \\
\text { CCF Events }\end{array}$ \\
\hline 2 & 7 & 7 & 14 & 0.50000 & 0.44260 & 6.19640 \\
\hline 3 & 7 & 2 & 9 & 0.22222 & 0.24527 & 2.20746 \\
\hline 4 & 18 & 1 & 19 & 0.05263 & 0.13226 & 2.51302 \\
\hline 5 & 2 & 0 & 2 & 0.00000 & 0.08168 & 0.16336 \\
\hline 6 & 7 & 0 & 7 & 0.00000 & 0.06155 & 0.43088 \\
\hline 7 & 1 & 0 & 1 & 0.00000 & 0.05393 & 0.05393 \\
\hline 8 & 11 & 0 & 11 & 0.00000 & 0.05109 & 0.56198 \\
\hline
\end{tabular}




\begin{tabular}{|c|c|c|c|c|c|c|}
\hline $\begin{array}{c}\text { Group } \\
\text { Size }\end{array}$ & $\begin{array}{c}\text { No. Partial } \\
\text { CCF Events }\end{array}$ & $\begin{array}{c}\text { No. } \\
\text { Complete } \\
\text { CCF } \\
\text { Events }\end{array}$ & $\begin{array}{c}\text { Total No. } \\
\text { CCF Events }\end{array}$ & $\begin{array}{c}\text { Prob. of } \\
\text { Complete } \\
\text { CCF Event } \\
\text { - Data }\end{array}$ & $\begin{array}{c}\text { Prob. of } \\
\text { Complete } \\
\text { CCF Event - } \\
\text { Curve Fitting }\end{array}$ & $\begin{array}{c}\text { Estimated No. } \\
\text { of Complete } \\
\text { CCF Events }\end{array}$ \\
\hline 9 & 0 & 0 & 0 & NA & 0.05004 & 0.00000 \\
\hline 10 & 0 & 0 & 0 & NA & 0.04965 & 0.00000 \\
\hline 11 & 2 & 0 & 2 & 0.00000 & 0.04951 & 0.09902 \\
\hline 12 & 1 & 1 & 2 & 0.50000 & 0.04946 & 0.09892 \\
\hline 13 & 0 & 0 & 0 & NA & 0.04944 & 0.00000 \\
\hline 14 & 1 & 0 & 1 & 0.00000 & 0.04943 & 0.04943 \\
\hline 15 & 0 & 0 & 0 & NA & 0.04943 & 0.00000 \\
\hline 16 & 6 & 0 & 6 & 0.00000 & 0.04943 & 0.29658 \\
\hline Total & 63 & 11 & 74 & & & 12.67097 \\
\hline
\end{tabular}

Table 21. nk values for the partial CCF events in the GD cause group from 1997 through 2015.

\begin{tabular}{|c|c|c|c|c|c|c|c|c|c|c|c|c|c|c|c|c|}
\hline $\begin{array}{c}\text { Group } \\
\text { Size }\end{array}$ & $\mathrm{n}_{1}$ & $\mathrm{n}_{2}$ & $\mathrm{n}_{3}$ & $\mathrm{n}_{4}$ & $\mathrm{n}_{5}$ & $\mathrm{n}_{6}$ & $\mathrm{n}_{7}$ & $\mathrm{n}_{8}$ & $\mathrm{n}_{9}$ & $\mathrm{n}_{10}$ & $\mathrm{n}_{11}$ & $\mathrm{n}_{12}$ & $\mathrm{n}_{13}$ & $\mathrm{n}_{14}$ & $\mathrm{n}_{15}$ & $\mathrm{n}_{16}$ \\
\hline 2 & 30.924 & 7.492 & & & & & & & & & & & & & & \\
\hline 3 & 29.907 & 16.480 & 1.999 & & & & & & & & & & & & & \\
\hline 4 & 27.738 & 20.373 & 6.224 & 0.985 & & & & & & & & & & & & \\
\hline 5 & 28.283 & 18.667 & 10.107 & 3.404 & 0.516 & & & & & & & & & & & \\
\hline 6 & 28.558 & 17.382 & 11.932 & 5.535 & 2.072 & 0.257 & & & & & & & & & & \\
\hline 7 & 28.906 & 17.494 & 10.841 & 7.348 & 3.977 & 1.173 & 0.126 & & & & & & & & & \\
\hline 8 & 28.741 & 18.152 & 10.287 & 7.446 & 5.320 & 2.882 & 0.558 & 0.072 & & & & & & & & \\
\hline 9 & 29.056 & 17.918 & 10.391 & 7.472 & 5.706 & 3.798 & 1.968 & 0.337 & 0.042 & & & & & & & \\
\hline 10 & 29.205 & 17.784 & 10.680 & 7.285 & 5.906 & 4.375 & 2.803 & 1.342 & 0.202 & 0.0248 & & & & & & \\
\hline 11 & 29.245 & 17.640 & 11.130 & 7.102 & 5.881 & 4.740 & 3.377 & 2.110 & 0.906 & 0.1213 & 0.0152 & & & & & \\
\hline 12 & 29.171 & 17.825 & 11.134 & 7.245 & 5.652 & 4.984 & 3.742 & 2.669 & 1.591 & 0.6000 & 0.0743 & 0.0096 & & & & \\
\hline 13 & 29.033 & 17.986 & 11.172 & 7.514 & 5.356 & 5.011 & 4.126 & 2.922 & 2.200 & 1.1635 & 0.3942 & 0.0478 & 0.0063 & & & \\
\hline 14 & 28.807 & 18.184 & 11.174 & 7.836 & 5.319 & 4.480 & 4.794 & 2.954 & 2.540 & 1.7880 & 0.8132 & 0.2644 & 0.0324 & 0.0041 & & \\
\hline 15 & 28.502 & 18.429 & 11.100 & 8.218 & 5.328 & 4.404 & 4.391 & 3.646 & 2.404 & 2.3444 & 1.3564 & 0.5534 & 0.1869 & 0.0221 & 0.0027 & \\
\hline 16 & 28.123 & 18.741 & 10.914 & 8.659 & 5.388 & 4.324 & 4.122 & 3.818 & 2.876 & 1.9570 & 2.3277 & 0.8259 & 0.4211 & 0.1328 & 0.0152 & 0.0018 \\
\hline
\end{tabular}

Table 22. Adjusted nk values for CCF events in the GD cause group from 1997 through 2015.

\begin{tabular}{|c|c|c|c|c|c|c|c|c|c|c|c|c|c|c|c|c|c|c|}
\hline \begin{tabular}{|c|} 
Group \\
Size \\
\end{tabular} & $\mathrm{n}_{\mathrm{t}}$ & $\mathrm{n}_{\mathrm{I}}$ & $\mathrm{n}_{1}$ & $\mathrm{n}_{2}$ & $\mathrm{n}_{3}$ & $\mathrm{n}_{4}$ & $\mathrm{n}_{5}$ & $\mathrm{n}_{6}$ & $\mathrm{n}_{7}$ & $\mathrm{n}_{8}$ & $\mathrm{n}_{9}$ & $\mathrm{n}_{10}$ & $\mathrm{n}_{11}$ & $\mathrm{n}_{12}$ & $\mathrm{n}_{13}$ & $\mathrm{n}_{14}$ & $\mathrm{n}_{15}$ & $\mathrm{n}_{16}$ \\
\hline 2 & 429.27 & 384.66 & 30.924 & 13.689 & & & & & & & & & & & & & & \\
\hline 3 & 627.59 & 577.00 & 29.907 & 16.480 & 4.207 & & & & & & & & & & & & & \\
\hline 4 & 827.16 & 769.33 & 27.738 & 20.373 & 6.224 & 3.498 & & & & & & & & & & & & \\
\hline 5 & 1022.80 & 961.66 & 28.283 & 18.667 & 10.107 & 3.404 & 0.679 & & & & & & & & & & & \\
\hline 6 & 1220.16 & 1153.99 & 28.558 & 17.382 & 11.932 & 5.535 & 2.072 & 0.688 & & & & & & & & & & \\
\hline 7 & 1416.24 & 1346.32 & 28.906 & 17.494 & 10.841 & 7.348 & 3.977 & 1.173 & 0.180 & & & & & & & & & \\
\hline 8 & 1612.67 & 1538.65 & 28.741 & 18.152 & 10.287 & 7.446 & 5.320 & 2.882 & 0.558 & 0.634 & & & & & & & & \\
\hline
\end{tabular}




\begin{tabular}{|c|c|c|c|c|c|c|c|c|c|c|c|c|c|c|c|c|c|c|}
\hline $\begin{array}{l}\text { Group } \\
\text { Size }\end{array}$ & $\mathrm{n}_{\mathrm{t}}$ & $\mathrm{n}_{\mathrm{I}}$ & $\mathrm{n}_{1}$ & $\mathrm{n}_{2}$ & $\mathrm{n}_{3}$ & $\mathrm{n}_{4}$ & $\mathrm{n}_{5}$ & $\mathrm{n}_{6}$ & $\mathrm{n}_{7}$ & $\mathrm{n}_{8}$ & $n_{9}$ & $\mathrm{n}_{10}$ & $\mathrm{n}_{11}$ & $\mathrm{n}_{12}$ & $\mathrm{n}_{13}$ & $\mathrm{n}_{14}$ & $\mathrm{n}_{15}$ & $\mathrm{n}_{16}$ \\
\hline 9 & 1807.68 & 1730.99 & 29.056 & 17.918 & 10.391 & 7.472 & 5.706 & 3.798 & 1.968 & 0.337 & 0.042 & & & & & & & \\
\hline 10 & 2002.93 & 1923.32 & 29.205 & 17.784 & 10.680 & 7.285 & 5.906 & 4.375 & 2.803 & 1.342 & 0.202 & 0.0248 & & & & & & \\
\hline 11 & 2198.02 & 2115.65 & 29.245 & 17.640 & 11.130 & 7.102 & 5.881 & 4.740 & 3.377 & 2.110 & 0.906 & 0.121 & 0.1142 & & & & & \\
\hline 12 & 2392.78 & 2307.98 & 29.171 & 17.825 & 11.134 & 7.245 & 5.652 & 4.984 & 3.742 & 2.669 & 1.591 & 0.600 & 0.074 & 0.1086 & & & & \\
\hline 13 & 2587.24 & 2500.31 & 29.033 & 17.986 & 11.172 & 7.514 & 5.356 & 5.011 & 4.126 & 2.922 & 2.200 & 1.164 & 0.394 & 0.048 & 0.0063 & & & \\
\hline 14 & 2781.69 & 2692.65 & 28.807 & 18.184 & 11.174 & 7.836 & 5.319 & 4.480 & 4.794 & 2.954 & 2.540 & 1.788 & 0.813 & 0.264 & 0.032 & 0.0535 & & \\
\hline 15 & 2975.87 & 2884.98 & 28.502 & 18.429 & 11.100 & 8.218 & 5.328 & 4.404 & 4.391 & 3.646 & 2.404 & 2.344 & 1.356 & 0.553 & 0.187 & 0.022 & 0.0027 & \\
\hline 16 & 3170.25 & 3077.31 & 28.123 & 18.741 & 10.914 & 8.659 & 5.388 & 4.324 & 4.122 & 3.818 & 2.876 & 1.957 & 2.328 & 0.826 & 0.421 & 0.133 & 0.015 & 0.2984 \\
\hline
\end{tabular}

Table 23. Calculated alpha factor mean values for CCF events in the GD cause group from 1997 through 2015.

\begin{tabular}{|c|c|c|c|c|c|c|c|c|c|c|c|c|c|c|c|c|}
\hline $\begin{array}{c}\text { Group } \\
\text { Size }\end{array}$ & $\alpha_{1}$ & $\alpha_{2}$ & $\alpha_{3}$ & $\alpha_{4}$ & $\alpha_{5}$ & $\alpha_{6}$ & $\alpha_{7}$ & $\alpha_{8}$ & $\alpha_{9}$ & $\alpha_{10}$ & $\alpha_{11}$ & $\alpha_{12}$ & $\alpha_{13}$ & $\alpha_{14}$ & $\alpha_{15}$ & $\alpha_{16}$ \\
\hline 2 & 0.9681 & $3.189 \mathrm{E}-02$ & & & & & & & & & & & & & & \\
\hline 3 & 0.9670 & $2.626 \mathrm{E}-02$ & $6.703 \mathrm{E}-03$ & & & & & & & & & & & & & \\
\hline 4 & 0.9636 & $2.463 \mathrm{E}-02$ & $7.525 \mathrm{E}-03$ & $4.229 \mathrm{E}-03$ & & & & & & & & & & & & \\
\hline 5 & 0.9679 & $1.825 \mathrm{E}-02$ & $9.882 \mathrm{E}-03$ & 3.328E-03 & $6.641 \mathrm{E}-04$ & & & & & & & & & & & \\
\hline 6 & 0.9692 & $1.425 \mathrm{E}-02$ & $9.779 \mathrm{E}-03$ & $4.536 \mathrm{E}-03$ & $1.698 \mathrm{E}-03$ & $5.640 \mathrm{E}-04$ & & & & & & & & & & \\
\hline 7 & 0.9710 & $1.235 \mathrm{E}-02$ & 7.655E-03 & $5.188 \mathrm{E}-03$ & $2.808 \mathrm{E}-03$ & 8.283E-04 & $1.272 \mathrm{E}-04$ & & & & & & & & & \\
\hline 8 & 0.9719 & $1.126 \mathrm{E}-02$ & $6.379 \mathrm{E}-03$ & $4.617 \mathrm{E}-03$ & $3.299 \mathrm{E}-03$ & $1.787 \mathrm{E}-03$ & $3.463 \mathrm{E}-04$ & $3.933 \mathrm{E}-04$ & & & & & & & & \\
\hline 9 & 0.9737 & $9.912 \mathrm{E}-03$ & $5.748 \mathrm{E}-03$ & $4.133 \mathrm{E}-03$ & $3.156 \mathrm{E}-03$ & $2.101 \mathrm{E}-03$ & $1.089 \mathrm{E}-03$ & $1.866 \mathrm{E}-04$ & $2.317 \mathrm{E}-05$ & & & & & & & \\
\hline 10 & 0.9748 & $8.879 \mathrm{E}-03$ & $5.332 \mathrm{E}-03$ & $3.637 \mathrm{E}-03$ & $2.949 \mathrm{E}-03$ & $2.185 \mathrm{E}-03$ & $1.400 \mathrm{E}-03$ & $6.702 \mathrm{E}-04$ & $1.011 \mathrm{E}-04$ & $1.238 \mathrm{E}-05$ & & & & & & \\
\hline 11 & 0.9758 & $8.025 \mathrm{E}-03$ & $5.064 \mathrm{E}-03$ & $3.231 \mathrm{E}-03$ & $2.675 \mathrm{E}-03$ & $2.157 \mathrm{E}-03$ & $1.536 \mathrm{E}-03$ & $9.599 \mathrm{E}-04$ & $4.121 \mathrm{E}-04$ & $5.519 \mathrm{E}-05$ & $5.196 \mathrm{E}-05$ & & & & & \\
\hline 12 & 0.9768 & $7.450 \mathrm{E}-03$ & $4.653 \mathrm{E}-03$ & $3.028 \mathrm{E}-03$ & $2.362 \mathrm{E}-03$ & $2.083 \mathrm{E}-03$ & $1.564 \mathrm{E}-03$ & $1.115 \mathrm{E}-03$ & $6.650 \mathrm{E}-04$ & $2.508 \mathrm{E}-04$ & $3.106 \mathrm{E}-05$ & $4.537 \mathrm{E}-05$ & & & & \\
\hline 13 & 0.9776 & $6.952 \mathrm{E}-03$ & $4.318 \mathrm{E}-03$ & $2.904 \mathrm{E}-03$ & $2.070 \mathrm{E}-03$ & $1.937 \mathrm{E}-03$ & $1.595 \mathrm{E}-03$ & $1.129 \mathrm{E}-03$ & $8.501 \mathrm{E}-04$ & $4.497 \mathrm{E}-04$ & $1.523 \mathrm{E}-04$ & $1.849 \mathrm{E}-05$ & $2.423 \mathrm{E}-06$ & & & \\
\hline 14 & 0.9783 & $6.537 \mathrm{E}-03$ & $4.017 \mathrm{E}-03$ & $2.817 \mathrm{E}-03$ & $1.912 \mathrm{E}-03$ & $1.610 \mathrm{E}-03$ & $1.723 \mathrm{E}-03$ & $1.062 \mathrm{E}-03$ & $9.130 \mathrm{E}-04$ & $6.428 \mathrm{E}-04$ & $2.923 \mathrm{E}-04$ & $9.503 \mathrm{E}-05$ & $1.163 \mathrm{E}-05$ & $1.924 \mathrm{E}-05$ & & \\
\hline 15 & 0.9790 & 6.193E-03 & $3.730 \mathrm{E}-03$ & $2.762 \mathrm{E}-03$ & $1.790 \mathrm{E}-03$ & $1.480 \mathrm{E}-03$ & $1.476 \mathrm{E}-03$ & $1.225 \mathrm{E}-03$ & $8.079 \mathrm{E}-04$ & $7.878 \mathrm{E}-04$ & $4.558 \mathrm{E}-04$ & $1.860 \mathrm{E}-04$ & $6.281 \mathrm{E}-05$ & $7.419 \mathrm{E}-06$ & $9.043 \mathrm{E}-07$ & \\
\hline 16 & 0.9796 & $5.912 \mathrm{E}-03$ & $3.443 \mathrm{E}-03$ & 2.731E-03 & $1.700 \mathrm{E}-03$ & $1.364 \mathrm{E}-03$ & $1.300 \mathrm{E}-03$ & $1.204 \mathrm{E}-03$ & $9.071 \mathrm{E}-04$ & $6.173 \mathrm{E}-04$ & $7.342 \mathrm{E}-04$ & $2.605 \mathrm{E}-04$ & $1.328 \mathrm{E}-04$ & $4.190 \mathrm{E}-05$ & $4.784 \mathrm{E}-06$ & $9.411 \mathrm{E}-05$ \\
\hline
\end{tabular}


Table 24. Estimated CCF industry-wide prior distributions with CCF events in the GD cause group from 1997 through 2015.

\begin{tabular}{|c|c|c|c|c|c|c|c|c|c|c|c|c|c|c|c|c|}
\hline $\begin{array}{c}\text { Group } \\
\text { Size }\end{array}$ & $\alpha_{1}$ & $b_{1}$ & $\alpha_{2}$ & $\mathrm{~b}_{2}$ & $\alpha_{3}$ & $\mathrm{~b}_{3}$ & $\alpha_{4}$ & $\mathrm{~b}_{4}$ & $\alpha_{5}$ & $\mathrm{~b}_{5}$ & $\alpha_{6}$ & $\mathrm{~b}_{6}$ & $\alpha_{7}$ & $b_{7}$ & $\alpha_{8}$ & $\mathrm{~b}_{8}$ \\
\hline 2 & $1.366 \mathrm{E}+01$ & 4.498E-01 & 4.498E-01 & $1.366 \mathrm{E}+01$ & & & & & & & & & & & & \\
\hline 3 & $3.457 \mathrm{E}+01$ & $1.178 \mathrm{E}+00$ & 9.387E-01 & $3.481 \mathrm{E}+01$ & $2.396 \mathrm{E}-01$ & $3.551 \mathrm{E}+01$ & & & & & & & & & & \\
\hline 4 & $5.029 \mathrm{E}+01$ & $1.899 \mathrm{E}+00$ & $1.285 \mathrm{E}+00$ & $5.090 \mathrm{E}+01$ & $3.927 \mathrm{E}-01$ & $5.179 \mathrm{E}+01$ & 2.207E-01 & $5.197 \mathrm{E}+01$ & & & & & & & & \\
\hline 5 & $1.056 \mathrm{E}+02$ & $3.506 \mathrm{E}+00$ & $1.992 \mathrm{E}+00$ & $1.071 \mathrm{E}+02$ & $1.078 \mathrm{E}+00$ & $1.081 \mathrm{E}+02$ & 3.632E-01 & $1.088 \mathrm{E}+02$ & $7.248 \mathrm{E}-02$ & $1.091 \mathrm{E}+02$ & & & & & & \\
\hline 6 & $1.321 \mathrm{E}+02$ & $4.200 \mathrm{E}+00$ & $1.941 \mathrm{E}+00$ & $1.343 \mathrm{E}+02$ & $1.332 \mathrm{E}+00$ & $1.349 \mathrm{E}+02$ & 6.181E-01 & $1.356 \mathrm{E}+02$ & 2.313E-01 & $1.360 \mathrm{E}+02$ & $7.684 \mathrm{E}-02$ & $1.362 \mathrm{E}+02$ & & & & \\
\hline 7 & $2.087 \mathrm{E}+02$ & $6.223 \mathrm{E}+00$ & $2.654 \mathrm{E}+00$ & $2.122 \mathrm{E}+02$ & $1.645 \mathrm{E}+00$ & $2.132 \mathrm{E}+02$ & $1.115 \mathrm{E}+00$ & $2.138 \mathrm{E}+02$ & 6.034E-01 & $2.143 \mathrm{E}+02$ & $1.780 \mathrm{E}-01$ & $2.147 \mathrm{E}+02$ & 2.734E-02 & $2.149 \mathrm{E}+02$ & & \\
\hline 8 & $2.162 \mathrm{E}+02$ & $6.245 \mathrm{E}+00$ & $2.504 \mathrm{E}+00$ & $2.199 \mathrm{E}+02$ & $1.419 \mathrm{E}+00$ & $2.210 \mathrm{E}+02$ & $1.027 \mathrm{E}+00$ & $2.214 \mathrm{E}+02$ & 7.337E-01 & $2.217 \mathrm{E}+02$ & $3.975 \mathrm{E}-01$ & $2.220 \mathrm{E}+02$ & 7.703E-02 & $2.223 \mathrm{E}+02$ & 8.749E-02 & $2.223 \mathrm{E}+02$ \\
\hline 9 & $3.756 \mathrm{E}+02$ & $1.016 \mathrm{E}+01$ & $3.824 \mathrm{E}+00$ & $3.819 \mathrm{E}+02$ & $2.217 \mathrm{E}+00$ & $3.835 \mathrm{E}+02$ & $1.594 \mathrm{E}+00$ & $3.842 \mathrm{E}+02$ & $1.218 \mathrm{E}+00$ & $3.845 \mathrm{E}+02$ & $8.105 \mathrm{E}-01$ & $3.849 \mathrm{E}+02$ & 4.200E-01 & $3.853 \mathrm{E}+02$ & 7.199E-02 & $3.857 \mathrm{E}+02$ \\
\hline 10 & $4.693 \mathrm{E}+02$ & $1.212 \mathrm{E}+01$ & $4.275 \mathrm{E}+00$ & $4.772 \mathrm{E}+02$ & $2.567 \mathrm{E}+00$ & $4.789 \mathrm{E}+02$ & $1.751 \mathrm{E}+00$ & $4.797 \mathrm{E}+02$ & $1.420 \mathrm{E}+00$ & $4.800 \mathrm{E}+02$ & $1.052 \mathrm{E}+00$ & $4.804 \mathrm{E}+02$ & 6.739E-01 & $4.808 \mathrm{E}+02$ & 3.227E-01 & $4.811 \mathrm{E}+02$ \\
\hline 11 & $4.711 \mathrm{E}+02$ & $1.167 \mathrm{E}+01$ & $3.874 \mathrm{E}+00$ & $4.789 \mathrm{E}+02$ & $2.445 \mathrm{E}+00$ & $4.803 \mathrm{E}+02$ & $1.560 \mathrm{E}+00$ & $4.812 \mathrm{E}+02$ & $1.292 \mathrm{E}+00$ & $4.815 \mathrm{E}+02$ & $1.041 \mathrm{E}+00$ & $4.817 \mathrm{E}+02$ & 7.416E-01 & $4.820 \mathrm{E}+02$ & 4.634E-01 & $4.823 \mathrm{E}+02$ \\
\hline 12 & $5.588 \mathrm{E}+02$ & $1.330 \mathrm{E}+01$ & $4.262 \mathrm{E}+00$ & $5.679 \mathrm{E}+02$ & $2.662 \mathrm{E}+00$ & $5.695 \mathrm{E}+02$ & $1.732 \mathrm{E}+00$ & $5.704 \mathrm{E}+02$ & $1.351 \mathrm{E}+00$ & $5.708 \mathrm{E}+02$ & $1.192 \mathrm{E}+00$ & $5.709 \mathrm{E}+02$ & 8.946E-01 & $5.712 \mathrm{E}+02$ & 6.382E-01 & $5.715 \mathrm{E}+02$ \\
\hline 13 & $8.293 \mathrm{E}+02$ & $1.898 \mathrm{E}+01$ & $5.897 \mathrm{E}+00$ & $8.424 \mathrm{E}+02$ & $3.663 \mathrm{E}+00$ & $8.446 \mathrm{E}+02$ & $2.464 \mathrm{E}+00$ & $8.458 \mathrm{E}+02$ & $1.756 \mathrm{E}+00$ & $8.466 \mathrm{E}+02$ & $1.643 \mathrm{E}+00$ & $8.467 \mathrm{E}+02$ & $1.353 \mathrm{E}+00$ & $8.470 \mathrm{E}+02$ & 9.581E-01 & $8.474 \mathrm{E}+02$ \\
\hline 14 & $8.017 \mathrm{E}+02$ & $1.774 \mathrm{E}+01$ & $5.357 \mathrm{E}+00$ & $8.141 \mathrm{E}+02$ & $3.292 \mathrm{E}+00$ & $8.162 \mathrm{E}+02$ & $2.309 \mathrm{E}+00$ & $8.172 \mathrm{E}+02$ & $1.567 \mathrm{E}+00$ & $8.179 \mathrm{E}+02$ & $1.320 \mathrm{E}+00$ & $8.182 \mathrm{E}+02$ & $1.412 \mathrm{E}+00$ & $8.181 \mathrm{E}+02$ & 8.703E-01 & $8.186 \mathrm{E}+02$ \\
\hline 15 & $1.138 \mathrm{E}+03$ & $2.437 \mathrm{E}+01$ & $7.200 \mathrm{E}+00$ & $1.155 \mathrm{E}+03$ & $4.337 \mathrm{E}+00$ & $1.158 \mathrm{E}+03$ & $3.211 \mathrm{E}+00$ & $1.159 \mathrm{E}+03$ & $2.081 \mathrm{E}+00$ & $1.161 \mathrm{E}+03$ & $1.721 \mathrm{E}+00$ & $1.161 \mathrm{E}+03$ & $1.716 \mathrm{E}+00$ & $1.161 \mathrm{E}+03$ & $1.425 \mathrm{E}+00$ & $1.161 \mathrm{E}+03$ \\
\hline 16 & $9.389 \mathrm{E}+02$ & $1.960 \mathrm{E}+01$ & $5.667 \mathrm{E}+00$ & $9.529 \mathrm{E}+02$ & $3.300 \mathrm{E}+00$ & $9.552 \mathrm{E}+02$ & $2.618 \mathrm{E}+00$ & $9.559 \mathrm{E}+02$ & $1.629 \mathrm{E}+00$ & $9.569 \mathrm{E}+02$ & $1.308 \mathrm{E}+00$ & $9.572 \mathrm{E}+02$ & $1.246 \mathrm{E}+00$ & $9.573 \mathrm{E}+02$ & $1.154 \mathrm{E}+00$ & $9.574 \mathrm{E}+02$ \\
\hline
\end{tabular}

\begin{tabular}{|c|c|c|c|c|c|c|c|c|c|c|c|c|c|c|c|c|}
\hline $\begin{array}{c}\text { Group } \\
\text { Size }\end{array}$ & $\alpha_{9}$ & $\mathrm{~b}_{9}$ & $\alpha_{10}$ & $\mathrm{~b}_{10}$ & $\alpha_{11}$ & $b_{11}$ & $\alpha_{12}$ & $b_{12}$ & $\alpha_{13}$ & $b_{13}$ & $\alpha_{14}$ & $\mathrm{~b}_{14}$ & $\alpha_{15}$ & $b_{15}$ & $\alpha_{16}$ & $\mathrm{~b}_{16}$ \\
\hline 9 & 8.938E-03 & $3.857 \mathrm{E}+02$ & & & & & & & & & & & & & & \\
\hline 10 & $4.866 \mathrm{E}-02$ & $4.814 \mathrm{E}+02$ & 5.959E-03 & $4.815 \mathrm{E}+02$ & & & & & & & & & & & & \\
\hline 11 & $1.989 \mathrm{E}-01$ & $4.825 \mathrm{E}+02$ & $2.664 \mathrm{E}-02$ & $4.827 \mathrm{E}+02$ & $2.509 \mathrm{E}-02$ & $4.827 \mathrm{E}+02$ & & & & & & & & & & \\
\hline 12 & 3.805E-01 & $5.717 \mathrm{E}+02$ & $1.435 \mathrm{E}-01$ & $5.720 \mathrm{E}+02$ & $1.777 \mathrm{E}-02$ & $5.721 \mathrm{E}+02$ & $2.596 \mathrm{E}-02$ & $5.721 \mathrm{E}+02$ & & & & & & & & \\
\hline 13 & 7.212E-01 & $8.476 \mathrm{E}+02$ & $3.815 \mathrm{E}-01$ & $8.479 \mathrm{E}+02$ & $1.292 \mathrm{E}-01$ & $8.482 \mathrm{E}+02$ & $1.569 \mathrm{E}-02$ & $8.483 \mathrm{E}+02$ & $2.056 \mathrm{E}-03$ & $8.483 \mathrm{E}+02$ & & & & & & \\
\hline 14 & 7.482E-01 & $8.187 \mathrm{E}+02$ & $5.268 \mathrm{E}-01$ & $8.189 \mathrm{E}+02$ & $2.396 \mathrm{E}-01$ & $8.192 \mathrm{E}+02$ & 7.788E-02 & $8.194 \mathrm{E}+02$ & $9.530 \mathrm{E}-03$ & $8.195 \mathrm{E}+02$ & $1.577 \mathrm{E}-02$ & $8.195 \mathrm{E}+02$ & & & & \\
\hline 15 & 9.393E-01 & $1.162 \mathrm{E}+03$ & $9.159 \mathrm{E}-01$ & $1.162 \mathrm{E}+03$ & $5.299 \mathrm{E}-01$ & $1.162 \mathrm{E}+03$ & $2.162 \mathrm{E}-01$ & $1.162 \mathrm{E}+03$ & 7.302E-02 & $1.163 \mathrm{E}+03$ & $8.625 \mathrm{E}-03$ & $1.163 \mathrm{E}+03$ & $1.051 \mathrm{E}-03$ & $1.163 \mathrm{E}+03$ & & \\
\hline 16 & $8.695 \mathrm{E}-01$ & $9.577 \mathrm{E}+02$ & 5.917E-01 & $9.579 \mathrm{E}+02$ & 7.038E-01 & $9.578 \mathrm{E}+02$ & $2.497 \mathrm{E}-01$ & $9.583 \mathrm{E}+02$ & $1.273 \mathrm{E}-01$ & $9.584 \mathrm{E}+02$ & $4.016 \mathrm{E}-02$ & $9.585 \mathrm{E}+02$ & 4.586E-03 & $9.585 \mathrm{E}+02$ & $9.021 \mathrm{E}-02$ & $9.584 \mathrm{E}+02$ \\
\hline
\end{tabular}




\subsection{Prior Distributions for the "Environment" Cause Group (GE)}

To estimate prior distributions for the GE (i.e., Environment) CCF cause group, the following selection criteria are defined in the CCF Database website:

- Type of CCF Event Level: All Level CCF Events

- CCF Event Type: CCF Events Only

- Date Range: 1997-2015

- Filter Independent Events by Selected Cause(s): True

- Shock Criteria: All Events

- Redundancy Range: Minimum = 2, Maximum $=16$

- Bayesian Update Method: Mean Method

- Failure Modes: select all failure modes except Setpoint

- CCF Categories: Cause $\rightarrow$ Environment

A total of $57 \mathrm{CCF}$ events and 467.9 effective independent failure events related to the above selection criteria.

Additional criterion on CCF Categories $\rightarrow$ Degree $\rightarrow$ Almost/Partial or Complete is used to obtain the partial CCF events and complete CCF events, as required in the existing process. The unmapped/mapped impact vectors are also acquired from the CCF Database website. The mapped impact vectors for partial CCF events for each group size obtained from the website are used directly in the study.

Table 25 shows the number of partial CCF events, the number of complete CCF events, and the total number of $\mathrm{CCF}$ events for the GE cause group. The same binomial regression treatment used for complete $\mathrm{CCF}$ events was conducted. The estimated number of complete $\mathrm{CCF}$ events for each group size is listed in Table $25^{\mathrm{b}}$.

Table 26 shows the mapped impact vectors for partial CCF events for the GE cause groups sized 216, as obtained from the CCF Database website. Table 27 shows the adjusted $n_{k}$ results for CCF events in the GE cause groups sized 2-16, after adding the estimated number of complete CCF events. The MLEs or mean values of alpha factors for the GE cause groups of each size are then calculated, while the CalcPrior code is used to estimate the prior distributions for causal alpha factors pertaining to the GE cause group. Table 28 and Table 29 show the mean values and the distributions results, respectively.

b Note that the curve-fitting parameter value calculated by MatLab using Eq. 5 would lead to negative values for higher group sizes. Thus, the curve parameter value was adjusted manually to avoid negative values for the probability of complete CCF events. 
Table 25. CCF data for the GE cause group from 1997 through 2015.

\begin{tabular}{|c|c|c|c|c|c|c|}
\hline $\begin{array}{c}\text { Group } \\
\text { Size }\end{array}$ & $\begin{array}{c}\text { Portial } \\
\text { CCF } \\
\text { Events }\end{array}$ & $\begin{array}{c}\text { No. } \\
\text { Complete } \\
\text { CCF } \\
\text { Events }\end{array}$ & $\begin{array}{c}\text { Total No. } \\
\text { CCF } \\
\text { Events }\end{array}$ & $\begin{array}{c}\text { Prob. of } \\
\text { Complete } \\
\text { CCF } \\
\text { Event - } \\
\text { Data }\end{array}$ & $\begin{array}{c}\text { Prob. of } \\
\text { Complete } \\
\text { CCF Event - } \\
\text { Curve } \\
\text { Fitting }\end{array}$ & $\begin{array}{c}\text { Estimated } \\
\text { No. of } \\
\text { Complete } \\
\text { CCF Events }\end{array}$ \\
\hline 2 & 2 & 11 & 13 & 0.84615 & 0.72767 & 9.45974 \\
\hline 3 & 5 & 6 & 11 & 0.54545 & 0.49731 & 5.47045 \\
\hline 4 & 15 & 1 & 16 & 0.06250 & 0.27022 & 4.32346 \\
\hline 5 & 2 & 0 & 2 & 0.00000 & 0.12475 & 0.24951 \\
\hline 6 & 9 & 0 & 9 & 0.00000 & 0.05550 & 0.49947 \\
\hline 7 & 1 & 0 & 1 & 0.00000 & 0.02718 & 0.02718 \\
\hline 8 & 0 & 0 & 0 & NA & 0.01633 & 0.00000 \\
\hline 9 & 0 & 0 & 0 & NA & 0.01227 & 0.00000 \\
\hline 10 & 0 & 0 & 0 & NA & 0.01078 & 0.00000 \\
\hline 11 & 0 & 0 & 0 & NA & 0.01022 & 0.00000 \\
\hline 12 & 5 & 0 & 5 & 0.00000 & 0.01002 & 0.05010 \\
\hline 13 & 0 & 0 & 0 & NA & 0.00994 & 0.00000 \\
\hline 14 & 0 & 0 & 0 & NA & 0.00992 & 0.00000 \\
\hline 15 & 0 & 0 & 0 & NA & 0.00991 & 0.00000 \\
\hline 16 & 0 & 0 & 0 & NA & 0.00990 & 0.00000 \\
\hline Total & 39 & 18 & 57 & & & 20.07989 \\
\hline
\end{tabular}

Table 26. nk values for the partial CCF events in the GE cause group from 1997 through 2015.

\begin{tabular}{|c|c|c|c|c|c|c|c|c|c|c|c|c|c|c|c|c|}
\hline $\begin{array}{c}\text { Group } \\
\text { Size }\end{array}$ & $\mathrm{n}_{1}$ & $\mathrm{n}_{2}$ & $\mathrm{n}_{3}$ & $\mathrm{n}_{4}$ & $\mathrm{n}_{5}$ & $\mathrm{n}_{6}$ & $\mathrm{n}_{7}$ & $\mathrm{n}_{8}$ & $\mathrm{n}_{9}$ & $\mathrm{n}_{10}$ & $\mathrm{n}_{11}$ & $\mathrm{n}_{12}$ & $\mathrm{n}_{13}$ & $\mathrm{n}_{14}$ & $\mathrm{n}_{15}$ & $\mathrm{n}_{16}$ \\
\hline 2 & 23.211 & 8.115 & & & & & & & & & & & & & & \\
\hline 3 & 17.219 & 17.597 & 2.249 & & & & & & & & & & & & & \\
\hline 4 & 9.728 & 21.221 & 7.941 & 0.608 & & & & & & & & & & & & \\
\hline 5 & 7.219 & 17.451 & 11.757 & 4.228 & 0.286 & & & & & & & & & & & \\
\hline 6 & 6.310 & 12.373 & 14.186 & 6.664 & 2.286 & 0.175 & & & & & & & & & & \\
\hline 7 & 5.790 & 9.950 & 12.775 & 8.263 & 4.440 & 1.495 & 0.108 & & & & & & & & & \\
\hline 8 & 5.204 & 8.635 & 10.833 & 9.116 & 5.515 & 3.114 & 0.986 & 0.068 & & & & & & & & \\
\hline 9 & 4.562 & 7.792 & 9.217 & 9.160 & 6.296 & 4.025 & 2.229 & 0.655 & 0.043 & & & & & & & \\
\hline 10 & 3.863 & 7.303 & 7.835 & 8.822 & 6.781 & 4.611 & 3.077 & 1.608 & 0.437 & 0.0273 & & & & & & \\
\hline 11 & 3.097 & 7.117 & 6.580 & 8.367 & 6.965 & 5.076 & 3.573 & 2.398 & 1.162 & 0.2925 & 0.0175 & & & & & \\
\hline 12 & 2.243 & 7.230 & 5.347 & 7.952 & 6.894 & 5.439 & 3.923 & 2.882 & 1.875 & 0.8397 & 0.1964 & 0.0113 & & & & \\
\hline 13 & 2.086 & 6.419 & 4.969 & 6.954 & 6.912 & 5.672 & 4.234 & 3.171 & 2.370 & 1.4618 & 0.6056 & 0.1321 & 0.0073 & & & \\
\hline 14 & 1.956 & 5.723 & 4.649 & 6.119 & 6.666 & 5.835 & 4.516 & 3.394 & 2.655 & 1.9578 & 1.1329 & 0.4359 & 0.0890 & 0.0047 & & \\
\hline 15 & 1.849 & 5.120 & 4.370 & 5.427 & 6.278 & 5.879 & 4.764 & 3.615 & 2.828 & 2.2662 & 1.6114 & 0.8724 & 0.3131 & 0.0600 & 0.0031 & \\
\hline 16 & 1.763 & 4.596 & 4.119 & 4.857 & 5.827 & 5.802 & 4.949 & 3.845 & 2.977 & 2.4338 & 1.9458 & 1.3170 & 0.6676 & 0.2244 & 0.0406 & 0.0020 \\
\hline
\end{tabular}


Table 27. Adjusted nk values for CCF events in the GE cause group from 1997 through 2015.

\begin{tabular}{|c|c|c|c|c|c|c|c|c|c|c|c|c|c|c|c|c|c|c|}
\hline \begin{tabular}{|c|} 
Group \\
Size \\
\end{tabular} & $\mathrm{n}_{\mathrm{t}}$ & $\mathrm{n}_{\mathrm{I}}$ & $\mathrm{n}_{1}$ & $\mathrm{n}_{2}$ & $\mathrm{n}_{3}$ & $\mathrm{n}_{4}$ & $\mathrm{n}_{5}$ & $\mathrm{n}_{6}$ & $\mathrm{n}_{7}$ & $\mathrm{n}_{8}$ & $\mathrm{n}_{9}$ & $\mathrm{n}_{10}$ & $\mathrm{n}_{11}$ & $\mathrm{n}_{12}$ & $\mathrm{n}_{13}$ & $\mathrm{n}_{14}$ & $\mathrm{n}_{15}$ & $\mathrm{n}_{16}$ \\
\hline 2 & 250.79 & 210.00 & 23.211 & 17.574 & & & & & & & & & & & & & & \\
\hline 3 & 357.54 & 315.00 & 17.219 & 17.597 & 7.719 & & & & & & & & & & & & & \\
\hline 4 & 463.82 & 420.00 & 9.728 & 21.221 & 7.941 & 4.931 & & & & & & & & & & & & \\
\hline 5 & 566.20 & 525.01 & 7.219 & 17.451 & 11.757 & 4.228 & 0.535 & & & & & & & & & & & \\
\hline 6 & 672.50 & 630.01 & 6.310 & 12.373 & 14.186 & 6.664 & 2.286 & 0.674 & & & & & & & & & & \\
\hline 7 & 777.86 & 735.01 & 5.790 & 9.950 & 12.775 & 8.263 & 4.440 & 1.495 & 0.136 & & & & & & & & & \\
\hline 8 & 883.48 & 840.01 & 5.204 & 8.635 & 10.833 & 9.116 & 5.515 & 3.114 & 0.986 & 0.068 & & & & & & & & \\
\hline 9 & 988.99 & 945.01 & 4.562 & 7.792 & 9.217 & 9.160 & 6.296 & 4.025 & 2.229 & 0.655 & 0.043 & & & & & & & \\
\hline 10 & 1094.37 & 1050.01 & 3.863 & 7.303 & 7.835 & 8.822 & 6.781 & 4.611 & 3.077 & 1.608 & 0.437 & 0.0273 & & & & & & \\
\hline 11 & 1199.66 & 1155.01 & 3.097 & 7.117 & 6.580 & 8.367 & 6.965 & 5.076 & 3.573 & 2.398 & 1.162 & 0.293 & 0.0175 & & & & & \\
\hline 12 & 1304.89 & 1260.01 & 2.243 & 7.230 & 5.347 & 7.952 & 6.894 & 5.439 & 3.923 & 2.882 & 1.875 & 0.840 & 0.196 & 0.0614 & & & & \\
\hline 13 & 1410.01 & 1365.02 & 2.086 & 6.419 & 4.969 & 6.954 & 6.912 & 5.672 & 4.234 & 3.171 & 2.370 & 1.462 & 0.606 & 0.132 & 0.0073 & & & \\
\hline 14 & 1515.15 & 1470.02 & 1.956 & 5.723 & 4.649 & 6.119 & 6.666 & 5.835 & 4.516 & 3.394 & 2.655 & 1.958 & 1.133 & 0.436 & 0.089 & 0.0047 & & \\
\hline 15 & 1620.28 & 1575.02 & 1.849 & 5.120 & 4.370 & 5.427 & 6.278 & 5.879 & 4.764 & 3.615 & 2.828 & 2.266 & 1.611 & 0.872 & 0.313 & 0.060 & 0.0031 & \\
\hline 16 & 1725.39 & 1680.02 & 1.763 & 4.596 & 4.119 & 4.857 & 5.827 & 5.802 & 4.949 & 3.845 & 2.977 & 2.434 & 1.946 & 1.317 & 0.668 & 0.224 & 0.041 & 0.0020 \\
\hline
\end{tabular}


Table 28. Calculated alpha factor mean values for CCF events in the GE cause group from 1997 through 2015.

\begin{tabular}{|c|c|c|c|c|c|c|c|c|c|c|c|c|c|c|c|c|}
\hline $\begin{array}{c}\text { Group } \\
\text { Size }\end{array}$ & $\alpha_{1}$ & $\mathrm{~b}_{1}$ & $\alpha_{2}$ & $\mathrm{~b}_{2}$ & $\alpha_{3}$ & $\mathrm{~b}_{3}$ & $\alpha_{4}$ & $\mathrm{~b}_{4}$ & $\alpha_{5}$ & $\mathrm{~b}_{5}$ & $\alpha_{6}$ & $\mathrm{~b}_{6}$ & $\alpha_{7}$ & $b_{7}$ & $\alpha_{8}$ & $\mathrm{~b}_{8}$ \\
\hline 2 & 0.9299 & 7.008E-02 & & & & & & & & & & & & & & \\
\hline 3 & 0.9292 & $4.922 \mathrm{E}-02$ & $2.159 \mathrm{E}-02$ & & & & & & & & & & & & & \\
\hline 4 & 0.9265 & $4.575 \mathrm{E}-02$ & $1.712 \mathrm{E}-02$ & $1.063 \mathrm{E}-02$ & & & & & & & & & & & & \\
\hline 5 & 0.9400 & $3.082 \mathrm{E}-02$ & $2.076 \mathrm{E}-02$ & 7.468E-03 & 9.452E-04 & & & & & & & & & & & \\
\hline 6 & 0.9462 & $1.840 \mathrm{E}-02$ & 2.109E-02 & 9.909E-03 & $3.399 \mathrm{E}-03$ & 1.003E-03 & & & & & & & & & & \\
\hline 7 & 0.9524 & $1.279 \mathrm{E}-02$ & $1.642 \mathrm{E}-02$ & $1.062 \mathrm{E}-02$ & $5.708 \mathrm{E}-03$ & $1.921 \mathrm{E}-03$ & $1.744 \mathrm{E}-04$ & & & & & & & & & \\
\hline 8 & 0.9567 & $9.774 \mathrm{E}-03$ & $1.226 \mathrm{E}-02$ & $1.032 \mathrm{E}-02$ & $6.242 \mathrm{E}-03$ & $3.524 \mathrm{E}-03$ & $1.117 \mathrm{E}-03$ & $7.686 \mathrm{E}-05$ & & & & & & & & \\
\hline 9 & 0.9601 & $7.878 \mathrm{E}-03$ & $9.320 \mathrm{E}-03$ & $9.262 \mathrm{E}-03$ & $6.366 \mathrm{E}-03$ & $4.070 \mathrm{E}-03$ & $2.254 \mathrm{E}-03$ & $6.624 \mathrm{E}-04$ & 4.337E-05 & & & & & & & \\
\hline 10 & 0.9630 & $6.673 \mathrm{E}-03$ & 7.159E-03 & $8.061 \mathrm{E}-03$ & $6.196 \mathrm{E}-03$ & 4.213E-03 & $2.812 \mathrm{E}-03$ & $1.470 \mathrm{E}-03$ & 3.993E-04 & 2.494E-05 & & & & & & \\
\hline 11 & 0.9654 & $5.933 \mathrm{E}-03$ & $5.485 \mathrm{E}-03$ & $6.974 \mathrm{E}-03$ & $5.806 \mathrm{E}-03$ & $4.231 \mathrm{E}-03$ & $2.978 \mathrm{E}-03$ & $1.999 \mathrm{E}-03$ & 9.689E-04 & $2.438 \mathrm{E}-04$ & $1.457 \mathrm{E}-05$ & & & & & \\
\hline 12 & 0.9673 & $5.541 \mathrm{E}-03$ & 4.098E-03 & $6.094 \mathrm{E}-03$ & $5.283 \mathrm{E}-03$ & 4.168E-03 & $3.006 \mathrm{E}-03$ & $2.209 \mathrm{E}-03$ & $1.437 \mathrm{E}-03$ & $6.435 \mathrm{E}-04$ & $1.505 \mathrm{E}-04$ & 4.702E-05 & & & & \\
\hline 13 & 0.9696 & $4.552 \mathrm{E}-03$ & $3.524 \mathrm{E}-03$ & 4.932E-03 & $4.902 \mathrm{E}-03$ & 4.023E-03 & $3.003 \mathrm{E}-03$ & $2.249 \mathrm{E}-03$ & $1.681 \mathrm{E}-03$ & $1.037 \mathrm{E}-03$ & $4.295 \mathrm{E}-04$ & 9.367E-05 & 5.166E-06 & & & \\
\hline 14 & 0.9715 & $3.777 \mathrm{E}-03$ & $3.068 \mathrm{E}-03$ & $4.038 \mathrm{E}-03$ & $4.400 \mathrm{E}-03$ & $3.851 \mathrm{E}-03$ & $2.981 \mathrm{E}-03$ & $2.240 \mathrm{E}-03$ & $1.752 \mathrm{E}-03$ & $1.292 \mathrm{E}-03$ & 7.477E-04 & $2.877 \mathrm{E}-04$ & 5.874E-05 & 3.123E-06 & & \\
\hline 15 & 0.9732 & $3.160 \mathrm{E}-03$ & $2.697 \mathrm{E}-03$ & 3.349E-03 & $3.875 \mathrm{E}-03$ & $3.628 \mathrm{E}-03$ & $2.940 \mathrm{E}-03$ & $2.231 \mathrm{E}-03$ & $1.746 \mathrm{E}-03$ & $1.399 \mathrm{E}-03$ & 9.945E-04 & 5.384E-04 & $1.932 \mathrm{E}-04$ & $3.706 \mathrm{E}-05$ & $1.903 \mathrm{E}-06$ & \\
\hline 16 & 0.9747 & $2.664 \mathrm{E}-03$ & 2.387E-03 & $2.815 \mathrm{E}-03$ & $3.377 \mathrm{E}-03$ & 3.363E-03 & $2.869 \mathrm{E}-03$ & $2.229 \mathrm{E}-03$ & $1.725 \mathrm{E}-03$ & 1.411E-03 & $1.128 \mathrm{E}-03$ & 7.633E-04 & 3.870E-04 & $1.301 \mathrm{E}-04$ & $2.350 \mathrm{E}-05$ & $1.168 \mathrm{E}-06$ \\
\hline
\end{tabular}

Table 29. Estimated CCF industry-wide prior distributions with CCF events in the GE cause group from 1997 through 2015.

\begin{tabular}{|c|c|c|c|c|c|c|c|c|c|c|c|c|c|c|c|c|}
\hline $\begin{array}{l}\text { Group } \\
\text { Size }\end{array}$ & $\alpha_{1}$ & $b_{1}$ & $\alpha_{2}$ & $\mathrm{~b}_{2}$ & $\alpha_{3}$ & $\mathrm{~b}_{3}$ & $\alpha_{4}$ & $\mathrm{~b}_{4}$ & $\alpha_{5}$ & $b_{5}$ & $\alpha_{6}$ & $b_{6}$ & $\alpha_{7}$ & $\mathrm{~b}_{7}$ & $\alpha_{8}$ & $\mathrm{~b}_{8}$ \\
\hline 2 & $5.044 \mathrm{E}+00$ & $3.801 \mathrm{E}-01$ & $3.801 \mathrm{E}-01$ & $5.044 \mathrm{E}+00$ & & & & & & & & & & & & \\
\hline 3 & $1.260 \mathrm{E}+01$ & $9.601 \mathrm{E}-01$ & 6.673E-01 & $1.289 \mathrm{E}+01 \mid$ & $2.927 \mathrm{E}-01$ & $1.327 \mathrm{E}+01$ & & & & & & & & & & \\
\hline 4 & $2.103 \mathrm{E}+01$ & $1.669 \mathrm{E}+00$ & $1.039 \mathrm{E}+00$ & $2.166 \mathrm{E}+01 \mid$ & 3.887E-01 & $|2.231 \mathrm{E}+01|$ & $2.414 \mathrm{E}-01$ & $2.246 \mathrm{E}+01 \mid$ & & & & & & & & \\
\hline 5 & $5.463 \mathrm{E}+01$ & $3.487 \mathrm{E}+00$ & $1.791 \mathrm{E}+00$ & $|5.632 \mathrm{E}+01|$ & $|1.207 \mathrm{E}+00|$ & $|5.691 \mathrm{E}+01|$ & $4.340 \mathrm{E}-01$ & $|5.768 \mathrm{E}+01|$ & 5.493E-02 & $5.806 \mathrm{E}+01$ & & & & & & \\
\hline 6 & $6.867 \mathrm{E}+01$ & $3.905 \mathrm{E}+00$ & $1.335 \mathrm{E}+00$ & $7.124 \mathrm{E}+01$ & $1.531 \mathrm{E}+00$ & $7.104 \mathrm{E}+01$ & 7.191E-01 & 7.186E+01 & $2.467 \mathrm{E}-01$ & $7.233 \mathrm{E}+01$ & 7.279E-02 & $7.250 \mathrm{E}+01$ & & & & \\
\hline 7 & $1.154 \mathrm{E}+02$ & $5.770 \mathrm{E}+00$ & $1.549 \mathrm{E}+00$ & $1.196 \mathrm{E}+02$ & $|1.989 \mathrm{E}+00|$ & $|1.191 \mathrm{E}+02|$ & $1.287 \mathrm{E}+00$ & $|1.198 \mathrm{E}+02|$ & $6.913 \mathrm{E}-01$ & $|1.204 \mathrm{E}+02|$ & 2.327E-01 & $1.209 \mathrm{E}+02$ & $2.112 \mathrm{E}-02$ & $1.211 \mathrm{E}+02$ & & \\
\hline 8 & $1.550 \mathrm{E}+02$ & 7.017E+00 & $1.583 \mathrm{E}+00$ & $1.604 \mathrm{E}+02$ & $1.986 \mathrm{E}+00$ & $1.600 \mathrm{E}+02$ & $1.672 \mathrm{E}+00$ & $1.603 \mathrm{E}+02$ & $1.011 \mathrm{E}+00$ & $1.610 \mathrm{E}+02$ & 5.709E-01 & $1.614 \mathrm{E}+02$ & $1.809 \mathrm{E}-01$ & $1.618 \mathrm{E}+02$ & $1.245 \mathrm{E}-02$ & $1.620 \mathrm{E}+02$ \\
\hline 9 & $1.961 \mathrm{E}+02$ & $8.141 \mathrm{E}+00$ & $1.609 \mathrm{E}+00$ & $2.027 \mathrm{E}+02$ & $1.904 \mathrm{E}+00$ & $2.024 \mathrm{E}+02$ & $1.892 \mathrm{E}+00$ & $2.024 \mathrm{E}+02$ & $1.300 \mathrm{E}+00$ & $2.030 \mathrm{E}+02$ & $8.313 \mathrm{E}-01$ & $2.035 \mathrm{E}+02$ & 4.604E-01 & $2.038 \mathrm{E}+02$ & 1.353E-01 & $2.041 \mathrm{E}+02$ \\
\hline 10 & $2.435 \mathrm{E}+02$ & $9.356 \mathrm{E}+00$ & $1.687 \mathrm{E}+00$ & $2.511 \mathrm{E}+02$ & $\mid 1.810 \mathrm{E}+00$ & $|2.510 \mathrm{E}+02|$ & $2.038 \mathrm{E}+00$ & $|2.508 \mathrm{E}+02|$ & $1.567 \mathrm{E}+00$ & $2.513 \mathrm{E}+02 \mid$ & $1.065 \mathrm{E}+00$ & $2.518 \mathrm{E}+02 \mid$ & 7.108E-01 | & $|2.521 \mathrm{E}+02|$ & 3.715E-01 & $2.524 \mathrm{E}+\mathrm{C}$ \\
\hline
\end{tabular}




\begin{tabular}{|c|c|c|c|c|c|c|c|c|c|c|c|c|c|c|c|c|}
\hline $\begin{array}{c}\text { Group } \\
\text { Size }\end{array}$ & $\alpha_{1}$ & $\mathrm{~b}_{1}$ & $\alpha_{2}$ & $b_{2}$ & $\alpha_{3}$ & $\mathrm{~b}_{3}$ & $\alpha_{4}$ & $\mathrm{~b}_{4}$ & $\alpha_{5}$ & $\mathrm{~b}_{5}$ & $\alpha_{6}$ & $\mathrm{~b}_{6}$ & $\alpha_{7}$ & $b_{7}$ & $\alpha_{8}$ & $\mathrm{~b}_{8}$ \\
\hline 11 & $.974 \mathrm{E}+02$ & $1.067 \mathrm{E}+01$ & $1.828 \mathrm{E}+00$ & $3.062 \mathrm{E}+02$ & $1.690 \mathrm{E}+00$ & $3.064 \mathrm{E}+02$ & $2.148 \mathrm{E}+00$ & $3.059 \mathrm{E}+02$ & $1.789 \mathrm{E}+00$ & $3.063 \mathrm{E}+02$ & $1.303 \mathrm{E}+00$ & $3.068 \mathrm{E}+02$ & 9.174E-01 & $3.071 \mathrm{E}+02$ & 6.157E-01 & $3.074 \mathrm{E}+02$ \\
\hline 12 & $3.074 \mathrm{E}+02$ & $1.039 \mathrm{E}+01$ & $1.761 \mathrm{E}+00$ & $3.161 \mathrm{E}+02$ & $1.302 \mathrm{E}+00$ & $3.165 \mathrm{E}+02$ & $1.937 \mathrm{E}+00$ & $3.159 \mathrm{E}+02$ & $1.679 \mathrm{E}+00$ & $3.162 \mathrm{E}+02$ & $1.325 \mathrm{E}+00$ & $3.165 \mathrm{E}+02$ & $9.554 \mathrm{E}-01$ & $3.169 \mathrm{E}+02$ & -01 & $3.171 \mathrm{E}+02$ \\
\hline 13 & $4.303 \mathrm{E}+02$ & $1.351 \mathrm{E}+01$ & $2.020 \mathrm{E}+00$ & $4.418 \mathrm{E}+02$ & $1.564 \mathrm{E}+00$ & $4.422 \mathrm{E}+02$ & $2.189 \mathrm{E}+00$ & $4.416 \mathrm{E}+02$ & $2.175 \mathrm{E}+00$ & $4.416 \mathrm{E}+02$ & $1.785 \mathrm{E}+00$ & $4.420 \mathrm{E}+02$ & $1.333 \mathrm{E}+00$ & $4.425 \mathrm{E}+02$ & 9.979E-01 & $4.428 \mathrm{E}+02$ \\
\hline 14 & $5.112 \mathrm{E}+02$ & $1.499 \mathrm{E}+01$ & $1.987 \mathrm{E}+00$ & $5.242 \mathrm{E}+02$ & $1.615 \mathrm{E}+00$ & $5.246 \mathrm{E}+02$ & $2.125 \mathrm{E}+00$ & $5.240 \mathrm{E}+02$ & $2.315 \mathrm{E}+00$ & $5.239 \mathrm{E}+02$ & $2.026 \mathrm{E}+00$ & $5.241 \mathrm{E}+02$ & $1.568 \mathrm{E}+00$ & $5.246 \mathrm{E}+02$ & $1.179 \mathrm{E}+00$ & $5.250 \mathrm{E}+02$ \\
\hline 15 & $6.020 \mathrm{E}+02$ & $1.657 \mathrm{E}+01$ & $1.955 \mathrm{E}+00$ & $6.166 \mathrm{E}+02$ & $1.668 \mathrm{E}+00$ & $6.169 \mathrm{E}+02$ & $2.072 \mathrm{E}+00$ & $6.165 \mathrm{E}+02$ & $2.397 \mathrm{E}+00$ & $6.162 \mathrm{E}+02$ & $2.244 \mathrm{E}+00$ & $6.163 \mathrm{E}+02$ & $1.819 \mathrm{E}+00$ & $6.168 \mathrm{E}+02$ & $1.380 \mathrm{E}+00$ & $6.172 \mathrm{E}+02$ \\
\hline 16 & $7.036 \mathrm{E}+02$ & $1.824 \mathrm{E}+01$ & $1.923 \mathrm{E}+00$ & $7.199 \mathrm{E}+02$ & $1.723 \mathrm{E}+00$ & $7.201 \mathrm{E}+02$ & $2.032 \mathrm{E}+00$ & $7.198 \mathrm{E}+02$ & $2.438 \mathrm{E}+00$ & $7.194 \mathrm{E}+02$ & $2.427 \mathrm{E}+00$ & $7.194 \mathrm{E}+02$ & $2.071 \mathrm{E}+00$ & $7.197 \mathrm{E}+02$ & $1.609 \mathrm{E}+00$ & $7.202 \mathrm{E}+02$ \\
\hline
\end{tabular}

\begin{tabular}{|c|c|c|c|c|c|c|c|c|c|c|c|c|c|c|c|c|}
\hline $\begin{array}{c}\text { Group } \\
\text { Size } \\
\end{array}$ & $\alpha_{9}$ & $b_{9}$ & $\alpha_{10}$ & $b_{10}$ & $\alpha_{11}$ & $b_{11}$ & $\alpha_{12}$ & $b_{12}$ & $\alpha_{13}$ & $b_{13}$ & $\alpha_{14}$ & $b_{14}$ & $\alpha_{15}$ & $b_{15}$ & $\alpha_{16}$ & $b_{16}$ \\
\hline 9 & $8.859 \mathrm{E}-03$ & $2.043 \mathrm{E}+02$ & & & & & & & & & & & & & & \\
\hline 10 & $1.010 \mathrm{E}-01$ & $2.527 \mathrm{E}+02$ & $6.305 \mathrm{E}-03$ & $2.528 \mathrm{E}+02$ & & & & & & & & & & & & \\
\hline 11 & $2.985 \mathrm{E}-01$ & $3.078 \mathrm{E}+02$ & $7.511 \mathrm{E}-02$ & $3.080 \mathrm{E}+02$ & 4.489E-03 & $3.081 \mathrm{E}+02$ & & & & & & & & & & \\
\hline 12 & $4.567 \mathrm{E}-01$ & $3.174 \mathrm{E}+02$ & $2.045 \mathrm{E}-01$ & $3.176 \mathrm{E}+02$ & 4.782E-02 & $3.178 \mathrm{E}+02$ & 1.494E-02 & $3.178 \mathrm{E}+02$ & & & & & & & & \\
\hline 13 & $7.460 \mathrm{E}-01$ & $4.431 \mathrm{E}+02$ & $4.601 \mathrm{E}-01$ & $4.434 \mathrm{E}+02$ & $1.906 \mathrm{E}-01$ & $4.436 \mathrm{E}+02$ & 4.157E-02 & $4.438 \mathrm{E}+02$ & 2.293E-03 & $4.438 \mathrm{E}+02$ & & & & & & \\
\hline 14 & 9.219E-01 & $5.253 \mathrm{E}+02$ & 6.799E-01 & $5.255 \mathrm{E}+02$ & 3.934E-01 & $5.258 \mathrm{E}+02$ & $1.514 \mathrm{E}-01$ & $5.260 \mathrm{E}+02$ & 3.090E-02 & $5.261 \mathrm{E}+02$ & $1.643 \mathrm{E}-03$ & $5.262 \mathrm{E}+02$ & & & & \\
\hline 15 & $1.080 \mathrm{E}+00$ & $6.175 \mathrm{E}+02$ & $8.652 \mathrm{E}-01$ & $6.177 \mathrm{E}+02$ & $6.152 \mathrm{E}-01$ & $6.180 \mathrm{E}+02$ & 3.331E-01 & $6.183 E+02$ & $1.195 \mathrm{E}-01$ & $6.185 \mathrm{E}+02$ & 2.292E-02 & $6.186 \mathrm{E}+02$ & $1.177 \mathrm{E}-03$ & $6.186 \mathrm{E}+02$ & & \\
\hline 16 & $1.245 \mathrm{E}+00$ & $7.206 \mathrm{E}+02$ & $1.018 \mathrm{E}+00$ & 7.208E+02 & $8.140 \mathrm{E}-01$ & $7.210 \mathrm{E}+02$ & 5.510E-01 & $7.213 \mathrm{E}+02$ & 2.793E-01 & $7.215 \mathrm{E}+02$ & 9.387E-02 & $7.217 \mathrm{E}+02$ & $1.696 \mathrm{E}-02$ & $7.218 \mathrm{E}+02$ & 8.431E-04 & $7.218 \mathrm{E}+02$ \\
\hline
\end{tabular}




\subsection{Prior Distributions for the "Human" Cause Group (GH)}

To estimate prior distributions for the GH (i.e., Human) CCF cause group, the following selection criteria are defined in the CCF Database website:

- Type of CCF Event Level: All Level CCF Events

- CCF Event Type: CCF Events Only

- Date Range: 1997-2015

- Filter Independent Events by Selected Cause(s): True

- Shock Criteria: All Events

- Redundancy Range: Minimum = 2, Maximum $=16$

- Bayesian Update Method: Mean Method

- Failure Modes: select all failure modes except Setpoint

- CCF Categories: Cause $\rightarrow$ Human

A total of $67 \mathrm{CCF}$ events and 2,120.9 effective independent failure events related to the above selection criteria.

Additional criterion on CCF Categories $\rightarrow$ Degree $\rightarrow$ Almost/Partial or Complete is used to obtain the partial CCF events and complete CCF events, as required in the existing process. The unmapped/mapped impact vectors are also acquired from the CCF Database website. The mapped impact vectors for partial CCF events for each group size obtained from the website are used directly in the study.

Table 30 shows the number of partial CCF events, the number of complete CCF events, and the total number of $\mathrm{CCF}$ events for the GH cause group. The same binomial regression treatment used for complete $\mathrm{CCF}$ events was conducted. The estimated number of complete CCF events for each group size is listed in Table 30 .

Table 31 shows the mapped impact vectors for partial CCF events for the GH cause groups sized 216 obtained from the CCF Database website. Table 32 shows the adjusted $n_{k}$ results for CCF events in the GH cause groups sized 2-16, after adding the estimated number of complete CCF events. The MLEs or mean values of alpha factors for the GC cause groups of each size are then calculated, while the CalcPrior code is used to estimate the prior distributions for causal alpha factors pertaining to the $\mathrm{GH}$ cause group. Table 33 and Table 34 show the mean values and the distributions results, respectively.

Table 30. CCF data for the GH cause group from 1997 through 2015.

\begin{tabular}{|c|c|c|c|c|c|c|}
\hline $\begin{array}{c}\text { Group } \\
\text { Size }\end{array}$ & $\begin{array}{c}\text { No. } \\
\text { Partial } \\
\text { CCF } \\
\text { Events }\end{array}$ & $\begin{array}{c}\text { No. } \\
\text { Complete } \\
\text { CCF } \\
\text { Events }\end{array}$ & $\begin{array}{c}\text { Total No. } \\
\text { CCF } \\
\text { Events }\end{array}$ & $\begin{array}{c}\text { Prob. of } \\
\text { Complete } \\
\text { CCF } \\
\text { Event - } \\
\text { Data }\end{array}$ & $\begin{array}{c}\text { Prob. of } \\
\text { Complete } \\
\text { CCF Event - } \\
\text { Curve } \\
\text { Fitting }\end{array}$ & $\begin{array}{c}\text { Estimated } \\
\text { No. of } \\
\text { Complete } \\
\text { CCF Events }\end{array}$ \\
\hline 2 & 7 & 9 & 16 & 0.56250 & 0.46086 & 7.37377 \\
\hline 3 & 10 & 2 & 12 & 0.16667 & 0.25147 & 3.01769 \\
\hline 4 & 12 & 0 & 12 & 0.00000 & 0.12670 & 1.52042 \\
\hline 5 & 2 & 0 & 2 & 0.00000 & 0.06965 & 0.13930 \\
\hline 6 & 5 & 2 & 7 & 0.28571 & 0.04674 & 0.32719 \\
\hline 7 & 1 & 0 & 1 & 0.00000 & 0.03803 & 0.03803 \\
\hline
\end{tabular}




\begin{tabular}{|c|c|c|c|c|c|c|}
\hline $\begin{array}{c}\text { Group } \\
\text { Size }\end{array}$ & $\begin{array}{c}\text { No. } \\
\text { Partial } \\
\text { CCF } \\
\text { Events }\end{array}$ & $\begin{array}{c}\text { No. } \\
\text { Complete } \\
\text { CCF } \\
\text { Events }\end{array}$ & $\begin{array}{c}\text { Total No. } \\
\text { CCF } \\
\text { Events }\end{array}$ & $\begin{array}{c}\text { Prob. of } \\
\text { Complete } \\
\text { CCF } \\
\text { Event - } \\
\text { Data }\end{array}$ & $\begin{array}{c}\text { Prob. of } \\
\text { Complete } \\
\text { CCF Event - } \\
\text { Curve } \\
\text { Fitting }\end{array}$ & $\begin{array}{c}\text { Estimated } \\
\text { No. of } \\
\text { Complete } \\
\text { CCF Events }\end{array}$ \\
\hline 8 & 7 & 1 & 8 & 0.12500 & 0.03478 & 0.27825 \\
\hline 9 & 0 & 0 & 0 & NA & 0.03358 & 0.00000 \\
\hline 10 & 0 & 0 & 0 & NA & 0.03314 & 0.00000 \\
\hline 11 & 1 & 0 & 1 & 0.00000 & 0.03298 & 0.03298 \\
\hline 12 & 1 & 0 & 1 & 0.00000 & 0.03292 & 0.03292 \\
\hline 13 & 0 & 0 & 0 & NA & 0.03289 & 0.00000 \\
\hline 14 & 0 & 0 & 0 & NA & 0.03289 & 0.00000 \\
\hline 15 & 0 & 0 & 0 & NA & 0.03288 & 0.00000 \\
\hline 16 & 7 & 0 & 7 & 0.00000 & 0.03288 & 0.23018 \\
\hline Total & 53 & 14 & 67 & & & 12.99072 \\
\hline
\end{tabular}

Table 31. nk values for the partial CCF events in the GH cause group from 1997 through 2015.

\begin{tabular}{|c|c|c|c|c|c|c|c|c|c|c|c|c|c|c|c|c|}
\hline $\begin{array}{c}\text { Group } \\
\text { Size }\end{array}$ & $\mathrm{n}_{1}$ & $\mathrm{n}_{2}$ & $\mathrm{n}_{3}$ & $\mathrm{n}_{4}$ & $\mathrm{n}_{5}$ & $\mathrm{n}_{6}$ & $\mathrm{n}_{7}$ & $\mathrm{n}_{8}$ & $\mathrm{n}_{9}$ & $\mathrm{n}_{10}$ & $\mathrm{n}_{11}$ & $\mathrm{n}_{12}$ & $\mathrm{n}_{13}$ & $\mathrm{n}_{14}$ & $\mathrm{n}_{15}$ & $\mathrm{n}_{16}$ \\
\hline 2 & 29.035 & 8.039 & & & & & & & & & & & & & & \\
\hline 3 & 28.595 & 15.457 & 2.553 & & & & & & & & & & & & & \\
\hline 4 & 28.377 & 18.039 & 5.919 & 1.364 & & & & & & & & & & & & \\
\hline 5 & 31.267 & 15.626 & 8.745 & 3.540 & 0.776 & & & & & & & & & & & \\
\hline 6 & 33.916 & 14.356 & 9.281 & 5.454 & 2.124 & 0.475 & & & & & & & & & & \\
\hline 7 & 36.243 & 14.012 & 8.785 & 6.634 & 3.494 & 1.302 & 0.314 & & & & & & & & & \\
\hline 8 & 38.114 & 14.417 & 8.145 & 6.736 & 4.931 & 2.115 & 0.869 & 0.219 & & & & & & & & \\
\hline 9 & 39.948 & 15.045 & 7.649 & 6.459 & 5.260 & 3.406 & 1.456 & 0.605 & 0.158 & & & & & & & \\
\hline 10 & 41.428 & 15.943 & 7.375 & 6.056 & 5.324 & 4.052 & 2.370 & 1.022 & 0.441 & 0.1185 & & & & & & \\
\hline 11 & 42.595 & 16.998 & 7.320 & 5.640 & 5.193 & 4.359 & 3.072 & 1.660 & 0.739 & 0.3347 & 0.0907 & & & & & \\
\hline 12 & 43.705 & 17.835 & 7.554 & 5.277 & 4.949 & 4.443 & 3.523 & 2.293 & 1.176 & 0.5552 & 0.2630 & 0.0705 & & & & \\
\hline 13 & 44.805 & 18.423 & 8.015 & 5.008 & 4.658 & 4.376 & 3.761 & 2.799 & 1.692 & 0.8513 & 0.4341 & 0.2121 & 0.0554 & & & \\
\hline 14 & 45.757 & 18.996 & 8.564 & 4.849 & 4.368 & 4.218 & 3.836 & 3.141 & 2.183 & 1.2414 & 0.6358 & 0.3522 & 0.1740 & 0.0438 & & \\
\hline 15 & 46.587 & 19.527 & 9.179 & 4.793 & 4.107 & 4.013 & 3.798 & 3.328 & 2.577 & 1.6727 & 0.9154 & 0.4938 & 0.2945 & 0.1443 & 0.0348 & \\
\hline 16 & 47.317 & 19.999 & 9.844 & 4.826 & 3.890 & 3.793 & 3.688 & 3.390 & 2.847 & 2.0741 & 1.2657 & 0.6864 & 0.3999 & 0.2516 & 0.1205 & 0.0277 \\
\hline
\end{tabular}

Table 32. Adjusted nk values for CCF events in the GH cause group from 1997 through 2015.

\begin{tabular}{|c|c|c|c|c|c|c|c|c|c|c|c|c|c|c|c|c|c|c|}
\hline $\begin{array}{c}\text { Group } \\
\text { Size }\end{array}$ & $\mathrm{n}_{\mathrm{t}}$ & $\mathrm{n}_{\mathrm{I}}$ & $\mathrm{n}_{1}$ & $\mathrm{n}_{2}$ & $\mathrm{n}_{3}$ & $\mathrm{n}_{4}$ & $\mathrm{n}_{5}$ & $\mathrm{n}_{6}$ & $\mathrm{n}_{7}$ & $\mathrm{n}_{8}$ & $\mathrm{n}_{9}$ & $\mathrm{n}_{10}$ & $\mathrm{n}_{11}$ & $\mathrm{n}_{12}$ & $\mathrm{n}_{13}$ & $\mathrm{n}_{14}$ & $\mathrm{n}_{15}$ & $\mathrm{n}_{16}$ \\
\hline 2 & 804.338 & 759.890 & 29.035 & 15.413 & & & & & & & & & & & & & & \\
\hline 3 & 1189.463 & 1139.840 & 28.595 & 15.457 & 5.571 & & & & & & & & & & & & & \\
\hline 4 & 1575.010 & 1519.790 & 28.377 & 18.039 & 5.919 & 2.885 & & & & & & & & & & & & \\
\hline 5 & 1959.833 & 1899.740 & 31.267 & 15.626 & 8.745 & 3.540 & 0.915 & & & & & & & & & & & \\
\hline 6 & 2345.613 & 2279.680 & 33.916 & 14.356 & 9.281 & 5.454 & 2.124 & 0.802 & & & & & & & & & & \\
\hline
\end{tabular}




\begin{tabular}{|c|c|c|c|c|c|c|c|c|c|c|c|c|c|c|c|c|c|c|}
\hline $\begin{array}{c}\text { Group } \\
\text { Size }\end{array}$ & $\mathrm{n}_{\mathrm{t}}$ & $\mathrm{n}_{\mathrm{I}}$ & $\mathrm{n}_{1}$ & $\mathrm{n}_{2}$ & $\mathrm{n}_{3}$ & $\mathrm{n}_{4}$ & $\mathrm{n}_{5}$ & $\mathrm{n}_{6}$ & $\mathrm{n}_{7}$ & $\mathrm{n}_{8}$ & $\mathrm{n}_{9}$ & $\mathrm{n}_{10}$ & $\mathrm{n}_{11}$ & $\mathrm{n}_{12}$ & $\mathrm{n}_{13}$ & $\mathrm{n}_{14}$ & $\mathrm{n}_{15}$ & $\mathrm{n}_{16}$ \\
\hline 7 & 2730.451 & 2659.630 & 36.243 & 14.012 & 8.785 & 6.634 & 3.494 & 1.302 & 0.352 & & & & & & & & & \\
\hline 8 & 3115.405 & 3039.580 & 38.114 & 14.417 & 8.145 & 6.736 & 4.931 & 2.115 & 0.869 & 0.497 & & & & & & & & \\
\hline 9 & 3499.516 & 3419.530 & 39.948 & 15.045 & 7.649 & 6.459 & 5.260 & 3.406 & 1.456 & 0.605 & 0.158 & & & & & & & \\
\hline 10 & 3883.599 & 3799.470 & 41.428 & 15.943 & 7.375 & 6.056 & 5.324 & 4.052 & 2.370 & 1.022 & 0.441 & 0.119 & & & & & & \\
\hline 11 & 4267.455 & 4179.420 & 42.595 & 16.998 & 7.320 & 5.640 & 5.193 & 4.359 & 3.072 & 1.660 & 0.739 & 0.335 & 0.124 & & & & & \\
\hline 12 & 4651.047 & 4559.370 & 43.705 & 17.835 & 7.554 & 5.277 & 4.949 & 4.443 & 3.523 & 2.293 & 1.176 & 0.555 & 0.263 & 0.103 & & & & \\
\hline 13 & 5034.399 & 4939.310 & 44.805 & 18.423 & 8.015 & 5.008 & 4.658 & 4.376 & 3.761 & 2.799 & 1.692 & 0.851 & 0.434 & 0.212 & 0.055 & & & \\
\hline 14 & 5417.618 & 5319.260 & 45.757 & 18.996 & 8.564 & 4.849 & 4.368 & 4.218 & 3.836 & 3.141 & 2.183 & 1.241 & 0.636 & 0.352 & 0.174 & 0.044 & & \\
\hline 15 & 5800.674 & 5699.210 & 46.587 & 19.527 & 9.179 & 4.793 & 4.107 & 4.013 & 3.798 & 3.328 & 2.577 & 1.673 & 0.915 & 0.494 & 0.294 & 0.144 & 0.035 & \\
\hline 16 & 6183.811 & 6079.160 & 47.317 & 19.999 & 9.844 & 4.826 & 3.890 & 3.793 & 3.688 & 3.390 & 2.847 & 2.074 & 1.266 & 0.686 & 0.400 & 0.252 & 0.120 & 0.258 \\
\hline
\end{tabular}

Table 33. Calculated alpha factor mean values for CCF events in the GH cause group from 1997 through 2015.

\begin{tabular}{|c|c|c|c|c|c|c|c|c|c|c|c|c|c|c|c|c|}
\hline \begin{tabular}{|c|} 
Group \\
Size \\
\end{tabular} & $\alpha_{1}$ & $\alpha_{2}$ & $\alpha_{3}$ & $\alpha_{4}$ & $\alpha_{5}$ & $\alpha_{6}$ & $\alpha_{7}$ & $\alpha_{8}$ & $\alpha_{9}$ & $\alpha_{10}$ & $\alpha_{11}$ & $\alpha_{12}$ & $\alpha_{13}$ & $\alpha_{14}$ & $\alpha_{15}$ & $\alpha_{16}$ \\
\hline 2 & 0.9808 & $1.916 \mathrm{E}-02$ & & & & & & & & & & & & & & \\
\hline 3 & 0.9823 & $1.299 \mathrm{E}-02$ & $4.684 \mathrm{E}-03$ & & & & & & & & & & & & & \\
\hline 4 & 0.9830 & $1.145 \mathrm{E}-02$ & $3.758 \mathrm{E}-03$ & $1.832 \mathrm{E}-03$ & & & & & & & & & & & & \\
\hline 5 & 0.9853 & 7.973E-03 & $4.462 \mathrm{E}-03$ & $1.806 \mathrm{E}-03$ & 4.669E-04 & & & & & & & & & & & \\
\hline 6 & 0.9864 & $6.120 \mathrm{E}-03$ & $3.957 \mathrm{E}-03$ & $2.325 \mathrm{E}-03$ & 9.056E-04 & $3.419 \mathrm{E}-04$ & & & & & & & & & & \\
\hline 7 & 0.9873 & 5.132E-03 & $3.217 \mathrm{E}-03$ & $2.430 \mathrm{E}-03$ & $1.279 \mathrm{E}-03$ & $4.767 \mathrm{E}-04$ & $1.290 \mathrm{E}-04$ & & & & & & & & & \\
\hline 8 & 0.9879 & $4.628 \mathrm{E}-03$ & $2.615 \mathrm{E}-03$ & $2.162 \mathrm{E}-03$ & $1.583 \mathrm{E}-03$ & $6.790 \mathrm{E}-04$ & $2.789 \mathrm{E}-04$ & $1.595 \mathrm{E}-04$ & & & & & & & & \\
\hline 9 & 0.9886 & 4.299E- 03 & $2.186 \mathrm{E}-03$ & $1.846 \mathrm{E}-03$ & $1.503 \mathrm{E}-03$ & $9.732 \mathrm{E}-04$ & $4.159 \mathrm{E}-04$ & $1.729 \mathrm{E}-04$ & $4.527 \mathrm{E}-05$ & & & & & & & \\
\hline 10 & 0.9890 & $4.105 \mathrm{E}-03$ & $1.899 \mathrm{E}-03$ & $1.559 \mathrm{E}-03$ & $1.371 \mathrm{E}-03$ & $1.043 \mathrm{E}-03$ & $6.103 \mathrm{E}-04$ & $2.632 \mathrm{E}-04$ & $1.135 \mathrm{E}-04$ & 3.052E-05 & & & & & & \\
\hline 11 & 0.9894 & 3.983E-03 & $1.715 \mathrm{E}-03$ & $1.322 \mathrm{E}-03$ & $1.217 \mathrm{E}-03$ & $1.022 \mathrm{E}-03$ & 7.199E-04 & $3.890 \mathrm{E}-04$ & $1.732 \mathrm{E}-04$ & 7.842E-05 & $2.899 \mathrm{E}-05$ & & & & & \\
\hline 12 & 0.9897 & 3.835E-03 & $1.624 \mathrm{E}-03$ & $1.135 \mathrm{E}-03$ & $1.064 \mathrm{E}-03$ & $\mid 9.552 \mathrm{E}-04$ & 7.575E-04 & $4.931 \mathrm{E}-04$ & $2.529 \mathrm{E}-04$ & 1.194E-04 & $5.655 \mathrm{E}-05$ & $2.224 \mathrm{E}-05$ & & & & \\
\hline 13 & 0.9900 & 3.659E-03 & $1.592 \mathrm{E}-03$ & 9.948E-04 & 9.253E-04 & $8.691 \mathrm{E}-04$ & 7.471E-04 & $5.560 \mathrm{E}-04$ & $3.360 \mathrm{E}-04$ & $1.691 \mathrm{E}-04$ & 8.622E- 05 & $4.212 \mathrm{E}-05$ & 1.100E-05 & & & \\
\hline 14 & 0.9903 & $3.506 \mathrm{E}-03$ & $1.581 \mathrm{E}-03$ & $8.951 \mathrm{E}-04$ & $8.062 \mathrm{E}-04$ & 7.785E-04 & 7.081E-04 & $5.797 \mathrm{E}-04$ & $4.029 \mathrm{E}-04$ & 2.291E-04 & $1.174 \mathrm{E}-04$ & $6.501 \mathrm{E}-05$ & $3.212 \mathrm{E}-05$ & $8.085 \mathrm{E}-06$ & & \\
\hline 15 & 0.9905 & $3.366 \mathrm{E}-03$ & $1.582 \mathrm{E}-03$ & $8.262 \mathrm{E}-04$ & 7.079E-04 & 6.919E-04 & $6.548 \mathrm{E}-04$ & $5.737 \mathrm{E}-04$ & $4.443 \mathrm{E}-04$ & 2.884E-04 & $1.578 \mathrm{E}-04$ & $8.513 \mathrm{E}-05$ & $5.077 \mathrm{E}-05$ & $2.488 \mathrm{E}-05$ & $5.997 \mathrm{E}-06$ & \\
\hline 16 & 0.9907 & $3.234 \mathrm{E}-03$ & $1.592 \mathrm{E}-03$ & 7.804E-04 & 6.291E-04 & 6.134E-04 & $5.965 \mathrm{E}-04$ & $5.483 \mathrm{E}-04$ & $4.604 \mathrm{E}-04$ & 3.354E-04 & $2.047 \mathrm{E}-04$ & $1.110 \mathrm{E}-04$ & $6.467 \mathrm{E}-05$ & $4.069 \mathrm{E}-05$ & $1.948 \mathrm{E}-05$ & $4.170 \mathrm{E}-05$ \\
\hline
\end{tabular}


Table 34. Estimated CCF industry-wide prior distributions with CCF events in the GH cause group from 1997 through 2015.

\begin{tabular}{|c|c|c|c|c|c|c|c|c|c|c|c|c|c|c|c|c|}
\hline $\begin{array}{c}\text { Group } \\
\text { Size }\end{array}$ & $\alpha_{1}$ & $\mathrm{~b}_{1}$ & $\alpha_{2}$ & $\mathrm{~b}_{2}$ & $\alpha_{3}$ & $b_{3}$ & $\alpha_{4}$ & $\mathrm{~b}_{4}$ & $\alpha_{5}$ & $b_{5}$ & $\alpha_{6}$ & $\mathrm{~b}_{6}$ & $\alpha_{7}$ & $\mathrm{~b}_{7}$ & $\alpha_{8}$ & $\mathrm{~b}_{8}$ \\
\hline 2 & $2.409 \mathrm{E}+01$ & 4.706E-01 & $4.706 \mathrm{E}-01$ & $2.409 \mathrm{E}+01$ & & & & & & & & & & & & \\
\hline 3 & $6.126 \mathrm{E}+01$ & $1.103 \mathrm{E}+00$ & $8.104 \mathrm{E}-01$ & $6.155 \mathrm{E}+01$ & $2.921 \mathrm{E}-01$ & $6.207 \mathrm{E}+01$ & & & & & & & & & & \\
\hline 4 & $1.126 \mathrm{E}+02$ & $1.953 \mathrm{E}+00$ & $1.312 \mathrm{E}+00$ & $1.133 \mathrm{E}+02$ & $4.307 \mathrm{E}-01$ & $1.142 \mathrm{E}+02$ & $2.099 \mathrm{E}-01$ & $1.144 \mathrm{E}+02$ & & & & & & & & \\
\hline 5 & $2.082 \mathrm{E}+02$ & $3.107 \mathrm{E}+00$ & $1.684 \mathrm{E}+00$ & $2.096 \mathrm{E}+02$ & 9.426E-01 & $2.103 \mathrm{E}+02$ & $3.816 \mathrm{E}-01$ & $2.109 \mathrm{E}+02$ & $9.865 \mathrm{E}-02$ & $2.112 \mathrm{E}+02$ & & & & & & \\
\hline 6 & $2.761 \mathrm{E}+02$ & $3.821 \mathrm{E}+00$ & $1.714 \mathrm{E}+00$ & $2.783 \mathrm{E}+02$ & $1.108 \mathrm{E}+00$ & $2.789 \mathrm{E}+02$ & $6.509 \mathrm{E}-01$ & $2.793 \mathrm{E}+02$ & $2.536 \mathrm{E}-01$ & $2.797 \mathrm{E}+02$ & 9.572E-02 & $2.799 \mathrm{E}+02$ & & & & \\
\hline 7 & $4.050 \mathrm{E}+02$ & $5.195 \mathrm{E}+00$ & $2.105 \mathrm{E}+00$ & $4.081 \mathrm{E}+02$ & $1.320 \mathrm{E}+00$ & $4.089 \mathrm{E}+02$ & $9.967 \mathrm{E}-01$ & $4.092 \mathrm{E}+02$ & $5.249 \mathrm{E}-01$ & $4.097 \mathrm{E}+02$ & $1.956 \mathrm{E}-01$ & $4.100 \mathrm{E}+02$ & 5.291E-02 & $4.102 \mathrm{E}+02$ & & \\
\hline 8 & $4.759 \mathrm{E}+02$ & $|5.832 \mathrm{E}+00|$ & $2.229 \mathrm{E}+00$ & $4.795 \mathrm{E}+02$ & $1.260 \mathrm{E}+00$ & $4.805 \mathrm{E}+02$ & $1.042 \mathrm{E}+00$ & $4.807 \mathrm{E}+02$ & 7.626E-01 & $4.810 \mathrm{E}+02$ & 3.271E-01 & $4.814 \mathrm{E}+02$ & $1.344 \mathrm{E}-01$ & $4.816 \mathrm{E}+02$ & 7.682E-02 & $4.817 \mathrm{E}+02$ \\
\hline 9 & $6.722 \mathrm{E}+02$ & $7.779 \mathrm{E}+00$ & $2.923 \mathrm{E}+00$ & $6.770 \mathrm{E}+02$ & $1.486 \mathrm{E}+00$ & $6.785 \mathrm{E}+02$ & $1.255 \mathrm{E}+00$ & $6.787 \mathrm{E}+02$ & $1.022 \mathrm{E}+00 \mid$ & $6.789 \mathrm{E}+02$ & 6.617E-01 & $6.793 \mathrm{E}+02$ & $2.828 \mathrm{E}-01$ & $6.797 \mathrm{E}+02$ & $1.176 \mathrm{E}-01$ & $6.798 \mathrm{E}+0$ \\
\hline 10 & $8.249 \mathrm{E}+02$ & $9.171 \mathrm{E}+00 \mid$ & $3.424 \mathrm{E}+00$ & $8.307 \mathrm{E}+02$ & $1.584 \mathrm{E}+00$ & $8.325 \mathrm{E}+02$ & $1.301 \mathrm{E}+00$ & $8.328 \mathrm{E}+02$ & $1.143 \mathrm{E}+00$ & $8.330 \mathrm{E}+02$ & 8.702E-01 & $8.332 \mathrm{E}+02$ & 5.090E-01 & $8.336 \mathrm{E}+02$ & 2.195E-01 & $8.339 \mathrm{E}+02$ \\
\hline 11 & $9.631 \mathrm{E}+02$ & $1.037 \mathrm{E}+01$ & $3.878 \mathrm{E}+00$ & $9.696 \mathrm{E}+02$ & $1.670 \mathrm{E}+00$ & $9.718 \mathrm{E}+02$ & $1.287 \mathrm{E}+00$ & $9.722 \mathrm{E}+02$ & $1.185 \mathrm{E}+00 \mid$ & $9.723 \mathrm{E}+02$ & 9.944E-01 & $9.725 \mathrm{E}+02$ & 7.008E-01 & $9.728 \mathrm{E}+02$ & 3.786E-01 & $9.731 \mathrm{E}+02$ \\
\hline 12 & $1.138 \mathrm{E}+03$ & $1.186 \mathrm{E}+01$ & $4.409 \mathrm{E}+00$ & $1.146 \mathrm{E}+03$ & $1.868 \mathrm{E}+00$ & $1.148 \mathrm{E}+03$ & $1.305 \mathrm{E}+00$ & $1.149 \mathrm{E}+03$ & $1.224 \mathrm{E}+00$ & $1.149 \mathrm{E}+03$ & $1.098 \mathrm{E}+00$ & $1.149 \mathrm{E}+03$ & 8.711E-01 & $1.149 \mathrm{E}+03$ & 5.670E-01 & $1.149 \mathrm{E}+0$ \\
\hline 13 & $1.380 \mathrm{E}+03$ & $1.393 \mathrm{E}+01$ & $5.102 \mathrm{E}+00$ & $1.389 \mathrm{E}+03$ & $2.219 \mathrm{E}+00$ & $1.392 \mathrm{E}+03$ & $1.387 \mathrm{E}+00$ & $1.393 \mathrm{E}+03$ & $1.290 \mathrm{E}+00$ & $1.393 \mathrm{E}+03$ & $1.212 \mathrm{E}+00$ & $1.393 \mathrm{E}+03$ & $1.042 \mathrm{E}+00$ & $1.393 \mathrm{E}+03$ & 7.751E-01 & $1.393 \mathrm{E}+03$ \\
\hline 14 & $1.599 \mathrm{E}+03$ & $1.568 \mathrm{E}+01$ & $5.662 \mathrm{E}+00$ & $1.609 \mathrm{E}+03$ & $2.552 \mathrm{E}+00$ & $1.612 \mathrm{E}+03$ & $1.445 \mathrm{E}+00$ & $1.613 \mathrm{E}+03$ & $1.302 \mathrm{E}+00$ & $1.613 \mathrm{E}+03$ & $1.257 \mathrm{E}+00$ & $1.613 \mathrm{E}+03$ & $1.143 \mathrm{E}+00$ & $1.614 \mathrm{E}+03$ & 9.361E-01 & $1.614 \mathrm{E}+03$ \\
\hline 15 & $1.835 \mathrm{E}+03$ & $1.753 \mathrm{E}+01$ & $6.237 \mathrm{E}+00$ & $1.847 \mathrm{E}+03$ & $2.932 \mathrm{E}+00$ & $1.850 \mathrm{E}+03$ & $1.531 \mathrm{E}+00$ & $1.851 \mathrm{E}+03$ & $1.312 \mathrm{E}+00$ & $1.852 \mathrm{E}+03$ & $1.282 \mathrm{E}+00$ & $1.852 \mathrm{E}+03$ & $1.213 \mathrm{E}+00$ & $1.852 \mathrm{E}+03$ & $1.063 \mathrm{E}+00$ & $1.852 \mathrm{E}+03$ \\
\hline 16 & $1.801 \mathrm{E}+03$ & $1.685 \mathrm{E}+01$ & $5.878 \mathrm{E}+00$ & $1.812 \mathrm{E}+03$ & $2.893 \mathrm{E}+00$ & $1.815 \mathrm{E}+03$ & $1.418 \mathrm{E}+00$ & $1.816 \mathrm{E}+03$ & $1.144 \mathrm{E}+00$ & $1.816 \mathrm{E}+03$ & $1.115 \mathrm{E}+00$ & $1.816 \mathrm{E}+03$ & $1.084 \mathrm{E}+00$ & $1.817 \mathrm{E}+03$ & 9.965E-01 & $1.817 \mathrm{E}+\mathrm{C}$ \\
\hline
\end{tabular}

\begin{tabular}{|c|c|c|c|c|c|c|c|c|c|c|c|c|c|c|c|c|}
\hline $\begin{array}{c}\text { Group } \\
\text { Size }\end{array}$ & $\alpha_{9}$ & $\mathrm{~b}_{9}$ & $\alpha_{10}$ & $\mathrm{~b}_{10}$ & $\alpha_{11}$ & $b_{11}$ & $\alpha_{12}$ & $b_{12}$ & $\alpha_{13}$ & $b_{13}$ & $\alpha_{14}$ & $\mathrm{~b}_{14}$ & $\alpha_{15}$ & $b_{15}$ & $\alpha_{16}$ & $b_{16}$ \\
\hline 9 & 3.078E-02 & $6.799 \mathrm{E}+02$ & & & & & & & & & & & & & & \\
\hline 10 & 9.465E-02 & $8.340 \mathrm{E}+02 \mid$ & $2.546 \mathrm{E}-02$ & $8.341 \mathrm{E}+02$ & & & & & & & & & & & & \\
\hline 11 & $1.686 \mathrm{E}-01$ & $9.733 \mathrm{E}+02$ & 7.634E-02 & $9.734 \mathrm{E}+02$ & 2.822E-02 & $9.735 \mathrm{E}+02$ & & & & & & & & & & \\
\hline 12 & $2.908 \mathrm{E}-01$ & $1.150 \mathrm{E}+03$ & $1.373 \mathrm{E}-01$ & $1.150 \mathrm{E}+03$ & $6.503 \mathrm{E}-02$ & $1.150 \mathrm{E}+03$ & $2.558 \mathrm{E}-02$ & $1.150 \mathrm{E}+03$ & & & & & & & & \\
\hline 13 & $4.684 \mathrm{E}-01$ & $1.394 \mathrm{E}+03$ & $2.357 \mathrm{E}-01$ & $1.394 \mathrm{E}+03$ & $1.202 \mathrm{E}-01$ & $1.394 \mathrm{E}+03$ & $5.873 \mathrm{E}-02$ & $1.394 \mathrm{E}+03$ & $1.534 \mathrm{E}-02$ & $1.394 \mathrm{E}+03$ & & & & & & \\
\hline 14 & $6.505 \mathrm{E}-01$ & $1.614 \mathrm{E}+03$ & $3.700 \mathrm{E}-01$ & $1.614 \mathrm{E}+03$ & $1.895 \mathrm{E}-01$ & $1.615 \mathrm{E}+03$ & $1.050 \mathrm{E}-01$ & $1.615 \mathrm{E}+03$ & 5.186E-02 & $1.615 \mathrm{E}+03$ & $1.306 \mathrm{E}-02$ & $1.615 \mathrm{E}+03$ & & & & \\
\hline 15 & $8.233 \mathrm{E}-01$ & $1.852 \mathrm{E}+03$ & 5.343E-01 & $1.852 \mathrm{E}+03$ & $2.924 \mathrm{E}-01$ & $1.853 \mathrm{E}+03$ & $1.577 \mathrm{E}-01$ & $1.853 \mathrm{E}+03$ & $9.406 \mathrm{E}-02$ & $1.853 \mathrm{E}+03$ & $4.610 \mathrm{E}-02$ & $1.853 \mathrm{E}+03$ & $1.111 \mathrm{E}-02$ & $1.853 \mathrm{E}+03$ & & \\
\hline 16 & $8.368 \mathrm{E}-01$ & $1.817 \mathrm{E}+03$ & $6.096 \mathrm{E}-01$ & $1.817 \mathrm{E}+03$ & 3.720E-01 & $1.817 \mathrm{E}+03$ & 2.017E-01 & $1.817 \mathrm{E}+03$ & $1.175 \mathrm{E}-01$ & $1.817 \mathrm{E}+03$ & 7.395E-02 & $1.818 \mathrm{E}+03$ & $3.541 \mathrm{E}-02$ & $1.818 \mathrm{E}+03$ & 7.580E-02 & $1.818 \mathrm{E}+03$ \\
\hline
\end{tabular}




\subsection{Prior Distributions for the "Other" Cause Group (GO)}

To estimate prior distributions for the GO (i.e., Other or unknown) CCF cause group, the following selection criteria are defined in the CCF Database website:

- Type of CCF Event Level: All Level CCF Events

- CCF Event Type: CCF Events Only

- Date Range: 1997-2015

- Filter Independent Events by Selected Cause(s): True

- Shock Criteria: All Events

- Redundancy Range: Minimum = 2, Maximum $=16$

- Bayesian Update Method: Mean Method

- Failure Modes: select all failure modes except Setpoint

- CCF Categories: Cause $\rightarrow$ Other \& Unknown

A total of nine CCF events and 921.8 effective independent failure events related to the above selection criteria.

Additional criterion on CCF Categories $\rightarrow$ Degree $\rightarrow$ Almost/Partial or Complete is used to obtain the partial CCF events and complete CCF events, as required in the existing process. The unmapped/mapped impact vectors are also acquired from the CCF Database website. The mapped impact vectors for partial CCF events for each group size obtained from the website are used directly in the study.

Table 35 shows the number of partial CCF events, the number of complete CCF events, and the total number of $\mathrm{CCF}$ events for the GO cause group. The same binomial regression treatment used for complete $\mathrm{CCF}$ events was conducted. The estimated number of complete CCF events for each group size is listed in Table 35.

Table 36 shows the mapped impact vectors for partial CCF events for the GO cause groups sized 216 obtained from the CCF Database website. Table 37 shows the adjusted $n_{k}$ results for CCF events in the GO cause groups sized 2-16, after adding the estimated number of complete CCF events. The MLEs or mean values of alpha factors for the GC cause groups of each size are then calculated, while the CalcPrior code is used to estimate the prior distributions for causal alpha factors pertaining to the GO cause group. Table 38 and Table 39 show the mean values and the distributions results, respectively.

Table 35. CCF data for the GO cause group from 1997 through 2015.

\begin{tabular}{|c|c|c|c|c|c|c|}
\hline $\begin{array}{c}\text { Group } \\
\text { Size }\end{array}$ & $\begin{array}{c}\text { No. } \\
\text { Partial } \\
\text { CCF } \\
\text { Events }\end{array}$ & $\begin{array}{c}\text { No. } \\
\text { Complete } \\
\text { CCF } \\
\text { Events }\end{array}$ & $\begin{array}{c}\text { Total No. } \\
\text { CCF } \\
\text { Events }\end{array}$ & $\begin{array}{c}\text { Prob. of } \\
\text { Complete } \\
\text { CCF } \\
\text { Event - } \\
\text { Data }\end{array}$ & $\begin{array}{c}\text { Prob. of } \\
\text { Complete } \\
\text { CCF Event - } \\
\text { Curve } \\
\text { Fitting }\end{array}$ & $\begin{array}{c}\text { Estimated } \\
\text { No. of } \\
\text { Complete } \\
\text { CCF Events }\end{array}$ \\
\hline 2 & 0 & 2 & 2 & 1.00000 & 0.58154 & 1.16309 \\
\hline 3 & 2 & 0 & 2 & 0.00000 & 0.37068 & 0.74135 \\
\hline 4 & 2 & 0 & 2 & 0.00000 & 0.22746 & 0.45492 \\
\hline 5 & 0 & 0 & 0 & NA & 0.15688 & 0.00000 \\
\hline 6 & 1 & 0 & 1 & 0.00000 & 0.12755 & 0.12755 \\
\hline 7 & 0 & 0 & 0 & NA & 0.11624 & 0.00000 \\
\hline
\end{tabular}




\begin{tabular}{|c|c|c|c|c|c|c|}
\hline $\begin{array}{c}\text { Group } \\
\text { Size }\end{array}$ & $\begin{array}{c}\text { No. } \\
\text { Partial } \\
\text { CCF } \\
\text { Events }\end{array}$ & $\begin{array}{c}\text { No. } \\
\text { Complete } \\
\text { CCF } \\
\text { Events }\end{array}$ & $\begin{array}{c}\text { Total No. } \\
\text { CCF } \\
\text { Events }\end{array}$ & $\begin{array}{c}\text { Prob. of } \\
\text { Complete } \\
\text { CCF } \\
\text { Event - } \\
\text { Data }\end{array}$ & $\begin{array}{c}\text { Prob. of } \\
\text { Complete } \\
\text { CCF Event - } \\
\text { Curve } \\
\text { Fitting }\end{array}$ & $\begin{array}{c}\text { Estimated } \\
\text { No. of } \\
\text { Complete } \\
\text { CCF Events }\end{array}$ \\
\hline 8 & 1 & 1 & 2 & 0.50000 & 0.11201 & 0.22402 \\
\hline 9 & 0 & 0 & 0 & NA & 0.11044 & 0.00000 \\
\hline 10 & 0 & 0 & 0 & NA & 0.10986 & 0.00000 \\
\hline 11 & 0 & 0 & 0 & NA & 0.10965 & 0.00000 \\
\hline 12 & 0 & 0 & 0 & NA & 0.10957 & 0.00000 \\
\hline 13 & 0 & 0 & 0 & NA & 0.10954 & 0.00000 \\
\hline 14 & 0 & 0 & 0 & NA & 0.10953 & 0.00000 \\
\hline 15 & 0 & 0 & 0 & NA & 0.10953 & 0.00000 \\
\hline 16 & 0 & 0 & 0 & NA & 0.10953 & 0.00000 \\
\hline Total & 6 & 3 & 9 & & & 2.71094 \\
\hline
\end{tabular}

Table 36. nk values for the partial CCF events in the GO cause group from 1997 through 2015.

\begin{tabular}{|c|c|c|c|c|c|c|c|c|c|c|c|c|c|c|c|c|}
\hline $\begin{array}{c}\text { Group } \\
\text { Size }\end{array}$ & $\mathrm{n}_{1}$ & $\mathrm{n}_{2}$ & $\mathrm{n}_{3}$ & $\mathrm{n}_{4}$ & $\mathrm{n}_{5}$ & $\mathrm{n}_{6}$ & $\mathrm{n}_{7}$ & $\mathrm{n}_{8}$ & $\mathrm{n}_{9}$ & $\mathrm{n}_{10}$ & $\mathrm{n}_{11}$ & $\mathrm{n}_{12}$ & $\mathrm{n}_{13}$ & $\mathrm{n}_{14}$ & $\mathrm{n}_{15}$ & $\mathrm{n}_{16}$ \\
\hline 2 & 3.491 & 0.738 & & & & & & & & & & & & & & \\
\hline 3 & 3.428 & 1.809 & 0.135 & & & & & & & & & & & & & \\
\hline 4 & 2.905 & 2.672 & 0.456 & 0.064 & & & & & & & & & & & & \\
\hline 5 & 2.672 & 2.701 & 1.007 & 0.220 & 0.032 & & & & & & & & & & & \\
\hline 6 & 2.264 & 2.878 & 1.241 & 0.497 & 0.114 & 0.016 & & & & & & & & & & \\
\hline 7 & 2.161 & 2.591 & 1.496 & 0.739 & 0.262 & 0.061 & 0.008 & & & & & & & & & \\
\hline 8 & 2.032 & 2.403 & 1.580 & 0.940 & 0.433 & 0.146 & 0.033 & 0.004 & & & & & & & & \\
\hline 9 & 1.960 & 2.188 & 1.607 & 1.076 & 0.597 & 0.258 & 0.084 & 0.018 & 0.002 & & & & & & & \\
\hline 10 & 1.887 & 2.020 & 1.585 & 1.157 & 0.732 & 0.381 & 0.157 & 0.049 & 0.010 & 0.0010 & & & & & & \\
\hline 11 & 1.815 & 1.888 & 1.541 & 1.195 & 0.832 & 0.497 & 0.245 & 0.097 & 0.029 & 0.0054 & 0.0005 & & & & & \\
\hline 12 & 1.744 & 1.781 & 1.488 & 1.204 & 0.899 & 0.596 & 0.337 & 0.159 & 0.060 & 0.0168 & 0.0030 & 0.0002 & & & & \\
\hline 13 & 1.674 & 1.693 & 1.435 & 1.195 & 0.940 & 0.673 & 0.425 & 0.230 & 0.104 & 0.0375 & 0.0098 & 0.0016 & 0.0001 & & & \\
\hline 14 & 1.605 & 1.619 & 1.385 & 1.176 & 0.959 & 0.730 & 0.501 & 0.302 & 0.158 & 0.0686 & 0.0232 & 0.0056 & 0.0009 & 0.0001 & & \\
\hline 15 & 1.539 & 1.555 & 1.338 & 1.151 & 0.965 & 0.767 & 0.563 & 0.370 & 0.216 & 0.1085 & 0.0448 & 0.0143 & 0.0032 & 0.0005 & 0.0000 & \\
\hline 16 & 1.474 & 1.499 & 1.296 & 1.124 & 0.961 & 0.790 & 0.610 & 0.431 & 0.274 & 0.1543 & 0.0744 & 0.0291 & 0.0087 & 0.0018 & 0.0002 & 0.0000 \\
\hline
\end{tabular}

Table 37. Adjusted nk values for CCF events in the GO cause group from 1997 through 2015.

\begin{tabular}{|c|c|c|c|c|c|c|c|c|c|c|c|c|c|c|c|c|c|c|}
\hline $\begin{array}{c}\text { Group } \\
\text { Size }\end{array}$ & $\mathrm{n}_{\mathrm{t}}$ & $\mathrm{n}_{\mathrm{I}}$ & $\mathrm{n}_{1}$ & $\mathrm{n}_{2}$ & $\mathrm{n}_{3}$ & $\mathrm{n}_{4}$ & $\mathrm{n}_{5}$ & $\mathrm{n}_{6}$ & $\mathrm{n}_{7}$ & $\mathrm{n}_{8}$ & $\mathrm{n}_{9}$ & $\mathrm{n}_{10}$ & $\mathrm{n}_{11}$ & $\mathrm{n}_{12}$ & $\mathrm{n}_{13}$ & $\mathrm{n}_{14}$ & $\mathrm{n}_{15}$ & $\mathrm{n}_{16}$ \\
\hline 2 & 420.20 & 414.81 & 3.491 & 1.901 & & & & & & & & & & & & & & \\
\hline 3 & 628.32 & 622.21 & 3.428 & 1.809 & 0.876 & & & & & & & & & & & & & \\
\hline 4 & 836.17 & 829.62 & 2.905 & 2.672 & 0.456 & 0.519 & & & & & & & & & & & & \\
\hline 5 & 1043.66 & 1037.03 & 2.672 & 2.701 & 1.007 & 0.220 & 0.032 & & & & & & & & & & & \\
\hline 6 & 1251.57 & 1244.43 & 2.264 & 2.878 & 1.241 & 0.497 & 0.114 & 0.143 & & & & & & & & & & \\
\hline
\end{tabular}




\begin{tabular}{|c|c|c|c|c|c|c|c|c|c|c|c|c|c|c|c|c|c|c|}
\hline $\begin{array}{c}\text { Group } \\
\text { Size }\end{array}$ & $\mathrm{n}_{\mathrm{t}}$ & $\mathrm{n}_{\mathrm{I}}$ & $\mathrm{n}_{1}$ & $\mathrm{n}_{2}$ & $\mathrm{n}_{3}$ & $\mathrm{n}_{4}$ & $\mathrm{n}_{5}$ & $\mathrm{n}_{6}$ & $\mathrm{n}_{7}$ & $\mathrm{n}_{8}$ & $\mathrm{n}_{9}$ & $\mathrm{n}_{10}$ & $\mathrm{n}_{11}$ & $\mathrm{n}_{12}$ & $\mathrm{n}_{13}$ & $\mathrm{n}_{14}$ & $\mathrm{n}_{15}$ & $\mathrm{n}_{16}$ \\
\hline 7 & 1459.16 & 1451.84 & 2.161 & 2.591 & 1.496 & 0.739 & 0.262 & 0.061 & 0.008 & & & & & & & & & \\
\hline 8 & 1667.04 & 1659.24 & 2.032 & 2.403 & 1.580 & 0.940 & 0.433 & 0.146 & 0.033 & 0.228 & & & & & & & & \\
\hline 9 & 1874.44 & 1866.65 & 1.960 & 2.188 & 1.607 & 1.076 & 0.597 & 0.258 & 0.084 & 0.018 & 0.002 & & & & & & & \\
\hline 10 & 2082.03 & 2074.05 & 1.887 & 2.020 & 1.585 & 1.157 & 0.732 & 0.381 & 0.157 & 0.049 & 0.010 & 0.001 & & & & & & \\
\hline 11 & 2289.60 & 2281.46 & 1.815 & 1.888 & 1.541 & 1.195 & 0.832 & 0.497 & 0.245 & 0.097 & 0.029 & 0.005 & 0.000 & & & & & \\
\hline 12 & 2497.15 & 2488.86 & 1.744 & 1.781 & 1.488 & 1.204 & 0.899 & 0.596 & 0.337 & 0.159 & 0.060 & 0.017 & 0.003 & 0.000 & & & & \\
\hline 13 & 2704.68 & 2696.26 & 1.674 & 1.693 & 1.435 & 1.195 & 0.940 & 0.673 & 0.425 & 0.230 & 0.104 & 0.038 & 0.010 & 0.002 & 0.000 & & & \\
\hline 14 & 2912.20 & 2903.67 & 1.605 & 1.619 & 1.385 & 1.176 & 0.959 & 0.730 & 0.501 & 0.302 & 0.158 & 0.069 & 0.023 & 0.006 & 0.001 & 0.000 & & \\
\hline 15 & 3119.70 & 3111.07 & 1.539 & 1.555 & 1.338 & 1.151 & 0.965 & 0.767 & 0.563 & 0.370 & 0.216 & 0.108 & 0.045 & 0.014 & 0.003 & 0.000 & 0.000 & \\
\hline 16 & 3327.21 & 3318.48 & 1.474 & 1.499 & 1.296 & 1.124 & 0.961 & 0.790 & 0.610 & 0.431 & 0.274 & 0.154 & 0.074 & 0.029 & 0.009 & 0.002 & 0.000 & 0.000 \\
\hline
\end{tabular}


Table 38. Calculated alpha factor mean values for CCF events in the GO cause group from 1997 through 2015.

\begin{tabular}{|c|c|c|c|c|c|c|c|c|c|c|c|c|c|c|c|c|}
\hline $\begin{array}{c}\text { Group } \\
\text { Size }\end{array}$ & $\alpha_{1}$ & $\alpha_{2}$ & $\alpha_{3}$ & $\alpha_{4}$ & $\alpha_{5}$ & $\alpha_{6}$ & $\alpha_{7}$ & $\alpha_{8}$ & $\alpha_{9}$ & $\alpha_{10}$ & $\alpha_{11}$ & $\alpha_{12}$ & $\alpha_{13}$ & $\alpha_{14}$ & $\alpha_{15}$ & $\alpha_{16}$ \\
\hline 2 & 0.9955 & $4.524 \mathrm{E}-03$ & & & & & & & & & & & & & & \\
\hline 3 & 0.9957 & $2.878 \mathrm{E}-03$ & $1.395 \mathrm{E}-03$ & & & & & & & & & & & & & \\
\hline 4 & 0.9956 & $3.196 \mathrm{E}-03$ & $5.458 \mathrm{E}-04$ & $6.209 \mathrm{E}-04$ & & & & & & & & & & & & \\
\hline 5 & 0.9962 & $2.588 \mathrm{E}-03$ & $9.651 \mathrm{E}-04$ & $2.110 \mathrm{E}-04$ & $3.024 \mathrm{E}-05$ & & & & & & & & & & & \\
\hline 6 & 0.9961 & $2.300 \mathrm{E}-03$ & 9.914E-04 & $3.968 \mathrm{E}-04$ & 9.108E-05 & 1.144E-04 & & & & & & & & & & \\
\hline 7 & 0.9965 & $1.776 \mathrm{E}-03$ & $1.025 \mathrm{E}-03$ & $5.066 \mathrm{E}-04$ & $1.795 \mathrm{E}-04$ & $4.195 \mathrm{E}-05$ & 5.361E-06 & & & & & & & & & \\
\hline 8 & 0.9965 & $1.442 \mathrm{E}-03$ & $9.480 \mathrm{E}-04$ & $5.638 \mathrm{E}-04$ & $2.600 \mathrm{E}-04$ & $8.731 \mathrm{E}-05$ & $2.002 \mathrm{E}-05$ & $1.367 \mathrm{E}-04$ & & & & & & & & \\
\hline 9 & 0.9969 & $1.167 \mathrm{E}-03$ & 8.572E-04 & $5.743 \mathrm{E}-04$ & 3.184E-04 & $1.377 \mathrm{E}-04$ & 4.460E-05 & 9.751E-06 & $1.042 \mathrm{E}-06$ & & & & & & & \\
\hline 10 & 0.9971 & $9.702 \mathrm{E}-04$ & 7.612E-04 & $5.556 \mathrm{E}-04$ & $3.516 \mathrm{E}-04$ & $1.828 \mathrm{E}-04$ & $7.538 \mathrm{E}-05$ & $2.344 \mathrm{E}-05$ & $4.801 \mathrm{E}-06$ & 4.691E-07 & & & & & & \\
\hline 11 & 0.9972 & $8.244 \mathrm{E}-04$ & $6.728 \mathrm{E}-04$ & $5.217 \mathrm{E}-04$ & $3.635 \mathrm{E}-04$ & $2.169 \mathrm{E}-04$ & $1.070 \mathrm{E}-04$ & 4.234E-05 & $1.250 \mathrm{E}-05$ & $2.379 \mathrm{E}-06$ & $2.133 \mathrm{E}-07$ & & & & & \\
\hline 12 & 0.9974 & 7.132E-04 & $5.960 \mathrm{E}-04$ & $4.820 \mathrm{E}-04$ & 3.602E-04 & $2.386 \mathrm{E}-04$ & $1.351 \mathrm{E}-04$ & 6.386E-05 & $2.416 \mathrm{E}-05$ & $6.708 \mathrm{E}-06$ & $1.183 \mathrm{E}-06$ & 9.777E-08 & & & & \\
\hline 13 & 0.9975 & $6.260 \mathrm{E}-04$ & $5.306 \mathrm{E}-04$ & $4.418 \mathrm{E}-04$ & $3.474 \mathrm{E}-04$ & $2.489 \mathrm{E}-04$ & $1.570 \mathrm{E}-04$ & $8.510 \mathrm{E}-05$ & $3.863 \mathrm{E}-05$ & $1.388 \mathrm{E}-05$ & $3.605 \mathrm{E}-06$ & 5.899E-07 & $4.513 \mathrm{E}-08$ & & & \\
\hline 14 & 0.9976 & $5.560 \mathrm{E}-04$ & 4.754E-04 & 4.036E-04 & 3.294E-04 & $2.506 \mathrm{E}-04$ & $1.719 \mathrm{E}-04$ & $1.038 \mathrm{E}-04$ & $5.420 \mathrm{E}-05$ & $2.354 \mathrm{E}-05$ & 7.981E-06 & $1.935 \mathrm{E}-06$ & $2.944 \mathrm{E}-07$ & $2.096 \mathrm{E}-08$ & & \\
\hline 15 & 0.9977 & 4.986E-04 & $4.288 \mathrm{E}-04$ & $3.689 \mathrm{E}-04$ & 3.093E-04 & $2.460 \mathrm{E}-04$ & $1.803 \mathrm{E}-04$ & 1.187E-04 & $6.917 \mathrm{E}-05$ & $3.477 \mathrm{E}-05$ & $1.437 \mathrm{E}-05$ & $4.578 \mathrm{E}-06$ & $1.036 \mathrm{E}-06$ & $1.470 \mathrm{E}-07$ & $0.000 \mathrm{E}+00$ & \\
\hline 16 & 0.9978 & 4.506E-04 & 3.894E-04 & $3.378 \mathrm{E}-04$ & $2.888 \mathrm{E}-04$ & $2.375 \mathrm{E}-04$ & $1.834 \mathrm{E}-04$ & $1.294 \mathrm{E}-04$ & $8.230 \mathrm{E}-05$ & $4.638 \mathrm{E}-05$ & $2.235 \mathrm{E}-05$ & 8.743E-06 & $2.614 \mathrm{E}-06$ & 5.534E-07 & 7.347E-08 & $0.000 \mathrm{E}+00$ \\
\hline
\end{tabular}

Table 39. Estimated CCF industry-wide prior distributions with CCF events in the GO cause group from 1997 through 2015.

\begin{tabular}{|c|c|c|c|c|c|c|c|c|c|c|c|c|c|c|c|c|}
\hline $\begin{array}{c}\text { Group } \\
\text { Size } \\
\end{array}$ & $\alpha_{1}$ & $\mathrm{~b}_{1}$ & $\alpha_{2}$ & $\mathrm{~b}_{2}$ & $\alpha_{3}$ & $\mathrm{~b}_{3}$ & $\alpha_{4}$ & $\mathrm{~b}_{4}$ & $\alpha_{5}$ & $b_{5}$ & $\alpha_{6}$ & $\mathrm{~b}_{6}$ & $\alpha_{7}$ & $\mathrm{~b}_{7}$ & $\alpha_{8}$ & $\mathrm{~b}_{8}$ \\
\hline 2 & $1.085 \mathrm{E}+02$ & $4.932 \mathrm{E}-01$ & 4.932E-01 & $1.085 \mathrm{E}+02$ & & & & & & & & & & & & \\
\hline 3 & $2.469 \mathrm{E}+02$ & $1.060 \mathrm{E}+00$ & 7.137E-01 & $2.473 \mathrm{E}+02$ & $3.458 \mathrm{E}-01$ & $2.476 \mathrm{E}+02$ & & & & & & & & & & \\
\hline 4 & $4.827 \mathrm{E}+02$ & $2.115 \mathrm{E}+00$ & $1.549 \mathrm{E}+00$ & $4.832 \mathrm{E}+02$ & $2.646 \mathrm{E}-01$ & $4.845 \mathrm{E}+02$ & $3.010 \mathrm{E}-01$ & $4.845 \mathrm{E}+02$ & & & & & & & & \\
\hline 5 & $1.398 \mathrm{E}+03$ & $|5.325 \mathrm{E}+00|$ & $3.632 \mathrm{E}+00$ & $1.400 \mathrm{E}+03$ & $1.354 \mathrm{E}+00$ & $1.402 \mathrm{E}+03$ & $2.961 \mathrm{E}-01$ & $1.403 E+03$ & $4.243 \mathrm{E}-02$ & $1.403 \mathrm{E}+03$ & & & & & & \\
\hline 6 & $1.263 \mathrm{E}+03$ & $4.936 \mathrm{E}+00$ & $2.915 \mathrm{E}+00$ & $1.265 \mathrm{E}+03$ & $1.257 \mathrm{E}+00$ & $1.267 \mathrm{E}+03$ & 5.031E- 01 & $1.267 \mathrm{E}+03$ & $1.155 \mathrm{E}-01$ & $1.268 \mathrm{E}+03$ & $1.451 \mathrm{E}-01$ & $1.268 \mathrm{E}+03$ & & & & \\
\hline 7 & $2.722 \mathrm{E}+03$ & $|9.655 \mathrm{E}+00|$ & $4.851 \mathrm{E}+00$ & $2.727 \mathrm{E}+03$ & $2.800 \mathrm{E}+00$ & $2.729 \mathrm{E}+03$ & $1.384 \mathrm{E}+00$ & $2.731 \mathrm{E}+03$ & $4.902 \mathrm{E}-01$ & $2.731 \mathrm{E}+03$ & $1.146 \mathrm{E}-01$ & $2.732 \mathrm{E}+03$ & $1.464 \mathrm{E}-02$ & $2.732 \mathrm{E}+03$ & & \\
\hline 8 & $2.061 \mathrm{E}+03$ & $7.148 \mathrm{E}+00$ & $2.980 \mathrm{E}+00$ & $2.065 \mathrm{E}+03$ & $1.960 \mathrm{E}+00$ & $2.066 \mathrm{E}+03$ & $1.166 \mathrm{E}+00$ & $2.067 \mathrm{E}+03$ & $5.375 \mathrm{E}-01$ & $2.067 \mathrm{E}+03$ & $1.805 \mathrm{E}-01$ & $2.068 \mathrm{E}+03 \mid$ & $4.139 \mathrm{E}-02$ & $2.068 \mathrm{E}+03$ & $2.827 \mathrm{E}-01$ & $2.068 \mathrm{E}+03$ \\
\hline 9 & $4.897 \mathrm{E}+03$ & $1.528 \mathrm{E}+01$ & $5.733 \mathrm{E}+00$ & $4.907 \mathrm{E}+03$ & $4.211 \mathrm{E}+00$ & $4.909 \mathrm{E}+03$ & $2.821 \mathrm{E}+00$ & $4.910 \mathrm{E}+03$ & $1.564 \mathrm{E}+00$ & $4.911 \mathrm{E}+03$ & $6.763 \mathrm{E}-01$ & $4.912 \mathrm{E}+03$ & 2.191E-01 & $4.913 \mathrm{E}+03$ & $4.790 \mathrm{E}-02$ & $4.913 \mathrm{E}+03$ \\
\hline 10 & $6.399 \mathrm{E}+03$ & $1.877 \mathrm{E}+01$ & $6.226 \mathrm{E}+00$ & $6.411 \mathrm{E}+03$ & $4.884 \mathrm{E}+00$ & $6.413 \mathrm{E}+03$ & $3.565 \mathrm{E}+00$ & $6.414 \mathrm{E}+03$ & $2.256 \mathrm{E}+00$ & $6.415 \mathrm{E}+03$ & $1.173 \mathrm{E}+00$ & $6.416 \mathrm{E}+03$ & 4.837E-01 & $6.417 \mathrm{E}+03$ & $1.504 \mathrm{E}-01$ & $6.417 \mathrm{E}+0$ \\
\hline
\end{tabular}




\begin{tabular}{|c|c|c|c|c|c|c|c|c|c|c|c|c|c|c|c|c|}
\hline $\begin{array}{c}\text { Group } \\
\text { Size }\end{array}$ & $\alpha_{1}$ & $\mathrm{~b}_{1}$ & $\alpha_{2}$ & $b_{2}$ & $\alpha_{3}$ & $\mathrm{~b}_{3}$ & $\alpha_{4}$ & $\mathrm{~b}_{4}$ & $\alpha_{5}$ & $\mathrm{~b}_{5}$ & $\alpha_{6}$ & $\mathrm{~b}_{6}$ & $\alpha_{7}$ & $b_{7}$ & $\alpha_{8}$ & $\mathrm{~b}_{8}$ \\
\hline 11 & $237 \mathrm{E}+03$ & $2.283 \mathrm{E}+01$ & $6.809 \mathrm{E}+00$ & $8.253 \mathrm{E}+03$ & $5.557 \mathrm{E}+00$ & $8.254 \mathrm{E}+03$ & $4.309 \mathrm{E}+00$ & $8.255 \mathrm{E}+03$ & $3.002 \mathrm{E}+00$ & $8.257 \mathrm{E}+03$ & $1.792 \mathrm{E}+00$ & $8.258 \mathrm{E}+03$ & $8.840 \mathrm{E}-01$ & $8.259 \mathrm{E}+03$ & $3.497 \mathrm{E}-01$ & $8.259 \mathrm{E}+03$ \\
\hline 12 & $1.047 \mathrm{E}+04$ & $2.751 \mathrm{E}+01$ & $7.485 \mathrm{E}+00$ & $1.049 \mathrm{E}+04$ & $6.255 \mathrm{E}+00$ & $1.049 \mathrm{E}+04$ & $5.059 \mathrm{E}+00$ & $1.049 \mathrm{E}+04$ & $3.780 \mathrm{E}+00$ & $1.049 \mathrm{E}+04$ & $2.504 \mathrm{E}+00$ & $1.049 \mathrm{E}+04$ & $1.418 \mathrm{E}+00$ & $1.050 \mathrm{E}+04$ & 6.702 & $1.050 \mathrm{E}+04$ \\
\hline 13 & $.316 \mathrm{E}+04$ & $3.289 \mathrm{E}+01$ & $8.258 \mathrm{E}+00$ & $1.319 \mathrm{E}+04$ & $6.999 \mathrm{E}+00$ & $1.319 \mathrm{E}+04$ & $5.828 \mathrm{E}+00$ & $1.319 \mathrm{E}+04$ & $4.583 \mathrm{E}+00$ & $1.319 \mathrm{E}+04$ & $3.284 \mathrm{E}+00$ & $1.319 \mathrm{E}+04$ & $2.071 \mathrm{E}+00$ & $1.319 \mathrm{E}+04$ & $1.123 \mathrm{E}+00$ & $1.319 \mathrm{E}+04$ \\
\hline 14 & $1.639 \mathrm{E}+04$ & $3.908 \mathrm{E}+01$ & $9.133 \mathrm{E}+00$ & $1.642 \mathrm{E}+04$ & $7.810 \mathrm{E}+00$ & $1.642 \mathrm{E}+04$ & $6.631 \mathrm{E}+00$ & $1.642 \mathrm{E}+04$ & $5.411 \mathrm{E}+00$ & $1.642 \mathrm{E}+04$ & $4.116 \mathrm{E}+00$ & $1.643 \mathrm{E}+04$ & $2.823 \mathrm{E}+00$ & $1.643 \mathrm{E}+04$ & $1.705 \mathrm{E}+00$ & $1.643 \mathrm{E}+04$ \\
\hline 15 & $2.192 \mathrm{E}+04$ & $4.998 \mathrm{E}+01$ & $1.095 \mathrm{E}+01$ & $2.196 \mathrm{E}+04$ & $9.422 \mathrm{E}+00$ & $2.196 \mathrm{E}+04$ & $8.105 \mathrm{E}+00$ & $2.197 \mathrm{E}+04$ & $6.795 \mathrm{E}+00$ & $2.197 \mathrm{E}+04$ & $5.405 \mathrm{E}+00$ & $2.197 \mathrm{E}+04$ & $3.962 \mathrm{E}+00$ & $2.197 \mathrm{E}+04$ & $2.608 \mathrm{E}+00$ & $2.197 \mathrm{E}+04$ \\
\hline 16 & $2.553 \mathrm{E}+04$ & $5.578 \mathrm{E}+01$ & $1.153 \mathrm{E}+01$ & $2.558 \mathrm{E}+04$ & $9.963 \mathrm{E}+00$ & $2.558 \mathrm{E}+04$ & $8.644 \mathrm{E}+00$ & $2.558 \mathrm{E}+04$ & $7.391 \mathrm{E}+00$ & $2.558 \mathrm{E}+04$ & $6.077 \mathrm{E}+00$ & $2.558 \mathrm{E}+04$ & $4.692 \mathrm{E}+00$ & $2.559 \mathrm{E}+04$ & $3.312 \mathrm{E}+00$ & $2.559 \mathrm{E}+04$ \\
\hline
\end{tabular}

\begin{tabular}{|c|c|c|c|c|c|c|c|c|c|c|c|c|c|c|c|c|}
\hline $\begin{array}{c}\text { Group } \\
\text { Size } \\
\end{array}$ & $\alpha_{9}$ & $b_{9}$ & $\alpha_{10}$ & $b_{10}$ & $\alpha_{11}$ & $b_{11}$ & $\alpha_{12}$ & $b_{12}$ & $\alpha_{13}$ & $b_{13}$ & $\alpha_{14}$ & $b_{14}$ & $\alpha_{15}$ & $b_{15}$ & $\alpha_{16}$ & $b_{16}$ \\
\hline 9 & 5.119E-03 & $4.913 \mathrm{E}+03$ & & & & & & & & & & & & & & \\
\hline 10 & $3.081 \mathrm{E}-02$ & $6.418 \mathrm{E}+03$ & $3.010 \mathrm{E}-03$ & $6.418 \mathrm{E}+03$ & & & & & & & & & & & & \\
\hline 11 & $1.032 \mathrm{E}-01$ & $8.259 \mathrm{E}+03$ & $1.965 \mathrm{E}-02$ & $8.260 \mathrm{E}+03$ & $1.761 \mathrm{E}-03$ & $8.260 \mathrm{E}+03$ & & & & & & & & & & \\
\hline 12 & $2.535 \mathrm{E}-01$ & $1.050 \mathrm{E}+04$ & 7.040E-02 & $1.050 \mathrm{E}+04$ & $1.242 \mathrm{E}-02$ & $1.050 \mathrm{E}+04$ & $1.026 \mathrm{E}-03$ & $1.050 \mathrm{E}+04$ & & & & & & & & \\
\hline 13 & $5.097 \mathrm{E}-01$ & $1.319 \mathrm{E}+04$ & $1.831 \mathrm{E}-01$ & $1.319 \mathrm{E}+04$ & $4.756 \mathrm{E}-02$ & $1.319 \mathrm{E}+04$ & 7.782E-03 & $1.319 \mathrm{E}+04$ & 5.954E-04 & $1.319 \mathrm{E}+04$ & & & & & & \\
\hline 14 & 8.904E-01 & $1.643 \mathrm{E}+04$ & 3.868E-01 & $1.643 \mathrm{E}+04$ & $1.311 \mathrm{E}-01$ & $1.643 \mathrm{E}+04$ & 3.179E-02 & $1.643 \mathrm{E}+04$ & 4.836E-03 & $1.643 \mathrm{E}+04$ & $3.443 \mathrm{E}-04$ & $1.643 \mathrm{E}+04$ & & & & \\
\hline 15 & $1.520 \mathrm{E}+00$ & $2.197 \mathrm{E}+04$ & 7.638E-01 & $2.197 \mathrm{E}+04$ & 3.157E-01 & $2.197 \mathrm{E}+04$ & $1.006 \mathrm{E}-01$ & $2.197 \mathrm{E}+04$ & 2.277E-02 & $2.197 \mathrm{E}+04$ & $3.231 \mathrm{E}-03$ & $2.197 \mathrm{E}+04$ & 7.043E-05 & $2.197 \mathrm{E}+04$ & & \\
\hline 16 & $2.106 \mathrm{E}+00$ & $2.559 \mathrm{E}+04$ & $1.187 \mathrm{E}+00$ & $2.559 \mathrm{E}+04$ & 5.718E-01 & $2.559 \mathrm{E}+04$ & 2.237E-01 & $2.559 \mathrm{E}+04$ & $6.688 \mathrm{E}-02$ & $2.559 \mathrm{E}+04$ & $1.416 \mathrm{E}-02$ & $2.559 \mathrm{E}+04$ & $1.880 \mathrm{E}-03$ & $2.559 \mathrm{E}+04$ & $7.690 \mathrm{E}-05$ & $2.559 \mathrm{E}+04$ \\
\hline
\end{tabular}




\section{VARIOUS ITEMS ON PRIOR DISTRIBUTION DEVELOPMENT AND CCF ANALYSIS}

While this report follows the existing process of updating (or developing) generic prior distributions for CCF alpha factors (or causal alpha factors), certain issues were noted and discussed during the study, including whether the impact vector and mapping method is appropriate for use in prior distribution development, and whether better methodologies are available. The following is a list of such items, along with preliminary thoughts on them.

\section{Is the impact vector and mapping method really appropriate for CCF parameter estimations or prior determination? Are there any other alternative approaches for developing the prior distributions?}

The impact vector and mapping method was introduced in NUREG/CR-4780, published in 1988. To obtain a high degree of consensus on the principles of treating CCF in risk analysis, the report was reviewed by many experts and organizations in the U.S. and Europe. Appendix D of NUREG/CR4780 provides a detailed discussion on the background and justification for using the mapping method in parameter estimation. While mapping up and mapping down does introduce greater uncertainties in CCF parameter estimation, the method seems reasonable for use in treating scarce CCF data and estimating associated CCF parameters. Actually, the impact vector and mapping method was used consistently in the subsequent NRC CCF studies, becoming the state-of-the-art in CCF event characterization and CCF parameter estimation.

On the other hand, one could always look for alternative approaches that may be better for CCF parameter estimation or prior distribution development. After nearly 30 years of using the approach, it may be worthwhile to survey what other methodologies may be available, enabling a determination as to whether any alternative approaches exist that are better suited for prior distribution development. It should be noted that regardless of the method selected, there will always be uncertainty related to it. At minimum, certain kinds of sensitivity studies could be conducted to compare the mapping method with other methods of obtaining prior distributions. These sensitivity studies could then be evaluated to determine the potential impact of different methodologies on the associated risk applications (e.g., the significance determination process).

2. What is the actual process for developing prior distributions for CCF parameters? Is this process defensible? Are there any issues involved?

While NUREG/CR-5485 provides a brief description of several practical approaches to developing prior distributions for CCF parameters, no published papers describe the actual process. One objective of this report is to document the existing process of developing prior distributions for $\mathrm{CCF}$ parameters. To engage outside experts for their insights, other efforts (e.g., publishing the process in national and international conferences/journals and on the NRC website) are considered.

The impact vector and mapping method has been used consistently in the NRC CCF studies, becoming the state-of-the-art in CCF event characterization and CCF parameter estimation. However, additional uncertainties are associated with this approach. Sensitivity analysis should be performed to understand the impact associated with this model uncertainty.

During the study, we also encountered several issues involving the details of the existing process (e.g., the binomial regression treatment for complete CCF events, estimation of the $\rho$ parameter associated with the mapping up methodology, and the question of whether different prior distributions should be developed for different component types). These specific issues are discussed in more detail below. 


\section{Is using the binomial regression treatment for complete CCF events in the current prior development process appropriate? What should be the proper function for use in curve fitting?}

The background on why a different treatment was used for complete CCF events in the prior distribution development process is unclear, but it appears to be a compromise addressing the concern that the mapping technique adds too many pseudo-CCF events into the data analysis. As to whether the treatment is appropriate, and which function should be used for curve fitting, these may be good topics for further discussion. For example:

a) When using MatLab and Eq. 5 to curve fit the CCF data for the GE cause group from 1997 through 2015, negative values would be obtained for the probability of complete CCF event in the larger group sizes.

b) For causal CCF data, many group sizes feature zero complete CCF events, or even zero total CCF events. This leads to less valid data points for a good curve fitting.

c) The variables used in curve fitting complete CCF events are group size and probability of complete CCF event. The estimated number of complete CCF events is obtained by multiplying the probability of complete CCF event by the total number of CCF events. With the larger values in the total number of CCF events for some group sizes (e.g., group sizes 12 and 16), the estimated number of complete CCF events is no longer a smooth curve for some group sizes but appears as spikes.

d) The current binomial regression treatment of complete CCF events does not distinguish lethal shock events from nonlethal shock but complete CCF events. For lethal shocks, the impact vector should be mapped directly (i.e., the probability that all $x$ components in a system of $x$ components have failed due to a lethal shock is mapped directly and equals the probability of failing all $y$ components in a system of $y$ components). The correct process should treat lethal shock events and non-lethal shock but complete CCF events differently: mapping the lethal shock events directly, while curve fitting the nonlethal shock but complete CCF events.

e) Fortunately, the lethal event issue should have little impact on the results, as a review of the CCF data used in this study (1997-2015) only found three CCF events coded as have been induced by lethal shock.

4. How is the mapping up factor $\rho$ determined in the current process? Is there a better way to estimate $\rho ?$

The mapping up factor $\rho$ is a very important parameter in the mapping methodology, as it is included in the mapping up formula and would greatly impact the mapping results, depending on its assumed value. The parameter $\rho$ is defined in the Binomial Failure Rate (BFR) model as the conditional probability of failure of each component, given a nonlethal shock. For example, consider the following formula, used to map a system of group size 2 up to a system of group size 3 or 4 :

$$
\begin{gathered}
P_{3}^{(3)}=\rho P_{2}^{(2)} \\
P_{4}^{(4)}=\rho^{2} P_{2}^{(2)}
\end{gathered}
$$

where $P_{x}^{(x)}$ is the probability of all $x$ components failing in a system of $x$ components. Depending on the assumed value of $\rho$, the mapped up results of $P_{3}^{(3)}$ and $P_{4}^{(4)}$ would change significantly for the same $P_{2}^{(2)}$ ( 1 or 0.5 in the example below): 
Table 40. Impact of mapping up factor.

\begin{tabular}{|c|c|c|c|c|c|c|c|c|}
\hline & \multicolumn{4}{|c|}{$P_{2}{ }^{(2)}=1$} & \multicolumn{4}{c|}{$P_{2}{ }^{(2)}=0.5$} \\
\hline & $\rho=1$ & $\rho=0.8$ & $\rho=0.5$ & $\rho=0.2$ & $\rho=1$ & $\rho=0.8$ & $\rho=0.5$ & $\rho=0.2$ \\
\hline$P_{3}^{(3)}$ & 1 & 0.8 & 0.5 & 0.2 & 0.5 & 0.4 & 0.25 & 0.1 \\
\hline$P_{4}^{(4)}$ & 1 & 0.64 & 0.25 & 0.04 & 0.5 & 0.32 & 0.125 & 0.02 \\
\hline
\end{tabular}

NUREG/CR-6268 Rev.1 provides a method of estimating the mapping up factor $\rho$ via the following equation:

$$
\begin{aligned}
& \rho=\frac{\sum_{i=1}^{m} i(i-1) f_{i}}{(m-1) \sum_{i=1}^{m} i\left(f_{i}\right)} \\
& \text { where } \\
& \begin{array}{l}
m= \\
\text { the number of elements in the group } \\
f_{i}=
\end{array} \\
& \text { the } i^{\text {th }} \text { element of the generic impact } \\
& \text { vector. }
\end{aligned}
$$

(Eq. 8)

where a maximum value of 0.85 is established based on observed trends and empirical studies. In the CCF Parameter Estimations 2003 Update, the previously recommended value of 0.85 was considered too conservative, so 0.50 was recommended and has been used ever since.

Appendix $\mathrm{C}$ develops a process for estimating the mapping up factor $\rho$. This process underwent preliminarily testing using the pump CCF data. Whether this new process to estimate $\rho$ should be applied to prior development is subject to further review and decision.

\section{Should different prior distributions be developed for different component groups?}

With some simple examinations of the CCF data, Atwood suggests that (a) different component types have quite different alpha factors and should thus be analyzed separately; and (b) if the different failure causes are to be considered, the analysis must distinguish among component types, as various components have different susceptibilities to the different failure causes.

\section{Is there a general formula for mapping up CCF data?}

A table of formulas is presented in NUREG/CR4780 (Table D-5) and NUREG/CR-5485 (Table C-5) for mapping up events classified as nonlethal shocks. This table is expanded in NUREG/CR-6268, Rev. 1 (Table 7-4), with the maximum size of system mapping to being increased from 4 to 6. 
Table 7-4. Formulas for upward mapping of events classified as non-lethal shocks.

\begin{tabular}{|c|c|c|c|c|c|c|}
\hline \multicolumn{7}{|c|}{ Size of System Mapping To (Number of Identical Trains) } \\
\hline \multirow{6}{*}{ 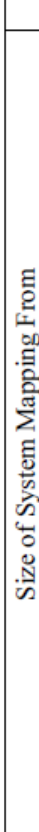 } & & 2 & 3 & 4 & 5 & 6 \\
\hline & 1 & $\begin{array}{l}\mathrm{f}_{1}^{(2)}=2(1-\rho) \mathrm{f}_{1}^{(1)} \\
\mathrm{f}_{2}^{(2)}=\rho \mathrm{f}_{1}^{(1)}\end{array}$ & $\begin{array}{l}f_{1}^{(3)}=3(1-\rho)^{2} f_{1}^{(1)} \\
f_{2}^{(3)}=3 \rho(1-\rho) f_{1}^{(1)} \\
f_{3}^{(3)}=\rho^{2} f_{1}^{(1)}\end{array}$ & $\begin{array}{l}\mathrm{f}_{1}^{(4)}=4(1-\rho)^{3} \mathrm{f}_{1}^{(1)} \\
\mathrm{f}_{2}^{(4)}=6 \rho(1-\rho)^{2} \mathrm{f}_{1}^{(1)} \\
\mathrm{f}_{3}^{(4)}=4 \rho^{2}(1-\rho) \mathrm{f}_{1}^{(1)} \\
\mathrm{f}_{4}^{(4)}=\rho^{3} \mathrm{f}_{1}^{(1)}\end{array}$ & $\begin{array}{l}\mathrm{f}_{1}^{(5)}=5(1-\rho)^{4} \mathrm{f}_{1}^{(1)} \\
\mathrm{f}_{2}^{(5)}=10 \rho(1-\rho)^{3} \mathrm{f}_{1}^{(1)} \\
\mathrm{f}_{3}^{(5)}=10 \rho^{2}(1-\rho)^{2} \mathrm{f}_{1}^{(1)} \\
\mathrm{f}_{4}^{(5)}=5 \rho^{3}(1-\rho) \mathrm{f}_{1}^{(1)} \\
\mathrm{f}_{5}^{(5)}=\rho^{4} \mathrm{f}_{1}(1)\end{array}$ & 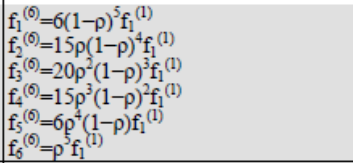 \\
\hline & 2 & & $\begin{array}{l}\mathrm{f}_{1}^{(3)}=(3 / 2)(1-\rho) \mathrm{f}_{1}{ }^{(2)} \\
\mathrm{f}_{2}^{(3)}=\rho \mathrm{f}_{1}(2)+(1-\rho) \mathrm{f}_{2}{ }^{(2)} \\
\mathrm{f}_{3}{ }^{(3)}=\rho \mathrm{f}_{2}(2)\end{array}$ & $\begin{array}{l}\mathrm{f}_{1}{ }^{(4)}=2(1-\rho)^{2} \mathrm{f}_{1}^{(2)} \\
\mathrm{f}_{2}{ }^{(4)}=(5 / 2) \rho(1-\rho) \mathrm{f}_{1}{ }^{(2)}+(1-\rho)^{2} \mathrm{f}_{2}{ }^{(2)} \\
\mathrm{f}_{3}{ }^{(4)}=\rho^{2} \mathrm{f}_{1}(2)+2 \rho(1-\rho) \mathrm{f}_{2}^{(2)} \\
\mathrm{f}_{4}^{(4)}=\rho^{2} \mathrm{f}_{2}{ }^{(2)}\end{array}$ & $\begin{array}{l}\mathrm{f}_{1}^{(5)}=(5 / 2)(1-\rho)^{3} \mathrm{f}_{1}^{(2)} \\
\mathrm{f}_{2}^{(5)}=(9 / 2) \rho(1-\rho)^{2} \mathrm{f}_{1}{ }^{(2)}+(1-\rho)^{3} \mathrm{f}_{2}{ }^{(2)} \\
\mathrm{f}_{3}^{(5)}=(7 / 2) \rho^{2}(1-\rho) \mathrm{f}_{1}^{(2)}+3 \rho(1-\rho)^{2} \mathrm{f}_{2}^{(2)} \\
\mathrm{f}_{4}^{(5)}=\rho^{3} \mathrm{f}_{1}^{(2)}+3 \rho^{2}(1-\rho) \mathrm{f}_{2}^{(2)} \\
\mathrm{f}_{5}^{(5)}=\rho^{3} \mathrm{f}_{2}^{(2)}\end{array}$ & 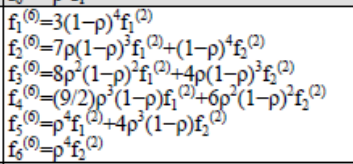 \\
\hline & 3 & & & $\begin{array}{l}\mathrm{f}_{1}{ }^{(4)}=(4 / 3)(1-\rho) \mathrm{f}_{1}{ }^{(3)} \\
\mathrm{f}_{2}{ }^{(4)}=\rho \mathrm{f}_{1}{ }_{1}^{(3)}+(1-\rho) \mathrm{f}_{2}^{(3)} \\
\mathrm{f}_{3}^{(4)}=\rho \mathrm{f}_{2}^{(3)}+(1-\rho) \mathrm{f}_{3}{ }^{(3)} \\
\mathrm{f}_{4}{ }^{(4)}=\rho \mathrm{f}_{3}{ }^{(3)}\end{array}$ & $\begin{array}{l}\mathrm{f}_{1}^{(5)}=(5 / 3)(1-\rho)^{2} \mathrm{f}_{1}^{(3)} \\
\mathrm{f}_{2}^{(5)}=(7 / 3) \rho(1-\rho) \mathrm{f}_{1}^{(3)}+(1-\rho)^{2} \mathrm{f}_{2}^{(3)} \\
\mathrm{f}_{3}^{(5)}=\rho^{2} \mathrm{f}_{1}^{(3)}+2 \rho(1-\rho) \mathrm{f}_{2}^{(3)}+(1-\rho)^{2} \mathrm{f}_{3}^{(3)} \\
\mathrm{f}_{4}^{(5)}=\rho^{2} \mathrm{f}_{2}{ }^{(3)}+2 \rho(1-\rho) \mathrm{f}_{3}^{(3)} \\
\mathrm{f}_{5}^{(5)}=\rho^{2} \mathrm{f}_{3}^{(3)}\end{array}$ & 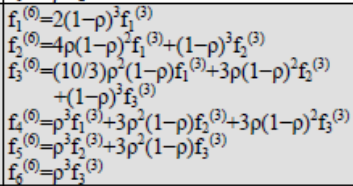 \\
\hline & 4 & & & & $\begin{array}{l}\mathrm{f}_{1}^{(5)}=(5 / 4)(1-\rho) \mathrm{f}_{1}^{(4)} \\
\mathrm{f}_{2}^{(5)}=\rho \mathrm{f}_{1}^{(4)}+(1-\rho) \mathrm{f}_{2}{ }^{(4)} \\
\mathrm{f}_{3}^{(5)}=\rho \mathrm{f}_{2}{ }^{(4)}+(1-\rho) \mathrm{f}_{3}^{(4)} \\
\mathrm{f}_{4}^{(5)}=\rho \mathrm{f}_{3}^{(4)}+(1-\rho) \mathrm{f}_{4}^{(4)} \\
\mathrm{f}_{5}^{(5)}=\rho \mathrm{f}_{4}^{(4)}\end{array}$ & 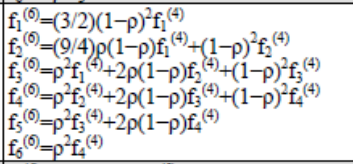 \\
\hline & 5 & & & & & 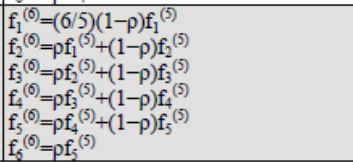 \\
\hline
\end{tabular}

Appendix D provides explicit justification - as well as an explicit general formula — for the mapping up method. The following proposed general equation for calculating the mapping up formulas is compared with the above NUREG/CR6268 table:

$$
n_{K}{ }^{(M)}=\frac{\left(\begin{array}{c}
M \\
K
\end{array}\right)}{\left(\begin{array}{c}
M \\
K
\end{array}\right)-\left(\begin{array}{c}
M-m \\
K
\end{array}\right)}\left[\sum_{k}\left(\begin{array}{c}
M-m \\
K-k
\end{array}\right) \rho^{K-k}(1-\rho)^{(M-m)-(K-k)} n_{k}^{(m)}\right]
$$

Several differences are found, as seen in the following table. For example, the NUREG table includes the following formula for mapping a system of size 2 to a system of size 4 :

$$
f(2,4)=(5 / 2) \rho(1-\rho) f(1,2)+(1-\rho)^{\wedge} 2 f(2,2)
$$

whereas the general formula includes the following:

$$
f(2,4)=(12 / 5) \rho(1-\rho) f(1,2)+(6 / 5)(1-\rho)^{\wedge} 2 \mathrm{f}(2,2)
$$

Using the same deduction as found in Appendix C.4.3 of NUREG/CR-5485, we see that the $\mathrm{f}(2,4)$ equation derived from the general formula (Eq. 9) is actually correct. 


\begin{tabular}{|c|c|c|c|c|c|c|}
\hline \multirow{2}{*}{\multicolumn{2}{|c|}{$\begin{array}{l}\text { Mapping Up } \\
\text { Formulas }\end{array}$}} & \multicolumn{5}{|c|}{ Size of System Mapping To (Number of Identical Trains) } \\
\hline & & 2 & 3 & 4 & 5 & 6 \\
\hline \multirow{3}{*}{$\begin{array}{l}\text { Size of } \\
\text { System } \\
\text { Mapping } \\
\text { From }\end{array}$} & 1 & $\begin{array}{l}f(1,2)=2(1-\rho) \\
f(1,1) \\
f(2,2)=\rho \\
f(1,1)\end{array}$ & $\begin{array}{l}\mathrm{f}(1,3)=3(1-\rho)^{\wedge} 2 \\
\mathrm{f}(1,1) \\
\mathrm{f}(2,3)=3 \rho(1-\rho) \\
\mathrm{f}(1,1) \\
\mathrm{f}(3,3)=\rho^{\wedge} 2 \mathrm{f}(1,1)\end{array}$ & $\begin{array}{l}\mathrm{f}(1,4)=4(1-\rho)^{\wedge} 3 \mathrm{f}(1,1) \\
\mathrm{f}(2,4)=6 \rho(1-\rho)^{\wedge} 2 \mathrm{f}(1,1) \\
\mathrm{f}(3,4)=4 \rho^{\wedge} 2(1-\rho) \mathrm{f}(1,1) \\
\mathrm{f}(4,4)=\rho^{\wedge} 3 \mathrm{f}(1,1)\end{array}$ & $\begin{array}{l}\mathrm{f}(1,5)=5(1-\rho)^{\wedge} 4 \mathrm{f}(1,1) \\
\mathrm{f}(2,5)=10 \rho(1-\rho)^{\wedge} 3 \mathrm{f}(1,1) \\
\mathrm{f}(3,5)=10 \rho^{\wedge} 2(1-\rho)^{\wedge} 2 \mathrm{f}(1,1) \\
\mathrm{f}(4,5)=5 \rho^{\wedge} 3(1-\rho) \mathrm{f}(1,1) \\
\mathrm{f}(5,5)=\rho^{\wedge} 4 \mathrm{f}(1,1)\end{array}$ & $\begin{array}{l}\mathrm{f}(1,6)=6(1-\rho)^{\wedge} 5 \mathrm{f}(1,1) \\
\mathrm{f}(2,6)=15 \rho(1-\rho)^{\wedge} 4 \mathrm{f}(1,1) \\
\mathrm{f}(3,6)=20 \rho^{\wedge} 2(1-\rho)^{\wedge} 3 \mathrm{f}(1,1) \\
\mathrm{f}(4,6)=15 \rho^{\wedge} 3(1-\rho)^{\wedge} 2 \mathrm{f}(1,1) \\
\mathrm{f}(5,6)=6 \rho^{\wedge} 4(1-\rho) \mathrm{f}(1,1) \\
\mathrm{f}(6,6)=\rho^{\wedge} 5 \mathrm{f}(1,1)\end{array}$ \\
\hline & 2 & & $\begin{array}{l}\mathrm{f}(1,3)=(3 / 2)(1-\rho) \\
\mathrm{f}(1,2) \\
\mathrm{f}(2,3)=\rho \\
\mathrm{f}(1,2)+(1-\rho) \\
\mathrm{f}(2,2) \\
\mathrm{f}(3,3)=\rho \mathrm{f}(2,2)\end{array}$ & $\begin{array}{l}\mathrm{f}(1,4)=2(1-\rho)^{\wedge} 2 \mathrm{f}(1,2) \\
\mathrm{f}(2,4)=(5 / 2) \rho(1-\rho) \mathrm{f}(1,2)+(1-\rho)^{\wedge} 2 \mathrm{f}(2,2) \\
\mathrm{f}(2,4)=(\mathbf{1 2 / 5}) \rho(1-\rho) \mathrm{f}(1,2)+(\mathbf{6} / \mathbf{5})(1-\rho)^{\wedge} 2 \mathrm{f}(2,2) \\
\mathrm{f}(3,4)=\rho^{\wedge} 2 \mathrm{f}(1,2)+2 \rho(1-\rho) \mathrm{f}(2,2) \\
\mathrm{f}(4,4)=\rho^{\wedge} 2 \mathrm{f}(2,2)\end{array}$ & $\begin{array}{l}\mathrm{f}(1,5)=(5 / 2)(1-\rho)^{\wedge} 3 \mathrm{f}(1,2) \\
\mathrm{f}(2,5)=(9 / 2) \rho(1-\rho)^{\wedge} 2 \mathrm{f}(1,2)+(1-\rho)^{\wedge} 3 \\
\mathrm{f}(2,2) \\
\mathrm{f}(2,5)=(\mathbf{3 0} / 7) \rho(1-\rho)^{\wedge} 2 \\
\mathrm{f}(1,2)+(\mathbf{1 0 / 7})(1-\rho)^{\wedge} 3 \mathrm{f}(2,2) \\
\mathrm{f}(3,5)=(7 / 2) \rho^{\wedge} 2(1-\rho) \\
\mathrm{f}(1,2)+\mathbf{3} \rho(1-\rho)^{\wedge} 2 \mathrm{f}(2,2) \\
\mathrm{f}(3,5)=(\mathbf{1 0 / 3}) \rho^{\wedge} 2(1-\rho) \\
\mathrm{f}(1,2)+(\mathbf{1 0 / 3}) \rho(1-\rho)^{\wedge} 2 \mathrm{f}(2,2) \\
\mathrm{f}(4,5)=\rho^{\wedge} 3 \mathrm{f}(1,2)+3 \rho^{\wedge} 2(1-\rho) \mathrm{f}(2,2) \\
\mathrm{f}(5,5)=\rho^{\wedge} 3 \mathrm{f}(2,2)\end{array}$ & $\begin{array}{l}\mathrm{f}(1,6)=3(1-\rho)^{\wedge} 4 \mathrm{f}(1,2) \\
\mathrm{f}(2,6)=7 \rho(1-\rho)^{\wedge} 3 \mathrm{f}(1,2)+(1-\rho)^{\wedge} 4 \mathrm{f}(2,2) \\
\mathrm{f}(2,6)=(\mathbf{2 0 / 3}) \rho(1-\rho)^{\wedge} 3 \mathrm{f}(1,2)+(\mathbf{5} / 3)(1-\rho)^{\wedge} 4 \mathrm{f}(2,2) \\
\mathrm{f}(3,6)=\mathbf{8} \rho^{\wedge} 2(1-\rho)^{\wedge} 2 \mathrm{f}(1,2)+4 \rho(1-\rho)^{\wedge} 3 \mathrm{f}(2,2) \\
\mathrm{f}(3,6)=(\mathbf{1 5 / 2}) \rho^{\wedge} 2(1-\rho)^{\wedge} 2 \mathrm{f}(1,2)+5 \rho(1-\rho)^{\wedge} 3 \mathrm{f}(2,2) \\
\mathrm{f}(4,6)=(9 / 2) \rho^{\wedge} 3(1-\rho) \mathrm{f}(1,2)+\mathbf{6} \rho^{\wedge} 2(1-\rho)^{\wedge} 2 \mathrm{f}(2,2) \\
\mathrm{f}(4,6)=(30 / 7) \rho^{\wedge} 3(1-\rho) \mathrm{f}(1,2)+(45 / 7) \rho^{\wedge} 2(1-\rho)^{\wedge} 2 \mathrm{f}(2,2) \\
\mathrm{f}(5,6)=\rho^{\wedge} 4 \mathrm{f}(1,2)+4 \rho^{\wedge} 3(1-\rho) \mathrm{f}(2,2) \\
\mathrm{f}(6,6)=\rho^{\wedge} 4 \mathrm{f}(2,2)\end{array}$ \\
\hline & 3 & & & $\begin{array}{l}\mathrm{f}(1,4)=(4 / 3)(1-\rho) \mathrm{f}(1,3) \\
\mathrm{f}(2,4)=\rho \mathrm{f}(1,3)+(1-\rho) \mathrm{f}(2,3) \\
\mathrm{f}(3,4)=\rho \mathrm{f}(2,3)+(1-\rho) \mathrm{f}(3,3) \\
\mathrm{f}(4,4)=\rho \mathrm{f}(3,3)\end{array}$ & $\begin{array}{l}\mathrm{f}(1,5)=(5 / 3)(1-\rho)^{\wedge} 2 \mathrm{f}(1,3) \\
\mathrm{f}(2,5)=(7 / 3) \rho(1-\rho) \mathrm{f}(1,3)+(1-\rho)^{\wedge} 2 \\
\mathrm{f}(2,3) \\
\mathrm{f}(2,5)=(\mathbf{2 0} / \mathbf{9}) \rho(1-\rho) \\
\mathrm{f}(1,3)+(\mathbf{1 0 / 9})(1-\rho)^{\wedge} 2 \mathrm{f}(2,3) \\
\mathrm{f}(3,5)=\rho^{\wedge} 2 \mathrm{f}(1,3)+2 \rho(1-\rho) \\
\mathrm{f}(2,3)+(1-\rho)^{\wedge} 2 \mathrm{f}(3,3) \\
\mathrm{f}(4,5)=\rho^{\wedge} 2 \mathrm{f}(2,3)+2 \rho(1-\rho) \mathrm{f}(3,3) \\
\mathrm{f}(5,5)=\rho^{\wedge} 2 \mathrm{f}(3,3)\end{array}$ & $\begin{array}{l}\mathrm{f}(1,6)=2(1-\rho)^{\wedge} 3 \mathrm{f}(1,3) \\
\mathrm{f}(2,6)=4 \rho(1-\rho)^{\wedge} 2 \mathrm{f}(1,3)+(1-\rho)^{\wedge} 3 \mathrm{f}(2,3) \\
\mathrm{f}(2,6)=(\mathbf{1 5} / 4) \rho(1-\rho)^{\wedge} 2 \mathrm{f}(1,3)+(\mathbf{5} / 4)(1-\rho)^{\wedge} 3 \mathrm{f}(2,3) \\
\mathrm{f}(3,6)=(\mathbf{1 0 / 3}) \rho^{\wedge} 2(1-\rho) \mathrm{f}(1,3)+3 \rho(1-\rho)^{\wedge} 2 \mathrm{f}(2,3)+(1-\rho)^{\wedge} 3 \\
\mathrm{f}(3,3) \\
\mathrm{f}(3,6)=(\mathbf{6 0} / \mathbf{1 9}) \rho^{\wedge} 2(1-\rho) \mathrm{f}(1,3)+(\mathbf{6 0} / \mathbf{1 9}) \rho(1-\rho)^{\wedge} 2 \\
\mathrm{f}(2,3)+(\mathbf{2 0} / \mathbf{1 9})(1-\rho)^{\wedge} 3 \mathrm{f}(3,3) \\
\mathrm{f}(4,6)=\rho^{\wedge} 3 \mathrm{f}(1,3)+3 \rho^{\wedge} 2(1-\rho) \mathrm{f}(2,3)+3 \rho(1-\rho)^{\wedge} 2 \mathrm{f}(3,3) \\
\mathrm{f}(5,6)=\rho^{\wedge} 3 \mathrm{f}(2,3)+3 \rho^{\wedge} 2(1-\rho) \mathrm{f}(3,3) \\
\mathrm{f}(6,6)=\rho^{\wedge} 3 \mathrm{f}(3,3)\end{array}$ \\
\hline
\end{tabular}




\begin{tabular}{|c|c|c|c|c|c|}
\hline \multirow{2}{*}{$\begin{array}{l}\text { Mapping Up } \\
\text { Formulas }\end{array}$} & \multicolumn{5}{|c|}{ Size of System Mapping To (Number of Identical Trains) } \\
\hline & 2 & 3 & 4 & 5 & 6 \\
\hline 4 & & & & $\begin{array}{l}\mathrm{f}(1,5)=(5 / 4)(1-\rho) \mathrm{f}(1,4) \\
\mathrm{f}(2,5)=\rho \mathrm{f}(1,4)+(1-\rho) \mathrm{f}(2,4) \\
\mathrm{f}(3,5)=\rho \mathrm{f}(2,4)+(1-\rho) \mathrm{f}(3,4) \\
\mathrm{f}(4,5)=\rho \mathrm{f}(3,4)+(1-\rho) \mathrm{f}(4,4) \\
\mathrm{f}(5,5)=\rho \mathrm{f}(4,4)\end{array}$ & $\begin{array}{l}\mathrm{f}(1,6)=(3 / 2)(1-\rho)^{\wedge} \mathrm{f}(1,4) \\
\mathrm{f}(2,6)=(9 / 4) \rho(1-\rho) \mathrm{f}(1,4)+(1-\rho)^{\wedge} 2 \mathrm{f}(2,4) \\
\mathrm{f}(2,6)=(15 / 7) \rho(1-\rho) \mathrm{f}(1,4)+(15 / 14)(1-\rho)^{\wedge} 2 \mathrm{f}(2,4) \\
\mathrm{f}(3,6)=\rho^{\wedge} 2 \mathrm{f}(1,4)+2 \rho(1-\rho) \mathrm{f}(2,4)+(1-\rho)^{\wedge} 2 \mathrm{f}(3,4) \\
\mathrm{f}(4,6)=\rho^{\wedge} 2 \mathrm{f}(2,4)+2 \rho(1-\rho) \mathrm{f}(3,4)+(1-\rho)^{\wedge} 2 \mathrm{f}(4,4) \\
\mathrm{f}(5,6)=\rho^{\wedge} 2 \mathrm{f}(3,4)+2 \rho(1-\rho) \mathrm{f}(4,4) \\
\mathrm{f}(6,6)=\rho^{\wedge} 2 \mathrm{f}(4,4)\end{array}$ \\
\hline 5 & & & & & $\begin{array}{l}\mathrm{f}(1,6)=(6 / 5)(1-\rho) \mathrm{f}(1,5) \\
\mathrm{f}(2,6)=\rho \mathrm{f}(1,5)+(1-\rho) \mathrm{f}(2,5) \\
\mathrm{f}(3,6)=\rho \mathrm{f}(2,5)+(1-\rho) \mathrm{f}(3,5) \\
\mathrm{f}(4,6)=\rho \mathrm{f}(3,5)+(1-\rho) \mathrm{f}(4,5) \\
\mathrm{f}(5,6)=\rho \mathrm{f}(4,5)+(1-\rho) \mathrm{f}(5,5) \\
\mathrm{f}(6,6)=\rho \mathrm{f} 5\end{array}$ \\
\hline
\end{tabular}




\section{Can a single prior work for all causes?}

It would be convenient if one prior could be used for every cause type. The causal CCF data presented in this report could be reviewed to determine whether the differences in the various cause groups significantly prohibit the use of a single prior for all causes.

\section{How is the average group size calculated? What is its impact on CCF parameter estimations?}

When using the impact vector and mapping method to estimate CCF parameters, independent events can be mapped from group size $k$ to group size $l$ via the following equation:

$$
n_{I}^{(l)}=\frac{l}{k} n_{I}^{(k)}
$$

However, for the above explicit mapping method, the group size for independent failure events is unavailable in the NRC Reliability and Availability Data System. So, the concept of average group size is introduced and used to map independent events. Assuming $N_{g}$ is the number of groups of size $g$, the average group size can be defined as:

$$
A v g=\sum g N_{g} / \sum N_{g}
$$

where $\Sigma N_{g}$ is the total number of groups and $\Sigma g N_{g}$ is the total number of components. The equivalent number of independent events for group size 1 can be estimated as:

$$
n_{I}^{(l)}=\frac{l}{\sum g N_{g} / \sum N_{g}} n_{I}=\frac{l}{A v g} n_{I}
$$

where $n_{I}$ is the total number of independent component failures.

Without knowing group size information for the associated independent failure events, the current approach as employed in the CCF database software system uses the average group size of the relevant $\mathrm{CCF}$ events to map the independent events. Apparently, the average group size for CCF events is usually different than the average group size that should be used to map independent events.

\section{Will the testing scheme for various components impact priors?}

It is unclear whether different component testing schemes (staggered testing vs. non-staggered or "simultaneous" testing) would impact the prior estimation, or whether separate data analyses are needed for them. One quick thought is that, if (for example) some valves undergo staggered testing and some undergo nearly simultaneous testing, the data for those two kinds of valves must be analyzed separately, since the two kinds of testing will include numerically different alphas. Mixing the two kinds of data would not give a correct result for either valve type. 


\section{FUTURE WORK}

This report documents the current process of developing CCF prior distributions, updating alpha factor priors using data from 1997 through 2015, and developing causal alpha factor priors for five CCF cause groups: Component, Design, Environment, Human, and Other. While these new priors were developed to serve as replacements for the existing ones, the following work were provided in the original study of INL/LTD-17-43723 to address the issues listed in Section 5:

1. Perform sensitivity analysis to understand the impact of different prior distributions on event and condition assessment. NRC has proposed one to identify three or so component groups (one with very sparse data, one with a lot of data, and one somewhere in between), identify three prior distributions (an existing one, a non-informative one, and something in between), calculate alpha parameters for each of the three component groups using three different priors, and plug the resultant alpha factors into one or two SPAR models. This original proposal could be expanded to include the new priors and causal priors developed in the report.

2. Publish the prior development process in national and international conferences/journals and on the NRC website so as to engage outside experts for extensive discussion and improvements.

3. Evaluate the general formula for mapping up CCF data, as described in Item 6 in Section 5. Revise the potential errors in the current mapping up formulas used in the CCF Data Software.

4. Evaluate whether the new approach for estimating the mapping up factor $\rho$ (refer to Item \#4 and \#6 in Section 5) should (and could) be incorporated into the CCF Data Software.

5. Evaluate the calculation of the average group size in the CCF Data Software and its impact on the results (refer to Item \#8 in Section 5). If an alternative determination of the average group size proves more proper, revise the CCF Data Software with the new average group size formula.

6. Evaluate whether the binomial regression treatment of complete CCF events in the prior development process is appropriate.

7. Evaluate whether a single prior could work for all causes.

8. Determine whether different priors should be developed for different component groups for alpha factors and for causal alpha factors. If yes, revise the CCF Data Software accordingly.

9. Evaluate whether there are any other alternative approaches for developing prior distributions, apart from the current impact vector and mapping approach.

Since the original study in 2017 and INL/LTD-17-43723, some of the above suggested works were conducted (e.g., Item \#1 for the impact of prior distributions on CCF parameter estimation), some were planned to be conducted (e.g., \#8 for component-specific priors), while others may be planned in the future based on the inputs from the NRC and other stakeholders. A conference paper [23] was published in August 2018 that describes the CCF prior distribution development process. Another conference paper [24] will be published in November 2021 that presents the more recent CCF research activities that includes the sensitivity analyses to evaluate the impact of various prior distributions might have on $\mathrm{CCF}$ parameter estimations. 


\section{REFERENCES}

[1] Pickard, Lowe, and Garrick, Inc., "Procedures for Treating Common Cause Failures in Safety and Reliability Studies, Volume 1, Procedural Framework and Examples," United States Nuclear Regulatory Commission, NUREG/CR-4780, EPRI NP-5613, January 1988.

[2] Pickard, Lowe, and Garrick, Inc., "Procedures for Treating Common Cause Failures in Safety and Reliability Studies, Volume 2, Analytical Background and Techniques," United States Nuclear Regulatory Commission, NUREG/CR-4780, EPRI NP-5613, January 1989.

[3] J. K. Vaurio, "Common-Cause Failure Models, Data, Quantification," Lappeenranta University of Technology, IEEE Transactions on Reliability, Vol. 48, No. 3, September 1999.

[4] F. Marshall, A. Mosleh and D. Rasmuson, "Common-Cause Failure Database and Analysis System, Volume 1, Overview," United States Nuclear Regulatory Commission, NUREG/CR-6268, INEEL/EXT-97-00696, June 1998.

[5] F. Marshall, A. Mosleh and D. Rasmuson, "Common-Cause Failure Database and Analysis System, Volume 2, Event Definition and Classification," United States Nuclear Regulatory Commission, NUREG/CR-6268, INEEL/EXT-97-00696, June 1998.

[6] F. Marshall, A. Mosleh and D. Rasmuson, "Common-Cause Failure Database and Analysis System, Volume 3, Data Collection and Event Coding," United States Nuclear Regulatory Commission, NUREG/CR-6268, INEEL/EXT-97-00696, June 1998.

[7] K. Kvarfordt, et. al., "Common-Cause Failure Database and Analysis System, Volume 4, CCF Software Reference Manual," United States Nuclear Regulatory Commission, NUREG/CR-6268, INEEL/EXT-97-00696, June 1998.

[8] F. Marshall, D. Rasmuson and A. Mosleh, "Common-Cause Failure Parameter Estimations," United States Nuclear Regulatory Commission, NUREG/CR-5497, INEEL/EXT-97-01328, October 1998.

[9] A. Mosleh, D. Rasmuson and F. Marshall, "Guidelines on Modeling Common-Cause Failures in Probabilistic Risk Assessment," United States Nuclear Regulatory Commission, NUREG/CR-5485, INEEL/EXT-97-01327, November 1998.

[10] T. Wierman, D. Rasmuson and A. Mosleh, "Common-Cause Failure Database and Analysis System: Event Data Collection, Classification, and Coding," United States Nuclear Regulatory Commission, NUREG/CR-6268, Revision 1, September 2007.

[11] U.S. Nuclear Regulatory Commission, "CCF Parameter Estimations, 2003 Update", http://nrcoe.inel.gov/resultsdb/ParamEstSpar/, May 2006.

[12] U.S. Nuclear Regulatory Commission, "CCF Parameter Estimations, 2005 Update", http://nrcoe.inel.gov/resultsdb/ParamEstSpar/, September 2008.

[13] U.S. Nuclear Regulatory Commission, "CCF Parameter Estimations, 2007 Update", http://nrcoe.inel.gov/resultsdb/ParamEstSpar/, September 2008. 
[14] U.S. Nuclear Regulatory Commission, "CCF Parameter Estimations, 2009 Update", http://nrcoe.inel.gov/resultsdb/ParamEstSpar/, January 2011.

[15] U.S. Nuclear Regulatory Commission, "CCF Parameter Estimations, 2010 Update", http://nrcoe.inel.gov/resultsdb/ParamEstSpar/, January 2012.

[16] U.S. Nuclear Regulatory Commission, "CCF Parameter Estimations, 2012 Update", http://nrcoe.inel.gov/resultsdb/ParamEstSpar/, November 2013.

[17] U.S. Nuclear Regulatory Commission, "CCF Parameter Estimations, 2015 Update", http://nrcoe.inel.gov/resultsdb/ParamEstSpar/, October 2016.

[18] U.S. Nuclear Regulatory Commission, "Estimation of Industry-Wide Common-Cause Failure Prior Distributions," 2010.

[19] C. Atwood, "Hits per Trial: Basic Analysis of Binominal Data," EGG-RAAM-11041, 1994.

[20] C. Atwood, et. al., "Handbook of Parameter Estimation for Probabilistic Risk Assessment," United States Nuclear Regulatory Commission, NUREG/CR-6823, SAND2003-3348P, September 2003.

[21] MathWorks, https://www.mathworks.com/products/matlab.html

[22] Z. Ma, et. al., "Feasibility Study of Developing Alternative Common-Cause Failure Model for Event Assessment," Idaho National Laboratory, INL/EXT-21-33376, Revision 1 of INL/LTD-1433376, August 2021.

[23] Z. Ma, J. Schroeder, and C. Smith, "Developing Generic Prior Distributions for Common Cause Failure Alpha Factors," INL/CON-20-57077, Transactions of the American Nuclear Society, Probabilistic Safety Assessment and Management PSAM 14, Los Angeles, California, September 2018.

[24] C. Hunter, et. al., "Research Activities Associated with the Treatment of Potential Common-Cause Failures in Event and Condition Assessment," Accepted by 2021 International Topical Meeting on Probabilistic Safety Assessment and Analysis (PSA 2021), November 2021. 


\section{APPENDIX A}

\section{PRIOR DISTRIBUTIONS IN PREVIOUS CCF PARAMETER ESTIMATION REPORTS}

This Appendix provides a summary of the prior distributions included in NUREG/CR-5485 and the CCF Parameter Estimations Update Reports $\left(2003,2005,2007,2009,2010,2012\right.$, and 2015) ${ }^{\mathrm{c}}$. For simplicity, only alpha factor distributions up to a common cause component group (CCCG) size of 4 are presented for the update reports here. The complete prior distributions for each update report can be located in the corresponding reports via the NRC CCF Results and Databases website:

http://nrcoe.inl.gov/resultsdb/ParamEstSpar/.

The CCF prior distributions - called "No Data (Prior Only)" distributions in the update reports - are published in the 2003 report, and updated in the 2005 report with a data version of 2005/12/31 and in the 2007 report with a data version of 2007/12/31. The CCF prior distributions stopped being updated after 2007. The same CCF prior distributions with the data version of 2007/12/31 are provided in the subsequent update reports (i.e., 2009, 2010, 2012, and 2015).

Since 2007, the update reports provide not only the CCF Prior Distributions, but also "Generic Demand" and "Generic Rate" distributions. While the update reports after 2007 do not update the CCF "No Data (Prior Only)" prior distributions, they do update the "Generic Demand" and "Generic Rate" distributions. (Note that the CCF data used in the 2007 update for the "Generic Demand" and "Generic Rate" distributions starts from 1991, while those used in all of the subsequent update reports start from 1997.)

${ }^{\mathrm{c}}$ NUREG/CR-5497, Common-Cause Failure Parameter Estimations, published in October 1998, does not include CCF prior distributions. It may have used the prior distributions presented in NUREG/CR-5485, Table 5-11 for parameter estimations. 
NUREG/CR-5485, Table 5-11. Generic prior distributions for various system sizes.

\begin{tabular}{|c|c|c|c|c|c|c|c|}
\hline \multirow{2}{*}{$\begin{array}{l}\text { CCCG } \\
\text { Size m }\end{array}$} & \multirow{2}{*}{$\alpha$-Factor } & \multicolumn{2}{|c|}{$\begin{array}{l}\text { Distributions } \\
\text { Parameters }\end{array}$} & \multicolumn{3}{|c|}{ Percentiles } & \multirow{2}{*}{ Mean } \\
\hline & & a & b & $P_{05}$ & $P_{50}$ & $P_{95}$ & \\
\hline 2 & $\begin{array}{l}\alpha_{1} \\
\alpha_{2} \\
\end{array}$ & $\begin{array}{l}9.5300 \\
0.4700\end{array}$ & $\begin{array}{l}0.470 \\
9.530\end{array}$ & $\begin{array}{l}8.20 \mathrm{E}-01 \\
1.42 \mathrm{E}-04\end{array}$ & $\begin{array}{l}9.78 \mathrm{E}-01 \\
2.16 \mathrm{E}-02 \\
\end{array}$ & $\begin{array}{l}1.00 \mathrm{E}-00 \\
1.81 \mathrm{E}-01\end{array}$ & $\begin{array}{l}0.95300 \\
0.04700\end{array}$ \\
\hline 3 & $\begin{array}{l}\alpha_{1} \\
\alpha_{2} \\
\alpha_{3} \\
\end{array}$ & $\begin{array}{r}15.2000 \\
0.3872 \\
0.4128 \\
\end{array}$ & $\begin{array}{r}0.800 \\
15.613 \\
15.587 \\
\end{array}$ & $\begin{array}{l}8.42 \mathrm{E}-01 \\
2.10 \mathrm{E}-05 \\
3.45 \mathrm{E}-05 \\
\end{array}$ & $\begin{array}{l}9.67 \mathrm{E}-01 \\
8.79 \mathrm{E}-03 \\
1.01 \mathrm{E}-02 \\
\end{array}$ & $\begin{array}{l}9.99 \mathrm{E}-01 \\
1.01 \mathrm{E}-01 \\
1.05 \mathrm{E}-01 \\
\end{array}$ & $\begin{array}{l}0.95000 \\
0.02420 \\
0.02580 \\
\end{array}$ \\
\hline 4 & $\begin{array}{l}\alpha_{1} \\
\alpha_{2} \\
\alpha_{3} \\
\alpha_{4}\end{array}$ & $\begin{array}{r}24.7000 \\
0.5538 \\
0.2626 \\
0.4836 \\
\end{array}$ & $\begin{array}{c}1.300 \\
25.446 \\
25.737 \\
25.516 \\
\end{array}$ & $\begin{array}{l}8.67 \mathrm{E}-01 \\
1.44 \mathrm{E}-04 \\
2.98 \mathrm{E}-07 \\
6.29 \mathrm{E}-05 \\
\end{array}$ & $\begin{array}{l}9.61 \mathrm{E}-01 \\
1.08 \mathrm{E}-02 \\
1.99 \mathrm{E}-03 \\
8.42 \mathrm{E}-03\end{array}$ & $\begin{array}{l}9.95 \mathrm{E}-01 \\
7.81 \mathrm{E}-02 \\
4.82 \mathrm{E}-02 \\
7.17 \mathrm{E}-02\end{array}$ & $\begin{array}{l}0.95000 \\
0.02130 \\
0.01010 \\
0.01860\end{array}$ \\
\hline 5 & $\begin{array}{l}\alpha_{1} \\
\alpha_{2} \\
\alpha_{3} \\
\alpha_{4} \\
\alpha_{3} \\
\end{array}$ & $\begin{array}{l}38.042 \\
0.7280 \\
0.4120 \\
0.2336 \\
0.5840 \\
\end{array}$ & $\begin{array}{r}1.958 \\
39.272 \\
39.588 \\
39.766 \\
39.416 \\
\end{array}$ & $\begin{array}{l}8.86 \mathrm{E}-01 \\
3.72 \mathrm{E}-04 \\
1.32 \mathrm{E}-05 \\
4.57 \mathrm{E}-08 \\
1.24 \mathrm{E}-04\end{array}$ & $\begin{array}{l}9.58 \mathrm{E}-01 \\
1.10 \mathrm{E}-02 \\
3.93 \mathrm{E}-03 \\
8.97 \mathrm{E}-04 \\
7.66 \mathrm{E}-03\end{array}$ & $\begin{array}{l}9.91 \mathrm{E}-01 \\
6.05 \mathrm{E}-02 \\
4.22 \mathrm{E}-02 \\
2.89 \mathrm{E}-02 \\
5.27 \mathrm{E}-02 \\
\end{array}$ & $\begin{array}{l}0.95106 \\
0.01820 \\
0.01030 \\
0.00584 \\
0.01460 \\
\end{array}$ \\
\hline 6 & $\begin{array}{l}\alpha_{1} \\
\alpha_{2} \\
\alpha_{3} \\
\alpha_{4} \\
\alpha_{3} \\
\alpha_{6}\end{array}$ & $\begin{array}{r}50.4724 \\
0.7791 \\
0.5406 \\
0.3127 \\
0.2433 \\
0.6519 \\
\end{array}$ & $\begin{array}{r}2.528 \\
52.221 \\
52.459 \\
52.687 \\
52.757 \\
52.348 \\
\end{array}$ & $\begin{array}{l}8.97 \mathrm{E}-01 \\
3.76 \mathrm{E}-04 \\
6.04 \mathrm{E}-05 \\
9.28 \mathrm{E}-07 \\
5.77 \mathrm{E}-08 \\
1.66 \mathrm{E}-04\end{array}$ & $\begin{array}{l}9.58 \mathrm{E}-01 \\
9.20 \mathrm{E}-03 \\
5.02 \mathrm{E}-03 \\
1.56 \mathrm{E}-03 \\
7.67 \mathrm{E}-04 \\
6.93 \mathrm{E}-03\end{array}$ & $\begin{array}{l}9.89 \mathrm{E}-01 \\
4.78 \mathrm{E}-02 \\
3.79 \mathrm{E}-02 \\
2.66 \mathrm{E}-02 \\
2.24 \mathrm{E}-02 \\
4.27 \mathrm{E}-02\end{array}$ & $\begin{array}{l}0.95231 \\
0.01470 \\
0.01020 \\
0.00590 \\
0.00459 \\
0.01230\end{array}$ \\
\hline 7 & $\begin{array}{l}\alpha_{1} \\
\alpha_{2} \\
\alpha_{3} \\
\alpha_{4} \\
\alpha_{3} \\
\alpha_{6} \\
\alpha_{7}\end{array}$ & $\begin{array}{r}74.5360 \\
0.9906 \\
0.6817 \\
0.4891 \\
0.2941 \\
0.2051 \\
0.8034 \\
\end{array}$ & $\begin{array}{r}3.464 \\
77.009 \\
77.318 \\
77.511 \\
77.706 \\
77.795 \\
77.197 \\
\end{array}$ & $\begin{array}{l}9.12 \mathrm{E}-01 \\
6.44 \mathrm{E}-04 \\
1.39 \mathrm{E}-04 \\
2.21 \mathrm{E}-05 \\
3.39 \mathrm{E}-07 \\
3.84 \mathrm{E}-09 \\
2.89 \mathrm{E}-04\end{array}$ & $\begin{array}{l}9.59 \mathrm{E}-01 \\
8.84 \mathrm{E}-03 \\
5.05 \mathrm{E}-03 \\
2.82 \mathrm{E}-03 \\
8.97 \mathrm{E}-04 \\
2.94 \mathrm{E}-04 \\
6.52 \mathrm{E}-03 \\
\end{array}$ & $\begin{array}{l}9.86 \mathrm{E}-01 \\
3.79 \mathrm{E}-02 \\
2.99 \mathrm{E}-02 \\
2.42 \mathrm{E}-02 \\
1.74 \mathrm{E}-02 \\
1.35 \mathrm{E}-02 \\
3.32 \mathrm{E}-02 \\
\end{array}$ & $\begin{array}{l}0.95559 \\
0.01270 \\
0.00874 \\
0.00627 \\
0.00377 \\
0.00263 \\
0.01030 \\
\end{array}$ \\
\hline 8 & $\begin{array}{l}\alpha_{1} \\
\alpha_{2} \\
\alpha_{3} \\
\alpha_{4} \\
\alpha_{3} \\
\alpha_{6} \\
\alpha_{7} \\
\alpha_{8}\end{array}$ & $\begin{array}{r}97.6507 \\
1.1118 \\
0.7915 \\
0.6253 \\
0.4417 \\
0.2581 \\
0.1969 \\
0.9241 \\
\end{array}$ & $\begin{array}{r}4.349 \\
100.888 \\
101.209 \\
101.375 \\
101.558 \\
101.742 \\
101.803 \\
101.076 \\
\end{array}$ & $\begin{array}{l}9.20 \mathrm{E}-01 \\
7.25 \mathrm{E}-04 \\
2.07 \mathrm{E}-04 \\
6.92 \mathrm{E}-05 \\
8.51 \mathrm{E}-06 \\
6.09 \mathrm{E}-08 \\
1.59 \mathrm{E}-09 \\
3.82 \mathrm{E}-04 \\
\end{array}$ & $\begin{array}{l}9.60 \mathrm{E}-01 \\
7.91 \mathrm{E}-03 \\
4.87 \mathrm{E}-03 \\
3.34 \mathrm{E}-03 \\
1.76 \mathrm{E}-03 \\
4.74 \mathrm{E}-04 \\
1.93 \mathrm{E}-04 \\
6.12 \mathrm{E}-03 \\
\end{array}$ & $\begin{array}{l}9.84 \mathrm{E}-01 \\
3.13 \mathrm{E}-02 \\
2.52 \mathrm{E}-02 \\
2.17 \mathrm{E}-02 \\
1.74 \mathrm{E}-02 \\
1.21 \mathrm{E}-02 \\
1.00 \mathrm{E}-02 \\
2.78 \mathrm{E}-02 \\
\end{array}$ & $\begin{array}{l}0.95736 \\
0.01090 \\
0.00776 \\
0.00613 \\
0.00433 \\
0.00253 \\
0.00193 \\
0.00906 \\
\end{array}$ \\
\hline
\end{tabular}


CCF Parameter Estimations 2003, Section 2, No Data (Prior Only)

Section 2.1.1.1, All Failure Modes. No data version is provided.

ALPHA FACTOR DISTRIBUTIONS

\begin{tabular}{|c|c|c|c|c|c|c|c|}
\hline CCCG = 2 & 5 th\% & Mean & Median & 95 th\% & MLE & a & b \\
Alpha Factor & & & & & & & \\
\hline 1 & 0.8783440 & 0.9690420 & 0.9866290 & 0.9999290 & --- & $1.4131 \mathrm{E}+01$ & $4.5144 \mathrm{E}-01$ \\
\hline 2 & $7.24 \mathrm{E}-05$ & $3.09 \mathrm{E}-02$ & $1.33 \mathrm{E}-02$ & $1.21 \mathrm{E}-01$ & $-\cdots$ & $4.5144 \mathrm{E}-01$ & $1.4131 \mathrm{E}+01$ \\
\hline
\end{tabular}

\begin{tabular}{|c|c|c|c|c|c|c|c|}
\hline $\begin{array}{c}\text { CCCG }=3 \\
\text { Alpha Factor }\end{array}$ & 5 th\% & Mean & Median & 95 th\% & MLE & a & b \\
\hline 1 & 0.9148940 & 0.9700020 & 0.9779420 & 0.9979420 & --- & $3.6141 \mathrm{E}+01$ & $1.1176 \mathrm{E}+00$ \\
\hline 2 & $7.72 \mathrm{E}-04$ & $2.28 \mathrm{E}-02$ & $1.50 \mathrm{E}-02$ & $7.15 \mathrm{E}-02$ & $\cdots$ & $8.5035 \mathrm{E}-01$ & $3.6408 \mathrm{E}+01$ \\
\hline 3 & $2.53 \mathrm{E}-07$ & $7.17 \mathrm{E}-03$ & $1.45 \mathrm{E}-03$ & $3.40 \mathrm{E}-02$ & --- & $2.6733 \mathrm{E}-01$ & $3.6991 \mathrm{E}+01$ \\
\hline
\end{tabular}

\begin{tabular}{|c|c|c|c|c|c|c|c|}
\hline $\begin{array}{c}\text { CCCG }=4 \\
\text { Alpha Factor }\end{array}$ & 5 th\% & Mean & Median & 95 th\% & MLE & a & b \\
\hline 1 & 0.9243310 & 0.9682640 & 0.9733830 & 0.9946800 & --- & $5.7379 \mathrm{E}+01$ & $1.8806 \mathrm{E}+00$ \\
\hline 2 & $1.80 \mathrm{E}-03$ & $2.10 \mathrm{E}-02$ & $1.59 \mathrm{E}-02$ & $5.78 \mathrm{E}-02$ & --- & $1.2495 \mathrm{E}+00$ & $5.8010 \mathrm{E}+01$ \\
\hline 3 & $8.65 \mathrm{E}-06$ & $6.92 \mathrm{E}-03$ & $2.61 \mathrm{E}-03$ & $2.84 \mathrm{E}-02$ & $-\cdots$ & $4.1063 \mathrm{E}-01$ & $5.8849 \mathrm{E}+01$ \\
\hline 4 & $1.42 \mathrm{E}-08$ & $3.72 \mathrm{E}-03$ & $4.98 \mathrm{E}-04$ & $1.86 \mathrm{E}-02$ & --- & $2.2054 \mathrm{E}-01$ & $5.9039 \mathrm{E}+01$ \\
\hline
\end{tabular}

\begin{tabular}{|c|c|c|c|c|c|c|c|}
\hline CCCG $=5$ & 5 th\% & Mean & Median & 95 th\% & MLE & a & b \\
Alpha Factor & & & & & & & \\
\hline 1 & 0.9416970 & 0.9705760 & 0.9731470 & 0.9906680 & --- & $1.1687 \mathrm{E}+02$ & $3.5429 \mathrm{E}+00$ \\
\hline 2 & $3.47 \mathrm{E}-03$ & $1.77 \mathrm{E}-02$ & $1.51 \mathrm{E}-02$ & $4.09 \mathrm{E}-02$ & $\cdots-$ & $2.1414 \mathrm{E}+00$ & $1.1827 \mathrm{E}+02$ \\
\hline 3 & $3.67 \mathrm{E}-04$ & $7.95 \mathrm{E}-03$ & $5.44 \mathrm{E}-03$ & $2.40 \mathrm{E}-02$ & --- & $9.5737 \mathrm{E}-01$ & $1.1945 \mathrm{E}+02$ \\
\hline 4 & $1.80 \mathrm{E}-06$ & $3.06 \mathrm{E}-03$ & $1.01 \mathrm{E}-03$ & $1.30 \mathrm{E}-02$ & --- & $3.6878 \mathrm{E}-01$ & $1.2004 \mathrm{E}+02$ \\
\hline 5 & $2.81 \mathrm{E}-20$ & $6.26 \mathrm{E}-04$ & $5.08 \mathrm{E}-07$ & $3.64 \mathrm{E}-03$ & $-\cdots$ & $7.5435 \mathrm{E}-02$ & $1.2033 \mathrm{E}+02$ \\
\hline
\end{tabular}

\begin{tabular}{|c|c|c|c|c|c|c|c|}
\hline $\begin{array}{c}\text { CCCG }=6 \\
\text { Alpha Factor }\end{array}$ & 5 th\% & Mean & Median & 95 th\% & MLE & a & b \\
\hline 1 & 0.9444530 & 0.9705190 & 0.9726530 & 0.9893010 & --- & $1.4121 \mathrm{E}+02$ & $4.2894 \mathrm{E}+00$ \\
\hline 2 & $3.35 \mathrm{E}-03$ & $1.58 \mathrm{E}-02$ & $1.36 \mathrm{E}-02$ & $3.57 \mathrm{E}-02$ & $\cdots-$ & $2.3037 \mathrm{E}+00$ & $1.4319 \mathrm{E}+02$ \\
\hline 3 & $4.86 \mathrm{E}-04$ & $7.54 \mathrm{E}-03$ & $5.44 \mathrm{E}-03$ & $2.17 \mathrm{E}-02$ & $\cdots-$ & $1.0979 \mathrm{E}+00$ & $1.4440 \mathrm{E}+02$ \\
\hline 4 & $3.30 \mathrm{E}-05$ & $3.99 \mathrm{E}-03$ & $2.06 \mathrm{E}-03$ & $1.45 \mathrm{E}-02$ & $\cdots-$ & $5.8178 \mathrm{E}-01$ & $1.4491 \mathrm{E}+02$ \\
\hline 5 & $4.45 \mathrm{E}-09$ & $1.48 \mathrm{E}-03$ & $1.89 \mathrm{E}-04$ & $7.50 \mathrm{E}-03$ & $\cdots-$ & $2.1648 \mathrm{E}-01$ & $1.4528 \mathrm{E}+02$ \\
\hline 6 & $1.23 \mathrm{E}-17$ & $6.15 \mathrm{E}-04$ & $1.80 \mathrm{E}-06$ & $3.59 \mathrm{E}-03$ & $-\cdots$ & $8.9555 \mathrm{E}-02$ & $1.4541 \mathrm{E}+02$ \\
\hline
\end{tabular}

\begin{tabular}{|c|c|c|c|c|c|c|c|}
\hline CCCG $=7$ & 5 th\% & Mean & Median & 95 th\% & MLE & a & b \\
Alpha Factor & & & & & & & \\
\hline 1 & 0.9516920 & 0.9716290 & 0.9729760 & 0.9869580 & --- & $2.2464 \mathrm{E}+02$ & $6.5594 \mathrm{E}+00$ \\
\hline 2 & $4.13 \mathrm{E}-03$ & $1.40 \mathrm{E}-02$ & $1.26 \mathrm{E}-02$ & $2.87 \mathrm{E}-02$ & $\cdots-$ & $3.2518 \mathrm{E}+00$ & $2.2794 \mathrm{E}+02$ \\
\hline 3 & $9.24 \mathrm{E}-04$ & $6.97 \mathrm{E}-03$ & $5.61 \mathrm{E}-03$ & $1.76 \mathrm{E}-02$ & --- & $1.6135 \mathrm{E}+00$ & $2.2958 \mathrm{E}+02$ \\
\hline 4 & $1.95 \mathrm{E}-04$ & $4.16 \mathrm{E}-03$ & $2.85 \mathrm{E}-03$ & $1.26 \mathrm{E}-02$ & --- & $9.6383 \mathrm{E}-01$ & $2.3023 \mathrm{E}+02$ \\
\hline 5 & $1.06 \mathrm{E}-05$ & $2.24 \mathrm{E}-03$ & $1.05 \mathrm{E}-03$ & $8.49 \mathrm{E}-03$ & $-\cdots$ & $5.1822 \mathrm{E}-01$ & $2.3068 \mathrm{E}+02$ \\
\hline 6 & $2.00 \mathrm{E}-10$ & $7.87 \mathrm{E}-04$ & $6.28 \mathrm{E}-05$ & $4.15 \mathrm{E}-03$ & --- & $1.8209 \mathrm{E}-01$ & $2.3101 \mathrm{E}+02$ \\
\hline 7 & $1.08 \mathrm{E}-46$ & $1.29 \mathrm{E}-04$ & $2.31 \mathrm{E}-13$ & $5.03 \mathrm{E}-04$ & --- & $3.0005 \mathrm{E}-02$ & $2.3116 \mathrm{E}+02$ \\
\hline
\end{tabular}

\begin{tabular}{|c|c|c|c|c|c|c|c|}
\hline CCCG $=8$ & 5 th\% & Mean & Median & 95 th\% & MLE & a & b \\
Alpha Factor & & & & & & & \\
\hline 1 & 0.9540630 & 0.9722080 & 0.9733600 & 0.9864020 & --- & $2.6325 \mathrm{E}+02$ & $7.5254 \mathrm{E}+00$ \\
\hline 2 & $4.02 \mathrm{E}-03$ & $1.29 \mathrm{E}-02$ & $1.17 \mathrm{E}-02$ & $2.58 \mathrm{E}-02$ & --- & $3.4977 \mathrm{E}+00$ & $2.6727 \mathrm{E}+02$ \\
\hline 3 & $9.44 \mathrm{E}-04$ & $6.40 \mathrm{E}-03$ & $5.23 \mathrm{E}-03$ & $1.58 \mathrm{E}-02$ & --- & $1.7348 \mathrm{E}+00$ & $2.6904 \mathrm{E}+02$ \\
\hline 4 & $2.55 \mathrm{E}-04$ & $4.02 \mathrm{E}-03$ & $2.89 \mathrm{E}-03$ & $1.16 \mathrm{E}-02$ & $-\cdots$ & $1.0910 \mathrm{E}+00$ & $2.6968 \mathrm{E}+02$ \\
\hline 5 & $4.14 \mathrm{E}-05$ & $2.53 \mathrm{E}-03$ & $1.46 \mathrm{E}-03$ & $8.68 \mathrm{E}-03$ & $\cdots-$ & $6.8763 \mathrm{E}-01$ & $2.7008 \mathrm{E}+02$ \\
\hline 6 & $6.49 \mathrm{E}-07$ & $1.32 \mathrm{E}-03$ & $4.24 \mathrm{E}-04$ & $5.72 \mathrm{E}-03$ & --- & $3.5982 \mathrm{E}-01$ & $2.7041 \mathrm{E}+02$ \\
\hline 7 & $1.78 \mathrm{E}-14$ & $4.32 \mathrm{E}-04$ & $6.14 \mathrm{E}-06$ & $2.47 \mathrm{E}-03$ & --- & $1.1715 \mathrm{E}-01$ & $2.7065 \mathrm{E}+02$ \\
\hline 8 & $3.39 \mathrm{E}-38$ & $1.38 \mathrm{E}-04$ & $1.90 \mathrm{E}-11$ & $6.36 \mathrm{E}-04$ & --- & $3.7386 \mathrm{E}-02$ & $2.7073 \mathrm{E}+02$ \\
\hline
\end{tabular}


CCF Parameter Estimations 2005, Section 3, No Data (Prior Only)

Section 3, No Data (Prior Only). Data Version 2005/12/31

ALPHA FACTOR DISTRIBUTIONS

\begin{tabular}{|c|c|c|c|c|c|c|c|}
\hline \multicolumn{4}{|c|}{ Data Version: } & \multicolumn{4}{|c|}{$2005 / 12 / 31$} \\
\hline $\begin{array}{c}C C C G=2 \\
\text { Alpha Factor }\end{array}$ & 5th\% & Mean & Median & 95 th $\%$ & MLE & $\mathrm{a}$ & b \\
\hline 1 & 0.8386830 & 0.9593170 & 0.9828300 & 0.9999250 & $\ldots$ & $1.0246 \mathrm{E}+01$ & $4.3452 \mathrm{E}-01$ \\
\hline 2 & $7.69 \mathrm{E}-05$ & $4.06 \mathrm{E}-02$ & $1.71 \mathrm{E}-02$ & $1.61 \mathrm{E}-01$ & $\ldots$ & $4.3452 \mathrm{E}-01$ & $1.0246 \mathrm{E}+01$ \\
\hline
\end{tabular}

\begin{tabular}{|c|c|c|c|c|c|c|c|}
\hline $\begin{array}{c}C C C G=3 \\
\text { Alpha Factor }\end{array}$ & 5th\% & Mean & Median & 95th\% & MLE & a & b \\
\hline 1 & 0.8979020 & 0.9640890 & 0.9736330 & 0.9976040 & $-\ldots$ & $2.9555 \mathrm{E}+01$ & $1.1008 \mathrm{E}+00$ \\
\hline 2 & $8.71 \mathrm{E}-04$ & $2.71 \mathrm{E}-02$ & $1.77 \mathrm{E}-02$ & $8.57 \mathrm{E}-02$ & -- & $8.3366 \mathrm{E}-01$ & $2.9822 \mathrm{E}+01$ \\
\hline 3 & $3.07 \mathrm{E}-07$ & $8.71 \mathrm{E}-03$ & $1.77 \mathrm{E}-03$ & $4.13 \mathrm{E}-02$ & --- & $2.6722 \mathrm{E}-01$ & $3.0388 \mathrm{E}+01$ \\
\hline
\end{tabular}

\begin{tabular}{|c|c|c|c|c|c|c|c|}
\hline $\begin{array}{c}\text { CCCG }=4 \\
\text { Alpha Factor }\end{array}$ & $\mathbf{5 t h} \%$ & Mean & Median & $\mathbf{9 5 t h} \%$ & MLE & $\mathbf{a}$ & b \\
\hline 1 & 0.9077330 & 0.9613450 & 0.9675780 & 0.9936050 & -- & $4.6136 \mathrm{E}+01$ & $1.8550 \mathrm{E}+00$ \\
\hline 2 & $2.13 \mathrm{E}-03$ & $2.55 \mathrm{E}-02$ & $1.93 \mathrm{E}-02$ & $7.04 \mathrm{E}-02$ & --- & $1.2281 \mathrm{E}+00$ & $4.6763 \mathrm{E}+01$ \\
\hline 3 & $9.52 \mathrm{E}-06$ & $8.42 \mathrm{E}-03$ & $3.13 \mathrm{E}-03$ & $3.47 \mathrm{E}-02$ &.-- & $4.0431 \mathrm{E}-01$ & $4.7586 \mathrm{E}+01$ \\
\hline 4 & $2.00 \mathrm{E}-08$ & $4.64 \mathrm{E}-03$ & $6.37 \mathrm{E}-04$ & $2.32 \mathrm{E}-02$ & -- & $2.2267 \mathrm{E}-01$ & $4.7768 \mathrm{E}+01$ \\
\hline
\end{tabular}

\begin{tabular}{|c|c|c|c|c|c|c|c|}
\hline $\begin{array}{c}\text { CCCG }=5 \\
\text { Alpha Factor }\end{array}$ & $\mathbf{5 t h} \%$ & Mean & Median & $\mathbf{9 5 t h} \%$ & MLE & a & b \\
\hline 1 & 0.9298110 & 0.9645110 & 0.9675740 & 0.9887220 & -- & $9.6161 \mathrm{E}+01$ & $3.5382 \mathrm{E}+00$ \\
\hline 2 & $4.08 \mathrm{E}-03$ & $2.12 \mathrm{E}-02$ & $1.80 \mathrm{E}-02$ & $4.89 \mathrm{E}-02$ & -- & $2.1142 \mathrm{E}+00$ & $9.7585 \mathrm{E}+01$ \\
\hline 3 & $4.79 \mathrm{E}-04$ & $9.80 \mathrm{E}-03$ & $6.77 \mathrm{E}-03$ & $2.94 \mathrm{E}-02$ & -- & $9.7738 \mathrm{E}-01$ & $9.8721 \mathrm{E}+01$ \\
\hline 4 & $2.47 \mathrm{E}-06$ & $3.75 \mathrm{E}-03$ & $1.26 \mathrm{E}-03$ & $1.59 \mathrm{E}-02$ & -- & $3.7439 \mathrm{E}-01$ & $9.9324 \mathrm{E}+01$ \\
\hline 5 & $5.98 \mathrm{E}-21$ & $7.25 \mathrm{E}-04$ & $4.10 \mathrm{E}-07$ & $4.19 \mathrm{E}-03$ & -- & $7.2277 \mathrm{E}-02$ & $9.9627 \mathrm{E}+01$ \\
\hline
\end{tabular}

\begin{tabular}{|c|c|c|c|c|c|c|c|}
\hline $\begin{array}{c}C C C G=6 \\
\text { Alpha Factor }\end{array}$ & 5th\% & Mean & Median & 95 th $\%$ & MLE & a & b \\
\hline 1 & 0.9335790 & 0.9647030 & 0.9672350 & 0.9871780 & $\ldots$ & $1.1694 \mathrm{E}+02$ & $4.2786 \mathrm{E}+00$ \\
\hline 2 & $3.80 \mathrm{E}-03$ & $1.84 \mathrm{E}-02$ & $1.58 \mathrm{E}-02$ & $4.19 \mathrm{E}-02$ & $\ldots$ & $2.2392 \mathrm{E}+00$ & $1.1897 \mathrm{E}+02$ \\
\hline 3 & $6.63 \mathrm{E}-04$ & $9.41 \mathrm{E}-03$ & $6.89 \mathrm{E}-03$ & $2.68 \mathrm{E}-02$ & $\ldots$ & $1.1418 \mathrm{E}+00$ & $1.2007 \mathrm{E}+02$ \\
\hline 4 & $4.37 \mathrm{E}-05$ & $4.88 \mathrm{E}-03$ & $2.56 \mathrm{E}-03$ & $1.76 \mathrm{E}-02$ & $\ldots$ & $5.9222 \mathrm{E}-01$ & $1.2062 \mathrm{E}+02$ \\
\hline 5 & $7.66 \mathrm{E}-09$ & $1.83 \mathrm{E}-03$ & $2.48 \mathrm{E}-04$ & $9.18 \mathrm{E}-03$ & $\ldots$ & $2.2220 \mathrm{E}-01$ & $1.2099 \mathrm{E}+02$ \\
\hline 6 & $1.16 \mathrm{E}-18$ & $6.86 \mathrm{E}-04$ & $1.20 \mathrm{E}-06$ & $4.00 \mathrm{E}-03$ & $\ldots$ & $8.3237 \mathrm{E}-02$ & $1.2113 \mathrm{E}+02$ \\
\hline
\end{tabular}

\begin{tabular}{|c|c|c|c|c|c|c|c|}
\hline $\begin{array}{c}\text { CCCG }=7 \\
\text { Alpha Factor }\end{array}$ & 5th\% & Mean & Median & $\mathbf{9 5 t h} \%$ & MLE & a & b \\
\hline 1 & 0.9422570 & 0.9661670 & 0.9677850 & 0.9845420 & $-\cdots$ & $1.8405 \mathrm{E}+02$ & $6.4449 \mathrm{E}+00$ \\
\hline 2 & $4.56 \mathrm{E}-03$ & $1.62 \mathrm{E}-02$ & $1.45 \mathrm{E}-02$ & $3.35 \mathrm{E}-02$ & -- & $3.0878 \mathrm{E}+00$ & $1.8740 \mathrm{E}+02$ \\
\hline 3 & $1.15 \mathrm{E}-03$ & $8.56 \mathrm{E}-03$ & $6.91 \mathrm{E}-03$ & $2.16 \mathrm{E}-02$ & -- & $1.6312 \mathrm{E}+00$ & $1.8886 \mathrm{E}+02$ \\
\hline 4 & $2.60 \mathrm{E}-04$ & $5.19 \mathrm{E}-03$ & $3.59 \mathrm{E}-03$ & $1.55 \mathrm{E}-02$ & $-\cdots$ & $9.8887 \mathrm{E}-01$ & $1.8950 \mathrm{E}+02$ \\
\hline 5 & $1.34 \mathrm{E}-05$ & $2.73 \mathrm{E}-03$ & $1.29 \mathrm{E}-03$ & $1.03 \mathrm{E}-02$ & -- & $5.2177 \mathrm{E}-01$ & $1.8997 \mathrm{E}+02$ \\
\hline 6 & $3.52 \mathrm{E}-10$ & $9.77 \mathrm{E}-04$ & $8.35 \mathrm{E}-05$ & $5.13 \mathrm{E}-03$ & -- & $1.8628 \mathrm{E}-01$ & $1.9030 \mathrm{E}+02$ \\
\hline 7 & $5.33 \mathrm{E}-48$ & $1.52 \mathrm{E}-04$ & $1.33 \mathrm{E}-13$ & $5.74 \mathrm{E}-04$ & --- & $2.9071 \mathrm{E}-02$ & $1.9046 \mathrm{E}+02$ \\
\hline
\end{tabular}

\begin{tabular}{|c|c|c|c|c|c|c|c|}
\hline $\begin{array}{c}C C C G=8 \\
\text { Alpha Factor }\end{array}$ & 5th\% & Mean & Median & 95 th $\%$ & MLE & a & b \\
\hline 1 & 0.9454620 & 0.9670150 & 0.9683850 & 0.9838980 & $\ldots$ & $2.1873 \mathrm{E}+02$ & $7.4609 \mathrm{E}+00$ \\
\hline 2 & $4.45 \mathrm{E}-03$ & $1.47 \mathrm{E}-02$ & $1.33 \mathrm{E}-02$ & $2.99 \mathrm{E}-02$ & $\ldots$ & $3.3447 \mathrm{E}+00$ & $2.2284 \mathrm{E}+02$ \\
\hline 3 & $1.13 \mathrm{E}-03$ & $7.68 \mathrm{E}-03$ & $6.29 \mathrm{E}-03$ & $1.90 \mathrm{E}-02$ & $\ldots$ & $1.7384 \mathrm{E}+00$ & $2.2445 \mathrm{E}+02$ \\
\hline 4 & $3.58 \mathrm{E}-04$ & $5.06 \mathrm{E}-03$ & $3.70 \mathrm{E}-03$ & $1.44 \mathrm{E}-02$ & $\ldots$ & $1.1465 \mathrm{E}+00$ & $2.2504 \mathrm{E}+02$ \\
\hline 5 & $5.70 \mathrm{E}-05$ & $3.13 \mathrm{E}-03$ & $1.84 \mathrm{E}-03$ & $1.06 \mathrm{E}-02$ & $\ldots$ & $7.0833 \mathrm{E}-01$ & $2.2548 \mathrm{E}+02$ \\
\hline 6 & $9.18 \mathrm{E}-07$ & $1.62 \mathrm{E}-03$ & $5.31 \mathrm{E}-04$ & $6.94 \mathrm{E}-03$ & $\ldots$ & $3.6696 \mathrm{E}-01$ & $2.2582 \mathrm{E}+02$ \\
\hline 7 & $7.20 \mathrm{E}-14$ & $5.43 \mathrm{E}-04$ & $9.77 \mathrm{E}-06$ & $3.09 \mathrm{E}-03$ & $\ldots$ & $1.2297 \mathrm{E}-01$ & $2.2606 \mathrm{E}+02$ \\
\hline 8 & $1.34 \mathrm{E}-42$ & $1.46 \mathrm{E}-04$ & $2.08 \mathrm{E}-12$ & $6.19 \mathrm{E}-04$ & $\ldots$ & $3.3124 \mathrm{E}-02$ & $2.2615 \mathrm{E}+02$ \\
\hline
\end{tabular}


CCF Parameter Estimations 2007, Section 3, No Data (Prior Only)

\section{Section 3.1.3, CCF Prior Distribution, Data Version 2007/12/31}

\begin{tabular}{|c|c|}
\hline Data Version: & $2007 / 12 / 31$ \\
\hline
\end{tabular}

Total Number of Independent Failure Events: 0

Total Number of Common-Cause Failure Events: 0

ALPHA FACTOR DISTRIBUTIONS

$\mathrm{CCCG}=2$

\begin{tabular}{|c|c|c|c|c|c|c|c|}
\hline Alpha Factor & 5 th $\%$ & Mean & Median & 95 th $\%$ & MLE & a & b \\
\hline 1 & 0.8993200 & 0.9742690 & 0.9887700 & 0.9999290 & --- & $1.7418 \mathrm{E}+01$ & $4.6002 \mathrm{E}-01$ \\
\hline 2 & $6.65 \mathrm{E}-05$ & $2.57 \mathrm{E}-02$ & $1.12 \mathrm{E}-02$ & $1.00 \mathrm{E}-01$ & --- & $4.6002 \mathrm{E}-01$ & $1.7418 \mathrm{E}+01$ \\
\hline
\end{tabular}

$\mathrm{CCCG}=3$

\begin{tabular}{|c|c|c|c|c|c|c|c|}
\hline Alpha Factor & 5 th\% & Mean & Median & 95 th\% & MLE & a & b \\
\hline 1 & 0.9306240 & 0.9755060 & 0.9819700 & 0.9982830 & ---- & $4.5105 \mathrm{E}+01$ & $1.1325 \mathrm{E}+00$ \\
\hline 2 & $6.61 \mathrm{E}-04$ & $1.87 \mathrm{E}-02$ & $1.23 \mathrm{E}-02$ & $5.84 \mathrm{E}-02$ & --- & $8.6476 \mathrm{E}-01$ & $4.5372 \mathrm{E}+01$ \\
\hline 3 & $2.07 \mathrm{E}-07$ & $5.79 \mathrm{E}-03$ & $1.17 \mathrm{E}-03$ & $2.74 \mathrm{E}-02$ & --- & $2.6776 \mathrm{E}-01$ & $4.5969 \mathrm{E}+01$ \\
\hline
\end{tabular}

$\mathrm{CCCG}=4$

\begin{tabular}{|c|c|c|c|c|c|c|c|}
\hline Alpha Factor & 5 th $\%$ & Mean & Median & 95 th\% & MLE & a & b \\
\hline 1 & 0.9380870 & 0.9740820 & 0.9782970 & 0.9956540 & ---- & $7.0868 \mathrm{E}+01$ & $1.8856 \mathrm{E}+00$ \\
\hline 2 & $1.43 \mathrm{E}-03$ & $1.70 \mathrm{E}-02$ & $1.28 \mathrm{E}-02$ & $4.69 \mathrm{E}-02$ & --- & $1.2400 \mathrm{E}+00$ & $7.1513 \mathrm{E}+01$ \\
\hline 3 & $9.66 \mathrm{E}-06$ & $5.89 \mathrm{E}-03$ & $2.32 \mathrm{E}-03$ & $2.38 \mathrm{E}-02$ & --- & $4.2870 \mathrm{E}-01$ & $7.2324 \mathrm{E}+01$ \\
\hline 4 & $9.21 \mathrm{E}-09$ & $2.98 \mathrm{E}-03$ & $3.83 \mathrm{E}-04$ & $1.50 \mathrm{E}-02$ & --- & $2.1695 \mathrm{E}-01$ & $7.2536 \mathrm{E}+01$ \\
\hline
\end{tabular}

$\mathrm{CCCG}=5$

\begin{tabular}{|c|c|c|c|c|c|c|c|}
\hline Alpha Factor & 5 th\% & Mean & Median & 95 th\% & MLE & a & b \\
\hline 1 & 0.9521790 & 0.9760740 & 0.9782400 & 0.9925770 & ---- & $1.4106 \mathrm{E}+02$ & $3.4576 \mathrm{E}+00$ \\
\hline 2 & $2.59 \mathrm{E}-03$ & $1.41 \mathrm{E}-02$ & $1.19 \mathrm{E}-02$ & $3.30 \mathrm{E}-02$ & --- & $2.0400 \mathrm{E}+00$ & $1.4247 \mathrm{E}+02$ \\
\hline 3 & $3.01 \mathrm{E}-04$ & $6.59 \mathrm{E}-03$ & $4.50 \mathrm{E}-03$ & $2.00 \mathrm{E}-02$ & ---- & $9.5369 \mathrm{E}-01$ & $1.4356 \mathrm{E}+02$ \\
\hline 4 & $2.21 \mathrm{E}-06$ & $2.67 \mathrm{E}-03$ & $9.37 \mathrm{E}-04$ & $1.12 \mathrm{E}-02$ & --- & $3.8684 \mathrm{E}-01$ & $1.4413 \mathrm{E}+02$ \\
\hline 5 & $5.61 \mathrm{E}-20$ & $5.33 \mathrm{E}-04$ & $5.18 \mathrm{E}-07$ & $3.10 \mathrm{E}-03$ & --- & $7.7129 \mathrm{E}-02$ & $1.4444 \mathrm{E}+02$ \\
\hline
\end{tabular}

$\mathrm{CCCG}=6$

\begin{tabular}{|c|c|c|c|c|c|c|c|}
\hline Alpha Factor & 5 th\% & Mean & Median & 95 th\% & MLE & a & b \\
\hline 1 & 0.9553700 & 0.9762820 & 0.9779970 & 0.9913440 & ---- & $1.7893 \mathrm{E}+02$ & $4.3470 \mathrm{E}+00$ \\
\hline 2 & $2.60 \mathrm{E}-03$ & $1.24 \mathrm{E}-02$ & $1.07 \mathrm{E}-02$ & $2.81 \mathrm{E}-02$ & --- & $2.2804 \mathrm{E}+00$ & $1.8099 \mathrm{E}+02$ \\
\hline 3 & $4.16 \mathrm{E}-04$ & $6.13 \mathrm{E}-03$ & $4.45 \mathrm{E}-03$ & $1.75 \mathrm{E}-02$ & --- & $1.1245 \mathrm{E}+00$ & $1.8215 \mathrm{E}+02$ \\
\hline 4 & $3.82 \mathrm{E}-05$ & $3.40 \mathrm{E}-03$ & $1.85 \mathrm{E}-03$ & $1.20 \mathrm{E}-02$ & --- & $6.2471 \mathrm{E}-01$ & $1.8265 \mathrm{E}+02$ \\
\hline 5 & $1.60 \mathrm{E}-08$ & $1.32 \mathrm{E}-03$ & $2.18 \mathrm{E}-04$ & $6.46 \mathrm{E}-03$ & --- & $2.4272 \mathrm{E}-01$ & $1.8303 \mathrm{E}+02$ \\
\hline 6 & $1.26 \mathrm{E}-20$ & $4.07 \mathrm{E}-04$ & $3.05 \mathrm{E}-07$ & $2.36 \mathrm{E}-03$ & --- & $7.4722 \mathrm{E}-02$ & $1.8320 \mathrm{E}+02$ \\
\hline
\end{tabular}

$\mathrm{CCCG}=7$

\begin{tabular}{|c|c|c|c|c|c|c|c|}
\hline Alpha Factor & 5 th\% & Mean & Median & 95 th\% & MLE & a & b \\
\hline 1 & 0.9603690 & 0.9769760 & 0.9781320 & 0.9896440 & ---- & $2.6720 \mathrm{E}+02$ & $6.2971 \mathrm{E}+00$ \\
\hline 2 & $3.14 \mathrm{E}-03$ & $1.12 \mathrm{E}-02$ & $1.00 \mathrm{E}-02$ & $2.33 \mathrm{E}-02$ & --- & $3.0721 \mathrm{E}+00$ & $2.7042 \mathrm{E}+02$ \\
\hline 3 & $6.66 \mathrm{E}-04$ & $5.55 \mathrm{E}-03$ & $4.40 \mathrm{E}-03$ & $1.43 \mathrm{E}-02$ & --- & $1.5182 \mathrm{E}+00$ & $2.7197 \mathrm{E}+02$ \\
\hline 4 & $1.58 \mathrm{E}-04$ & $3.48 \mathrm{E}-03$ & $2.37 \mathrm{E}-03$ & $1.06 \mathrm{E}-02$ & --- & $9.5310 \mathrm{E}-01$ & $2.7254 \mathrm{E}+02$ \\
\hline 5 & $1.00 \mathrm{E}-05$ & $1.93 \mathrm{E}-03$ & $9.22 \mathrm{E}-04$ & $7.26 \mathrm{E}-03$ & --- & $5.2795 \mathrm{E}-01$ & $2.7296 \mathrm{E}+02$ \\
\hline 6 & $4.58 \mathrm{E}-10$ & $7.08 \mathrm{E}-04$ & $6.75 \mathrm{E}-05$ & $3.68 \mathrm{E}-03$ & ---- & $1.9373 \mathrm{E}-01$ & $2.7330 \mathrm{E}+02$ \\
\hline 7 & $5.03 \mathrm{E}-44$ & $1.17 \mathrm{E}-04$ & $8.41 \mathrm{E}-13$ & $4.81 \mathrm{E}-04$ & --- & $3.2027 \mathrm{E}-02$ & $2.7346 \mathrm{E}+02$ \\
\hline
\end{tabular}

$\mathrm{CCCG}=8$

\begin{tabular}{|c|c|c|c|c|c|c|c|}
\hline Alpha Factor & 5 th\% & Mean & Median & 95 th\% & MLE & a & b \\
\hline 1 & 0.9622170 & 0.9773660 & 0.9783580 & 0.9891370 & ---- & $3.1221 \mathrm{E}+02$ & $7.2302 \mathrm{E}+00$ \\
\hline 2 & $3.13 \mathrm{E}-03$ & $1.04 \mathrm{E}-02$ & $9.45 \mathrm{E}-03$ & $2.12 \mathrm{E}-02$ & --- & $3.3414 \mathrm{E}+00$ & $3.1609 \mathrm{E}+02$ \\
\hline 3 & $6.67 \mathrm{E}-04$ & $5.04 \mathrm{E}-03$ & $4.06 \mathrm{E}-03$ & $1.28 \mathrm{E}-02$ & --- & $1.6130 \mathrm{E}+00$ & $3.1782 \mathrm{E}+02$ \\
\hline 4 & $1.86 \mathrm{E}-04$ & $3.26 \mathrm{E}-03$ & $2.30 \mathrm{E}-03$ & $9.62 \mathrm{E}-03$ & ---- & $1.0438 \mathrm{E}+00$ & $3.1839 \mathrm{E}+02$ \\
\hline 5 & $3.88 \mathrm{E}-05$ & $2.20 \mathrm{E}-03$ & $1.28 \mathrm{E}-03$ & $7.47 \mathrm{E}-03$ & --- & $7.0280 \mathrm{E}-01$ & $3.1873 \mathrm{E}+02$ \\
\hline 6 & $5.77 \mathrm{E}-07$ & $1.13 \mathrm{E}-03$ & $3.63 \mathrm{E}-04$ & $4.86 \mathrm{E}-03$ & --- & $3.6184 \mathrm{E}-01$ & $3.1907 \mathrm{E}+02$ \\
\hline 7 & $1.19 \mathrm{E}-13$ & $3.98 \mathrm{E}-04$ & $8.44 \mathrm{E}-06$ & $2.25 \mathrm{E}-03$ & ---- & $1.2739 \mathrm{E}-01$ & $3.1931 \mathrm{E}+02$ \\
\hline 8 & $5.47 \mathrm{E}-36$ & $1.25 \mathrm{E}-04$ & $5.43 \mathrm{E}-11$ & $6.01 \mathrm{E}-04$ & ---- & $4.0005 \mathrm{E}-02$ & $3.1940 \mathrm{E}+02$ \\
\hline
\end{tabular}


CCF Parameter Estimations 2007, Section 3

Section 3.1.1, Generic Demand CCF Prior Distribution: CCF-DEM

\begin{tabular}{|c|c|}
\hline Failure Mode : & FAIL TO CLOSE (NORMALLY OPEN) \\
& FAIL TO OPEN (NORMALLY CLOSED) \\
& FAIL TO START \\
& FAIL TO STOP \\
\hline Start Date: & $1991 / 01 / 01$ \\
\hline Data Version : & $2007 / 12 / 31$ \\
\hline
\end{tabular}

Total Number of Independent Failure Events: 5722

Total Number of Common-Cause Failure Events: 375

\section{ALPHA FACTOR DISTRIBUTIONS}

$\mathrm{CCCG}=2$

\begin{tabular}{|c|c|c|c|c|c|c|c|}
\hline Alpha Factor & 5 th $\%$ & Mean & Median & 95 th $\%$ & MLE & a & b \\
\hline 1 & 0.9575610 & 0.9648240 & 0.9649860 & 0.9715280 & 0.9647330 & $1.8100 \mathrm{E}+03$ & $6.5990 \mathrm{E}+01$ \\
\hline 2 & $2.85 \mathrm{E}-02$ & $3.52 \mathrm{E}-02$ & $3.50 \mathrm{E}-02$ & $4.24 \mathrm{E}-02$ & $3.53 \mathrm{E}-02$ & $6.5990 \mathrm{E}+01$ & $1.8100 \mathrm{E}+03$ \\
\hline
\end{tabular}

$\mathrm{CCCG}=3$

\begin{tabular}{|c|c|c|c|c|c|c|c|}
\hline Alpha Factor & 5 th $\%$ & Mean & Median & 95 th $\%$ & MLE & a & b \\
\hline 1 & 0.9579120 & 0.9639110 & 0.9640230 & 0.9695270 & 0.9637140 & $2.6833 \mathrm{E}+03$ & $1.0046 \mathrm{E}+02$ \\
\hline 2 & $1.94 \mathrm{E}-02$ & $2.39 \mathrm{E}-02$ & $2.38 \mathrm{E}-02$ & $2.89 \mathrm{E}-02$ & $2.40 \mathrm{E}-02$ & $6.6566 \mathrm{E}+01$ & $2.7172 \mathrm{E}+03$ \\
\hline 3 & $8.97 \mathrm{E}-03$ & $1.22 \mathrm{E}-02$ & $1.21 \mathrm{E}-02$ & $1.58 \mathrm{E}-02$ & $1.23 \mathrm{E}-02$ & $3.3898 \mathrm{E}+01$ & $2.7499 \mathrm{E}+03$ \\
\hline
\end{tabular}

$\mathrm{CCCG}=4$

\begin{tabular}{|c|c|c|c|c|c|c|c|}
\hline Alpha Factor & 5 th $\%$ & Mean & Median & 95 th\% & MLE & a & b \\
\hline 1 & 0.9596480 & 0.9647820 & 0.9648640 & 0.9696300 & 0.9645940 & $3.5506 \mathrm{E}+03$ & $1.2961 \mathrm{E}+02$ \\
\hline 2 & $1.59 \mathrm{E}-02$ & $1.95 \mathrm{E}-02$ & $1.94 \mathrm{E}-02$ & $2.34 \mathrm{E}-02$ & $1.96 \mathrm{E}-02$ & $7.1769 \mathrm{E}+01$ & $3.6084 \mathrm{E}+03$ \\
\hline 3 & $7.69 \mathrm{E}-03$ & $1.03 \mathrm{E}-02$ & $1.02 \mathrm{E}-02$ & $1.31 \mathrm{E}-02$ & $1.04 \mathrm{E}-02$ & $3.7766 \mathrm{E}+01$ & $3.6424 \mathrm{E}+03$ \\
\hline 4 & $3.62 \mathrm{E}-03$ & $5.45 \mathrm{E}-03$ & $5.37 \mathrm{E}-03$ & $7.59 \mathrm{E}-03$ & $5.50 \mathrm{E}-03$ & $2.0074 \mathrm{E}+01$ & $3.6601 \mathrm{E}+03$ \\
\hline
\end{tabular}

$\mathrm{CCCG}=5$

\begin{tabular}{|c|c|c|c|c|c|c|c|}
\hline Alpha Factor & 5 th\% & Mean & Median & 95 th $\%$ & MLE & a & b \\
\hline 1 & 0.9634500 & 0.9678320 & 0.9679010 & 0.9719870 & 0.9675650 & $4.4690 \mathrm{E}+03$ & $1.4854 \mathrm{E}+02$ \\
\hline 2 & $1.20 \mathrm{E}-02$ & $1.48 \mathrm{E}-02$ & $1.47 \mathrm{E}-02$ & $1.78 \mathrm{E}-02$ & $1.48 \mathrm{E}-02$ & $6.8136 \mathrm{E}+01$ & $4.5494 \mathrm{E}+03$ \\
\hline 3 & $6.70 \mathrm{E}-03$ & $8.84 \mathrm{E}-03$ & $8.77 \mathrm{E}-03$ & $1.12 \mathrm{E}-02$ & $8.91 \mathrm{E}-03$ & $4.0807 \mathrm{E}+01$ & $4.5767 \mathrm{E}+03$ \\
\hline 4 & $4.33 \mathrm{E}-03$ & $6.08 \mathrm{E}-03$ & $6.01 \mathrm{E}-03$ & $8.08 \mathrm{E}-03$ & $6.19 \mathrm{E}-03$ & $2.8077 \mathrm{E}+01$ & $4.5895 \mathrm{E}+03$ \\
\hline 5 & $1.42 \mathrm{E}-03$ & $2.49 \mathrm{E}-03$ & $2.42 \mathrm{E}-03$ & $3.81 \mathrm{E}-03$ & $2.56 \mathrm{E}-03$ & $1.1519 \mathrm{E}+01$ & $4.6060 \mathrm{E}+03$ \\
\hline
\end{tabular}

$\mathrm{CCCG}=6$

\begin{tabular}{|c|c|c|c|c|c|c|c|}
\hline Alpha Factor & 5 th\% & Mean & Median & 95 th\% & MLE & a & b \\
\hline 1 & 0.9658770 & 0.9697650 & 0.9698190 & 0.9734600 & 0.9695410 & $5.3515 \mathrm{E}+03$ & $1.6685 \mathrm{E}+02$ \\
\hline 2 & $1.02 \mathrm{E}-02$ & $1.26 \mathrm{E}-02$ & $1.25 \mathrm{E}-02$ & $1.52 \mathrm{E}-02$ & $1.26 \mathrm{E}-02$ & $6.9522 \mathrm{E}+01$ & $5.4488 \mathrm{E}+03$ \\
\hline 3 & $5.42 \mathrm{E}-03$ & $7.19 \mathrm{E}-03$ & $7.13 \mathrm{E}-03$ & $9.15 \mathrm{E}-03$ & $7.22 \mathrm{E}-03$ & $3.9655 \mathrm{E}+01$ & $5.4787 \mathrm{E}+03$ \\
\hline 4 & $4.09 \mathrm{E}-03$ & $5.64 \mathrm{E}-03$ & $5.59 \mathrm{E}-03$ & $7.40 \mathrm{E}-03$ & $5.72 \mathrm{E}-03$ & $3.1149 \mathrm{E}+01$ & $5.4872 \mathrm{E}+03$ \\
\hline 5 & $2.30 \mathrm{E}-03$ & $3.50 \mathrm{E}-03$ & $3.44 \mathrm{E}-03$ & $4.90 \mathrm{E}-03$ & $3.57 \mathrm{E}-03$ & $1.9307 \mathrm{E}+01$ & $5.4990 \mathrm{E}+03$ \\
\hline 6 & $6.22 \mathrm{E}-04$ & $1.31 \mathrm{E}-03$ & $1.25 \mathrm{E}-03$ & $2.20 \mathrm{E}-03$ & $1.34 \mathrm{E}-03$ & $7.2149 \mathrm{E}+00$ & $5.5111 \mathrm{E}+03$ \\
\hline
\end{tabular}




\section{CCF Parameter Estimations 2007, Section 3}

\section{Section 3.1.2, Generic Rate CCF Prior Distribution: CCF-RATE}

\begin{tabular}{|c|c|}
\hline Failure Mode : & SPURIOUS ACTUATION \\
& FAIL TO RUN \\
& FAIL TO REMAIN CLOSED (DETECTABLE \\
& LEAKAGE) \\
& NO VOLTAGE/AMPERAGE OUTPUT \\
& HIGH VOLTAGE/AMPERAGE OUTPUT \\
& NO FLOW/PLUGGED \\
\hline Start Date: & $1991 / 01 / 01$ \\
\hline Data Version: & $2007 / 12 / 31$ \\
\hline
\end{tabular}

Total Number of Independent Failure Events: 4161

Total Number of Common-Cause Failure Events: 270

\section{ALPHA FACTOR DISTRIBUTIONS}

$\mathrm{CCCG}=2$

\begin{tabular}{|c|c|c|c|c|c|c|c|}
\hline Alpha Factor & 5 th\% & Mean & Median & 95th\% & MLE & a & b \\
\hline 1 & 0.9654480 & 0.9721610 & 0.9723360 & 0.9782680 & 0.9721390 & $1.7231 \mathrm{E}+03$ & $4.9343 \mathrm{E}+01$ \\
\hline 2 & $2.17 \mathrm{E}-02$ & $2.78 \mathrm{E}-02$ & $2.77 \mathrm{E}-02$ & $3.46 \mathrm{E}-02$ & $2.79 \mathrm{E}-02$ & $4.9343 \mathrm{E}+01$ & $1.7231 \mathrm{E}+03$ \\
\hline
\end{tabular}

$\mathrm{CCCG}=3$

\begin{tabular}{|c|c|c|c|c|c|c|c|}
\hline Alpha Factor & 5 th\% & Mean & Median & 95 th $\%$ & MLE & a & b \\
\hline 1 & 0.9667950 & 0.9722490 & 0.9723650 & 0.9772920 & 0.9721910 & $2.5686 \mathrm{E}+03$ & $7.3317 \mathrm{E}+01$ \\
\hline 2 & $1.34 \mathrm{E}-02$ & $1.73 \mathrm{E}-02$ & $1.72 \mathrm{E}-02$ & $2.17 \mathrm{E}-02$ & $1.73 \mathrm{E}-02$ & $4.5816 \mathrm{E}+01$ & $2.5961 \mathrm{E}+03$ \\
\hline 3 & $7.39 \mathrm{E}-03$ & $1.04 \mathrm{E}-02$ & $1.03 \mathrm{E}-02$ & $1.39 \mathrm{E}-02$ & $1.05 \mathrm{E}-02$ & $2.7501 \mathrm{E}+01$ & $2.6144 \mathrm{E}+03$ \\
\hline
\end{tabular}

$\mathrm{CCCG}=4$

\begin{tabular}{|c|c|c|c|c|c|c|c|}
\hline Alpha Factor & 5 th $\%$ & Mean & Median & 95 th $\%$ & MLE & a & b \\
\hline 1 & 0.9686110 & 0.9732420 & 0.9733290 & 0.9775700 & 0.9732230 & $3.4094 \mathrm{E}+03$ & $9.3738 \mathrm{E}+01$ \\
\hline 2 & $1.07 \mathrm{E}-02$ & $1.37 \mathrm{E}-02$ & $1.36 \mathrm{E}-02$ & $1.71 \mathrm{E}-02$ & $1.36 \mathrm{E}-02$ & $4.8060 \mathrm{E}+01$ & $3.4551 \mathrm{E}+03$ \\
\hline 3 & $5.97 \mathrm{E}-03$ & $8.33 \mathrm{E}-03$ & $8.23 \mathrm{E}-03$ & $1.10 \mathrm{E}-02$ & $8.38 \mathrm{E}-03$ & $2.9168 \mathrm{E}+01$ & $3.4740 \mathrm{E}+03$ \\
\hline 4 & $2.98 \mathrm{E}-03$ & $4.71 \mathrm{E}-03$ & $4.62 \mathrm{E}-03$ & $6.76 \mathrm{E}-03$ & $4.75 \mathrm{E}-03$ & $1.6510 \mathrm{E}+01$ & $3.4866 \mathrm{E}+03$ \\
\hline
\end{tabular}

CCCG $=5$

\begin{tabular}{|c|c|c|c|c|c|c|c|}
\hline Alpha Factor & 5 th $\%$ & Mean & Median & 95 th\% & MLE & a & b \\
\hline 1 & 0.9707490 & 0.9747580 & 0.9748320 & 0.9785210 & 0.9747130 & $4.2943 \mathrm{E}+03$ & $1.1120 \mathrm{E}+02$ \\
\hline 2 & $8.72 \mathrm{E}-03$ & $1.12 \mathrm{E}-02$ & $1.11 \mathrm{E}-02$ & $1.39 \mathrm{E}-02$ & $1.11 \mathrm{E}-02$ & $4.9342 \mathrm{E}+01$ & $4.3562 \mathrm{E}+03$ \\
\hline 3 & $5.10 \mathrm{E}-03$ & $7.04 \mathrm{E}-03$ & $6.96 \mathrm{E}-03$ & $9.23 \mathrm{E}-03$ & $7.05 \mathrm{E}-03$ & $3.1009 \mathrm{E}+01$ & $4.3745 \mathrm{E}+03$ \\
\hline 4 & $3.30 \mathrm{E}-03$ & $4.89 \mathrm{E}-03$ & $4.82 \mathrm{E}-03$ & $6.74 \mathrm{E}-03$ & $4.97 \mathrm{E}-03$ & $2.1551 \mathrm{E}+01$ & $4.3840 \mathrm{E}+03$ \\
\hline 5 & $1.12 \mathrm{E}-03$ & $2.11 \mathrm{E}-03$ & $2.04 \mathrm{E}-03$ & $3.36 \mathrm{E}-03$ & $2.16 \mathrm{E}-03$ & $9.3017 \mathrm{E}+00$ & $4.3962 \mathrm{E}+03$ \\
\hline
\end{tabular}

$\mathrm{CCCG}=6$

\begin{tabular}{|c|c|c|c|c|c|c|c|}
\hline Alpha Factor & 5 th\% & Mean & Median & 95 th\% & MLE & a & b \\
\hline 1 & 0.9724430 & 0.9760120 & 0.9760750 & 0.9793740 & 0.9760020 & $5.1456 \mathrm{E}+03$ & $1.2647 \mathrm{E}+02$ \\
\hline 2 & $7.54 \mathrm{E}-03$ & $9.65 \mathrm{E}-03$ & $9.59 \mathrm{E}-03$ & $1.20 \mathrm{E}-02$ & $9.55 \mathrm{E}-03$ & $5.0864 \mathrm{E}+01$ & $5.2212 \mathrm{E}+03$ \\
\hline 3 & $4.25 \mathrm{E}-03$ & $5.87 \mathrm{E}-03$ & $5.81 \mathrm{E}-03$ & $7.70 \mathrm{E}-03$ & $5.86 \mathrm{E}-03$ & $3.0945 \mathrm{E}+01$ & $5.2411 \mathrm{E}+03$ \\
\hline 4 & $3.19 \mathrm{E}-03$ & $4.61 \mathrm{E}-03$ & $4.55 \mathrm{E}-03$ & $6.24 \mathrm{E}-03$ & $4.65 \mathrm{E}-03$ & $2.4301 \mathrm{E}+01$ & $5.2478 \mathrm{E}+03$ \\
\hline 5 & $1.69 \mathrm{E}-03$ & $2.77 \mathrm{E}-03$ & $2.71 \mathrm{E}-03$ & $4.06 \mathrm{E}-03$ & $2.82 \mathrm{E}-03$ & $1.4595 \mathrm{E}+01$ & $5.2575 \mathrm{E}+03$ \\
\hline 6 & $4.66 \mathrm{E}-04$ & $1.09 \mathrm{E}-03$ & $1.03 \mathrm{E}-03$ & $1.93 \mathrm{E}-03$ & $1.12 \mathrm{E}-03$ & $5.7620 \mathrm{E}+00$ & $5.2663 \mathrm{E}+03$ \\
\hline
\end{tabular}


CCF Parameter Estimations 2009, Section 2, No Data (Prior Only)

\section{Section 2.1.3, CCF Prior Distribution, Data Version 2007/12/31}

Data Version:

$2007 / 12 / 31$

Total Number of Independent Failure Events: 0

Total Number of Common-Cause Failure Events: 0

ALPHA FACTOR DISTRIBUTIONS

CCCG = 2
\begin{tabular}{|l|l|l|l|l|l|l|l|}
\hline Alpha Factor & 5 th\% & Mean & Median & 95 th\% & MLE & a & b \\
\hline $\mathbf{a}_{1}$ & 0.8993200 & 0.9742690 & 0.9887700 & 0.9999290 & - & $1.7418 \mathrm{E}+01$ & $4.6002 \mathrm{E}-01$ \\
\hline $\boldsymbol{\alpha}_{2}$ & $6.65 \mathrm{E}-05$ & $2.57 \mathrm{E}-02$ & $1.12 \mathrm{E}-02$ & $1.00 \mathrm{E}-01$ & -- & $4.6002 \mathrm{E}-01$ & $1.7418 \mathrm{E}+01$ \\
\hline
\end{tabular}

$\mathrm{CCCG}=3$

\begin{tabular}{|l|l|l|l|l|l|l|l|}
\hline Alpha Factor & 5 th\% & Mean & Median & 95 th\% & MLE & a & b \\
\hline $\mathbf{\alpha}_{1}$ & 0.9306240 & 0.9755060 & 0.9819700 & 0.9982830 & - & $4.5105 \mathrm{E}+01$ & $1.1325 \mathrm{E}+00$ \\
\hline $\mathbf{\alpha}_{2}$ & $6.61 \mathrm{E}-04$ & $1.87 \mathrm{E}-02$ & $1.23 \mathrm{E}-02$ & $5.84 \mathrm{E}-02$ & -- & $8.6476 \mathrm{E}-01$ & $4.5372 \mathrm{E}+01$ \\
\hline $\mathbf{\alpha}_{3}$ & $2.07 \mathrm{E}-07$ & $5.79 \mathrm{E}-03$ & $1.17 \mathrm{E}-03$ & $2.74 \mathrm{E}-02$ & -- & $2.6776 \mathrm{E}-01$ & $4.5969 \mathrm{E}+01$ \\
\hline
\end{tabular}

$\mathrm{CCCG}=4$

\begin{tabular}{|l|l|l|l|l|l|l|l|}
\hline Alpha Factor & 5 th\% & Mean & Median & 95 th\% & MLE & a & b \\
\hline $\mathbf{\alpha}_{1}$ & 0.9380870 & 0.9740820 & 0.9782970 & 0.9956540 & - & $7.0868 \mathrm{E}+01$ & $1.8856 \mathrm{E}+00$ \\
\hline $\mathbf{\alpha}_{2}$ & $1.43 \mathrm{E}-03$ & $1.70 \mathrm{E}-02$ & $1.28 \mathrm{E}-02$ & $4.69 \mathrm{E}-02$ & - & $1.2400 \mathrm{E}+00$ & $7.1513 \mathrm{E}+01$ \\
\hline $\boldsymbol{\alpha}_{3}$ & $9.66 \mathrm{E}-06$ & $5.89 \mathrm{E}-03$ & $2.32 \mathrm{E}-03$ & $2.38 \mathrm{E}-02$ & - & $4.2870 \mathrm{E}-01$ & $7.2324 \mathrm{E}+01$ \\
\hline $\mathbf{\alpha}_{4}$ & $9.21 \mathrm{E}-09$ & $2.98 \mathrm{E}-03$ & $3.83 \mathrm{E}-04$ & $1.50 \mathrm{E}-02$ & - & $2.1695 \mathrm{E}-01$ & $7.2536 \mathrm{E}+01$ \\
\hline
\end{tabular}

$\mathrm{CCCG}=5$

\begin{tabular}{|l|l|l|l|l|l|l|l|}
\hline Alpha Factor & 5 th\% & Mean & Median & 95 th\% & MLE & a & b \\
\hline $\boldsymbol{\alpha}_{1}$ & 0.9521790 & 0.9760740 & 0.9782400 & 0.9925770 & -- & $1.4106 \mathrm{E}+02$ & $3.4576 \mathrm{E}+00$ \\
\hline $\boldsymbol{\alpha}_{2}$ & $2.59 \mathrm{E}-03$ & $1.41 \mathrm{E}-02$ & $1.19 \mathrm{E}-02$ & $3.30 \mathrm{E}-02$ & -- & $2.0400 \mathrm{E}+00$ & $1.4247 \mathrm{E}+02$ \\
\hline $\boldsymbol{\alpha}_{3}$ & $3.01 \mathrm{E}-04$ & $6.59 \mathrm{E}-03$ & $4.50 \mathrm{E}-03$ & $2.00 \mathrm{E}-02$ & -- & $9.5369 \mathrm{E}-01$ & $1.4356 \mathrm{E}+02$ \\
\hline $\boldsymbol{\sigma}_{4}$ & $2.21 \mathrm{E}-06$ & $2.67 \mathrm{E}-03$ & $9.37 \mathrm{E}-04$ & $1.12 \mathrm{E}-02$ & -- & $3.8684 \mathrm{E}-01$ & $1.4413 \mathrm{E}+02$ \\
\hline $\boldsymbol{\alpha}_{5}$ & $5.61 \mathrm{E}-20$ & $5.33 \mathrm{E}-04$ & $5.18 \mathrm{E}-07$ & $3.10 \mathrm{E}-03$ & -- & $7.7129 \mathrm{E}-02$ & $1.4444 \mathrm{E}+02$ \\
\hline
\end{tabular}

$\mathrm{CCCG}=6$

\begin{tabular}{|l|l|l|l|l|l|l|l|}
\hline Alpha Factor & 5th\% & Mean & Median & 95 th\% & MLE & a & b \\
\hline $\mathbf{a}_{1}$ & 0.9553700 & 0.9762820 & 0.9779970 & 0.9913440 & -- & $1.7893 \mathrm{E}+02$ & $4.3470 \mathrm{E}+00$ \\
\hline $\mathbf{\alpha}_{2}$ & $2.60 \mathrm{E}-03$ & $1.24 \mathrm{E}-02$ & $1.07 \mathrm{E}-02$ & $2.81 \mathrm{E}-02$ & -- & $2.2804 \mathrm{E}+00$ & $1.8099 \mathrm{E}+02$ \\
\hline $\boldsymbol{\alpha}_{3}$ & $4.16 \mathrm{E}-04$ & $6.13 \mathrm{E}-03$ & $4.45 \mathrm{E}-03$ & $1.75 \mathrm{E}-02$ & - & $1.1245 \mathrm{E}+00$ & $1.8215 \mathrm{E}+02$ \\
\hline $\mathbf{\alpha}_{4}$ & $3.82 \mathrm{E}-05$ & $3.40 \mathrm{E}-03$ & $1.85 \mathrm{E}-03$ & $1.20 \mathrm{E}-02$ & - & $6.2471 \mathrm{E}-01$ & $1.8265 \mathrm{E}+02$ \\
\hline $\mathbf{\alpha}_{5}$ & $1.60 \mathrm{E}-08$ & $1.32 \mathrm{E}-03$ & $2.18 \mathrm{E}-04$ & $6.46 \mathrm{E}-03$ & -- & $2.4272 \mathrm{E}-01$ & $1.8303 \mathrm{E}+02$ \\
\hline $\mathbf{a}_{6}$ & $1.26 \mathrm{E}-20$ & $4.07 \mathrm{E}-04$ & $3.05 \mathrm{E}-07$ & $2.36 \mathrm{E}-03$ & -- & $7.4722 \mathrm{E}-02$ & $1.8320 \mathrm{E}+02$ \\
\hline
\end{tabular}

$\mathrm{CCCG}=7$

\begin{tabular}{|l|l|l|l|l|l|l|l|}
\hline Alpha Factor & 5th\% & Mean & Median & 95 th\% & MLE & a & b \\
\hline $\mathbf{\alpha}_{1}$ & 0.9603690 & 0.9769760 & 0.9781320 & 0.9896440 & -- & $2.6720 \mathrm{E}+02$ & $6.2971 \mathrm{E}+00$ \\
\hline $\boldsymbol{\alpha}_{2}$ & $3.14 \mathrm{E}-03$ & $1.12 \mathrm{E}-02$ & $1.00 \mathrm{E}-02$ & $2.33 \mathrm{E}-02$ & -- & $3.0721 \mathrm{E}+00$ & $2.7042 \mathrm{E}+02$ \\
\hline $\boldsymbol{\alpha}_{3}$ & $6.66 \mathrm{E}-04$ & $5.55 \mathrm{E}-03$ & $4.40 \mathrm{E}-03$ & $1.43 \mathrm{E}-02$ & - & $1.5182 \mathrm{E}+00$ & $2.7197 \mathrm{E}+02$ \\
\hline $\boldsymbol{\alpha}_{4}$ & $1.58 \mathrm{E}-04$ & $3.48 \mathrm{E}-03$ & $2.37 \mathrm{E}-03$ & $1.06 \mathrm{E}-02$ & -- & $9.5310 \mathrm{E}-01$ & $2.7254 \mathrm{E}+02$ \\
\hline $\boldsymbol{\alpha}_{5}$ & $1.00 \mathrm{E}-05$ & $1.93 \mathrm{E}-03$ & $9.22 \mathrm{E}-04$ & $7.26 \mathrm{E}-03$ & -- & $5.2795 \mathrm{E}-01$ & $2.7296 \mathrm{E}+02$ \\
\hline $\mathbf{\alpha}_{6}$ & $4.58 \mathrm{E}-10$ & $7.08 \mathrm{E}-04$ & $6.75 \mathrm{E}-05$ & $3.68 \mathrm{E}-03$ & -- & $1.9373 \mathrm{E}-01$ & $2.7330 \mathrm{E}+02$ \\
\hline $\boldsymbol{\alpha}_{7}$ & $5.03 \mathrm{E}-44$ & $1.17 \mathrm{E}-04$ & $8.41 \mathrm{E}-13$ & $4.81 \mathrm{E}-04$ & - & $3.2027 \mathrm{E}-02$ & $2.7346 \mathrm{E}+02$ \\
\hline
\end{tabular}

$\mathrm{CCCG}=8$

\begin{tabular}{|l|l|l|l|l|l|l|l|}
\hline Alpha Factor & 5 th\% & Mean & Median & 95 th\% & MLE & a & b \\
\hline $\mathbf{\alpha}_{1}$ & 0.9622170 & 0.9773660 & 0.9783580 & 0.9891370 & - & $3.1221 \mathrm{E}+02$ & $7.2302 \mathrm{E}+00$ \\
\hline $\mathbf{\alpha}_{2}$ & $3.13 \mathrm{E}-03$ & $1.04 \mathrm{E}-02$ & $9.45 \mathrm{E}-03$ & $2.12 \mathrm{E}-02$ & - & $3.3414 \mathrm{E}+00$ & $3.1609 \mathrm{E}+02$ \\
\hline $\mathbf{\alpha}_{3}$ & $6.67 \mathrm{E}-04$ & $5.04 \mathrm{E}-03$ & $4.06 \mathrm{E}-03$ & $1.28 \mathrm{E}-02$ & -- & $1.6130 \mathrm{E}+00$ & $3.1782 \mathrm{E}+02$ \\
\hline $\mathbf{\alpha}_{4}$ & $1.86 \mathrm{E}-04$ & $3.26 \mathrm{E}-03$ & $2.30 \mathrm{E}-03$ & $9.62 \mathrm{E}-03$ & - & $1.0438 \mathrm{E}+00$ & $3.1839 \mathrm{E}+02$ \\
\hline $\mathbf{\alpha}_{5}$ & $3.88 \mathrm{E}-05$ & $2.20 \mathrm{E}-03$ & $1.28 \mathrm{E}-03$ & $7.47 \mathrm{E}-03$ & -- & $7.0280 \mathrm{E}-01$ & $3.1873 \mathrm{E}+02$ \\
\hline $\mathbf{\alpha}_{6}$ & $5.77 \mathrm{E}-07$ & $1.13 \mathrm{E}-03$ & $3.63 \mathrm{E}-04$ & $4.86 \mathrm{E}-03$ & -- & $3.6184 \mathrm{E}-01$ & $3.1907 \mathrm{E}+02$ \\
\hline $\mathbf{\alpha}_{7}$ & $1.19 \mathrm{E}-13$ & $3.98 \mathrm{E}-04$ & $8.44 \mathrm{E}-06$ & $2.25 \mathrm{E}-03$ & - & $1.2739 \mathrm{E}-01$ & $3.1931 \mathrm{E}+02$ \\
\hline $\mathbf{\alpha}_{8}$ & $5.47 \mathrm{E}-36$ & $1.25 \mathrm{E}-04$ & $5.43 \mathrm{E}-11$ & $6.01 \mathrm{E}-04$ & -- & $4.0005 \mathrm{E}-02$ & $3.1940 \mathrm{E}+02$ \\
\hline
\end{tabular}




\section{CCF Parameter Estimations 2009, Section 2}

\section{Section 2.1.1, Generic Demand CCF Prior Distribution: CCF-DEM}

Failure Mode :

Fail to close (reseat) on demand

Fail to open on demand

Fail to start

Fail to Operate (Open/Close)

Start Date :

Fail to stop

Data Version :

1997/01/01

2009/12/31

Total Number of Independent Failure Events: 2446.50

Total Number of Common-Cause Failure Events: 97

ALPHA FACTOR DISTRIBUTIONS

CCCG $=2$

\begin{tabular}{|l|l|l|l|l|l|l|l|}
\hline Alpha Factor & 5th\% & Mean & Median & 95 th\% & MLE & a & b \\
\hline $\mathbf{a}_{1}$ & 0.9634050 & 0.9726920 & 0.9730300 & 0.9808300 & 0.9728470 & $9.1215 \mathrm{E}+02$ & $2.5608 \mathrm{E}+01$ \\
\hline $\mathbf{\alpha}_{2}$ & $1.92 \mathrm{E}-02$ & $2.73 \mathrm{E}-02$ & $2.70 \mathrm{E}-02$ & $3.66 \mathrm{E}-02$ & $2.72 \mathrm{E}-02$ & $2.5608 \mathrm{E}+01$ & $9.1215 \mathrm{E}+02$ \\
\hline
\end{tabular}

$\mathrm{CCCG}=3$

\begin{tabular}{|l|l|l|l|l|l|l|l|}
\hline Alpha Factor & 5th\% & Mean & Median & 95 th\% & MLE & a & b \\
\hline $\mathbf{a}_{1}$ & 0.9656500 & 0.9731230 & 0.9733470 & 0.9798310 & 0.9733270 & $1.3609 \mathrm{E}+03$ & $3.7587 \mathrm{E}+01$ \\
\hline $\mathbf{\alpha}_{2}$ & $1.12 \mathrm{E}-02$ & $1.63 \mathrm{E}-02$ & $1.61 \mathrm{E}-02$ & $2.22 \mathrm{E}-02$ & $1.61 \mathrm{E}-02$ & $2.2803 \mathrm{E}+01$ & $1.3757 \mathrm{E}+03$ \\
\hline $\mathbf{\alpha}_{3}$ & $6.51 \mathrm{E}-03$ & $1.06 \mathrm{E}-02$ & $1.03 \mathrm{E}-02$ & $1.54 \mathrm{E}-02$ & $1.06 \mathrm{E}-02$ & $1.4784 \mathrm{E}+01$ & $1.3837 \mathrm{E}+03$ \\
\hline
\end{tabular}

$\mathrm{CCCG}=4$

\begin{tabular}{|l|l|l|l|l|l|l|l|}
\hline Alpha Factor & 5 th\% & Mean & Median & 95 th\% & MLE & a & b \\
\hline $\mathbf{\alpha}_{1}$ & 0.9663450 & 0.9728260 & 0.9729930 & 0.9787330 & 0.9731310 & $1.8052 \mathrm{E}+03$ & $5.0425 \mathrm{E}+01$ \\
\hline $\boldsymbol{\alpha}_{2}$ & $1.12 \mathrm{E}-02$ & $1.56 \mathrm{E}-02$ & $1.54 \mathrm{E}-02$ & $2.06 \mathrm{E}-02$ & $1.53 \mathrm{E}-02$ & $2.8905 \mathrm{E}+01$ & $1.8267 \mathrm{E}+03$ \\
\hline $\boldsymbol{\alpha}_{3}$ & $3.63 \mathrm{E}-03$ & $6.33 \mathrm{E}-03$ & $6.15 \mathrm{E}-03$ & $9.62 \mathrm{E}-03$ & $6.27 \mathrm{E}-03$ & $1.1737 \mathrm{E}+01$ & $1.8439 \mathrm{E}+03$ \\
\hline $\boldsymbol{\alpha}_{4}$ & $2.84 \mathrm{E}-03$ & $5.27 \mathrm{E}-03$ & $5.10 \mathrm{E}-03$ & $8.31 \mathrm{E}-03$ & $5.29 \mathrm{E}-03$ & $9.7833 \mathrm{E}+00$ & $1.8458 \mathrm{E}+03$ \\
\hline
\end{tabular}

$\mathrm{CCCG}=5$

\begin{tabular}{|l|l|l|l|l|l|l|l|}
\hline Alpha Factor & 5 th\% & Mean & Median & 95 th\% & MLE & a & b \\
\hline $\mathbf{\alpha}_{1}$ & 0.9696730 & 0.9751830 & 0.9753210 & 0.9802270 & 0.9756580 & $2.2837 \mathrm{E}+03$ & $5.8116 \mathrm{E}+01$ \\
\hline $\boldsymbol{\alpha}_{2}$ & $8.25 \mathrm{E}-03$ & $1.16 \mathrm{E}-02$ & $1.15 \mathrm{E}-02$ & $1.55 \mathrm{E}-02$ & $1.12 \mathrm{E}-02$ & $2.7259 \mathrm{E}+01$ & $2.3146 \mathrm{E}+03$ \\
\hline $\boldsymbol{\alpha}_{3}$ & $4.07 \mathrm{E}-03$ & $6.55 \mathrm{E}-03$ & $6.41 \mathrm{E}-03$ & $9.51 \mathrm{E}-03$ & $6.40 \mathrm{E}-03$ & $1.5335 \mathrm{E}+01$ & $2.3265 \mathrm{E}+03$ \\
\hline $\mathbf{\alpha}_{4}$ & $1.75 \mathrm{E}-03$ & $3.49 \mathrm{E}-03$ & $3.35 \mathrm{E}-03$ & $5.70 \mathrm{E}-03$ & $3.47 \mathrm{E}-03$ & $8.1629 \mathrm{E}+00$ & $2.3337 \mathrm{E}+03$ \\
\hline $\mathbf{\alpha}_{5}$ & $1.51 \mathrm{E}-03$ & $3.14 \mathrm{E}-03$ & $3.00 \mathrm{E}-03$ & $5.25 \mathrm{E}-03$ & $3.25 \mathrm{E}-03$ & $7.3588 \mathrm{E}+00$ & $2.3345 \mathrm{E}+03$ \\
\hline
\end{tabular}

$\mathrm{CCCG}=6$

\begin{tabular}{|l|l|l|l|l|l|l|l|}
\hline Alpha Factor & 5 th\% & Mean & Median & 95 th\% & MLE & a & b \\
\hline $\mathbf{\alpha}_{1}$ & 0.9724050 & 0.9772250 & 0.9773410 & 0.9816580 & 0.9777920 & $2.7361 \mathrm{E}+03$ & $6.3768 \mathrm{E}+01$ \\
\hline $\mathbf{\alpha}_{2}$ & $6.22 \mathrm{E}-03$ & $8.93 \mathrm{E}-03$ & $8.81 \mathrm{E}-03$ & $1.20 \mathrm{E}-02$ & $8.50 \mathrm{E}-03$ & $2.5000 \mathrm{E}+01$ & $2.7749 \mathrm{E}+03$ \\
\hline $\boldsymbol{\alpha}_{3}$ & $3.92 \mathrm{E}-03$ & $6.13 \mathrm{E}-03$ & $6.01 \mathrm{E}-03$ & $8.74 \mathrm{E}-03$ & $5.98 \mathrm{E}-03$ & $1.7153 \mathrm{E}+01$ & $2.7827 \mathrm{E}+03$ \\
\hline $\mathbf{\alpha}_{4}$ & $1.94 \mathrm{E}-03$ & $3.57 \mathrm{E}-03$ & $3.45 \mathrm{E}-03$ & $5.60 \mathrm{E}-03$ & $3.51 \mathrm{E}-03$ & $9.9848 \mathrm{E}+00$ & $2.7899 \mathrm{E}+03$ \\
\hline $\mathbf{\alpha}_{5}$ & $7.95 \mathrm{E}-04$ & $1.93 \mathrm{E}-03$ & $1.81 \mathrm{E}-03$ & $3.46 \mathrm{E}-03$ & $1.93 \mathrm{E}-03$ & $5.4037 \mathrm{E}+00$ & $2.7945 \mathrm{E}+03$ \\
\hline $\mathbf{\alpha}_{5}$ & $9.88 \mathrm{E}-04$ & $2.22 \mathrm{E}-03$ & $2.11 \mathrm{E}-03$ & $3.86 \mathrm{E}-03$ & $2.29 \mathrm{E}-03$ & $6.2261 \mathrm{E}+00$ & $2.7936 \mathrm{E}+03$ \\
\hline
\end{tabular}




\section{CCF Parameter Estimations 2009, Section 2}

\section{Section 2.1.2, Generic Rate CCF Prior Distribution: CCF-RATE}

Failure Mode :

Start Date

Data Version :

\author{
Spurious operation open or close \\ Fail to run \\ Filter media allows the pass through of debris \\ High dP across filter \\ Fail to control flow \\ High voltage/ amperage output \\ Loss of heat transfer capabilities in heat exchangers \\ No voltage/amperage output \\ No flow/plugged \\ 1997/01/01 \\ 2009/12/31
}

Total Number of Independent Failure Events: 1857.60

Total Number of Common-Cause Failure Events: 67

\section{ALPHA FACTOR DISTRIBUTIONS}

$C C C G=2$

\begin{tabular}{|l|l|l|l|l|l|l|l|}
\hline Alpha Factor & 5 th\% & Mean & Median & 95 th\% & MLE & a & b \\
\hline $\mathbf{a}_{1}$ & 0.9641020 & 0.9733720 & 0.9737170 & 0.9814740 & 0.9735380 & $8.9523 \mathrm{E}+02$ & $2.4490 \mathrm{E}+01$ \\
\hline $\mathbf{a}_{2}$ & $1.85 \mathrm{E}-02$ & $2.66 \mathrm{E}-02$ & $2.63 \mathrm{E}-02$ & $3.59 \mathrm{E}-02$ & $2.65 \mathrm{E}-02$ & $2.4490 \mathrm{E}+01$ & $8.9523 \mathrm{E}+02$ \\
\hline
\end{tabular}

$\mathrm{CCCG}=3$

\begin{tabular}{|l|l|l|l|l|l|l|l|}
\hline Alpha Factor & 5th\% & Mean & Median & 95 th\% & MLE & a & b \\
\hline $\mathbf{a}_{1}$ & 0.9668950 & 0.9742880 & 0.9745210 & 0.9808970 & 0.9745220 & $1.3372 \mathrm{E}+03$ & $3.5289 \mathrm{E}+01$ \\
\hline $\mathbf{\alpha}_{2}$ & $1.00 \mathrm{E}-02$ & $1.50 \mathrm{E}-02$ & $1.47 \mathrm{E}-02$ & $2.07 \mathrm{E}-02$ & $1.47 \mathrm{E}-02$ & $2.0532 \mathrm{E}+01$ & $1.3520 \mathrm{E}+03$ \\
\hline $\mathbf{\alpha}_{3}$ & $6.61 \mathrm{E}-03$ & $1.08 \mathrm{E}-02$ & $1.05 \mathrm{E}-02$ & $1.57 \mathrm{E}-02$ & $1.08 \mathrm{E}-02$ & $1.4757 \mathrm{E}+01$ & $1.3577 \mathrm{E}+03$ \\
\hline
\end{tabular}

$\mathrm{CCCG}=4$

\begin{tabular}{|l|l|l|l|l|l|l|l|}
\hline Alpha Factor & 5 th\% & Mean & Median & 95 th\% & MLE & a & b \\
\hline $\mathbf{\alpha}_{1}$ & 0.9702030 & 0.9763400 & 0.9765110 & 0.9818840 & 0.9767450 & $1.7802 \mathrm{E}+03$ & $4.3139 \mathrm{E}+01$ \\
\hline $\mathbf{\alpha}_{2}$ & $7.13 \mathrm{E}-03$ & $1.08 \mathrm{E}-02$ & $1.06 \mathrm{E}-02$ & $1.50 \mathrm{E}-02$ & $1.04 \mathrm{E}-02$ & $1.9652 \mathrm{E}+01$ & $1.8037 \mathrm{E}+03$ \\
\hline $\mathbf{\alpha}_{3}$ & $5.26 \mathrm{E}-03$ & $8.45 \mathrm{E}-03$ & $8.27 \mathrm{E}-03$ & $1.23 \mathrm{E}-02$ & $8.45 \mathrm{E}-03$ & $1.5406 \mathrm{E}+01$ & $1.8079 \mathrm{E}+03$ \\
\hline $\mathbf{\alpha}_{4}$ & $2.22 \mathrm{E}-03$ & $4.43 \mathrm{E}-03$ & $4.25 \mathrm{E}-03$ & $7.26 \mathrm{E}-03$ & $4.43 \mathrm{E}-03$ & $8.0813 \mathrm{E}+00$ & $1.8153 \mathrm{E}+03$ \\
\hline
\end{tabular}

$\mathrm{CCCG}=5$

\begin{tabular}{|l|l|l|l|l|l|l|l|}
\hline Alpha Factor & 5th\% & Mean & Median & 95 th\% & MLE & a & b \\
\hline $\mathbf{a}_{1}$ & 0.9736030 & 0.9787670 & 0.9789070 & 0.9834530 & 0.9794110 & $2.2573 \mathrm{E}+03$ & $4.8969 \mathrm{E}+01$ \\
\hline $\mathbf{\alpha}_{2}$ & $4.92 \mathrm{E}-03$ & $7.64 \mathrm{E}-03$ & $7.50 \mathrm{E}-03$ & $1.08 \mathrm{E}-02$ & $7.02 \mathrm{E}-03$ & $1.7615 \mathrm{E}+01$ & $2.2887 \mathrm{E}+03$ \\
\hline $\mathbf{\alpha} 3$ & $4.25 \mathrm{E}-03$ & $6.81 \mathrm{E}-03$ & $6.66 \mathrm{E}-03$ & $9.84 \mathrm{E}-03$ & $6.67 \mathrm{E}-03$ & $1.5698 \mathrm{E}+01$ & $2.2906 \mathrm{E}+03$ \\
\hline $\mathbf{\alpha} 4$ & $2.74 \mathrm{E}-03$ & $4.85 \mathrm{E}-03$ & $4.71 \mathrm{E}-03$ & $7.45 \mathrm{E}-03$ & $4.90 \mathrm{E}-03$ & $1.1185 \mathrm{E}+01$ & $2.2951 \mathrm{E}+03$ \\
\hline $\mathbf{\alpha} 5$ & $7.14 \mathrm{E}-04$ & $1.94 \mathrm{E}-03$ & $1.80 \mathrm{E}-03$ & $3.65 \mathrm{E}-03$ & $1.99 \mathrm{E}-03$ & $4.4708 \mathrm{E}+00$ & $2.3018 \mathrm{E}+03$ \\
\hline
\end{tabular}

$\mathrm{CCCG}=6$

\begin{tabular}{|l|l|l|l|l|l|l|l|}
\hline Alpha Factor & 5 th\% & Mean & Median & 95 th\% & MLE & a & b \\
\hline $\mathbf{\alpha}_{1}$ & 0.9762820 & 0.9807670 & 0.9808860 & 0.9848620 & 0.9815060 & $2.7062 \mathrm{E}+03$ & $5.3068 \mathrm{E}+01$ \\
\hline $\mathbf{\alpha}_{2}$ & $3.50 \mathrm{E}-03$ & $5.62 \mathrm{E}-03$ & $5.50 \mathrm{E}-03$ & $8.15 \mathrm{E}-03$ & $5.03 \mathrm{E}-03$ & $1.5513 \mathrm{E}+01$ & $2.7438 \mathrm{E}+03$ \\
\hline $\mathbf{\alpha}_{3}$ & $3.30 \mathrm{E}-03$ & $5.37 \mathrm{E}-03$ & $5.25 \mathrm{E}-03$ & $7.84 \mathrm{E}-03$ & $5.18 \mathrm{E}-03$ & $1.4806 \mathrm{E}+01$ & $2.7445 \mathrm{E}+03$ \\
\hline $\mathbf{\alpha}_{4}$ & $2.71 \mathrm{E}-03$ & $4.61 \mathrm{E}-03$ & $4.49 \mathrm{E}-03$ & $6.92 \mathrm{E}-03$ & $4.60 \mathrm{E}-03$ & $1.2730 \mathrm{E}+01$ & $2.7465 \mathrm{E}+03$ \\
\hline $\mathrm{a}_{5}$ & $1.24 \mathrm{E}-03$ & $2.61 \mathrm{E}-03$ & $2.49 \mathrm{E}-03$ & $4.39 \mathrm{E}-03$ & $2.65 \mathrm{E}-03$ & $7.2117 \mathrm{E}+00$ & $2.7521 \mathrm{E}+03$ \\
\hline $\mathbf{\alpha}_{6}$ & $2.61 \mathrm{E}-04$ & $1.02 \mathrm{E}-03$ & $9.00 \mathrm{E}-04$ & $2.18 \mathrm{E}-03$ & $1.03 \mathrm{E}-03$ & $2.8070 \mathrm{E}+00$ & $2.7565 \mathrm{E}+03$ \\
\hline
\end{tabular}


CCF Parameter Estimations 2010, Section 2, No Data (Prior Only)

\section{Section 2.2.3, CCF Prior Distribution, Data Version 2007/12/31}

Data Version :

$2007 / 12 / 31$

Total Number of Independent Failure Events: 0

Total Number of Common-Cause Failure Events: 0

ALPHA FACTOR DISTRIBUTIONS

CCCG $=2$

\begin{tabular}{|l|l|l|l|l|l|l|l|}
\hline Alpha Factor & 5 th $\%$ & Mean & Median & 95 th $\%$ & MLE & a & b \\
\hline $\boldsymbol{a}_{1}$ & 0.8993200 & 0.9742690 & 0.9887700 & 0.9999290 & -- & $1.7418 \mathrm{E}+01$ & $4.6002 \mathrm{E}-01$ \\
\hline $\mathbf{a}_{2}$ & $6.65 \mathrm{E}-05$ & $2.57 \mathrm{E}-02$ & $1.12 \mathrm{E}-02$ & $1.00 \mathrm{E}-01$ & -- & $4.6002 \mathrm{E}-01$ & $1.7418 \mathrm{E}+01$ \\
\hline
\end{tabular}

CCCG $=3$

\begin{tabular}{|l|l|l|l|l|l|l|l|}
\hline Alpha Factor & 5 th $\%$ & Mean & Median & 95 th $\%$ & MLE & $\mathbf{a}$ & $\mathbf{b}$ \\
\hline $\boldsymbol{\alpha}_{1}$ & 0.9306240 & 0.9755060 & 0.9819700 & 0.9982830 & -- & $4.5105 \mathrm{E}+01$ & $1.1325 \mathrm{E}+00$ \\
\hline $\boldsymbol{\alpha}_{2}$ & $6.61 \mathrm{E}-04$ & $1.87 \mathrm{E}-02$ & $1.23 \mathrm{E}-02$ & $5.84 \mathrm{E}-02$ & -- & $8.6476 \mathrm{E}-01$ & $4.5372 \mathrm{E}+01$ \\
\hline $\boldsymbol{\alpha}_{3}$ & $2.07 \mathrm{E}-07$ & $5.79 \mathrm{E}-03$ & $1.17 \mathrm{E}-03$ & $2.74 \mathrm{E}-02$ & -- & $2.6776 \mathrm{E}-01$ & $4.5969 \mathrm{E}+01$ \\
\hline
\end{tabular}

CCCG $=4$

\begin{tabular}{|l|l|l|l|l|l|l|l|}
\hline Alpha Factor & 5 th $\%$ & Mean & Median & 95 th $\%$ & MLE & $\mathbf{a}$ & $\mathbf{b}$ \\
\hline $\mathbf{a}_{1}$ & 0.9380870 & 0.9740820 & 0.9782970 & 0.9956540 & --- & $7.0868 \mathrm{E}+01$ & $1.8856 \mathrm{E}+00$ \\
\hline $\mathbf{a}_{2}$ & $1.43 \mathrm{E}-03$ & $1.70 \mathrm{E}-02$ & $1.28 \mathrm{E}-02$ & $4.69 \mathrm{E}-02$ & -- & $1.2400 \mathrm{E}+00$ & $7.1513 \mathrm{E}+01$ \\
\hline $\mathbf{a}_{3}$ & $9.66 \mathrm{E}-06$ & $5.89 \mathrm{E}-03$ & $2.32 \mathrm{E}-03$ & $2.38 \mathrm{E}-02$ & -- & $4.2870 \mathrm{E}-01$ & $7.2324 \mathrm{E}+01$ \\
\hline $\mathbf{a}_{4}$ & $9.21 \mathrm{E}-09$ & $2.98 \mathrm{E}-03$ & $3.83 \mathrm{E}-04$ & $1.50 \mathrm{E}-02$ & --- & $2.1695 \mathrm{E}-01$ & $7.2536 \mathrm{E}+01$ \\
\hline
\end{tabular}

CCCG $=5$

\begin{tabular}{|l|l|l|l|l|l|l|l|}
\hline Alpha Factor & 5 th $\%$ & Mean & Median & 95 th $\%$ & MLE & $\mathbf{a}$ & $\mathbf{b}$ \\
\hline $\mathbf{a}_{1}$ & 0.9521790 & 0.9760740 & 0.9782400 & 0.9925770 & -- & $1.4106 \mathrm{E}+02$ & $3.4576 \mathrm{E}+00$ \\
\hline $\boldsymbol{a}_{2}$ & $2.59 \mathrm{E}-03$ & $1.41 \mathrm{E}-02$ & $1.19 \mathrm{E}-02$ & $3.30 \mathrm{E}-02$ & --- & $2.0400 \mathrm{E}+00$ & $1.4247 \mathrm{E}+02$ \\
\hline $\boldsymbol{a}_{3}$ & $3.01 \mathrm{E}-04$ & $6.59 \mathrm{E}-03$ & $4.50 \mathrm{E}-03$ & $2.00 \mathrm{E}-02$ & -- & $9.5369 \mathrm{E}-01$ & $1.4356 \mathrm{E}+02$ \\
\hline $\mathbf{a}_{4}$ & $2.21 \mathrm{E}-06$ & $2.67 \mathrm{E}-03$ & $9.37 \mathrm{E}-04$ & $1.12 \mathrm{E}-02$ & -- & $3.8684 \mathrm{E}-01$ & $1.4413 \mathrm{E}+02$ \\
\hline $\mathbf{a}_{5}$ & $5.61 \mathrm{E}-20$ & $5.33 \mathrm{E}-04$ & $5.18 \mathrm{E}-07$ & $3.10 \mathrm{E}-03$ & --- & $7.7129 \mathrm{E}-02$ & $1.4444 \mathrm{E}+02$ \\
\hline
\end{tabular}

CCCG $=6$

\begin{tabular}{|l|l|l|l|l|l|l|l|}
\hline Alpha Factor & 5 th $\%$ & Mean & Median & 95 th $\%$ & MLE & a & b \\
\hline $\boldsymbol{\alpha}_{1}$ & 0.9553700 & 0.9762820 & 0.9779970 & 0.9913440 & -- & $1.7893 \mathrm{E}+02$ & $4.3470 \mathrm{E}+00$ \\
\hline $\boldsymbol{\alpha}_{2}$ & $2.60 \mathrm{E}-03$ & $1.24 \mathrm{E}-02$ & $1.07 \mathrm{E}-02$ & $2.81 \mathrm{E}-02$ & -- & $2.2804 \mathrm{E}+00$ & $1.8099 \mathrm{E}+02$ \\
\hline $\boldsymbol{\alpha}_{3}$ & $4.16 \mathrm{E}-04$ & $6.13 \mathrm{E}-03$ & $4.45 \mathrm{E}-03$ & $1.75 \mathrm{E}-02$ & -- & $1.1245 \mathrm{E}+00$ & $1.8215 \mathrm{E}+02$ \\
\hline $\boldsymbol{a}_{4}$ & $3.82 \mathrm{E}-05$ & $3.40 \mathrm{E}-03$ & $1.85 \mathrm{E}-03$ & $1.20 \mathrm{E}-02$ & -- & $6.2471 \mathrm{E}-01$ & $1.8265 \mathrm{E}+02$ \\
\hline $\boldsymbol{\alpha}_{5}$ & $1.60 \mathrm{E}-08$ & $1.32 \mathrm{E}-03$ & $2.18 \mathrm{E}-04$ & $6.46 \mathrm{E}-03$ & -- & $2.4272 \mathrm{E}-01$ & $1.8303 \mathrm{E}+02$ \\
\hline $\boldsymbol{\alpha}_{6}$ & $1.26 \mathrm{E}-20$ & $4.07 \mathrm{E}-04$ & $3.05 \mathrm{E}-07$ & $2.36 \mathrm{E}-03$ & -- & $7.4722 \mathrm{E}-02$ & $1.8320 \mathrm{E}+02$ \\
\hline
\end{tabular}

CCCG $=7$

\begin{tabular}{|l|l|l|l|l|l|l|l|}
\hline Alpha Factor & 5 th $\%$ & Mean & Median & 95 th $\%$ & MLE & $\mathbf{a}$ & $\mathbf{b}$ \\
\hline $\mathbf{a}_{1}$ & 0.9603690 & 0.9769760 & 0.9781320 & 0.9896440 & -- & $2.6720 \mathrm{E}+02$ & $6.2971 \mathrm{E}+00$ \\
\hline $\mathbf{a}_{2}$ & $3.14 \mathrm{E}-03$ & $1.12 \mathrm{E}-02$ & $1.00 \mathrm{E}-02$ & $2.33 \mathrm{E}-02$ & -- & $3.0721 \mathrm{E}+00$ & $2.7042 \mathrm{E}+02$ \\
\hline $\mathbf{a}_{3}$ & $6.66 \mathrm{E}-04$ & $5.55 \mathrm{E}-03$ & $4.40 \mathrm{E}-03$ & $1.43 \mathrm{E}-02$ & -- & $1.5182 \mathrm{E}+00$ & $2.7197 \mathrm{E}+02$ \\
\hline $\mathbf{a}_{4}$ & $1.58 \mathrm{E}-04$ & $3.48 \mathrm{E}-03$ & $2.37 \mathrm{E}-03$ & $1.06 \mathrm{E}-02$ & -- & $9.5310 \mathrm{E}-01$ & $2.7254 \mathrm{E}+02$ \\
\hline $\mathbf{a}_{5}$ & $1.00 \mathrm{E}-05$ & $1.93 \mathrm{E}-03$ & $9.22 \mathrm{E}-04$ & $7.26 \mathrm{E}-03$ & -- & $5.2795 \mathrm{E}-01$ & $2.7296 \mathrm{E}+02$ \\
\hline $\mathbf{a}_{6}$ & $4.58 \mathrm{E}-10$ & $7.08 \mathrm{E}-04$ & $6.75 \mathrm{E}-05$ & $3.68 \mathrm{E}-03$ & -- & $1.9373 \mathrm{E}-01$ & $2.7330 \mathrm{E}+02$ \\
\hline $\mathbf{a}_{7}$ & $5.03 \mathrm{E}-44$ & $1.17 \mathrm{E}-04$ & $8.41 \mathrm{E}-13$ & $4.81 \mathrm{E}-04$ & -- & $3.2027 \mathrm{E}-02$ & $2.7346 \mathrm{E}+02$ \\
\hline
\end{tabular}

\section{CCCG $=8$}

\begin{tabular}{|l|l|l|l|l|l|l|l|}
\hline Alpha Factor & th $\%$ & Mean & Median & 95 th $\%$ & MLE & $\mathbf{a}$ & $\mathbf{b}$ \\
\hline $\mathbf{a}_{1}$ & 0.9622170 & 0.9773660 & 0.9783580 & 0.9891370 & -- & $3.1221 \mathrm{E}+02$ & $7.2302 \mathrm{E}+00$ \\
\hline $\mathbf{a}_{2}$ & $3.13 \mathrm{E}-03$ & $1.04 \mathrm{E}-02$ & $9.45 \mathrm{E}-03$ & $2.12 \mathrm{E}-02$ & -- & $3.3414 \mathrm{E}+00$ & $3.1609 \mathrm{E}+02$ \\
\hline $\mathbf{a}_{3}$ & $6.67 \mathrm{E}-04$ & $5.04 \mathrm{E}-03$ & $4.06 \mathrm{E}-03$ & $1.28 \mathrm{E}-02$ & -- & $1.6130 \mathrm{E}+00$ & $3.1782 \mathrm{E}+02$ \\
\hline $\mathbf{a}_{4}$ & $1.86 \mathrm{E}-04$ & $3.26 \mathrm{E}-03$ & $2.30 \mathrm{E}-03$ & $9.62 \mathrm{E}-03$ & -- & $1.0438 \mathrm{E}+00$ & $3.1839 \mathrm{E}+02$ \\
\hline $\mathbf{a}_{5}$ & $3.88 \mathrm{E}-05$ & $2.20 \mathrm{E}-03$ & $1.28 \mathrm{E}-03$ & $7.47 \mathrm{E}-03$ & -- & $7.0280 \mathrm{E}-01$ & $3.1873 \mathrm{E}+02$ \\
\hline $\mathbf{a}_{6}$ & $5.77 \mathrm{E}-07$ & $1.13 \mathrm{E}-03$ & $3.63 \mathrm{E}-04$ & $4.86 \mathrm{E}-03$ & -- & $3.6184 \mathrm{E}-01$ & $3.1907 \mathrm{E}+02$ \\
\hline $\mathbf{a}_{7}$ & $1.19 \mathrm{E}-13$ & $3.98 \mathrm{E}-04$ & $8.44 \mathrm{E}-06$ & $2.25 \mathrm{E}-03$ & -- & $1.2739 \mathrm{E}-01$ & $3.1931 \mathrm{E}+02$ \\
\hline $\mathbf{a}_{8}$ & $5.47 \mathrm{E}-36$ & $1.25 \mathrm{E}-04$ & $5.43 \mathrm{E}-11$ & $6.01 \mathrm{E}-04$ & -- & $4.0005 \mathrm{E}-02$ & $3.1940 \mathrm{E}+02$ \\
\hline
\end{tabular}




\section{CCF Parameter Estimations 2010, Section 2}

\section{Section 2.2.1, Generic Demand CCF Prior Distribution: CCF-DEM}

Failure Mode

Start Date :

Data Version :
Fail to close (reseat) on demand

Fail to Open/Close Mode Unspecified (demand based)

Fail to open on demand

Fail to start

Fail to Load/Run

Fail to stop

1997/01/01

$2010 / 12 / 31$

Total Number of Independent Failure Events: 2821.80

Total Number of Common-Cause Failure Events: 95

\section{ALPHA FACTOR DISTRIBUTIONS}

$C C C G=2$

\begin{tabular}{|l|l|l|l|l|l|l|l|}
\hline Alpha Factor & 5 th $\%$ & Mean & Median & 95 th $\%$ & MLE & a & b \\
\hline $\mathbf{\alpha}_{1}$ & 0.9679280 & 0.9760020 & 0.9762900 & 0.9830830 & 0.9761660 & $1.0674 \mathrm{E}+03$ & $2.6245 \mathrm{E}+01$ \\
\hline $\mathbf{\alpha}_{2}$ & $1.69 \mathrm{E}-02$ & $2.40 \mathrm{E}-02$ & $2.37 \mathrm{E}-02$ & $3.21 \mathrm{E}-02$ & $2.38 \mathrm{E}-02$ & $2.6245 \mathrm{E}+01$ & $1.0674 \mathrm{E}+03$ \\
\hline
\end{tabular}

CCCG $=3$

\begin{tabular}{|l|l|l|l|l|l|l|l|}
\hline Alpha Factor & 5 th $\%$ & Mean & Median & 95 th $\%$ & MLE & a & b \\
\hline $\mathbf{\alpha}_{1}$ & 0.9689110 & 0.9755070 & 0.9756980 & 0.9814460 & 0.9757250 & $1.5955 \mathrm{E}+03$ & $4.0060 \mathrm{E}+01$ \\
\hline $\mathbf{\alpha}_{2}$ & $1.15 \mathrm{E}-02$ & $1.62 \mathrm{E}-02$ & $1.60 \mathrm{E}-02$ & $2.17 \mathrm{E}-02$ & $1.60 \mathrm{E}-02$ & $2.6557 \mathrm{E}+01$ & $1.6090 \mathrm{E}+03$ \\
\hline $\mathbf{\alpha}_{3}$ & $4.95 \mathrm{E}-03$ & $8.26 \mathrm{E}-03$ & $8.06 \mathrm{E}-03$ & $1.22 \mathrm{E}-02$ & $8.25 \mathrm{E}-03$ & $1.3503 \mathrm{E}+01$ & $1.6221 \mathrm{E}+03$ \\
\hline
\end{tabular}

CCCG $=4$

\begin{tabular}{|l|l|l|l|l|l|l|l|}
\hline Alpha Factor & th $\%$ & Mean & Median & 95 th $\%$ & MLE & $\mathbf{a}$ & $\mathbf{b}$ \\
\hline $\mathbf{a}_{1}$ & 0.9689330 & 0.9747150 & 0.9748630 & 0.9799950 & 0.9750170 & $2.1153 \mathrm{E}+03$ & $5.4874 \mathrm{E}+01$ \\
\hline $\mathbf{a}_{2}$ & $1.13 \mathrm{E}-02$ & $1.54 \mathrm{E}-02$ & $1.53 \mathrm{E}-02$ & $2.00 \mathrm{E}-02$ & $1.52 \mathrm{E}-02$ & $3.3455 \mathrm{E}+01$ & $2.1367 \mathrm{E}+03$ \\
\hline $\mathbf{a}_{3}$ & $3.95 \mathrm{E}-03$ & $6.50 \mathrm{E}-03$ & $6.35 \mathrm{E}-03$ & $9.57 \mathrm{E}-03$ & $6.46 \mathrm{E}-03$ & $1.4109 \mathrm{E}+01$ & $2.1561 \mathrm{E}+03$ \\
\hline $\mathbf{a}_{4}$ & $1.61 \mathrm{E}-03$ & $3.37 \mathrm{E}-03$ & $3.22 \mathrm{E}-03$ & $5.64 \mathrm{E}-03$ & $3.34 \mathrm{E}-03$ & $7.3097 \mathrm{E}+00$ & $2.1629 \mathrm{E}+03$ \\
\hline
\end{tabular}

CCCG $=5$

\begin{tabular}{|l|l|l|l|l|l|l|l|}
\hline Alpha Factor & 5 th $\%$ & Mean & Median & 95 th $\%$ & MLE & $\mathbf{a}$ & $\mathbf{b}$ \\
\hline $\mathbf{a}_{1}$ & 0.9721560 & 0.9770540 & 0.9771730 & 0.9815520 & 0.9775280 & $2.6748 \mathrm{E}+03$ & $6.2818 \mathrm{E}+01$ \\
\hline $\mathbf{a}_{2}$ & $8.03 \mathrm{E}-03$ & $1.11 \mathrm{E}-02$ & $1.10 \mathrm{E}-02$ & $1.46 \mathrm{E}-02$ & $1.07 \mathrm{E}-02$ & $3.0426 \mathrm{E}+01$ & $2.7072 \mathrm{E}+03$ \\
\hline $\mathbf{a}_{3}$ & $4.24 \mathrm{E}-03$ & $6.55 \mathrm{E}-03$ & $6.43 \mathrm{E}-03$ & $9.28 \mathrm{E}-03$ & $6.43 \mathrm{E}-03$ & $1.7939 \mathrm{E}+01$ & $2.7197 \mathrm{E}+03$ \\
\hline $\mathbf{a}_{4}$ & $2.18 \mathrm{E}-03$ & $3.91 \mathrm{E}-03$ & $3.79 \mathrm{E}-03$ & $6.06 \mathrm{E}-03$ & $3.92 \mathrm{E}-03$ & $1.0711 \mathrm{E}+01$ & $2.7269 \mathrm{E}+03$ \\
\hline $\mathbf{a}_{5}$ & $4.45 \mathrm{E}-04$ & $1.37 \mathrm{E}-03$ & $1.25 \mathrm{E}-03$ & $2.70 \mathrm{E}-03$ & $1.39 \mathrm{E}-03$ & $3.7417 \mathrm{E}+00$ & $2.7339 \mathrm{E}+03$ \\
\hline
\end{tabular}

\section{CCCG $=6$}

\begin{tabular}{|l|l|l|l|l|l|l|l|}
\hline Alpha Factor & 5 th $\%$ & Mean & Median & 95 th $\%$ & MLE & $\mathbf{a}$ & $\mathbf{b}$ \\
\hline $\mathbf{a}_{1}$ & 0.9745600 & 0.9788530 & 0.9789530 & 0.9828180 & 0.9793970 & $3.2045 \mathrm{E}+03$ & $6.9231 \mathrm{E}+01$ \\
\hline $\mathbf{a}_{2}$ & $6.15 \mathrm{E}-03$ & $8.63 \mathrm{E}-03$ & $8.53 \mathrm{E}-03$ & $1.14 \mathrm{E}-02$ & $8.25 \mathrm{E}-03$ & $2.8242 \mathrm{E}+01$ & $3.2455 \mathrm{E}+03$ \\
\hline $\mathbf{a}_{3}$ & $3.83 \mathrm{E}-03$ & $5.84 \mathrm{E}-03$ & $5.74 \mathrm{E}-03$ & $8.19 \mathrm{E}-03$ & $5.70 \mathrm{E}-03$ & $1.9120 \mathrm{E}+01$ & $3.2546 \mathrm{E}+03$ \\
\hline $\mathbf{a}_{4}$ & $2.22 \mathrm{E}-03$ & $3.80 \mathrm{E}-03$ & $3.70 \mathrm{E}-03$ & $5.72 \mathrm{E}-03$ & $3.76 \mathrm{E}-03$ & $1.2439 \mathrm{E}+01$ & $3.2613 \mathrm{E}+03$ \\
\hline $\mathbf{a}_{5}$ & $1.09 \mathrm{E}-03$ & $2.27 \mathrm{E}-03$ & $2.16 \mathrm{E}-03$ & $3.78 \mathrm{E}-03$ & $2.28 \mathrm{E}-03$ & $7.4165 \mathrm{E}+00$ & $3.2663 \mathrm{E}+03$ \\
\hline $\mathbf{a}_{6}$ & $1.10 \mathrm{E}-04$ & $6.15 \mathrm{E}-04$ & $5.17 \mathrm{E}-04$ & $1.46 \mathrm{E}-03$ & $6.12 \mathrm{E}-04$ & $2.0134 \mathrm{E}+00$ & $3.2717 \mathrm{E}+03$ \\
\hline
\end{tabular}




\title{
CCF Parameter Estimations 2010, Section 2
}

\section{Section 2.2.2, Generic Rate CCF Prior Distribution: CCF-RATE}

Failure Mode :

\author{
Spurious operation open or close \\ Fail to Run (Normally running equipment) \\ Filter media allows the pass through of debris \\ Failure of Control Function Only \\ High dP across filter \\ Fail to Run $>1$ Hour (Standby equipment) \\ Fail to control flow \\ Fail to Run less than 1 Hour \\ Fail to Operate (General operation failure, rate based) \\ Loss of heat transfer capabilities in heat exchangers \\ No flow/plugged \\ 1997/01/01 \\ $2010 / 12 / 31$
}

Start Date :

Data Version :

Total Number of Independent Failure Events: 2433.50

Total Number of Common-Cause Failure Events: 99

ALPHA FACTOR DISTRIBUTIONS

CCCG $=2$

\begin{tabular}{|l|l|l|l|l|l|l|l|}
\hline Alpha Factor & th $\%$ & Mean & Median & 95 th $\%$ & MLE & a & b \\
\hline $\mathbf{a}_{1}$ & 0.9546190 & 0.9640470 & 0.9643150 & 0.9725600 & 0.9640900 & $1.1169 \mathrm{E}+03$ & $4.1654 \mathrm{E}+01$ \\
\hline $\mathbf{a}_{2}$ & $2.74 \mathrm{E}-02$ & $3.60 \mathrm{E}-02$ & $3.57 \mathrm{E}-02$ & $4.54 \mathrm{E}-02$ & $3.59 \mathrm{E}-02$ & $4.1654 \mathrm{E}+01$ & $1.1169 \mathrm{E}+03$ \\
\hline
\end{tabular}

CCCG $=3$

\begin{tabular}{|l|l|l|l|l|l|l|l|}
\hline Alpha Factor & th $\%$ & Mean & Median & 95 th $\%$ & MLE & $\mathbf{a}$ & $\mathbf{b}$ \\
\hline $\mathbf{a}_{1}$ & 0.9582460 & 0.9657470 & 0.9659280 & 0.9726330 & 0.9657760 & $1.6645 \mathrm{E}+03$ & $5.9037 \mathrm{E}+01$ \\
\hline $\mathbf{a}_{2}$ & $1.45 \mathrm{E}-02$ & $1.97 \mathrm{E}-02$ & $1.95 \mathrm{E}-02$ & $2.55 \mathrm{E}-02$ & $1.95 \mathrm{E}-02$ & $3.3908 \mathrm{E}+01$ & $1.6896 \mathrm{E}+03$ \\
\hline $\mathbf{a}_{3}$ & $1.02 \mathrm{E}-02$ & $1.46 \mathrm{E}-02$ & $1.44 \mathrm{E}-02$ & $1.96 \mathrm{E}-02$ & $1.47 \mathrm{E}-02$ & $2.5129 \mathrm{E}+01$ & $1.6984 \mathrm{E}+03$ \\
\hline
\end{tabular}

\section{CCCG $=4$}

\begin{tabular}{|l|l|l|l|l|l|l|l|}
\hline Alpha Factor & 5 th $\%$ & Mean & Median & 95 th $\%$ & MLE & a & b \\
\hline $\boldsymbol{a}_{1}$ & 0.9618670 & 0.9681350 & 0.9682740 & 0.9739420 & 0.9682810 & $2.2088 \mathrm{E}+03$ & $7.2699 \mathrm{E}+01$ \\
\hline $\mathbf{a}_{2}$ & $1.10 \mathrm{E}-02$ & $1.50 \mathrm{E}-02$ & $1.48 \mathrm{E}-02$ & $1.94 \mathrm{E}-02$ & $1.47 \mathrm{E}-02$ & $3.4148 \mathrm{E}+01$ & $2.2474 \mathrm{E}+03$ \\
\hline $\mathbf{a}_{3}$ & $6.89 \mathrm{E}-03$ & $1.01 \mathrm{E}-02$ & $9.92 \mathrm{E}-03$ & $1.37 \mathrm{E}-02$ & $1.01 \mathrm{E}-02$ & $2.2953 \mathrm{E}+01$ & $2.2585 \mathrm{E}+03$ \\
\hline $\mathbf{a}_{4}$ & $4.27 \mathrm{E}-03$ & $6.84 \mathrm{E}-03$ & $6.69 \mathrm{E}-03$ & $9.90 \mathrm{E}-03$ & $6.88 \mathrm{E}-03$ & $1.5598 \mathrm{E}+01$ & $2.2659 \mathrm{E}+03$ \\
\hline
\end{tabular}

\section{CCCG $=5$}

\begin{tabular}{|l|l|l|l|l|l|l|l|}
\hline Alpha Factor & th $\%$ & Mean & Median & 95 th $\%$ & MLE & a & b \\
\hline $\boldsymbol{a}_{1}$ & 0.9665110 & 0.9717760 & 0.9718820 & 0.9766630 & 0.9720370 & $2.7911 \mathrm{E}+03$ & $8.1064 \mathrm{E}+01$ \\
\hline $\mathbf{a}_{2}$ & $7.42 \mathrm{E}-03$ & $1.03 \mathrm{E}-02$ & $1.02 \mathrm{E}-02$ & $1.36 \mathrm{E}-02$ & $9.92 \mathrm{E}-03$ & $2.9626 \mathrm{E}+01$ & $2.8425 \mathrm{E}+03$ \\
\hline $\boldsymbol{\alpha}_{3}$ & $5.93 \mathrm{E}-03$ & $8.55 \mathrm{E}-03$ & $8.44 \mathrm{E}-03$ & $1.16 \mathrm{E}-02$ & $8.51 \mathrm{E}-03$ & $2.4566 \mathrm{E}+01$ & $2.8476 \mathrm{E}+03$ \\
\hline $\boldsymbol{a}_{4}$ & $3.54 \mathrm{E}-03$ & $5.62 \mathrm{E}-03$ & $5.50 \mathrm{E}-03$ & $8.09 \mathrm{E}-03$ & $5.69 \mathrm{E}-03$ & $1.6139 \mathrm{E}+01$ & $2.8560 \mathrm{E}+03$ \\
\hline $\mathbf{a}_{5}$ & $2.08 \mathrm{E}-03$ & $3.74 \mathrm{E}-03$ & $3.62 \mathrm{E}-03$ & $5.78 \mathrm{E}-03$ & $3.85 \mathrm{E}-03$ & $1.0733 \mathrm{E}+01$ & $2.8614 \mathrm{E}+03$ \\
\hline
\end{tabular}

\section{CCCG $=6$}

\begin{tabular}{|l|l|l|l|l|l|l|l|}
\hline Alpha Factor & 5 th $\%$ & Mean & Median & 95 th $\%$ & MLE & a & $\mathbf{b}$ \\
\hline $\mathbf{a}_{1}$ & 0.9701220 & 0.9746820 & 0.9747770 & 0.9789330 & 0.9750480 & $3.3448 \mathrm{E}+03$ & $8.6882 \mathrm{E}+01$ \\
\hline $\boldsymbol{a}_{2}$ & $5.07 \mathrm{E}-03$ & $7.29 \mathrm{E}-03$ & $7.19 \mathrm{E}-03$ & $9.83 \mathrm{E}-03$ & $6.88 \mathrm{E}-03$ & $2.5014 \mathrm{E}+01$ & $3.4067 \mathrm{E}+03$ \\
\hline $\boldsymbol{a}_{3}$ & $4.91 \mathrm{E}-03$ & $7.10 \mathrm{E}-03$ & $7.00 \mathrm{E}-03$ & $9.60 \mathrm{E}-03$ & $7.01 \mathrm{E}-03$ & $2.4348 \mathrm{E}+01$ & $3.4073 \mathrm{E}+03$ \\
\hline $\mathbf{a}_{4}$ & $3.55 \mathrm{E}-03$ & $5.44 \mathrm{E}-03$ & $5.34 \mathrm{E}-03$ & $7.65 \mathrm{E}-03$ & $5.46 \mathrm{E}-03$ & $1.8666 \mathrm{E}+01$ & $3.4130 \mathrm{E}+03$ \\
\hline $\mathbf{a}_{5}$ & $1.67 \mathrm{E}-03$ & $3.03 \mathrm{E}-03$ & $2.94 \mathrm{E}-03$ & $4.72 \mathrm{E}-03$ & $3.08 \mathrm{E}-03$ & $1.0408 \mathrm{E}+01$ & $3.4213 \mathrm{E}+03$ \\
\hline $\mathbf{a}_{6}$ & $1.25 \mathrm{E}-03$ & $2.46 \mathrm{E}-03$ & $2.37 \mathrm{E}-03$ & $4.00 \mathrm{E}-03$ & $2.53 \mathrm{E}-03$ & $8.4458 \mathrm{E}+00$ & $3.4232 \mathrm{E}+03$ \\
\hline
\end{tabular}


CCF Parameter Estimations 2012, Section 2, No Data (Prior Only)

\section{Section 2.2.3, CCF Prior Distribution, Data Version 2007/12/31}

Data Version :

$2007 / 12 / 31$

Total Number of Independent Failure Events: 0

Total Number of Common-Cause Failure Events: 0

\section{ALPHA FACTOR DISTRIBUTIONS}

$C C C G=2$

\begin{tabular}{|l|l|l|l|l|l|l|l|}
\hline Alpha Factor & 5 th $\%$ & Mean & Median & 95 th $\%$ & MLE & a & b \\
\hline $\mathbf{\alpha}_{1}$ & 0.8993200 & 0.9742690 & 0.9887700 & 0.9999290 & -- & $1.7418 \mathrm{E}+01$ & $4.6002 \mathrm{E}-01$ \\
\hline $\mathbf{\alpha}_{2}$ & $6.65 \mathrm{E}-05$ & $2.57 \mathrm{E}-02$ & $1.12 \mathrm{E}-02$ & $1.00 \mathrm{E}-01$ & -- & $4.6002 \mathrm{E}-01$ & $1.7418 \mathrm{E}+01$ \\
\hline
\end{tabular}

$\mathrm{CCCG}=3$

\begin{tabular}{|l|l|l|l|l|l|l|l|}
\hline Alpha Factor & 5 th $\%$ & Mean & Median & 95 th $\%$ & MLE & a & b \\
\hline $\mathbf{a}_{1}$ & 0.9306240 & 0.9755060 & 0.9819700 & 0.9982830 & -- & $4.5105 \mathrm{E}+01$ & $1.1325 \mathrm{E}+00$ \\
\hline $\mathbf{a}_{2}$ & $6.61 \mathrm{E}-04$ & $1.87 \mathrm{E}-02$ & $1.23 \mathrm{E}-02$ & $5.84 \mathrm{E}-02$ & -- & $8.6476 \mathrm{E}-01$ & $4.5372 \mathrm{E}+01$ \\
\hline $\mathbf{a}_{3}$ & $2.07 \mathrm{E}-07$ & $5.79 \mathrm{E}-03$ & $1.17 \mathrm{E}-03$ & $2.74 \mathrm{E}-02$ & -- & $2.6776 \mathrm{E}-01$ & $4.5969 \mathrm{E}+01$ \\
\hline
\end{tabular}

$\mathrm{CCCG}=4$

\begin{tabular}{|l|l|l|l|l|l|l|l|}
\hline Alpha Factor & 5 th\% & Mean & Median & 95 th\% & MLE & $\mathbf{a}$ & b \\
\hline $\mathbf{a}_{1}$ & 0.9380870 & 0.9740820 & 0.9782970 & 0.9956540 & -- & $7.0868 \mathrm{E}+01$ & $1.8856 \mathrm{E}+00$ \\
\hline $\mathbf{a}_{2}$ & $1.43 \mathrm{E}-03$ & $1.70 \mathrm{E}-02$ & $1.28 \mathrm{E}-02$ & $4.69 \mathrm{E}-02$ & -- & $1.2400 \mathrm{E}+00$ & $7.1513 \mathrm{E}+01$ \\
\hline $\mathbf{a}_{3}$ & $9.66 \mathrm{E}-06$ & $5.89 \mathrm{E}-03$ & $2.32 \mathrm{E}-03$ & $2.38 \mathrm{E}-02$ & -- & $4.2870 \mathrm{E}-01$ & $7.2324 \mathrm{E}+01$ \\
\hline $\mathbf{a}_{4}$ & $9.21 \mathrm{E}-09$ & $2.98 \mathrm{E}-03$ & $3.83 \mathrm{E}-04$ & $1.50 \mathrm{E}-02$ & -- & $2.1695 \mathrm{E}-01$ & $7.2536 \mathrm{E}+01$ \\
\hline
\end{tabular}

$\mathrm{CCCG}=5$

\begin{tabular}{|l|l|l|l|l|l|l|l|}
\hline Alpha Factor & 5 th\% & Mean & Median & 95 th\% & MLE & $\mathbf{a}$ & $\mathbf{b}$ \\
\hline $\mathbf{a}_{1}$ & 0.9521790 & 0.9760740 & 0.9782400 & 0.9925770 & -- & $1.4106 \mathrm{E}+02$ & $3.4576 \mathrm{E}+00$ \\
\hline $\mathbf{a}_{2}$ & $2.59 \mathrm{E}-03$ & $1.41 \mathrm{E}-02$ & $1.19 \mathrm{E}-02$ & $3.30 \mathrm{E}-02$ & -- & $2.0400 \mathrm{E}+00$ & $1.4247 \mathrm{E}+02$ \\
\hline $\mathbf{a}_{3}$ & $3.01 \mathrm{E}-04$ & $6.59 \mathrm{E}-03$ & $4.50 \mathrm{E}-03$ & $2.00 \mathrm{E}-02$ & -- & $9.5369 \mathrm{E}-01$ & $1.4356 \mathrm{E}+02$ \\
\hline $\mathbf{a}_{4}$ & $2.21 \mathrm{E}-06$ & $2.67 \mathrm{E}-03$ & $9.37 \mathrm{E}-04$ & $1.12 \mathrm{E}-02$ & -- & $3.8684 \mathrm{E}-01$ & $1.4413 \mathrm{E}+02$ \\
\hline $\mathbf{a}_{5}$ & $5.61 \mathrm{E}-20$ & $5.33 \mathrm{E}-04$ & $5.18 \mathrm{E}-07$ & $3.10 \mathrm{E}-03$ & -- & $7.7129 \mathrm{E}-02$ & $1.4444 \mathrm{E}+02$ \\
\hline
\end{tabular}

$\mathrm{CCCG}=6$

\begin{tabular}{|l|l|l|l|l|l|l|l|}
\hline Alpha Factor & 5th\% & Mean & Median & 95 th\% & MLE & a & b \\
\hline $\mathbf{a}_{1}$ & 0.9553700 & 0.9762820 & 0.9779970 & 0.9913440 & -- & $1.7893 \mathrm{E}+02$ & $4.3470 \mathrm{E}+00$ \\
\hline $\mathbf{a}_{2}$ & $2.60 \mathrm{E}-03$ & $1.24 \mathrm{E}-02$ & $1.07 \mathrm{E}-02$ & $2.81 \mathrm{E}-02$ & -- & $2.2804 \mathrm{E}+00$ & $1.8099 \mathrm{E}+02$ \\
\hline $\mathbf{a}_{3}$ & $4.16 \mathrm{E}-04$ & $6.13 \mathrm{E}-03$ & $4.45 \mathrm{E}-03$ & $1.75 \mathrm{E}-02$ & -- & $1.1245 \mathrm{E}+00$ & $1.8215 \mathrm{E}+02$ \\
\hline $\mathbf{a}_{4}$ & $3.82 \mathrm{E}-05$ & $3.40 \mathrm{E}-03$ & $1.85 \mathrm{E}-03$ & $1.20 \mathrm{E}-02$ & -- & $6.2471 \mathrm{E}-01$ & $1.8265 \mathrm{E}+02$ \\
\hline $\mathbf{a}_{5}$ & $1.60 \mathrm{E}-08$ & $1.32 \mathrm{E}-03$ & $2.18 \mathrm{E}-04$ & $6.46 \mathrm{E}-03$ & -- & $2.4272 \mathrm{E}-01$ & $1.8303 \mathrm{E}+02$ \\
\hline $\mathbf{a}_{6}$ & $1.26 \mathrm{E}-20$ & $4.07 \mathrm{E}-04$ & $3.05 \mathrm{E}-07$ & $2.36 \mathrm{E}-03$ & -- & $7.4722 \mathrm{E}-02$ & $1.8320 \mathrm{E}+02$ \\
\hline
\end{tabular}

\section{$\mathrm{CCCG}=7$}

\begin{tabular}{|l|l|l|l|l|l|l|l|}
\hline Alpha Factor & 5th\% & Mean & Median & 95 th\% & MLE & a & b \\
\hline $\mathbf{a}_{1}$ & 0.9603690 & 0.9769760 & 0.9781320 & 0.9896440 & -- & $2.6720 \mathrm{E}+02$ & $6.2971 \mathrm{E}+00$ \\
\hline $\mathbf{\alpha}_{2}$ & $3.14 \mathrm{E}-03$ & $1.12 \mathrm{E}-02$ & $1.00 \mathrm{E}-02$ & $2.33 \mathrm{E}-02$ & -- & $3.0721 \mathrm{E}+00$ & $2.7042 \mathrm{E}+02$ \\
\hline $\mathbf{a}_{3}$ & $6.66 \mathrm{E}-04$ & $5.55 \mathrm{E}-03$ & $4.40 \mathrm{E}-03$ & $1.43 \mathrm{E}-02$ & -- & $1.5182 \mathrm{E}+00$ & $2.7197 \mathrm{E}+02$ \\
\hline $\mathbf{a}_{4}$ & $1.58 \mathrm{E}-04$ & $3.48 \mathrm{E}-03$ & $2.37 \mathrm{E}-03$ & $1.06 \mathrm{E}-02$ & -- & $9.5310 \mathrm{E}-01$ & $2.7254 \mathrm{E}+02$ \\
\hline $\mathbf{a}_{5}$ & $1.00 \mathrm{E}-05$ & $1.93 \mathrm{E}-03$ & $9.22 \mathrm{E}-04$ & $7.26 \mathrm{E}-03$ & -- & $5.2795 \mathrm{E}-01$ & $2.7296 \mathrm{E}+02$ \\
\hline $\mathbf{a}_{6}$ & $4.58 \mathrm{E}-10$ & $7.08 \mathrm{E}-04$ & $6.75 \mathrm{E}-05$ & $3.68 \mathrm{E}-03$ & -- & $1.9373 \mathrm{E}-01$ & $2.7330 \mathrm{E}+02$ \\
\hline $\mathbf{\alpha} 7$ & $5.03 \mathrm{E}-44$ & $1.17 \mathrm{E}-04$ & $8.41 \mathrm{E}-13$ & $4.81 \mathrm{E}-04$ & -- & $3.2027 \mathrm{E}-02$ & $2.7346 \mathrm{E}+02$ \\
\hline
\end{tabular}

\section{$\mathrm{CCCG}=8$}

\begin{tabular}{|l|l|l|l|l|l|l|l|}
\hline Alpha Factor & 5th\% & Mean & Median & 95 th\% & MLE & a & $\mathbf{b}$ \\
\hline $\mathbf{a}_{1}$ & 0.9622170 & 0.9773660 & 0.9783580 & 0.9891370 & -- & $3.1221 \mathrm{E}+02$ & $7.2302 \mathrm{E}+00$ \\
\hline $\mathbf{a}_{2}$ & $3.13 \mathrm{E}-03$ & $1.04 \mathrm{E}-02$ & $9.45 \mathrm{E}-03$ & $2.12 \mathrm{E}-02$ & -- & $3.3414 \mathrm{E}+00$ & $3.1609 \mathrm{E}+02$ \\
\hline $\mathbf{a}_{3}$ & $6.67 \mathrm{E}-04$ & $5.04 \mathrm{E}-03$ & $4.06 \mathrm{E}-03$ & $1.28 \mathrm{E}-02$ & -- & $1.6130 \mathrm{E}+00$ & $3.1782 \mathrm{E}+02$ \\
\hline $\mathbf{a}_{4}$ & $1.86 \mathrm{E}-04$ & $3.26 \mathrm{E}-03$ & $2.30 \mathrm{E}-03$ & $9.62 \mathrm{E}-03$ & -- & $1.0438 \mathrm{E}+00$ & $3.1839 \mathrm{E}+02$ \\
\hline $\mathbf{a} 5$ & $3.88 \mathrm{E}-05$ & $2.20 \mathrm{E}-03$ & $1.28 \mathrm{E}-03$ & $7.47 \mathrm{E}-03$ & -- & $7.0280 \mathrm{E}-01$ & $3.1873 \mathrm{E}+02$ \\
\hline $\mathbf{a}_{6}$ & $5.77 \mathrm{E}-07$ & $1.13 \mathrm{E}-03$ & $3.63 \mathrm{E}-04$ & $4.86 \mathrm{E}-03$ & -- & $3.6184 \mathrm{E}-01$ & $3.1907 \mathrm{E}+02$ \\
\hline $\mathbf{a}_{7}$ & $1.19 \mathrm{E}-13$ & $3.98 \mathrm{E}-04$ & $8.44 \mathrm{E}-06$ & $2.25 \mathrm{E}-03$ & -- & $1.2739 \mathrm{E}-01$ & $3.1931 \mathrm{E}+02$ \\
\hline $\mathbf{a}_{8}$ & $5.47 \mathrm{E}-36$ & $1.25 \mathrm{E}-04$ & $5.43 \mathrm{E}-11$ & $6.01 \mathrm{E}-04$ & -- & $4.0005 \mathrm{E}-02$ & $3.1940 \mathrm{E}+02$ \\
\hline
\end{tabular}




\section{CCF Parameter Estimations 2012, Section 2}

\section{Section 2.2.1, Generic Demand CCF Prior Distribution: CCF-DEM}

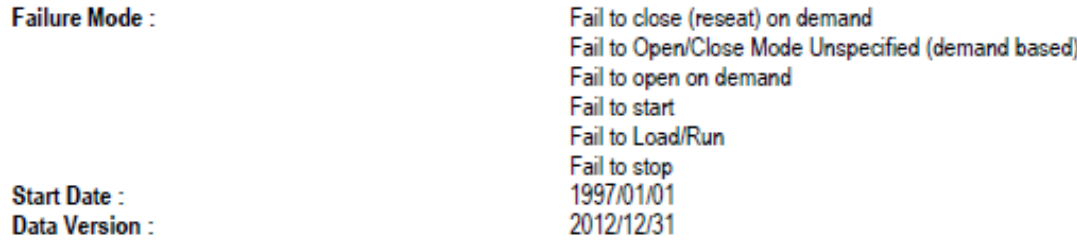

Total Number of Independent Failure Events: 3104.30

Total Number of Common-Cause Failure Events: 102

\section{ALPHA FACTOR DISTRIBUTIONS}

$C C C G=2$

Alpha Factor 5th\%

$\mathbf{a}_{2}$

$\mathrm{CCCG}=3$

Alpha Factor 5th\%

$\mathrm{CCCG}=4$

Alpha Factor 5 th\%

a1 $\quad 0.9698830$

a2 $\quad 1.18 \mathrm{E}-02$

a3 $3.62 \mathrm{E}-03$

a4 $\quad 1.40 \mathrm{E}-03$

$\mathrm{CCCG}=5$

Alpha Factor 5 th\%

$a_{1} \quad 0.9730170$

$\mathrm{a}_{2} \quad 8.24 \mathrm{E}-03$

$\alpha_{3} \quad 4.22 \mathrm{E}-03$

$\boldsymbol{a}_{4} \quad 1.96 \mathrm{E}-03$

a5 $3.83 \mathrm{E}-04$

$\mathrm{CCCG}=6$

Alpha Factor 5 th $\%$

$\begin{array}{ll}\mathbf{a}_{1} & 0.9753380 \\ \mathbf{a}_{2} & 6.25 \mathrm{E}-03 \\ \mathbf{d}_{3} & 3.92 \mathrm{E}-03 \\ \mathbf{a}_{4} & 2.10 \mathrm{E}-03 \\ \mathbf{a}_{5} & 9.73 \mathrm{E}-04 \\ \mathbf{d}_{5} & 9.37 \mathrm{E}-05\end{array}$

$\mathbf{a}_{6} \quad 9.37 \mathrm{E}-05$

$\mathrm{CCCG}=7$

Alpha Factor 5th\%

ai $\quad 0.9771660$

a2 5.19E-03

a3 $3.24 \mathrm{E}-03$

$a_{4} \quad 2.15 \mathrm{E}-03$

a5 1.25E-03

a6 4.39E-04

a7 $1.37 \mathrm{E}-05$

$\mathrm{CCCG}=8$

Alpha Factor 5 th\%

0.9786350

$\mathrm{a}_{2} \quad 4.54 \mathrm{E}-03$

$a_{3} 2.69 E-03$

a4 1.93E-03

1.34E-03

$1.34 \mathrm{E}-03$
$7.16 \mathrm{E}-04$

$1.75 \mathrm{E}-04$

$1.12 E-06$

$\begin{array}{lll}\text { Mean } & \text { Median } & 95 \text { th } \% \\ 0.9781220 & 0.9783890 & 0.9845880 \\ 2.19 E-02 & 2.16 \text { E-02 } & 2.92 \mathrm{E}-02\end{array}$

MLE

$0.9782910 \quad 1.1730 \mathrm{E}+03 \quad 26237 \mathrm{E}+01$ 2. $19 E-02$

Mean

0.9765760

$1.62 \mathrm{E}-02$

Median 0.9767500

1.60E-02

$7.08 \mathrm{E}-03$

95 th\%

0.9821350

2. $13 \mathrm{E}-02$

$1.08 \mathrm{E}-02$

MLE 0.9767940 $2.8978 \mathrm{E}+01 \quad 1.7640 \mathrm{E}+03$

$7.24 \mathrm{E}-03 \quad 1.3021 \mathrm{E}+01 \quad 1.7800 \mathrm{E}+03$

0.9753330

1.57E-02

$5.96 \mathrm{E}-03$

Median

0.9754690

$1.56 \mathrm{E}-02$

$5.82 \mathrm{E}-03$

2.83E- 03

95th\%

0.9803240

2.02E-02

8.77E-03

5.01E-03

MLE

$0.9756210 \quad 2.3202 E+03 \quad 5.8679 E+01$ $1.55 \mathrm{E}-02 \quad 3.7438 \mathrm{E}+01 \quad 2.3414 \mathrm{E}+03$ 5.91E-03 $\quad 1.4181 \mathrm{E}+01 \quad 2.3647 \mathrm{E}+03$ $2.93 \mathrm{E}-03 \quad 7.0597 \mathrm{E}+00 \quad 2.3718 \mathrm{E}+03$

Mean

0.9776340

$1.12 \mathrm{E}-02$

$6.42 \mathrm{E}-03$

$3.53 E-03$

$1.21 \mathrm{E}-03$

Median

0.9777440

1.11E-02

$6.31 \mathrm{E}-03$

$3.42 \mathrm{E}-03$

1.10E-03

Mean

0.9793890

8.63E-03

5.84E-03

$3.56 \mathrm{E}-03$

2.03E-03

5.44E-04

Median

0.9794810

8.54E-03

$5.75 \mathrm{E}-03$

$3.47 \mathrm{E}-03$

1.94E-03

4.55E-04

Mean

0.9807650

7.19E-03

4.85E-03

3. $49 \mathrm{E}-03$

2.31E-03

1.14E-03

2.46E-04

Median

0.9808440

7.11E-03

4.77E-03

3. $42 \mathrm{E}-03$

2.24E-03

$1.06 \mathrm{E}-03$

1.73E-04

Mean

0.9819010

6.29E-03

4.07E-03

3.12E-03

2.36E-03

$1.50 \mathrm{E}-03$

6.33E-04

1.23E-04
Median

0.9819650

6.23E-03

4.00E-03

3.06E-03

$2.29 \mathrm{E}-03$

1.43E-03

$5.66 \mathrm{E}-04$

6.45E-05
0.9818860

$1.45 \mathrm{E}-02$

$8.99 \mathrm{E}-03$

$5.48 \mathrm{E}-03$

2.40E-03

95th\%

0.9831360

1.13E-02

8.08E-03

5.34E-03

3.41E-03

1.30E-03

95 th $\%$

0.9841110

9.45E-03

6.73E-03

$5.11 \mathrm{E}-03$

3.65E-03

2.11E-03

7.27E-04

95 th $\%$

0.9849440

8.28E-03

$5.68 \mathrm{E}-03$

4.55E-03

3.61E-03

2.52E-03

1.32E-03

4.45E-04
MLE a

$0.9780860 \quad 2.9304 E+03 \quad 6.7040 E+01$ $1.09 E-02 \quad 3.3592 E+01 \quad 2.9638 E+03$ 6.30E-03 $\quad 1.9244 E+01 \quad 2.9782 E+03$

$3.52 \mathrm{E}-03 \quad 1.0587 \mathrm{E}+01 \quad 2.9869 \mathrm{E}+03$

$\begin{array}{lll}1.22 \mathrm{E}-03 & 3.6167 \mathrm{E}+00 \quad 2.9938 \mathrm{E}+03\end{array}$

MLE

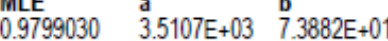
$8.28 \mathrm{E}-03 \quad 3.0929 \mathrm{E}+01 \quad 3.5537 \mathrm{E}+03$ $5.72 \mathrm{E}-03 \quad 2.0938 \mathrm{E}+01 \quad 3.5636 \mathrm{E}+03$ $3.52 \mathrm{E}-03 \quad 1.2773 \mathrm{E}+01 \quad 3.5718 \mathrm{E}+03$ $2.04 \mathrm{E}-03 \quad 7.2915 \mathrm{E}+00 \quad 3.5773 \mathrm{E}+03$ 5.39E-04 1.9509E+00 3.5826E+03

$\begin{array}{lll}\text { MLE } & \text { a } & \text { b } \\ 0.9814550 & 4.1375 E+03 & 8.1145 E+01\end{array}$ $6.76 \mathrm{E}-03 \quad 3.0330 \mathrm{E}+01 \quad 4.1883 \mathrm{E}+03$ $4.68 \mathrm{E}-03 \quad 2.0467 \mathrm{E}+01 \quad 4.1982 \mathrm{E}+03$ $3.41 \mathrm{E}-03 \quad 1.4740 \mathrm{E}+01 \quad 4.2039 \mathrm{E}+03$ $2.29 \mathrm{E}-03 \quad 9.7630 \mathrm{E}+00 \quad 4.2089 \mathrm{E}+03$ $1.15 \mathrm{E}-03 \quad 4.8083 \mathrm{E}+00 \quad 4.2138 \mathrm{E}+03$ $2.50 \mathrm{E}-04 \quad 1.0362 \mathrm{E}+00 \quad 4.2176 \mathrm{E}+03$

MLE 0.9826340 $5.88 \mathrm{E}-03$ 3.89E-03 $3.03 \mathrm{E}-03$ $2.32 \mathrm{E}-03$ $1.50 \mathrm{E}-03$

$6.38 \mathrm{E}-04$

1.22E-04 a b $8.7210 E+01$ $3.0325 \mathrm{E}+01 \quad 4.7881 \mathrm{E}+03$ $1.9601 \mathrm{E}+01 \quad 4.7988 \mathrm{E}+03$ $1.5052 \mathrm{E}+01 \quad 4.8034 \mathrm{E}+03$ $1.1349 E+01 \quad 4.8071 E+03$ $7.2366 \mathrm{E}+00 \quad 4.8112 \mathrm{E}+03$ $3.0513 \mathrm{E}+00 \quad 4.8154 \mathrm{E}+03$ $5.9462 \mathrm{E}-01 \quad 4.8178 \mathrm{E}+03$ 


\section{CCF Parameter Estimations 2012, Section 2}

\section{Section 2.2.2, Generic Rate CCF Prior Distribution: CCF-RATE}

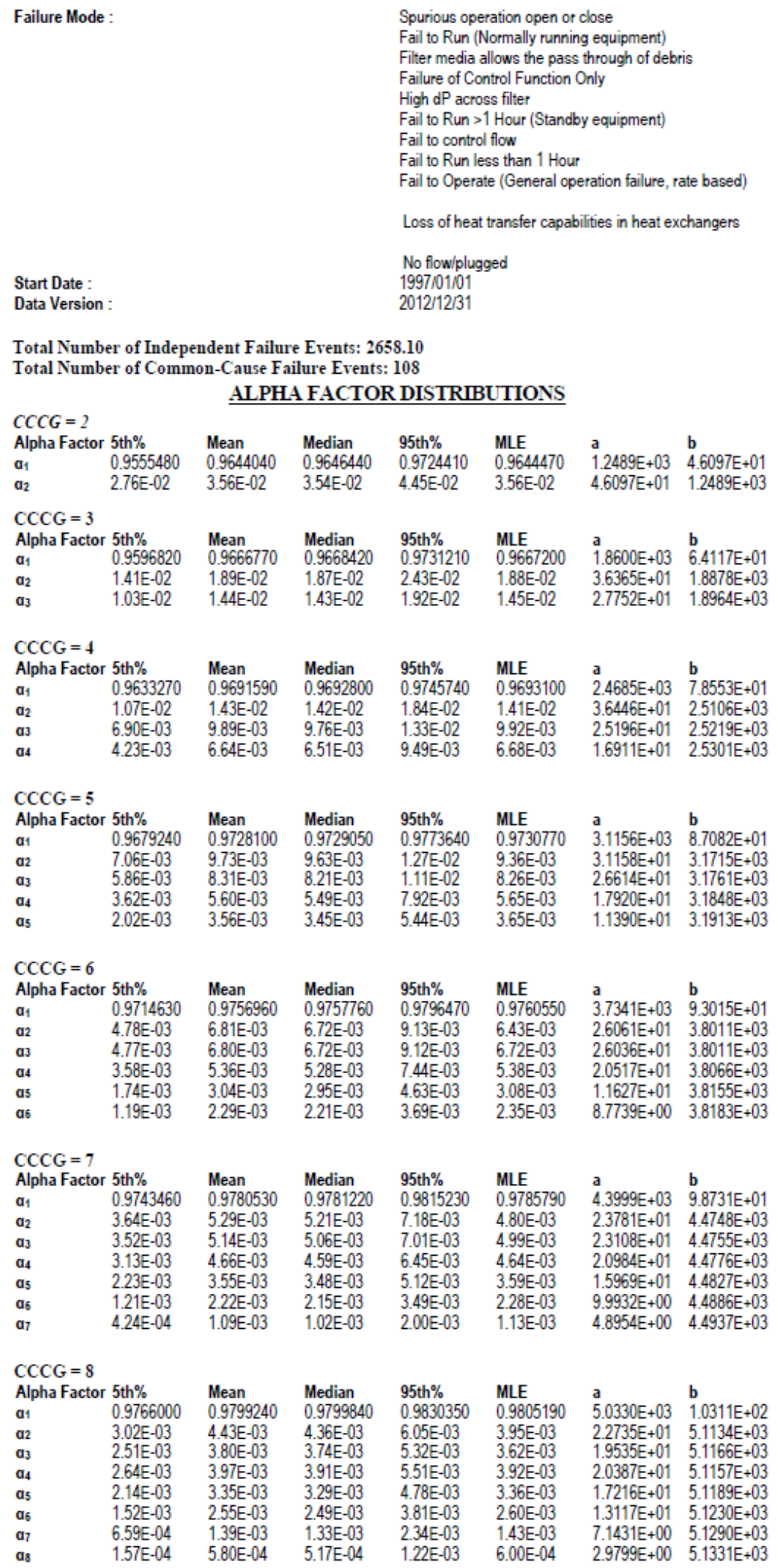


CCF Parameter Estimations 2015, Section 3, No Data (Prior Only)

\section{Section 3.1.3, CCF Prior Distribution, Data Version 2007/12/31}

Data Version :

$2007 / 12 / 31$

Total Number of Independent Failure Events: 0

Total Number of Common-Cause Failure Events: 0

\section{ALPHA FACTOR DISTRIBUTIONS}

$C C C G=2$

\begin{tabular}{|l|l|l|l|l|l|l|l|}
\hline Alpha Factor & 5 th $\%$ & Mean & Median & 95 th $\%$ & MLE & $\mathbf{a}$ & $\mathbf{b}$ \\
\hline $\mathbf{a}_{1}$ & 0.8993200 & 0.9742690 & 0.9887700 & 0.9999290 & -- & $1.7418 \mathrm{E}+01$ & $4.6002 \mathrm{E}-01$ \\
\hline $\boldsymbol{\alpha}_{2}$ & $6.65 \mathrm{E}-05$ & $2.57 \mathrm{E}-02$ & $1.12 \mathrm{E}-02$ & $1.00 \mathrm{E}-01$ & -- & $4.6002 \mathrm{E}-01$ & $1.7418 \mathrm{E}+01$ \\
\hline
\end{tabular}

$\mathrm{CCCG}=3$

\begin{tabular}{|l|l|l|l|l|l|l|l|}
\hline Alpha Factor & 5 th $\%$ & Mean & Median & 95 th $\%$ & MLE & a & b \\
\hline $\mathbf{a}_{1}$ & 0.9306240 & 0.9755060 & 0.9819700 & 0.9982830 & -- & $4.5105 \mathrm{E}+01$ & $1.1325 \mathrm{E}+00$ \\
\hline $\boldsymbol{\alpha}_{2}$ & $6.61 \mathrm{E}-04$ & $1.87 \mathrm{E}-02$ & $1.23 \mathrm{E}-02$ & $5.84 \mathrm{E}-02$ & -- & $8.6476 \mathrm{E}-01$ & $4.5372 \mathrm{E}+01$ \\
\hline $\boldsymbol{\alpha}_{3}$ & $2.07 \mathrm{E}-07$ & $5.79 \mathrm{E}-03$ & $1.17 \mathrm{E}-03$ & $2.74 \mathrm{E}-02$ & -- & $2.6776 \mathrm{E}-01$ & $4.5969 \mathrm{E}+01$ \\
\hline
\end{tabular}

\section{$\mathrm{CCCG}=\mathbf{4}$}

\begin{tabular}{|l|l|l|l|l|l|l|l|}
\hline Alpha Factor & 5 th $\%$ & Mean & Median & 95 th $\%$ & MLE & a & b \\
\hline $\boldsymbol{a}_{1}$ & 0.9380870 & 0.9740820 & 0.9782970 & 0.9956540 & -- & $7.0868 \mathrm{E}+01$ & $1.8856 \mathrm{E}+00$ \\
\hline $\boldsymbol{\alpha}_{2}$ & $1.43 \mathrm{E}-03$ & $1.70 \mathrm{E}-02$ & $1.28 \mathrm{E}-02$ & $4.69 \mathrm{E}-02$ & --- & $1.2400 \mathrm{E}+00$ & $7.1513 \mathrm{E}+01$ \\
\hline $\boldsymbol{a}_{3}$ & $9.66 \mathrm{E}-06$ & $5.89 \mathrm{E}-03$ & $2.32 \mathrm{E}-03$ & $2.38 \mathrm{E}-02$ & -- & $4.2870 \mathrm{E}-01$ & $7.2324 \mathrm{E}+01$ \\
\hline $\boldsymbol{a}_{4}$ & $9.21 \mathrm{E}-09$ & $2.98 \mathrm{E}-03$ & $3.83 \mathrm{E}-04$ & $1.50 \mathrm{E}-02$ & -- & $2.1695 \mathrm{E}-01$ & $7.2536 \mathrm{E}+01$ \\
\hline
\end{tabular}

CCCG = 5
\begin{tabular}{|l|l|l|l|l|l|l|l|}
\hline Alpha Factor & 5 th $\%$ & Mean & Median & 95 th $\%$ & MLE & a & b \\
\hline $\boldsymbol{\alpha}_{1}$ & 0.9521790 & 0.9760740 & 0.9782400 & 0.9925770 & -- & $1.4106 \mathrm{E}+02$ & $3.4576 \mathrm{E}+00$ \\
\hline $\boldsymbol{\alpha}_{2}$ & $2.59 \mathrm{E}-03$ & $1.41 \mathrm{E}-02$ & $1.19 \mathrm{E}-02$ & $3.30 \mathrm{E}-02$ & -- & $2.0400 \mathrm{E}+00$ & $1.4247 \mathrm{E}+02$ \\
\hline $\boldsymbol{\alpha}_{3}$ & $3.01 \mathrm{E}-04$ & $6.59 \mathrm{E}-03$ & $4.50 \mathrm{E}-03$ & $2.00 \mathrm{E}-02$ & -- & $9.5369 \mathrm{E}-01$ & $1.4356 \mathrm{E}+02$ \\
\hline $\boldsymbol{\alpha}_{4}$ & $2.21 \mathrm{E}-06$ & $2.67 \mathrm{E}-03$ & $9.37 \mathrm{E}-04$ & $1.12 \mathrm{E}-02$ & -- & $3.8684 \mathrm{E}-01$ & $1.4413 \mathrm{E}+02$ \\
\hline $\boldsymbol{\alpha}_{5}$ & $5.61 \mathrm{E}-20$ & $5.33 \mathrm{E}-04$ & $5.18 \mathrm{E}-07$ & $3.10 \mathrm{E}-03$ & --- & $7.7129 \mathrm{E}-02$ & $1.4444 \mathrm{E}+02$ \\
\hline
\end{tabular}

CCCG = 6
\begin{tabular}{|l|l|l|l|l|l|l|l|}
\hline Alpha Factor & 5 th $\%$ & Mean & Median & 95 th $\%$ & MLE & a & b \\
\hline $\boldsymbol{\alpha}_{1}$ & 0.9553700 & 0.9762820 & 0.9779970 & 0.9913440 & -- & $1.7893 \mathrm{E}+02$ & $4.3470 \mathrm{E}+00$ \\
\hline $\boldsymbol{\alpha}_{2}$ & $2.60 \mathrm{E}-03$ & $1.24 \mathrm{E}-02$ & $1.07 \mathrm{E}-02$ & $2.81 \mathrm{E}-02$ & -- & $2.2804 \mathrm{E}+00$ & $1.8099 \mathrm{E}+02$ \\
\hline $\boldsymbol{\alpha}_{3}$ & $4.16 \mathrm{E}-04$ & $6.13 \mathrm{E}-03$ & $4.45 \mathrm{E}-03$ & $1.75 \mathrm{E}-02$ & -- & $1.1245 \mathrm{E}+00$ & $1.8215 \mathrm{E}+02$ \\
\hline $\boldsymbol{\alpha}_{4}$ & $3.82 \mathrm{E}-05$ & $3.40 \mathrm{E}-03$ & $1.85 \mathrm{E}-03$ & $1.20 \mathrm{E}-02$ & -- & $6.2471 \mathrm{E}-01$ & $1.8265 \mathrm{E}+02$ \\
\hline $\boldsymbol{a}_{5}$ & $1.60 \mathrm{E}-08$ & $1.32 \mathrm{E}-03$ & $2.18 \mathrm{E}-04$ & $6.46 \mathrm{E}-03$ & -- & $2.4272 \mathrm{E}-01$ & $1.8303 \mathrm{E}+02$ \\
\hline $\boldsymbol{a}_{6}$ & $1.26 \mathrm{E}-20$ & $4.07 \mathrm{E}-04$ & $3.05 \mathrm{E}-07$ & $2.36 \mathrm{E}-03$ & --- & $7.4722 \mathrm{E}-02$ & $1.8320 \mathrm{E}+02$ \\
\hline
\end{tabular}

$\mathrm{CCCG}=7$

\begin{tabular}{|l|l|l|l|l|l|l|l|}
\hline Alpha Factor & 5 th $\%$ & Mean & Median & 95 th $\%$ & MLE & a & b \\
\hline $\boldsymbol{\alpha}_{1}$ & 0.9603690 & 0.9769760 & 0.9781320 & 0.9896440 & -- & $2.6720 \mathrm{E}+02$ & $6.2971 \mathrm{E}+00$ \\
\hline $\boldsymbol{\alpha}_{2}$ & $3.14 \mathrm{E}-03$ & $1.12 \mathrm{E}-02$ & $1.00 \mathrm{E}-02$ & $2.33 \mathrm{E}-02$ & -- & $3.0721 \mathrm{E}+00$ & $2.7042 \mathrm{E}+02$ \\
\hline $\boldsymbol{\alpha}_{3}$ & $6.66 \mathrm{E}-04$ & $5.55 \mathrm{E}-03$ & $4.40 \mathrm{E}-03$ & $1.43 \mathrm{E}-02$ & -- & $1.5182 \mathrm{E}+00$ & $2.7197 \mathrm{E}+02$ \\
\hline $\boldsymbol{\alpha}_{4}$ & $1.58 \mathrm{E}-04$ & $3.48 \mathrm{E}-03$ & $2.37 \mathrm{E}-03$ & $1.06 \mathrm{E}-02$ & -- & $9.5310 \mathrm{E}-01$ & $2.7254 \mathrm{E}+02$ \\
\hline $\boldsymbol{\alpha}_{5}$ & $1.00 \mathrm{E}-05$ & $1.93 \mathrm{E}-03$ & $9.22 \mathrm{E}-04$ & $7.26 \mathrm{E}-03$ & -- & $5.2795 \mathrm{E}-01$ & $2.7296 \mathrm{E}+02$ \\
\hline $\boldsymbol{\alpha}_{6}$ & $4.58 \mathrm{E}-10$ & $7.08 \mathrm{E}-04$ & $6.75 \mathrm{E}-05$ & $3.68 \mathrm{E}-03$ & -- & $1.9373 \mathrm{E}-01$ & $2.7330 \mathrm{E}+02$ \\
\hline $\boldsymbol{\alpha}_{7}$ & $5.03 \mathrm{E}-44$ & $1.17 \mathrm{E}-04$ & $8.41 \mathrm{E}-13$ & $4.81 \mathrm{E}-04$ & -- & $3.2027 \mathrm{E}-02$ & $2.7346 \mathrm{E}+02$ \\
\hline
\end{tabular}

$\mathrm{CCCG}=8$

\begin{tabular}{|l|l|l|l|l|l|l|l|}
\hline Alpha Factor & 5 th $\%$ & Mean & Median & 95 th $\%$ & MLE & a & b \\
\hline $\boldsymbol{\alpha}_{1}$ & 0.9622170 & 0.9773660 & 0.9783580 & 0.9891370 & -- & $3.1221 \mathrm{E}+02$ & $7.2302 \mathrm{E}+00$ \\
\hline $\boldsymbol{\alpha}_{2}$ & $3.13 \mathrm{E}-03$ & $1.04 \mathrm{E}-02$ & $9.45 \mathrm{E}-03$ & $2.12 \mathrm{E}-02$ & -- & $3.3414 \mathrm{E}+00$ & $3.1609 \mathrm{E}+02$ \\
\hline $\boldsymbol{\alpha}_{3}$ & $6.67 \mathrm{E}-04$ & $5.04 \mathrm{E}-03$ & $4.06 \mathrm{E}-03$ & $1.28 \mathrm{E}-02$ & -- & $1.6130 \mathrm{E}+00$ & $3.1782 \mathrm{E}+02$ \\
\hline $\boldsymbol{\alpha}_{4}$ & $1.86 \mathrm{E}-04$ & $3.26 \mathrm{E}-03$ & $2.30 \mathrm{E}-03$ & $9.62 \mathrm{E}-03$ & -- & $1.0438 \mathrm{E}+00$ & $3.1839 \mathrm{E}+02$ \\
\hline $\boldsymbol{\alpha}_{5}$ & $3.88 \mathrm{E}-05$ & $2.20 \mathrm{E}-03$ & $1.28 \mathrm{E}-03$ & $7.47 \mathrm{E}-03$ & -- & $7.0280 \mathrm{E}-01$ & $3.1873 \mathrm{E}+02$ \\
\hline $\boldsymbol{\alpha}_{6}$ & $5.77 \mathrm{E}-07$ & $1.13 \mathrm{E}-03$ & $3.63 \mathrm{E}-04$ & $4.86 \mathrm{E}-03$ & -- & $3.6184 \mathrm{E}-01$ & $3.1907 \mathrm{E}+02$ \\
\hline $\boldsymbol{\alpha}_{7}$ & $1.19 \mathrm{E}-13$ & $3.98 \mathrm{E}-04$ & $8.44 \mathrm{E}-06$ & $2.25 \mathrm{E}-03$ & -- & $1.2739 \mathrm{E}-01$ & $3.1931 \mathrm{E}+02$ \\
\hline $\boldsymbol{\alpha}_{8}$ & $5.47 \mathrm{E}-36$ & $1.25 \mathrm{E}-04$ & $5.43 \mathrm{E}-11$ & $6.01 \mathrm{E}-04$ & --- & $4.0005 \mathrm{E}-02$ & $3.1940 \mathrm{E}+02$ \\
\hline
\end{tabular}




\section{CCF Parameter Estimations 2015, Section 3}

\section{Section 3.1.1, Generic Demand CCF Prior Distribution: CCF-DEM}

Failure Modes/Fail to Close

Failure Modes/Fail to Load and-or Run

Failure Modes/Fail to Open

Failure Modes/Fail to Open and-or Close

Failure Modes/Fail to Start

Failure Modes/Fail to Stop

Date Range: 1997 through 2015

Total Number of Independent Failure Events: 3598.9

Total Number of Common-Cause Failure Events: 107

ALPHA FACTOR DISTRIBUTIONS

CCCG $=2$
\begin{tabular}{|l|l|l|l|l|l|l|l|}
\hline $\begin{array}{l}\text { Alpha } \\
\text { Factor }\end{array}$ & 5 th\% & Mean & Median & 95 th $\%$ & MLE & a & b \\
\hline $\boldsymbol{\alpha}_{1}$ & 0.9739095 & 0.9802993 & 0.9805202 & 0.9859305 & 0.9804566 & $1.407 \mathrm{E}+03$ & $2.827 \mathrm{E}+01$ \\
\hline $\boldsymbol{\alpha}_{2}$ & $1.41 \mathrm{E}-02$ & $1.97 \mathrm{E}-02$ & $1.95 \mathrm{E}-02$ & $2.61 \mathrm{E}-02$ & $1.95 \mathrm{E}-02$ & $2.827 \mathrm{E}+01$ & $1.407 \mathrm{E}+03$ \\
\hline
\end{tabular}

CCCG $=3$
\begin{tabular}{|l|l|l|l|l|l|l|l|}
\hline $\begin{array}{l}\text { Alpha } \\
\text { Factor }\end{array}$ & 5 th\% & Mean & Median & 95 th\% & MLE & a & b \\
\hline $\boldsymbol{a}_{1}$ & 0.9736531 & 0.9789883 & 0.9791353 & 0.9838123 & 0.9792043 & $2.100 \mathrm{E}+03$ & $4.508 \mathrm{E}+01$ \\
\hline $\mathbf{a}_{2}$ & $1.07 \mathrm{E}-02$ & $1.47 \mathrm{E}-02$ & $1.46 \mathrm{E}-02$ & $1.92 \mathrm{E}-02$ & $1.45 \mathrm{E}-02$ & $3.154 \mathrm{E}+01$ & $2.114 \mathrm{E}+03$ \\
\hline $\boldsymbol{\alpha}_{3}$ & $3.78 \mathrm{E}-03$ & $6.31 \mathrm{E}-03$ & $6.16 \mathrm{E}-03$ & $9.36 \mathrm{E}-03$ & $6.28 \mathrm{E}-03$ & $1.354 \mathrm{E}+01$ & $2.132 \mathrm{E}+03$ \\
\hline
\end{tabular}

CCCG $=4$
\begin{tabular}{|l|l|l|l|l|l|l|l|}
\hline $\begin{array}{l}\text { Alpha } \\
\text { Factor }\end{array}$ & 5 th\% & Mean & Median & 95 th $\%$ & MLE & a & b \\
\hline $\boldsymbol{\alpha}_{1}$ & 0.9736328 & 0.9783076 & 0.9784225 & 0.9826016 & 0.9785984 & $2.786 \mathrm{E}+03$ & $6.177 \mathrm{E}+01$ \\
\hline $\boldsymbol{\alpha}_{2}$ & $1.02 \mathrm{E}-02$ & $1.35 \mathrm{E}-02$ & $1.34 \mathrm{E}-02$ & $1.72 \mathrm{E}-02$ & $1.33 \mathrm{E}-02$ & $3.847 \mathrm{E}+01$ & $2.809 \mathrm{E}+03$ \\
\hline $\boldsymbol{\alpha}_{3}$ & $3.50 \mathrm{E}-03$ & $5.59 \mathrm{E}-03$ & $5.47 \mathrm{E}-03$ & $8.07 \mathrm{E}-03$ & $5.54 \mathrm{E}-03$ & $1.591 \mathrm{E}+01$ & $2.832 \mathrm{E}+03$ \\
\hline $\boldsymbol{\alpha}_{4}$ & $1.25 \mathrm{E}-03$ & $2.60 \mathrm{E}-03$ & $2.48 \mathrm{E}-03$ & $4.34 \mathrm{E}-03$ & $2.56 \mathrm{E}-03$ & $7.393 \mathrm{E}+00$ & $2.840 \mathrm{E}+03$ \\
\hline
\end{tabular}

CCCG $=5$
\begin{tabular}{|l|l|l|l|l|l|l|l|}
\hline $\begin{array}{l}\text { Alpha } \\
\text { Factor }\end{array}$ & 5 th\% & Mean & Median & 95 th\% & MLE & a & b \\
\hline $\boldsymbol{\alpha}_{1}$ & 0.9763661 & 0.9803297 & 0.9804199 & 0.9839886 & 0.9807825 & $3.512 \mathrm{E}+03$ & $7.047 \mathrm{E}+01$ \\
\hline $\boldsymbol{\alpha}_{2}$ & $7.06 \mathrm{E}-03$ & $9.57 \mathrm{E}-03$ & $9.48 \mathrm{E}-03$ & $1.24 \mathrm{E}-02$ & $9.24 \mathrm{E}-03$ & $3.430 \mathrm{E}+01$ & $3.548 \mathrm{E}+03$ \\
\hline $\boldsymbol{\alpha}_{3}$ & $3.87 \mathrm{E}-03$ & $5.78 \mathrm{E}-03$ & $5.69 \mathrm{E}-03$ & $8.01 \mathrm{E}-03$ & $5.67 \mathrm{E}-03$ & $2.072 \mathrm{E}+01$ & $3.562 \mathrm{E}+03$ \\
\hline $\boldsymbol{\alpha}_{4}$ & $1.85 \mathrm{E}-03$ & $3.24 \mathrm{E}-03$ & $3.15 \mathrm{E}-03$ & $4.94 \mathrm{E}-03$ & $3.22 \mathrm{E}-03$ & $1.161 \mathrm{E}+01$ & $3.571 \mathrm{E}+03$ \\
\hline $\boldsymbol{\alpha}_{5}$ & $3.57 \mathrm{E}-04$ & $1.07 \mathrm{E}-03$ & $9.82 \mathrm{E}-04$ & $2.10 \mathrm{E}-03$ & $1.08 \mathrm{E}-03$ & $3.847 \mathrm{E}+00$ & $3.579 \mathrm{E}+03$ \\
\hline
\end{tabular}

CCCG $=6$
\begin{tabular}{|l|l|l|l|l|l|l|l|}
\hline $\begin{array}{l}\text { Alpha } \\
\text { Factor }\end{array}$ & 5 th\% & Mean & Median & 95 th $\%$ & MLE & a & b \\
\hline$\alpha_{1}$ & 0.9784444 & 0.9819123 & 0.9819850 & 0.9851296 & 0.9824132 & $4.209 \mathrm{E}+03$ & $7.753 \mathrm{E}+01$ \\
\hline$\alpha_{2}$ & $5.32 \mathrm{E}-03$ & $7.32 \mathrm{E}-03$ & $7.25 \mathrm{E}-03$ & $9.59 \mathrm{E}-03$ & $7.00 \mathrm{E}-03$ & $3.140 \mathrm{E}+01$ & $4.255 \mathrm{E}+03$ \\
\hline
\end{tabular}

\begin{tabular}{|l|l|l|l|l|l|l|l|}
\hline $\boldsymbol{\alpha}_{3}$ & $3.50 \mathrm{E}-03$ & $5.15 \mathrm{E}-03$ & $5.08 \mathrm{E}-03$ & $7.08 \mathrm{E}-03$ & $5.03 \mathrm{E}-03$ & $2.209 \mathrm{E}+01$ & $4.264 \mathrm{E}+03$ \\
\hline $\boldsymbol{\alpha}_{4}$ & $1.97 \mathrm{E}-03$ & $3.26 \mathrm{E}-03$ & $3.19 \mathrm{E}-03$ & $4.81 \mathrm{E}-03$ & $3.22 \mathrm{E}-03$ & $1.398 \mathrm{E}+01$ & $4.272 \mathrm{E}+03$ \\
\hline $\boldsymbol{\alpha}_{5}$ & $9.19 \mathrm{E}-04$ & $1.85 \mathrm{E}-03$ & $1.77 \mathrm{E}-03$ & $3.05 \mathrm{E}-03$ & $1.85 \mathrm{E}-03$ & $7.937 \mathrm{E}+00$ & $4.278 \mathrm{E}+03$ \\
\hline $\mathbf{a}_{6}$ & $9.39 \mathrm{E}-05$ & $4.94 \mathrm{E}-04$ & $4.18 \mathrm{E}-04$ & $1.15 \mathrm{E}-03$ & $4.88 \mathrm{E}-04$ & $2.115 \mathrm{E}+00$ & $4.284 \mathrm{E}+03$ \\
\hline
\end{tabular}

CCCG $=7$
\begin{tabular}{|l|l|l|l|l|l|l|l|}
\hline $\begin{array}{l}\text { Alpha } \\
\text { Factor }\end{array}$ & 5 th\% & Mean & Median & 95 th\% & MLE & a & b \\
\hline $\boldsymbol{\alpha}_{1}$ & 0.9800577 & 0.9831435 & 0.9832097 & 0.9860157 & 0.9838109 & $4.952 \mathrm{E}+03$ & $8.490 \mathrm{E}+01$ \\
\hline $\boldsymbol{\alpha}_{2}$ & $4.39 \mathrm{E}-03$ & $6.07 \mathrm{E}-03$ & $6.00 \mathrm{E}-03$ & $7.97 \mathrm{E}-03$ & $5.67 \mathrm{E}-03$ & $3.056 \mathrm{E}+01$ & $5.006 \mathrm{E}+03$ \\
\hline $\boldsymbol{\alpha}_{3}$ & $2.86 \mathrm{E}-03$ & $4.24 \mathrm{E}-03$ & $4.18 \mathrm{E}-03$ & $5.85 \mathrm{E}-03$ & $4.07 \mathrm{E}-03$ & $2.138 \mathrm{E}+01$ & $5.015 \mathrm{E}+03$ \\
\hline $\boldsymbol{\alpha}_{4}$ & $1.98 \mathrm{E}-03$ & $3.16 \mathrm{E}-03$ & $3.09 \mathrm{E}-03$ & $4.56 \mathrm{E}-03$ & $3.08 \mathrm{E}-03$ & $1.590 \mathrm{E}+01$ & $5.021 \mathrm{E}+03$ \\
\hline $\mathbf{a}_{5}$ & $1.17 \mathrm{E}-03$ & $2.11 \mathrm{E}-03$ & $2.05 \mathrm{E}-03$ & $3.28 \mathrm{E}-03$ & $2.09 \mathrm{E}-03$ & $1.065 \mathrm{E}+01$ & $5.026 \mathrm{E}+03$ \\
\hline $\boldsymbol{\alpha}_{6}$ & $4.23 \mathrm{E}-04$ & $1.04 \mathrm{E}-03$ & $9.78 \mathrm{E}-04$ & $1.89 \mathrm{E}-03$ & $1.05 \mathrm{E}-03$ & $5.253 \mathrm{E}+00$ & $5.031 \mathrm{E}+03$ \\
\hline $\mathbf{\alpha} 7$ & $1.64 \mathrm{E}-05$ & $2.29 \mathrm{E}-04$ & $1.68 \mathrm{E}-04$ & $6.53 \mathrm{E}-04$ & $2.32 \mathrm{E}-04$ & $1.155 \mathrm{E}+00$ & $5.035 \mathrm{E}+03$ \\
\hline
\end{tabular}

CCCG $=8$
\begin{tabular}{|l|l|l|l|l|l|l|l|}
\hline $\begin{array}{l}\text { Alpha } \\
\text { Factor }\end{array}$ & 5 th\% & Mean & Median & 95 th\% & MLE & $\mathbf{a}$ & $\mathbf{b}$ \\
\hline $\boldsymbol{\alpha}_{1}$ & 0.9813790 & 0.9841756 & 0.9842349 & 0.9867847 & 0.9848780 & $5.661 \mathrm{E}+03$ & $9.103 \mathrm{E}+01$ \\
\hline $\boldsymbol{a}_{2}$ & $3.81 \mathrm{E}-03$ & $5.28 \mathrm{E}-03$ & $5.22 \mathrm{E}-03$ & $6.94 \mathrm{E}-03$ & $4.89 \mathrm{E}-03$ & $3.038 \mathrm{E}+01$ & $5.722 \mathrm{E}+03$ \\
\hline $\boldsymbol{\alpha}_{3}$ & $2.35 \mathrm{E}-03$ & $3.53 \mathrm{E}-03$ & $3.47 \mathrm{E}-03$ & $4.91 \mathrm{E}-03$ & $3.36 \mathrm{E}-03$ & $2.031 \mathrm{E}+01$ & $5.732 \mathrm{E}+03$ \\
\hline $\boldsymbol{a}_{4}$ & $1.76 \mathrm{E}-03$ & $2.79 \mathrm{E}-03$ & $2.74 \mathrm{E}-03$ & $4.03 \mathrm{E}-03$ & $2.70 \mathrm{E}-03$ & $1.607 \mathrm{E}+01$ & $5.736 \mathrm{E}+03$ \\
\hline $\boldsymbol{\alpha}_{5}$ & $1.25 \mathrm{E}-03$ & $2.14 \mathrm{E}-03$ & $2.09 \mathrm{E}-03$ & $3.24 \mathrm{E}-03$ & $2.10 \mathrm{E}-03$ & $1.234 \mathrm{E}+01$ & $5.740 \mathrm{E}+03$ \\
\hline $\mathbf{a}_{5}$ & $6.76 \mathrm{E}-04$ & $1.37 \mathrm{E}-03$ & $1.31 \mathrm{E}-03$ & $2.26 \mathrm{E}-03$ & $1.36 \mathrm{E}-03$ & $7.870 \mathrm{E}+00$ & $5.745 \mathrm{E}+03$ \\
\hline $\boldsymbol{\alpha}_{7}$ & $1.77 \mathrm{E}-04$ & $5.88 \mathrm{E}-04$ & $5.32 \mathrm{E}-04$ & $1.19 \mathrm{E}-03$ & $5.90 \mathrm{E}-04$ & $3.384 \mathrm{E}+00$ & $5.749 \mathrm{E}+03$ \\
\hline $\boldsymbol{\alpha}_{8}$ & $1.84 \mathrm{E}-06$ & $1.18 \mathrm{E}-04$ & $6.75 \mathrm{E}-05$ & $4.06 \mathrm{E}-04$ & $1.17 \mathrm{E}-04$ & $6.793 \mathrm{E}-01$ & $5.752 \mathrm{E}+03$ \\
\hline
\end{tabular}




\section{CCF Parameter Estimations 2015, Section 3}

\section{Section 3.1.2, Generic Rate CCF Prior Distribution: CCF-RATE}

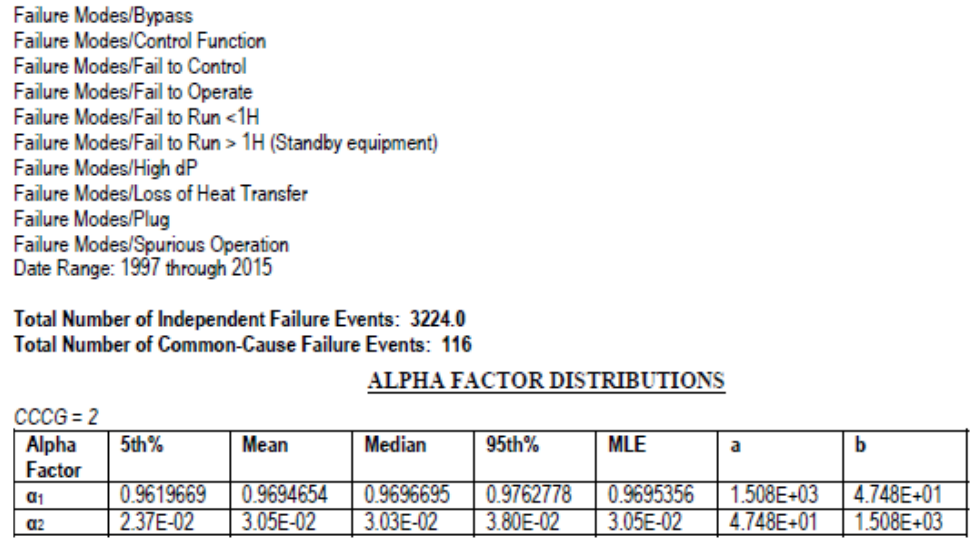

CCCG $=3$
\begin{tabular}{|l|l|l|l|l|l|l|l|}
\hline $\begin{array}{l}\text { Alpha } \\
\text { Factor }\end{array}$ & 5 th\% & Mean & Median & 95 th\% & MLE & a & b \\
\hline $\boldsymbol{\alpha}_{1}$ & 0.9646529 & 0.9706436 & 0.9707767 & 0.9761762 & 0.9707316 & $2.246 \mathrm{E}+03$ & $6.792 \mathrm{E}+01$ \\
\hline $\boldsymbol{\alpha}_{2}$ & $1.33 \mathrm{E}-02$ & $1.75 \mathrm{E}-02$ & $1.74 \mathrm{E}-02$ & $2.22 \mathrm{E}-02$ & $1.74 \mathrm{E}-02$ & $4.050 \mathrm{E}+01$ & $2.273 \mathrm{E}+03$ \\
\hline $\boldsymbol{\alpha}_{3}$ & $8.41 \mathrm{E}-03$ & $1.19 \mathrm{E}-02$ & $1.17 \mathrm{E}-02$ & $1.58 \mathrm{E}-02$ & $1.19 \mathrm{E}-02$ & $2.743 \mathrm{E}+01$ & $2.286 \mathrm{E}+03$ \\
\hline
\end{tabular}

CCCG $=4$
\begin{tabular}{|l|l|l|l|l|l|l|l|}
\hline $\begin{array}{l}\text { Alpha } \\
\text { Factor }\end{array}$ & 5 th\% & Mean & Median & 95 th\% & MLE & a & b \\
\hline $\boldsymbol{\alpha}_{1}$ & 0.9678618 & 0.9728563 & 0.9729563 & 0.9775041 & 0.9730394 & $2.982 \mathrm{E}+03$ & $8.320 \mathrm{E}+01$ \\
\hline $\boldsymbol{\alpha}_{2}$ & $9.75 \mathrm{E}-03$ & $1.29 \mathrm{E}-02$ & $1.28 \mathrm{E}-02$ & $1.64 \mathrm{E}-02$ & $1.27 \mathrm{E}-02$ & $3.960 \mathrm{E}+01$ & $3.025 \mathrm{E}+03$ \\
\hline $\boldsymbol{\alpha}_{3}$ & $6.19 \mathrm{E}-03$ & $8.77 \mathrm{E}-03$ & $8.66 \mathrm{E}-03$ & $1.17 \mathrm{E}-02$ & $8.77 \mathrm{E}-03$ & $2.688 \mathrm{E}+01$ & $3.038 \mathrm{E}+03$ \\
\hline $\boldsymbol{\alpha} 4$ & $3.47 \mathrm{E}-03$ & $5.46 \mathrm{E}-03$ & $5.35 \mathrm{E}-03$ & $7.81 \mathrm{E}-03$ & $5.47 \mathrm{E}-03$ & $1.672 \mathrm{E}+01$ & $3.048 \mathrm{E}+03$ \\
\hline
\end{tabular}

CCCG $=5$
\begin{tabular}{|l|l|l|l|l|l|l|l|}
\hline $\begin{array}{l}\text { Alpha } \\
\text { Factor }\end{array}$ & 5 th\% & Mean & Median & 95 th\% & MLE & a & b \\
\hline $\boldsymbol{\alpha}_{1}$ & 0.9716549 & 0.9758620 & 0.9759421 & 0.9797885 & 0.9761639 & $3.756 \mathrm{E}+03$ & $9.290 \mathrm{E}+01$ \\
\hline $\boldsymbol{\alpha}_{2}$ & $6.59 \mathrm{E}-03$ & $8.93 \mathrm{E}-03$ & $8.85 \mathrm{E}-03$ & $1.16 \mathrm{E}-02$ & $8.61 \mathrm{E}-03$ & $3.438 \mathrm{E}+01$ & $3.814 \mathrm{E}+03$ \\
\hline $\boldsymbol{\alpha}_{3}$ & $5.33 \mathrm{E}-03$ & $7.46 \mathrm{E}-03$ & $7.37 \mathrm{E}-03$ & $9.88 \mathrm{E}-03$ & $7.40 \mathrm{E}-03$ & $2.870 \mathrm{E}+01$ & $3.820 \mathrm{E}+03$ \\
\hline $\boldsymbol{\alpha}_{4}$ & $3.14 \mathrm{E}-03$ & $4.81 \mathrm{E}-03$ & $4.73 \mathrm{E}-03$ & $6.78 \mathrm{E}-03$ & $4.84 \mathrm{E}-03$ & $1.852 \mathrm{E}+01$ & $3.830 \mathrm{E}+03$ \\
\hline $\boldsymbol{\alpha}_{5}$ & $1.66 \mathrm{E}-03$ & $2.94 \mathrm{E}-03$ & $2.85 \mathrm{E}-03$ & $4.50 \mathrm{E}-03$ & $2.99 \mathrm{E}-03$ & $1.130 \mathrm{E}+01$ & $3.837 \mathrm{E}+03$ \\
\hline
\end{tabular}

CCCG $=6$
\begin{tabular}{|l|l|l|l|l|l|l|l|}
\hline $\begin{array}{l}\text { Alpha } \\
\text { Factor }\end{array}$ & 5 th\% & Mean & Median & 95 th\% & MLE & a & b \\
\hline $\mathbf{a}_{1}$ & 0.9746515 & 0.9783006 & 0.9783677 & 0.9817130 & 0.9786685 & $4.501 \mathrm{E}+03$ & $9.984 \mathrm{E}+01$ \\
\hline
\end{tabular}

\begin{tabular}{|l|l|l|l|l|l|l|l|}
\hline $\mathbf{a}_{2}$ & $4.55 \mathrm{E}-03$ & $6.34 \mathrm{E}-03$ & $6.27 \mathrm{E}-03$ & $8.38 \mathrm{E}-03$ & $6.02 \mathrm{E}-03$ & $2.919 \mathrm{E}+01$ & $4.572 \mathrm{E}+03$ \\
\hline $\mathbf{\alpha}_{3}$ & $4.42 \mathrm{E}-03$ & $6.19 \mathrm{E}-03$ & $6.12 \mathrm{E}-03$ & $8.20 \mathrm{E}-03$ & $6.10 \mathrm{E}-03$ & $2.847 \mathrm{E}+01$ & $4.572 \mathrm{E}+03$ \\
\hline $\mathbf{a}_{4}$ & $3.16 \mathrm{E}-03$ & $4.69 \mathrm{E}-03$ & $4.62 \mathrm{E}-03$ & $6.46 \mathrm{E}-03$ & $4.68 \mathrm{E}-03$ & $2.158 \mathrm{E}+01$ & $4.579 \mathrm{E}+03$ \\
\hline $\mathbf{a}_{5}$ & $1.48 \mathrm{E}-03$ & $2.58 \mathrm{E}-03$ & $2.51 \mathrm{E}-03$ & $3.92 \mathrm{E}-03$ & $2.60 \mathrm{E}-03$ & $1.187 \mathrm{E}+01$ & $4.589 \mathrm{E}+03$ \\
\hline $\mathbf{a}_{6}$ & $9.78 \mathrm{E}-04$ & $1.90 \mathrm{E}-03$ & $1.83 \mathrm{E}-03$ & $3.06 \mathrm{E}-03$ & $1.93 \mathrm{E}-03$ & $8.727 \mathrm{E}+00$ & $4.592 \mathrm{E}+03$ \\
\hline
\end{tabular}

CCCG $=7$
\begin{tabular}{|l|l|l|l|l|l|l|l|}
\hline $\begin{array}{l}\text { Alpha } \\
\text { Factor }\end{array}$ & 5 th\% & Mean & Median & 95 th\% & MLE & a & $b$ \\
\hline $\boldsymbol{\alpha}_{1}$ & 0.9771278 & 0.9803354 & 0.9803974 & 0.9833355 & 0.9808535 & $5.294 \mathrm{E}+03$ & $1.062 \mathrm{E}+02$ \\
\hline $\boldsymbol{\alpha}_{2}$ & $3.51 \mathrm{E}-03$ & $4.98 \mathrm{E}-03$ & $4.91 \mathrm{E}-03$ & $6.65 \mathrm{E}-03$ & $4.56 \mathrm{E}-03$ & $2.687 \mathrm{E}+01$ & $5.373 \mathrm{E}+03$ \\
\hline $\boldsymbol{\alpha}_{3}$ & $3.25 \mathrm{E}-03$ & $4.67 \mathrm{E}-03$ & $4.60 \mathrm{E}-03$ & $6.29 \mathrm{E}-03$ & $4.52 \mathrm{E}-03$ & $2.519 \mathrm{E}+01$ & $5.375 \mathrm{E}+03$ \\
\hline $\boldsymbol{\alpha}_{4}$ & $2.85 \mathrm{E}-03$ & $4.18 \mathrm{E}-03$ & $4.12 \mathrm{E}-03$ & $5.72 \mathrm{E}-03$ & $4.14 \mathrm{E}-03$ & $2.257 \mathrm{E}+01$ & $5.378 \mathrm{E}+03$ \\
\hline $\boldsymbol{\alpha}_{5}$ & $1.95 \mathrm{E}-03$ & $3.07 \mathrm{E}-03$ & $3.01 \mathrm{E}-03$ & $4.41 \mathrm{E}-03$ & $3.09 \mathrm{E}-03$ & $1.660 \mathrm{E}+01$ & $5.384 \mathrm{E}+03$ \\
\hline $\boldsymbol{\alpha}_{6}$ & $1.02 \mathrm{E}-03$ & $1.87 \mathrm{E}-03$ & $1.81 \mathrm{E}-03$ & $2.93 \mathrm{E}-03$ & $1.90 \mathrm{E}-03$ & $1.009 \mathrm{E}+01$ & $5.390 \mathrm{E}+03$ \\
\hline $\boldsymbol{\alpha}_{7}$ & $3.50 \mathrm{E}-04$ & $9.02 \mathrm{E}-04$ & $8.41 \mathrm{E}-04$ & $1.66 \mathrm{E}-03$ & $9.30 \mathrm{E}-04$ & $4.872 \mathrm{E}+00$ & $5.395 \mathrm{E}+03$ \\
\hline
\end{tabular}

CCCG $=8$
\begin{tabular}{|l|l|l|l|l|l|l|l|}
\hline $\begin{array}{l}\text { Alpha } \\
\text { Factor }\end{array}$ & 5 th\% & Mean & Median & 95 th\% & MLE & $\mathbf{a}$ & $\mathbf{b}$ \\
\hline $\mathbf{a}_{1}$ & 0.9790895 & 0.9819652 & 0.9820155 & 0.9646638 & 0.9825346 & $6.054 \mathrm{E}+03$ & $1.112 \mathrm{E}+02$ \\
\hline $\mathbf{a}_{2}$ & $2.94 \mathrm{E}-03$ & $4.20 \mathrm{E}-03$ & $4.14 \mathrm{E}-03$ & $5.63 \mathrm{E}-03$ & $3.79 \mathrm{E}-03$ & $2.587 \mathrm{E}+01$ & $6.139 \mathrm{E}+03$ \\
\hline $\mathbf{a}_{3}$ & $2.34 \mathrm{E}-03$ & $3.47 \mathrm{E}-03$ & $3.42 \mathrm{E}-03$ & $4.79 \mathrm{E}-03$ & $3.31 \mathrm{E}-03$ & $2.140 \mathrm{E}+01$ & $6.144 \mathrm{E}+03$ \\
\hline $\mathbf{a}_{4}$ & $2.42 \mathrm{E}-03$ & $3.57 \mathrm{E}-03$ & $3.52 \mathrm{E}-03$ & $4.91 \mathrm{E}-03$ & $3.52 \mathrm{E}-03$ & $2.204 \mathrm{E}+01$ & $6.143 \mathrm{E}+03$ \\
\hline $\mathbf{a}_{5}$ & $1.92 \mathrm{E}-03$ & $2.96 \mathrm{E}-03$ & $2.91 \mathrm{E}-03$ & $4.18 \mathrm{E}-03$ & $2.96 \mathrm{E}-03$ & $1.826 \mathrm{E}+01$ & $6.147 \mathrm{E}+03$ \\
\hline $\mathbf{a}_{6}$ & $1.31 \mathrm{E}-03$ & $2.19 \mathrm{E}-03$ & $2.13 \mathrm{E}-03$ & $3.25 \mathrm{E}-03$ & $2.21 \mathrm{E}-03$ & $1.348 \mathrm{E}+01$ & $6.152 \mathrm{E}+03$ \\
\hline $\mathbf{a}_{7}$ & $5.53 \mathrm{E}-04$ & $1.16 \mathrm{E}-03$ & $1.11 \mathrm{E}-03$ & $1.96 \mathrm{E}-03$ & $1.19 \mathrm{E}-03$ & $7.177 \mathrm{E}+00$ & $6.158 \mathrm{E}+03$ \\
\hline $\mathbf{a}_{3}$ & $1.30 \mathrm{E}-04$ & $4.81 \mathrm{E}-04$ & $4.29 \mathrm{E}-04$ & $1.01 \mathrm{E}-03$ & $4.94 \mathrm{E}-04$ & $2.968 \mathrm{E}+00$ & $6.162 \mathrm{E}+03$ \\
\hline
\end{tabular}




\section{APPENDIX B}

\section{EXAMPLE OF HOW TO PERFORM A BAYESIAN UPDATE ON CCF PARAMETERS USING PRIOR DISTRIBUTIONS}

This appendix presents an example of how to perform a Bayesian update on CCF parameters (specifically, the alpha factors, as in the Alpha Factor Model) using the prior distributions.

CCF SPAR Rules $2015 \rightarrow 2.1 .1 .1$ ALL-MDP-FS is used as the example (see Figure B-1): Go to the RADS/CCF website https://rads.inl.gov/Pages/CCF.aspx; click the CCF Rules tab on the left side; select SPAR Rules $2015 \rightarrow 2$ 1.1.1 ALL-MDP-FS; and run the rule by clicking the Run Rule tab on the bottom.

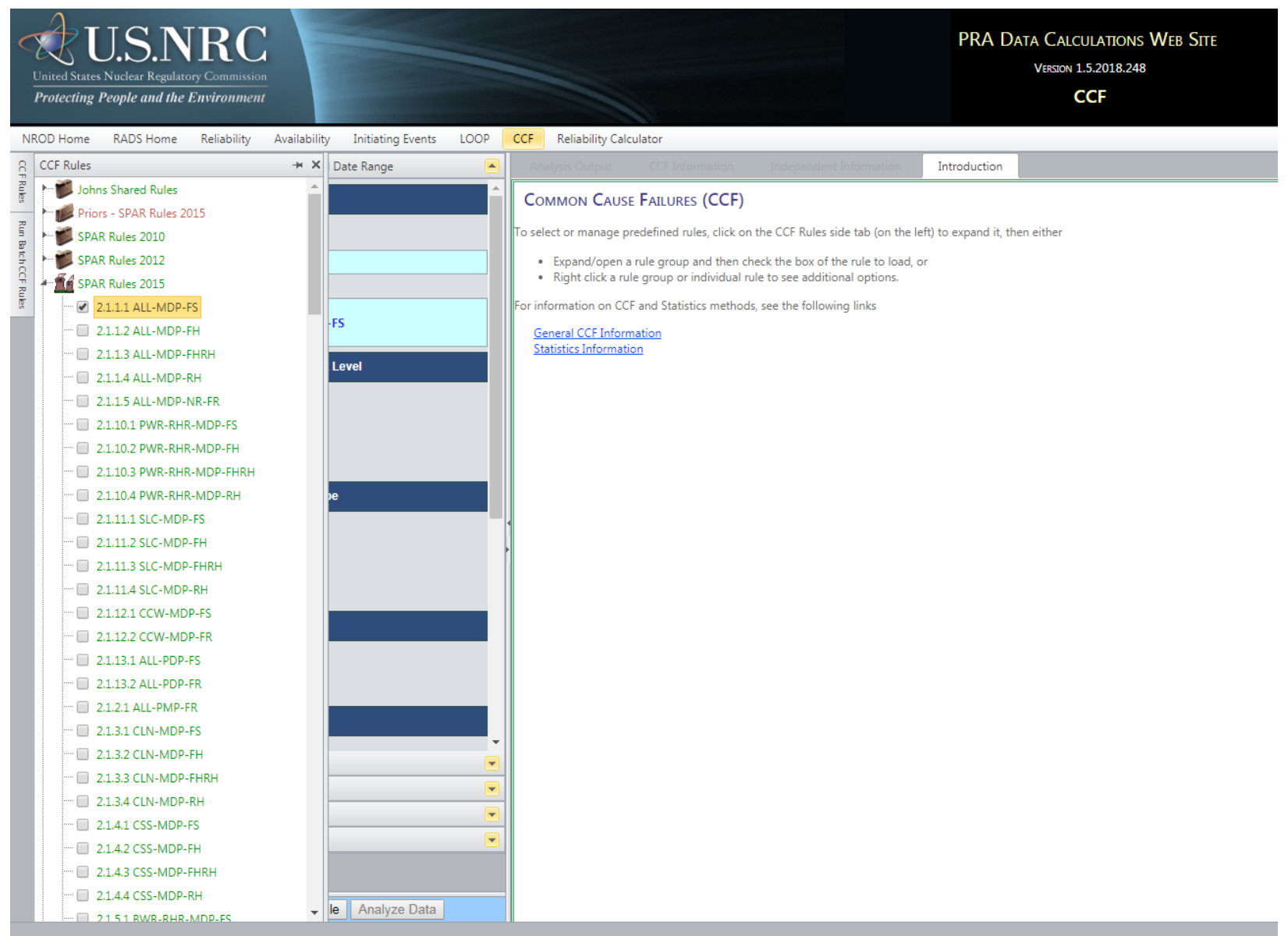

Maintained for the Nuclear Regulatory Commission by Idaho National Laboratory.

Figure B-1. Run CCF Rule ALL-MDP-FS. 
The alpha factor results will be displayed as in Figure B-2.

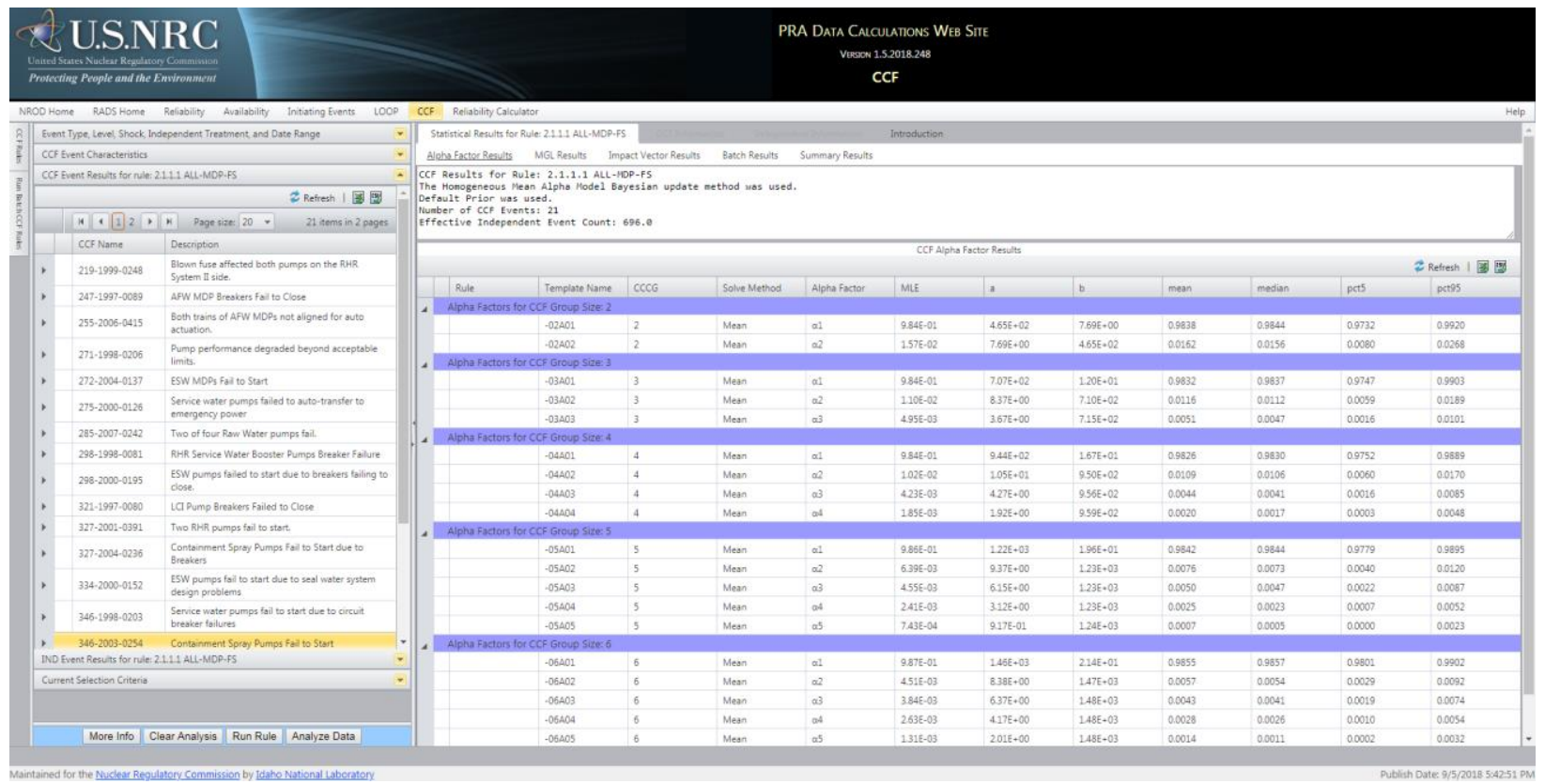

Figure B-2. CCF Alpha Factor Results for ALL-MDP-FS.

Now go to the Impact Vector Results tab.

For $\mathbf{C C C G}=\mathbf{2}$, it shows:

Adjusted Independent Count $n(I)=442.91$

$$
\begin{aligned}
& \mathrm{n} 1=12.262 \\
& \mathrm{n} 2=7.2523
\end{aligned}
$$

So $\sum \mathrm{n}=\mathrm{n}(\mathrm{I})+\mathrm{n} 1+\mathrm{n} 2=462.4243$

One can then obtain the MLE of the alpha factors:

$$
\begin{aligned}
& \alpha_{1}=[n(I)+n 1] / \sum_{i} n=\frac{442.91+12.262}{462.4243}=0.9843 \\
& \alpha_{2}=n 2 / \sum_{i} n=7.2523 / 462.4243=0.0157
\end{aligned}
$$

The current default prior in the database is the 2005 Prior (see Appendix A) ${ }^{\mathrm{d}}$, which has the following distribution parameters for $\mathrm{CCCG}=2$ :

$$
\begin{aligned}
\alpha 1: & a 1=10.246 \\
b_{1} & =0.43452 \\
\alpha_{2}: a_{2} & =0.43452 \\
b_{2} & =10.246
\end{aligned}
$$

The posterior distribution parameters can be calculated as:

$$
\alpha 1: a 1^{\prime}=a 1+n(I)+n 1=465.418
$$

\footnotetext{
${ }^{\mathrm{d}}$ Note the CCF database now includes a selection of prior distributions to be used, under the tab Event Type, Level, Shock, Independent Treatment, and Date Range -> Prior Distribution. The 2005 Priors are called "Default," the new priors developed in Section 3 are "Default 2015," and the causal priors developed in Section 4 are "Component," "Design," "Environment," "Human," and "Other."
} 
$\mathrm{b}^{\prime}{ }^{\prime}=\mathrm{b} 1+\mathrm{n} 2=7.68682$

The mean of $\alpha 1=\mathrm{a} 1^{\prime} /\left(\mathrm{a} 1^{\prime}+\mathrm{b} 1^{\prime}\right)=0.9838$

$\alpha 2: a 2^{\prime}=a 2+n 2=7.6868$

$\mathrm{b}_{2}{ }^{\prime}=\mathrm{b}_{2}+\mathrm{n}(\mathrm{I})+\mathrm{n} 1=465.418$

The mean of $\alpha_{2}=a_{2}{ }^{\prime} /\left(a_{2}{ }^{\prime}+b_{2}{ }^{\prime}\right)=0.0162$

For $\mathbf{C C C G}=\mathbf{3}$, the Impact Vector Results tab shows:

Adjusted Independent Count $n(I)=664.36$

$$
\begin{aligned}
& \mathrm{n} 1=12.856 \\
& \mathrm{n} 2=7.5369 \\
& \mathrm{n} 3=3.4067
\end{aligned}
$$

So $\sum \mathrm{n}=\mathrm{n}(\mathrm{I})+\mathrm{n} 1+\mathrm{n} 2+\mathrm{n} 3=688.1596$

One can then obtain the MLE of the alpha factors:

$$
\begin{aligned}
& \alpha_{1}=[n(I)+n 1] / \sum_{i} n=\frac{664.36+12.856}{688.1596}=0.98410 \\
& \alpha_{2}=n 2 / \sum_{i} n=7.5369 / 688.1596=0.01095 \\
& \alpha_{3}=n 3 / \sum_{i} n=3.4067 / 688.1596=0.00495
\end{aligned}
$$

The 2005 Prior has the following distribution parameters for $\mathrm{CCCG}=3$ :
$\alpha_{1}: a_{1}=29.555$
$\mathrm{b}_{1}=1.1008$
$\alpha_{2}: a_{2}=0.83366$
$\mathrm{b}_{2}=29.822$
$\alpha_{3}: a_{3}=0.26722$
$\mathrm{b}_{3}=30.388$

The posterior distribution parameters can be calculated as:

$\begin{aligned} \alpha_{1}: & a_{1}{ }^{\prime}=a_{1}+n(I)+n 1=706.771 \\ b_{1}{ }^{\prime} & =b_{1}+n 2+n 3=12.0444\end{aligned}$

The mean of $\alpha_{1}=a_{1}{ }^{\prime} /\left(a_{1}^{\prime}+b_{1}^{\prime}\right)=0.98324$

$\alpha_{2}: a_{2}{ }^{\prime}=a_{2}+n 2=8.37056$

$\mathrm{b}_{2}{ }^{\prime}=\mathrm{b}_{2}+\mathrm{n}(\mathrm{I})+\mathrm{n} 1+\mathrm{n} 3=710.4447$

The mean of $\alpha_{2}=a_{2}{ }^{\prime} /\left(a_{2}{ }^{\prime}+b_{2}{ }^{\prime}\right)=0.01165$

$$
\begin{aligned}
\alpha_{3}: a_{3}{ }^{\prime} & =a_{3}+n 3=3.67392 \\
b_{3}{ }^{\prime} & =b_{3}+n(I)+n 1+n 2=715.1409
\end{aligned}
$$

The mean of $\alpha_{3}=a_{3}{ }^{\prime} /\left(a_{3}{ }^{\prime}+b_{3}{ }^{\prime}\right)=0.00511$

The above hand calculation results (MLE and mean of alpha factors $\alpha_{k}$, and posterior distribution parameters $a_{\mathrm{k}}$ ' and $\mathrm{b}_{\mathrm{k}}{ }^{\prime}$ ) were checked against and found identical to those in the Alpha Factor Results tab. 


\begin{tabular}{|c|c|c|c|c|c|c|c|}
\hline Template Name & $\mathrm{CCCG}$ & Solve Method & Alpha Factor & MLE & a & b & mean \\
\hline \multicolumn{8}{|l|}{ CF Group Size: 2} \\
\hline$-02 \mathrm{~A} 01$ & 2 & Mean & $\alpha 1$ & $9.84 \mathrm{E}-01$ & $4.65 E+02$ & $7.69 \mathrm{E}+00$ & 0.9838 \\
\hline$-02 \mathrm{~A} 02$ & 2 & Mean & $\alpha 2$ & $1.57 \mathrm{E}-02$ & $7.69 \mathrm{E}+00$ & $4.65 E+02$ & 0.0162 \\
\hline \multicolumn{8}{|l|}{ CF Group Size: 3} \\
\hline$-03 \mathrm{~A} 01$ & 3 & Mean & $\alpha 1$ & $9.84 \mathrm{E}-01$ & $7.07 \mathrm{E}+02$ & $1.20 E+01$ & 0.9832 \\
\hline$-03 \mathrm{~A} 02$ & 3 & Mean & $\alpha 2$ & $1.10 \mathrm{E}-02$ & $8.37 E+00$ & $7.10 E+02$ & 0.0116 \\
\hline$-03 \mathrm{~A} 03$ & 3 & Mean & $\alpha 3$ & 4.95E-03 & $3.67 \mathrm{E}+00$ & $7.15 E+02$ & 0.0051 \\
\hline
\end{tabular}

Figure B-3. ALL-MDP-FS CCF Alpha Factor Results for CCCG of 2 and 3. 


\section{APPENDIX C}

\section{NEW PROCESS TO ESTIMATE MAPPING UP FACTOR \\ $\rho$}

\section{Background}

Proposed work for a generic prior for the alpha factors requires mapping up among various commoncause group sizes. For this, the Binomial Failure Rate (BFR) parameter (or mapping up factor) $\rho$ must be estimated. No estimate is given in Ref. [C-1], only some advice as to when $\rho$ should be "small" or "large." The maximum likelihood estimate (MLE) of $\rho$ can be found numerically using the work given in this appendix. Alternatively, the Method of Moments (MM) can be used to estimate $\rho$.

Consider the data from system sizes $m_{i}$ (Impact vectors, as distinguished from raw data, are discussed at the very end of this appendix.) Ref. [C-1] suggests using a different $\rho$ for each event. However, for a generic prior, we will use the data from many group sizes together. The MLE will be found using all the data together. The Method of Moments will obtain a separate $\rho$ for each group size $m$, combining all the data with that $m$ to obtain $\rho$. Further work will be needed to help decide which method is preferable.

The following restrictions limit the data that should be used.

- Lethal events are not relevant. In such events, occurrence of the cause guarantees that all components fail, so $\rho$ must equal to 1 . However, nonlethal events can be used even if all $m$ components happen to fail, and the formula derived below can be used for nonlethal events.

- Of course, events with no failed components cannot be used. Also, events with exactly one failed component are for the most part classified as independent failures, even if they may have potential CCF linkages to other components. Therefore, only events with 2 or more failed components give trustworthy information about $\rho$. In summary, the values of counts $n_{2}{ }^{(m)}$ through $n_{m}{ }^{(m)}$ should be used, excluding the single failures and the lethal events. In the notation here, $m$ in parentheses is an index, showing dependence on $m$; it does not mean "to the $m$ th power".

- Data from a group of size 2 give no information about $\rho$, because the rules of the above bullet would force every included event to have 2 failed components, regardless of $\rho$.

- If the data have no triple failures or higher, that is $n_{k}^{(m)}=0$ for $k>2$, it will be seen below that $\rho$ is estimated as 0 , whether the MLE or the MM is used.

The derivations below treat the numbers of failed components as known. A discussion at the end of this appendix shows how to use these formulas even when the numbers are estimated by impact vectors.

\section{Maximum Likelihood Estimation of $\rho$}

For each $m>2$, let $n_{k}^{(m)}$ be the number of events with $k$ failed components, $2 \leq k \leq m$. The work below uses all the data, from varying group sizes $m$. If it is desired to use only one $m$, simply ignore the data from other groups.

To estimate $\rho$, treat the data as if generated from the BFR model, with the same $\rho$ for every event. That is, let $X$ have a $\operatorname{binomial}(m, \rho)$ distribution, and exclude the possibilities that $X=0$ or 1 . The probability that one of these common cause (CC) events has $k$ failed components is, by the binomial formula, 
$\operatorname{Pr}(X=k \mid X \geq 2)=\operatorname{Pr}(X=k) / \operatorname{Pr}(X \geq 2)$

$$
=\left(\begin{array}{c}
m \\
k
\end{array}\right) \rho^{k}(1-\rho)^{m-k} /\left[1-(1-\rho)^{m}-m \rho(1-\rho)^{m-1}\right] .
$$

To eliminate some clutter below, define

$P 2^{(m)}=\left\lfloor 1-(1-\rho)^{m}-m \rho(1-\rho)^{m-1}\right\rfloor$.

The interpretation of $P 2^{(m)}$ is the probability that a CC shock causes at least 2 failed components. It is worth noting that

$$
\frac{\partial}{\partial \rho} P 2^{(m)}=m(1-\rho)^{m-1}-m(1-\rho)^{m-1}+m \rho(m-1)(1-\rho)^{m-2}=m(m-1) \rho(1-\rho)^{m-2} .
$$

Let $n_{k}^{(m)}$ be the number of events with $k$ failed components in a group of size $m$. Then the probability that $n_{k}^{(m)}$ particular events had $k$ failed components is given by Eq. C-1 raised to the $n_{k}^{(m)}$ power.

Therefore, the likelihood is

$L=\prod_{m>2} \prod_{k=2}^{m}[\operatorname{Pr}(X=k \mid X \geq 2)]^{n_{k}^{(m)}}$.

The logarithm of this, after collecting similar terms, is

$$
\ln (L)=C+\left[\sum_{m}\left(\sum_{k=2}^{m} k n_{k}{ }^{(m)}\right)\right] \ln (\rho /(1-\rho))+\left[\sum_{m} m\left(\sum_{k=2}^{m} n_{k}{ }^{(m)}\right)\right] \ln (1-\rho)-\sum_{m}\left[\ln \left(P 2^{(m)}\right) \sum_{k=2}^{m} n_{k}{ }^{(m)}\right],
$$

where $C$ includes the binomial coefficients, which do not depend on $\rho$.

This can be rewritten more compactly as

$$
\ln (L)=C+C F \ln (\rho /(1-\rho))+C A R \ln (1-\rho)-\sum_{m>2}\left[\ln \left(P 2^{(m)}\right) n_{T}^{(m)}\right],
$$

where

$C F=$ number of component failures,

$C A R=$ number of components at risk, i.e. components in groups where failure events occurred, $n_{T}^{(m)}=$ total number of failure events in groups of size $m$.

It follows, making use of Eq. C-3 that

$$
\begin{aligned}
\frac{\partial}{\partial \rho} \ln (L) & =\frac{C F}{\rho}+\frac{C F}{1-\rho}-\frac{C A R}{1-\rho}-\sum_{m}\left[n_{T}{ }^{(m)} \frac{m(m-1) \rho(1-\rho)^{m-2}}{P 2^{(m)}}\right] \\
& =\frac{C F-\rho C A R}{\rho(1-\rho)}-\rho \sum_{m}\left[n_{T}{ }^{(m)} \frac{m(m-1)(1-\rho)^{m-2}}{P 2^{(m)}}\right]
\end{aligned}
$$

Note first that if $\rho$ is as large as $C F / C A R$ or more, then the derivative (4) is negative. Therefore, $\ln (L)$ can only be maximized by a value of $\rho$ between $C F / C A R$ and 0 . To examine the behavior for $\rho$ near 0 , recall that $\mathrm{P} 2^{(m)}=\operatorname{Pr}(X \geq 2)$, Based on the elements of a binomial distribution, observe that as $\rho \rightarrow 0$, $\operatorname{Pr}(X \geq 2) / \operatorname{Pr}(X=2) \rightarrow 1$. $\operatorname{Pr}(X=2)$ is equal to, 


$$
\frac{m(m-1)}{2} \rho^{2}(1-\rho)^{m-2}
$$

Substitute this into Eq. C-4 to see that, as $\rho \rightarrow 0$

$$
\begin{aligned}
\frac{\partial}{\partial \rho} \ln (L) & \approx \frac{C F-\rho C A R}{\rho(1-\rho)}-\rho \sum_{m}\left[n_{T}{ }^{(m)} \frac{2}{\rho^{2}}\right] \\
& =\frac{C F-\rho C A R}{\rho(1-\rho)}-\frac{2 n_{T O T}}{\rho} \\
& =\frac{C F-2 n_{T O T}-\rho C A R+\rho 2 n_{T O T}}{\rho(1-\rho)} .
\end{aligned}
$$

In the special case with only double failures, the number of failed components equals twice the number of failure events, that is, $C F=2 n_{\text {Tот. }}$. Therefore,

$\frac{\partial}{\partial \rho} \ln (L) \approx \frac{-C A R+2 n_{T O T}}{1-\rho}<0$.

Because the derivative is negative in this special case, the MLE of $\rho$ is 0.

In the more general case with $C F>2 n_{T O T}$, Eq. C-5 yields

$\frac{\partial}{\partial \rho} \ln (L) \approx \frac{C F-2 n_{T O T}}{\rho}>0$.

Note, the sign is now positive. In conclusion, if the data contain at least one event with more than two failed components, the derivative of Eq. $\mathrm{C}-4$ is positive for $\rho$ near 0 and negative for $\rho=C F / C A R$. The exact value of $\rho$ that makes the derivative equal to 0 is the MLE. It must be found numerically. (This discussion has avoided the question of whether there are multiple solutions; that should be checked during the process of finding a numerical solution.)

An issue that has not yet been considered is the uncertainty in $\rho$. If we used observed raw data, the second derivative of $\ln (L)$ could, in principle, be used to find the asymptotic variance of $\rho$. However, the use of impact vectors instead of pure data will greatly complicate this approach.

\section{Method of Moments for Estimating $\rho$}

Kvam [C-2] presents a method of moments (MM) estimator for $\rho$. However, his method assumes that single failures can be accurately classified as either independent or common cause, unlike the present situation. Therefore, Kvam's method will need modification.

His approach is as follows. Let $U$ be the number of failed components during a nonlethal common cause event, except $U=0$ can never be observed. Therefore, $U$ is a binomial $(m, \rho)$ random variable truncated to always have values $\geq 1$. It is a moderately straightforward calculation to show that

$$
\frac{E[U(U-1)]}{(m-1) E(U)}=\rho \text {. }
$$

The expectations (or moments) on the left can be estimated from the data. Using the notation for $n_{k}{ }^{(m)}$ defined just below Eq. C-3, estimate $\operatorname{Pr}(U=k)$ by $n_{k}{ }^{(m)} / n_{T}{ }^{(m)}$. Here, $n_{T}$ is the sum of the $n_{k}$ s. Then the ratio on the left side of Eq. C-6 is estimated by

$\frac{\sum_{k=1}^{m} k(k-1) n_{k}{ }^{(m)}}{(m-1) \sum_{k=1}^{m} k n_{k}{ }^{(m)}}$. 
This is Kvam's estimate of $\rho$, for any one value of $m$.

This method can be adapted as follows to the INL situation, in which single failures are generally not identified as common cause. For the INL applications, let $U$ be the number of failed components during a nonlethal common cause event, except $U \leq 1$ is not observed. Therefore, $U$ is a $\operatorname{binomial}(m, \rho)$ random variable truncated to always have values $\geq 2$. This is consistent with bullet 2 at the start of this note.

Now the moments must be calculated. Let $V$ be a binomial $(m, \rho)$, which can be thought of as $U$ if the values 0 and 1 could be observed. The distribution of $U$ is given by

$$
\begin{aligned}
\operatorname{Pr}(U=k) & =\operatorname{Pr}(V=k \mid V \geq 2) \\
& =\operatorname{Pr}(V=k) / \operatorname{Pr}(V \geq 2) \\
& =\operatorname{Pr}(V=k) / \mathrm{P} 2^{(m)},
\end{aligned}
$$

where $\mathrm{P} 2^{(m)}$ is defined by Eq. C-2.

Therefore

$$
\begin{aligned}
E(U) & =\sum_{k=2}^{m} k \operatorname{Pr}(U=k) \\
& =\frac{\sum_{k=2}^{m} k \operatorname{Pr}(V=k)}{\mathrm{P} 2^{(m)}} \\
& =\frac{\sum_{k=0}^{m} k \operatorname{Pr}(V=k)-\sum_{k=0}^{1} k \operatorname{Pr}(V=k)}{\mathrm{P} 2^{(m)}} \\
& =\frac{E(V)-\operatorname{Pr}(V=1)}{\mathrm{P} 2^{(m)}} \\
& =\frac{m \rho-m \rho(1-\rho)^{m-1}}{\mathrm{P} 2^{(m)}} .
\end{aligned}
$$

In similar manner, it can be shown that

$$
\begin{aligned}
E[U(U-1)] & =E\left(U^{2}\right)-E(U) \\
& =\frac{E\left(V^{2}\right)-\operatorname{Pr}(V=1)}{\mathrm{P} 2^{(m)}}-\frac{E(V)-\operatorname{Pr}(V=1)}{\mathrm{P} 2^{(m)}} \\
& =\frac{E\left(V^{2}\right)-E(V)}{\mathrm{P} 2^{(m)}} \\
& =\frac{\operatorname{var}(V)+E^{2}(V)-E(V)}{\mathrm{P} 2^{(m)}} \\
& =\frac{m \rho(1-\rho)+(m \rho)^{2}-m \rho}{\mathrm{P} 2^{(m)}} \\
& =\frac{m(m-1) \rho^{2}}{\mathrm{P} 2^{(m)}}
\end{aligned}
$$

Therefore, the analogue of Kvam's Eq. C-6 is

$$
\frac{E[U(U-1)]}{(m-1) E(U)}=\frac{\rho}{1-(1-\rho)^{m-1}} \text {. }
$$

Estimate the expectations from the data, giving the equation 
$\frac{\sum_{k=2}^{m} k(k-1) n_{k}{ }^{(m)}}{(m-1) \sum_{k=2}^{m} k n_{k}^{(m)}}=\frac{\rho}{1-(1-\rho)^{m-1}}$

This must be solved numerically for the MM estimate of $\rho$.

Just as for the MLE, there are two special cases. First, if $m=2$, Eq. C-7 reduces to $1=\rho / \rho$, which does not determine a unique value of $\rho$. This is what must follow from the third bullet in the above background section. The second special case occurs when the data for some $m>2$ contain no multiple failures of more than two components, that is, $n_{k}^{(m)}=0$ for $k>2$. Then the left-hand side of the Eq. C-7 reduces to $1 /(m-1)$. The right-hand side equals this value only when $\rho=0$. This is mentioned in the fourth bullet in the background section.

This method gives a separate estimate of $\rho$ for each $m$. In Ref. [C-2], Kvam combines results from various $m$ without specifying the details. At present we do not see a "best" way to obtain one estimate from the combined data using all the different group sizes $m$.

\section{Impact Vectors or Data?}

The methods above all assume that the numbers of failed components are known. This is also assumed in Refs. [C-1] and [C-2]. However, the events occurring in failure reports are often not so clear. For example, it might happen that two pumps were clearly in a failed state but a third was degraded and might have failed if demanded. For this reason, impact vectors are defined, which are the expected numbers of events, in the probability sense of expected values. For details, see Ref. [C-3]. In the first example considered here, suppose that the analyst decides, with probability 0.9 , that the third pump was not failed, and with probability 0.1 the third pump was failed. Then the vector $\left(n_{1}{ }^{(3)}, n_{2}{ }^{(3)}, n_{3}{ }^{(3)}\right)=(0,1,0)$ under the first hypothesis and $=(0,0,1)$ under the second hypothesis. The impact vector is the expected value, or weighted average, of the two. This is denoted $\left(f_{1}{ }^{(3)}, f_{2}{ }^{(3)}, f_{3}{ }^{(3)}\right)=(0,0.9,0.1)$.

In practice, since the true numbers are not easily known, the probability-weighted averages, that is, the impact vectors, must be used. However, replacing the theoretically true numbers with the expected numbers does not interfere with finding $\rho$ to make the derivative in Eq. C-4 equal to zero or to solve Eq. C-7. This is how the MLE or MM estimate of $\rho$ can be found in practice.

\section{References}

[C-1] Mosleh A, Rasmuson DM, Marshall F. Guidelines on modeling common-cause failures in probabilistic risk assessment. NUREG/CR-5485, U.S. Nuclear Regulatory Commission, 1998.

[C-2] Kvam, P. Estimation Techniques for Common Cause Failure Data with Different System Sizes. Technometrics, vol. 38, no. 4, pp. 382-388, 1996.

[C-3] Wierman TE, Rasmuson DM, Mosleh A. Common-cause failure database and analysis system: event data collection, classification, and coding. NUREG/CR-6268, Rev. 1, U.S. Nuclear Regulatory Commission, 2007. 


\section{APPENDIX D}

\section{GENERAL MAPPING UP FORMULA}

\section{Summary}

Ref. [D-1] and its predecessor [D-2] propose a method for mapping data up, that is, for inferring a number of CCFs in a group of size $M$, based on an actual observed number in a smaller group of size $m$, with $m<M$. The proposed method is to imagine that the smaller group is embedded as a subgroup of the larger group. Then estimate the number of additional component failures that would be seen, assuming that the additional failures occur according to the BFR model.

Using the above approach, this appendix gives explicit reasons and an explicit general formula for the method of Ref. [D-1]. Though derived differently, the formula here agrees with method of Ref. [D-1], and with all the values tabulated there except for one apparent error.

\section{Notation and Background}

The BFR model asserts that common causes, "shocks," occur externally to the components and affect the entire common-cause group of components. When such an event occurs the components have independent outcomes, each failing with probability $\rho$ or succeeding with probability $1-\rho$. Therefore, when a shock occurs in a group of size $m$, the probability of $k$ failures is

$\operatorname{Pr}(k$ out of $m$ componentsfail $)=\left(\begin{array}{c}m \\ k\end{array}\right) \rho^{k}(1-\rho)^{m-k}$.

Let $n_{k}^{(m)}$ denote the number of events with $k$ failed components. If $N$ common-cause shocks occur, the value of $n_{k}^{(m)}$ is random,

$n_{k}^{(m)} \sim \operatorname{binomial}(N, \operatorname{Pr}(k$ out of $m$ components fail $))$,

and the expected number is

$E\left(n_{k}^{(m)}\right)=N * \operatorname{Pr}(k$ out of $m$ components fail $)$.

For the rest of this note, assume that the data consist of $n_{1}{ }^{(m)}, \ldots, n_{m}{ }^{(m)}$, the numbers of events observed in a group of size $m$. Assume that these counts are used to infer the corresponding counts in a larger group, of size $M$. The value of $\rho$ must be estimated somehow; this will not be dealt with here.

The method regards the smaller group of size $m$ as a subgroup of the larger group. More precisely, it behaves the same as a subgroup of the larger group. Then the BFR formulas are used to estimate the performance of the entire group, given the observed performance of the subgroup.

\section{Formulas for mapping up}

Ref. [D-1] presents its method by example, and this note does the same, with $m=2, M=4$. Table 1 shows the 16 possible sets of failed components. The components A and B are observed failed components in the group of 2 components. Because they are observable, they are shown in bold face. The components $\mathrm{C}$ and $\mathrm{D}$ are those that might fail if the 2-component system is embedded in a 4-component system. 
Table D-1. Example of mapping up from 2-component system to 4 component system.

\begin{tabular}{|c|c|c|c|c|c|c|}
\hline & \multicolumn{3}{|c|}{ Failed comps. } & Failures & Total & $\operatorname{Pr}(2 n d)$ \\
\hline 1 & $-\quad-$ & - & & 0 & 0 & $(1-\rho)^{2}$ \\
\hline 2 & A - & - & - & 1 & 1 & $(1-\rho)^{2}$ \\
\hline 3 & $-\quad \mathbf{B}$ & - & - & 1 & 1 & $(1-\rho)^{2}$ \\
\hline 4 & $-\quad-$ & $\mathrm{C}$ & - & 0 & 1 & $\rho(1-\rho)$ \\
\hline 5 & $-\quad-$ & - & $\mathrm{D}$ & 0 & 1 & $\rho(1-\rho)$ \\
\hline 6 & A $\mathbf{B}$ & - & - & 2 & 2 & $(1-\rho)^{2}$ \\
\hline 7 & A - & $\mathrm{C}$ & - & 1 & 2 & $\rho(1-\rho)$ \\
\hline 8 & A - & - & $\mathrm{D}$ & 1 & 2 & $\rho(1-\rho)$ \\
\hline 9 & $-\quad \mathbf{B}$ & $\mathrm{C}$ & - & 1 & 2 & $\rho(1-\rho)$ \\
\hline 10 & $-\quad \mathbf{B}$ & - & $\mathrm{D}$ & 1 & 2 & $\rho(1-\rho)$ \\
\hline 11 & - & $\mathrm{C}$ & $\mathrm{D}$ & 0 & 2 & $\rho^{2}$ \\
\hline 12 & $-\quad \mathbf{B}$ & $\mathrm{C}$ & $\mathrm{D}$ & 1 & 3 & $\rho^{2}$ \\
\hline 13 & A - & $\mathrm{C}$ & $\mathrm{D}$ & 1 & 3 & $\rho^{2}$ \\
\hline 14 & A $\mathbf{B}$ & - & D & 2 & 3 & $\rho(1-\rho)$ \\
\hline 15 & A $\mathbf{B}$ & $\mathrm{C}$ & - & 2 & 3 & $\rho(1-\rho)$ \\
\hline 16 & A $\mathbf{B}$ & $\mathrm{C}$ & D & 2 & 4 & $\rho^{2}$ \\
\hline
\end{tabular}

For example, row 2 has a failure of $\mathrm{A}$, but of no other components. Row 12 shows a failure of $\mathrm{B}, \mathrm{C}$, and D.

To demonstrate the formulas for mapping up, suppose first that $n_{1}{ }^{(2)}$ events have been observed in which A or B fails, but not both. Let us find the expected number of events with exactly 1 component failing out of the 4 in the larger group. This happens if neither $\mathrm{C}$ nor $\mathrm{D}$ fails in addition to $\mathrm{A}$ or $\mathrm{B}$. From rows 2 and 3 of the table, we see that the expected number of events with A or B and nothing else failing is

$(1-\rho)^{2} n_{1}^{(2)}$.

However, these are not the only cases with exactly 1 failed component out of 4 . Rows 4 and 5 also have this total number of failures, and by symmetry, all four of rows 2 through 5 have the same probability in the 4-component group. Therefore, the expected number of events with 1 failure out of 4 is $n_{1}^{(4)}=2(1-\rho)^{2} n_{1}^{(2)}$.

This agrees with Table C-5 of Ref. [D-1], although that table uses the notation $P$ instead of $n$.

It is easy to overlook rows 4 and 5, because they were not observable in the data for the 2-component group. However, they must be counted. Anyone who objects to counting them should recall that similar reasoning is used when mapping independent failures up; in that situation, the additional independent 
failures inferred for the larger group are all in events for which the smaller group of components were successful.

Let us now go on to consider $n_{2}{ }^{(4)}$. A total of two failed components can result when 0,1 , or 2 of A and B fail. Row 6 contributes to the total by an expected count of

$(1-\rho)^{2} n_{2}^{(2)}$.

Also, rows 7 through 10 contribute

$2 \rho(1-\rho)^{2} n_{1}^{(2)}$.

In this last expression, the multiplier of 2 results from considering either $\mathrm{C}$ or $\mathrm{D}$ as a failed component; $n_{1}{ }^{(2)}$ counts of all the events in which A or B fails, but not both.

Finally, we must deal with row 11, in which neither A nor B is observed to fail. There are 6 cases having a total of exactly 2 failed components, rows 6 through 11 . By symmetry, they all have the same probability. Therefore, the total for $n_{2}{ }^{(4)}$ must be $6 / 5$ of the total from the 5 rows considered above:

$n_{2}^{(4)}=(6 / 5)\left[(1-\rho)^{2} n_{2}^{(2)}+2 \rho(1-\rho)^{2} n_{1}^{(2)}\right]$.

This does not agree with Table C-5 of Ref. [D-1]. It is believed that the tabulated value is erroneous, as discussed at the end of this note.

The remaining cases are straightforward. For $n_{3}{ }^{(4)}$, rows 12 and 13 contribute

$\rho^{2} n_{1}^{(2)}$,

and rows 14 and 15 contribute

$2 \rho(1-\rho)^{2} n_{2}^{(2)}$.

This results in

$n_{3}^{(4)}=\rho^{2} n_{1}{ }^{(2)}+2 \rho(1-\rho)^{2} n_{2}{ }^{(2)}$.

This agrees with Table C-5 of Ref. [D-1].

Finally, row 16 gives

$N_{4}^{(4)}=\rho^{2} n_{2}{ }^{(2)}$,

agreeing with Table C-5 of Ref. [D-1],

Using reasoning as in the example, it can be shown that the general formula for mapping up from size $m$ to size $M$ is

$n_{K}{ }^{(M)}=\frac{\left(\begin{array}{c}M \\ K\end{array}\right)}{\left(\begin{array}{c}M \\ K\end{array}\right)-\left(\begin{array}{c}M-m \\ K\end{array}\right)}\left[\sum_{k}\left(\begin{array}{c}M-m \\ K-k\end{array}\right) \rho^{K-k}(1-\rho)^{(M-m)-(K-k)} n_{k}{ }^{(m)}\right]$.

In the summation here, the limits on $k$ are such that all the terms are defined and $k>0$, that is,

$\max [1, m-(M-K)] \leq k \leq \min (K, m)$.

The multiplying fraction outside the square brackets accounts for the cases with no failures observed in the $m$-component group.

$\left(\begin{array}{c}M \\ K\end{array}\right)$ is the number of ways to choose $K$ failed components out of $M$, and 
$\left(\begin{array}{c}M-m \\ K\end{array}\right)$ is the number of ways to choose $K$ of $M-m$, so that none of the first $m$ components fail.

Therefore, the numerator of the fraction is the number of equally probable ways for $K$ of $M$ components to fail, and the denominator is the number of those ways that can be assessed based on observed failures in the $m$-component group. The fraction is defined to be 1 if $K>M-m$.

Using direct but tedious algebra, one can show that Eq. D-1 is identical to that applied in the examples on p. C-12 of Ref. [D-1] and to generalization of those examples. It also agrees with the formulas in Table C-5 of Ref. [D-1], except for the apparently erroneous tabulated value for $n_{3}{ }^{(4)}$.

\section{References}

[D-1] Mosleh A, Rasmuson DM, Marshall F. Guidelines on modeling common-cause failures in probabilistic risk assessment. NUREG/CR-5485, U.S. Nuclear Regulatory Commission, 1998.

[D-2] Mosleh A, Fleming KN, Parry GW, Paula HM, Worledge DH, Rasmuson DM. 1989. Procedures for Treating Common Cause Failures in Safety and Reliability Studies: Analytical Background and Techniques. NUREG/CR-4720 Vol. 2, U.S. Nuclear Regulatory Commission; 1989. 